
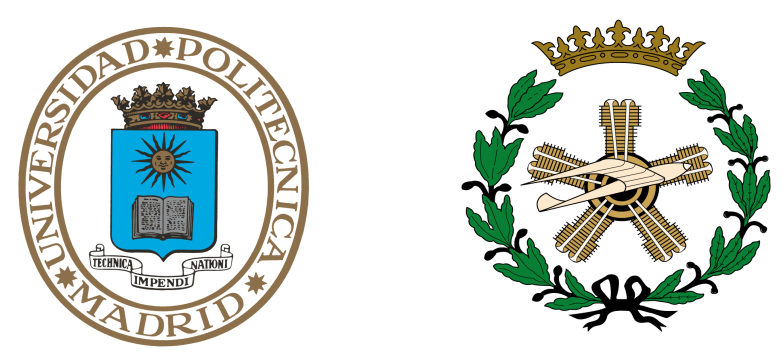

Universidad Politécnica de Madrid

Escuela Técnica Superior de Ingenieros Aeronáuticos

\title{
MÉTODO COMPUTACIONAL PARA LA ACELERACIÓN DE ANÁLISIS PARAMÉTRICOS DE MODIFICACIONES LOCALES EN ESTRUCTURAS COMPLEJAS SOMETIDAS
}

\section{A CARGAS DINÁMICAS}

\author{
Tesis Doctoral \\ Francisco José Herrada Martín \\ Ingeniero Aeronáutico
}



Departamento de Aeronaves y Vehículos Espaciales

Escuela Técnica Superior de Ingenieros Aeronáuticos

\section{Método computacional para la aceleración de análisis paramétricos de modificaciones locales en estructuras complejas sometidas cargas dinámicas}

Tesis Doctoral

Francisco José Herrada Martín

Ingeniero Aeronáutico

Directores de la Tesis Doctoral

Francisco Javier Montáns Leal

Dr. Ingeniero Industrial

Catedrático de Universidad
Iván Muñoz Díaz

Dr. Ingeniero Industrial

Profesor Titular 



\section{TRIBUNAL}

\section{Presidente:}

\section{Alberto Fraile de Lerma \\ Dr. Ingeniero Industrial}

Universidad Politécnica de Madrid

Vocal:

\section{Ricardo Atienza Pascual}

Dr. Ingeniero Aeronáutico

Universidad Alfonso X el Sabio

Vocal:

Dña. $\mathbf{M}^{a}$ del Carmen Serna Moreno

Dr. Ingeniero Industrial

Universidad de Castilla-La Mancha

Suplente:

D. Fernando Cabrerizo García

Dr. Ingeniero Aeronáutico

Universidad Alfonso X el Sabio
Vocal:

\section{Miguel Ángel Caminero Torija}

Dr. Ingeniero Industrial

Universidad de Castilla-La Mancha

Secretario:

\section{Marcos Latorre Ferrús}

Dr. Ingeniero Aeronáutico

Universidad Politécnica de Madrid

Suplente:

D. Juan José Benito Muñoz

Dr. Ingeniero Industrial

Universidad Nacional de Educación a

Distancia 

A mis padres, si hay algo bueno en mí se lo debo a ellos, y a Loreto, que me hace mejor cada día. 



\section{RESUMEN}

Dentro del análisis y diseño estructural surgen frecuentemente problemas de ingeniería donde se requiere el análisis dinámico de grandes modelos de elementos finitos que llegan a millones de grados de libertad y emplean volúmenes de datos de gran tamaño. La complejidad y dimensión de los análisis se dispara cuando se requiere realizar análisis paramétricos. Este problema se ha abordado tradicionalmente desde diversas perspectivas: en primer lugar, aumentando la capacidad tanto de cálculo como de memoria de los sistemas informáticos empleados en los análisis. En segundo lugar, se pueden simplificar los análisis paramétricos reduciendo su número o detalle y por último se puede recurrir a métodos complementarios a los elementos finitos para la reducción de sus variables y la simplificación de su ejecución manteniendo los resultados obtenidos próximos al comportamiento real de la estructura.

Se propone el empleo de un método de reducción que encaja en la tercera de las opciones y consiste en un análisis simplificado que proporciona una solución para la respuesta dinámica de una estructura en el subespacio modal complejo empleando un volumen de datos muy reducido. De este modo se pueden realizar análisis paramétricos variando múltiples parámetros, para obtener una solución muy aproximada al objetivo buscado. Se propone no solo la variación de propiedades locales de masa, rigidez y amortiguamiento sino la adición de grados de libertad a la estructura original para el cálculo de la respuesta tanto permanente como transitoria. Adicionalmente, su facilidad de implementación permite un control exhaustivo sobre las variables del problema y la implementación de mejoras como diferentes formas de obtención de los autovalores o la eliminación de las limitaciones de amortiguamiento en la estructura original.

El objetivo del método se puede considerar similar a los que se obtienen al aplicar el método de Guyan u otras técnicas de reducción de modelos empleados en dinámica estructural. Sin embargo, aunque el método permite ser empleado en conjunción con otros para obtener las ventajas de ambos, el presente procedimiento no realiza la condensación del sistema de ecuaciones, sino que emplea la información del sistema de ecuaciones completa estudiando tan solo la respuesta en las variables apropiadas de los puntos de interés para el analista. Dicho interés puede surgir de la necesidad de obtener la respuesta de las grandes estructuras en unos puntos determinados o de la necesidad de modificar la estructura en zonas determinadas para cambiar su comportamiento (respuesta en aceleraciones, velocidades o desplazamientos) ante 
cargas dinámicas. Por lo tanto, el procedimiento está particularmente indicado para la selección del valor óptimo de varios parámetros en grandes estructuras (del orden de cientos de miles de modos) como pueden ser la localización de elementos introducidos, rigideces, masas o valores de amortiguamientos viscosos en estudios previos en los que diversas soluciones son planteadas y optimizadas, y que en el caso de grandes estructuras, pueden conllevar un número de simulaciones extremadamente elevado para alcanzar la solución óptima.

Tras plantear las herramientas necesarias y desarrollar el procedimiento, se propone un caso de estudio para su aplicación al modelo de elementos finitos del UAV MILANO desarrollado por el Instituto Nacional de Técnica Aeroespacial. A dicha estructura se le imponen ciertos requisitos al incorporar un equipo en aceleraciones en punta de ala izquierda y desplazamientos en punta de ala derecha en presencia de la sustentación producida por una ráfaga continua de viento de forma sinusoidal. La modificación propuesta consiste en la adición de un equipo en la punta de ala izquierda, bien mediante un anclaje rígido, bien unido mediante un sistema de reducción de la respuesta dinámica con propiedades de masa, rigidez y amortiguamiento variables.

El estudio de los resultados obtenidos permite determinar la optimización de los parámetros del sistema de atenuación por medio de múltiples análisis dinámicos de forma que se cumplan de la mejor forma posible los requisitos impuestos con la modificación. Se comparan los resultados con los obtenidos mediante el uso de un programa comercial de análisis por el método de los elementos finitos lográndose soluciones muy aproximadas entre el modelo completo y el reducido.

La influencia de diversos factores como son el amortiguamiento modal de la estructura original, el número de modos retenidos en la truncatura o la precisión proporcionada por el barrido en frecuencia se analiza en detalle para, por último, señalar la eficiencia en términos de tiempo y volumen de datos de computación que ofrece el método propuesto en comparación con otras aproximaciones.

Por lo tanto, puede concluirse que el método propuesto se considera una opción útil y eficiente para el análisis paramétrico de modificaciones locales en grandes estructuras. 


\section{ABSTRACT}

When developing structural design and analysis some projects require dynamic analysis of large finite element models with millions of degrees of freedom which use large size data files. The analysis complexity and size grow if a parametric analysis is required. This problem has been approached traditionally in several ways: one way is increasing the power and the storage capacity of computer systems involved in the analysis. Other obvious way is reducing the total amount of analyses and their details. Finally, complementary methods to finite element analysis can also be employed in order to limit the number of variables and to reduce the execution time keeping the results as close as possible to the actual behaviour of the structure.

Following this third option, we propose a model reduction method that is based in a simplified analysis that supplies a solution for the dynamic response of the structure in the complex modal space using few data. Thereby, parametric analysis can be done varying multiple parameters so as to obtain a solution which complies with the desired objetive. We propose not only mass, stiffness and damping variations, but also addition of degrees of freedom to the original structure in order to calculate the transient and steady-state response. Additionally, the simple implementation of the procedure allows an in-depth control of the problem variables. Furthermore, improvements such as different ways to obtain eigenvectors or to remove damping limitations of the original structure are also possible.

The purpose of the procedure is similar to that of using the Guyan or similar model order reduction techniques. However, in our method we do not perform a true model order reduction in the traditional sense. Furthermore, additional gains, which we do not explore herein, can be obtained through the combination of this method with traditional model-order reduction procedures. In our procedure we use the information of the whole system of equations is used but only those nodes of interest to the analyst are processed. That interest comes from the need to obtain the response of the structure at specific locations or from the need to modify the structure at some suitable positions in order to change its behaviour (acceleration, velocity or displacement response) under dynamic loads. Therefore, the procedure is particularly suitable for parametric optimization in large structures with $>100000$ normal modes such as position of new elements, stiffness, mass and viscous dampings in previous studies where different solutions are devised and optimized, and in the case of large structures, can carry an extremely high 
number of simulations to get the optimum solution.

After the introduction of the required tools and the development of the procedure, a study case is proposed with use the finite element model (FEM) of the MILANO UAV developed by Instituto Nacional de Técnica Aeroespacial. Due to an equipment addition, certain acceleration and displacement requirements on left wing tip and right wing tip, respectively, are imposed. The structure is under a continuous sinusoidal wind gust which produces lift. The proposed modification consists of the addition of an equipment in left wing tip clamped through a rigid attachment or through a dynamic response reduction system with variable properties of mass, stiffness and damping.

The analysis of the obtained results allows us to determine the optimized parametric by means of multiple dynamic analyses in a way such that the imposed requirements have been accomplished in the best possible way. The results achieved are compared with results from a commercial finite element analysis software, showing a good correlation.

Influence of several factors such as the modal damping of the original structure, the number of modes kept in the modal truncation or the precission given by the frequency sweep is analyzed. Finally, the efficiency of the proposed method is addressed in tems of computational time and data size compared with other approaches.

From the analyses performed, we can conclude that the proposed method is a useful and efficient option to perform parametric analysis of possible local modifications in large structures. 


\section{Agradecimientos}

Es difícil expresar en palabras los sentimientos. Cuando comencé hace años los estudios preliminares de esta tesis, consulté a mis compañeros del INTA y nos embarcamos juntos en esta aventura guiados por Jesús López Díez. A Jesús le perdimos (le mando un abrazo enorme a su familia y amigos), cambiaron las circunstancias, en particular mi trabajo, pero tres de los que iniciamos la andadura hemos seguido juntos hasta cumplir este último hito.

Tengo que agradecer a mis amigos y compañeros del Área de Estructuras y Mecanismos del INTA su ayuda y colaboración tanto técnica como personal desde el principio. De ellos siempre he recibido un sí por respuesta. En los últimos tiempos he podido contar con el apoyo de mis compañeros de mi actual trabajo, así como con la ayuda y colaboración de los miembros del Departamento de Aeronaves y Vehículos Espaciales, que han estado siempre atentos a cualquier detalle para ayudarme.

No todo ha sido fácil. Hace dos años, en el momento más delicado, apareció la figura fundamental de Francisco (Paco) Montáns. Él junto a Iván Muñoz me han enseñado la pasión por la Investigación y la Ciencia y me han regalado, no solo su sabiduría y conocimientos, sino su cercanía y acogida que agradeceré siempre.

Clases, cursos, trabajos, programas, ensayos, estudio... ha habido muchos momentos buenos y otros duros en estos años. Durante muchas horas, gran parte de ellas robadas al sueño, los tres antiguos compañeros del INTA hemos compartido trabajo e ilusiones, vivido los momentos más importantes de nuestras vidas y espero que llegue pronto una nueva aventura para embarcarme de nuevo con ellos. Por lo tanto, al Doctor Ingeniero Aeronáutico Iñaki Armendáriz y, especialmente, a Juan García mi agradecimiento más sincero.

Finalmente, las palabras se quedan cortas para expresar lo que le debo a mi familia: de ellos es también esta tesis.

Sin todos jamás estaría escribiendo estas palabras. 


\section{Índice General}

1 INTRODUCCIÓN 1

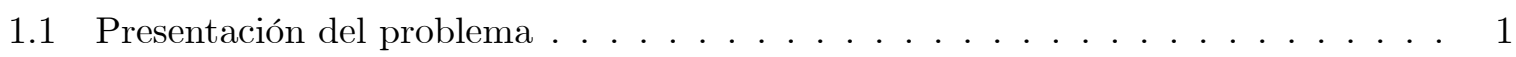

1.2 Parametrización y diseño estructural . . . . . . . . . . . . . . . . 2

1.3 Modificación estructural . . . . . . . . . . . . . . . . . . 3

1.4 Reducción de modelos . . . . . . . . . . . . . . . . . . . . . . . . 4

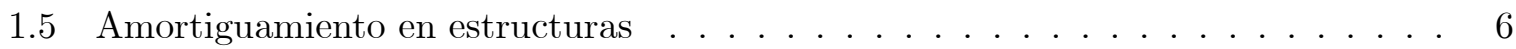

1.6 Atenuación de excitaciones dinámicas . . . . . . . . . . . . . . . . . 8

1.7 Objetivos y alcance de la tesis . . . . . . . . . . . . . . 12

1.8 Estructura de la tesis . . . . . . . . . . . . . . . . . . . . . . 13

2 DESCRIPCIÓN DEL MÉTODO DE ACELERACIÓN DE ANÁLISIS DINÁMI$\begin{array}{ll}\operatorname{COS} & 15\end{array}$

2.1 Descomposición modal . . . . . . . . . . . . . . . . . . . . 15

2.2 Introducción de amortiguamiento adicional no proporcional. . . . . . . . . . . 18

2.3 Adición de elementos de masa y rigidez. . . . . . . . . . . . . . . . 20

2.4 Adición de grados de libertad . . . . . . . . . . . . . . . . . . . 21

2.5 Solución del problema general . . . . . . . . . . . . . . . . . 22

3 DESCRIPCIÓN DEL CASO DE ANÁLISIS 29

3.1 Descripción del modelo . . . . . . . . . . . . . . . . . . . . . . . . . . . . 29

3.2 Modelo del ala . . . . . . . . . . . . . . . . . . . . . . 31

3.3 Materiales y propiedades . . . . . . . . . . . . . . . . 33

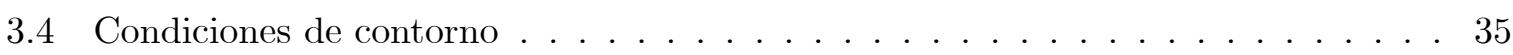


3.5 Uniones . . . . . . . . . . . . . . . . . . . . . . . . 39

3.6 Descripción del caso de carga . . . . . . . . . . . . . . . . . . . . . . 40

3.7 Procedimiento de análisis . . . . . . . . . . . . . . . . . . . 42

3.8 Descripción del tipo de análisis f . . . . . . . . . . . . . . . . . 43

3.9 Comportamiento modal . . . . . . . . . . . . . . . . . . 48

4 RESULTADOS DEL ANÁLISIS PARAMÉTRICO 51

4.1 Modificación del comportamiento modal . . . . . . . . . . . . . . . . . . . 62

4.2 Comparación con los resultados del modelo de elementos finitos . . . . . . . . . 66

4.3 Influencia del amortiguamiento modal de la estructura . . . . . . . . . . . . . 73

4.4 Influencia del número de modos considerados . . . . . . . . . . . . . . . . 92

4.5 Precisión por el barrido en frecuencia . . . . . . . . . . . . . . . . . . 112

5 CONCLUSIONES $\quad 125$

5.1 Discusión sobre los resultados . . . . . . . . . . . . . . . . . . 125

5.2 Conclusiones generales . . . . . . . . . . . . . . . . . . 132

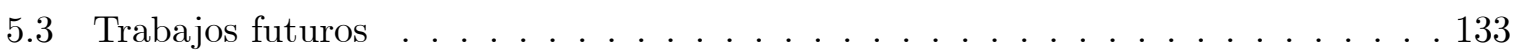




\section{Índice de figuras}

3-1 Prototipo MILANO (cortesía INTA) $\ldots \ldots \ldots \ldots$. . . . . . . . . . . 30

3-2 Prototipo ala MILANO $($ cortesía INTA $) \ldots \ldots \ldots \ldots \ldots$

3-3 Modelo FEM semiala MILANO (cortesía INTA) . . . . . . . . . . . . . . 32

3-4 FEM completo del ala (cortesía INTA) . . . . . . . . . . . . . . 33

3-5 Esquema de las capas de los largueros del ala exterior (cortesía INTA) . . . . . . 33

3-6 Esquema de las propiedades del modelo (cortesía INTA) . . . . . . . . . . . . . 34

3-7 Esquema de las capas de los largueros del ala central (cortesía INTA) . . . . . . 34

3-8 Esquema ensamblaje de las costillas (cortesía INTA) . . . . . . . . . . . . . 35

3-9 Herraje ala central delantero/trasero (cortesía INTA) . . . . . . . . . . . 36

3-10 Herraje ala exterior delantero/trasero $($ cortesía INTA $) \ldots \ldots$. . . . . . . 36

3-11 Herraje ala-fuselaje trasero $($ cortesía INTA $) \ldots \ldots$. . . . . . . . . 37

3-12 Herraje ala-fuselaje delantero $($ cortesía INTA $) \ldots \ldots$. . . . . . . . . 37

3-13 Condiciones de contorno del modelo FEM (cortesía INTA) . . . . . . . . . . . 38

3-14 Condición contorno herraje delantero (cortesía INTA) $\ldots \ldots \ldots$. . . . . . 38

3-15 Condición contorno herraje trasero $($ cortesía INTA) . . . . . . . . . . . . 39

3-16 Unión ala central-ala exterior $($ cortesía INTA) . . . . . . . . . . . . . . . . 40

3-17 Estaciones control en semiala izquierda para análisis por adición equipo en punta ala $($ cortesía INTA $) \ldots \ldots \ldots \ldots$. . . . . . . . . . . . . . . . . . 44

3-18 Esquema de análisis paramétrico de adición de equipo en punta de ala . . . . . . 44

3-19 Modo 1 de la estructura original: $7.6 \mathrm{~Hz} \ldots \ldots$. . . . . . . . . . . . . . . . . 49

3-20 Modo 2 de la estructura original: $9.1 \mathrm{~Hz} \ldots \ldots \ldots \ldots$. . . . . . . . . . . . . 49

3-21 Modo 3 de la estructura original: $15.7 \mathrm{~Hz} \ldots \ldots \ldots$. . . . . . . . . . . 50 
4-1 Análisis paramétrico. Resumen de respuestas en aceleraciones en punta de ala izquierda . . . . . . . . . . . . . . . . . . 52

4-2 Respuesta en frecuencia de aceleraciones en punta de ala izquierda . . . . . . . . 53

4-3 Respuesta en frecuencia de aceleraciones en inicio estrechamiento de semiala

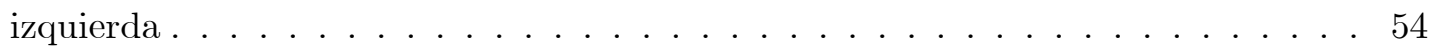

4-4 Respuesta en frecuencia de aceleraciones en encastre de semiala izquierda . . . . 54

4-5 Análisis paramétrico en masa . . . . . . . . . . . . . . . 55

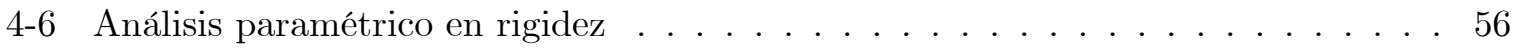

4-7 Análisis paramétrico en amortiguamiento . . . . . . . . . . . . . 57

4-8 Superficie paramétrica rigidez-amortiguamiento $\ldots \ldots \ldots \ldots \ldots$

4-9 Gráfica de líneas isoparamétricas k-c . . . . . . . . . . . . . . . . 59

4-10 Respuesta en frecuencia de desplazamiento en punta de ala derecha . . . . . . . 60

4-11 Respuesta en frecuencia de desplazamiento en encastre de semiala izquierda . . . 61

4-12 Respuesta en frecuencia de desplazamiento en sección inicio estrechamiento de semiala izquierda . . . . . . . . . . . . . . . . . . 6 61

4-13 Respuesta en frecuencia de desplazamiento de punta de ala izquierda . . . . . . . 62

4-14 Modo 1 de la estructura modificada: $6.0 \mathrm{~Hz} \ldots \ldots$. . . . . . . . . . . . . . 63

4-15 Modo 2 de la estructura modificada: $7.9 \mathrm{~Hz} \ldots \ldots \ldots$. . . . . . . . . . 63

4-16 Modo 3 de la estructura modificada: $8.2 \mathrm{~Hz} \ldots \ldots \ldots$. . . . . . . . . . . . 64

4-17 Modo 4 de la estructura modificada: $8.3 \mathrm{~Hz}$. . . . . . . . . . . . . . . . . . . 64

4-18 Modo 5 de la estructura modificada: $11.2 \mathrm{~Hz} \ldots \ldots \ldots$. . . . . . . . . . 65

4-19 Modo 6 de la estructura modificada: $16.1 \mathrm{~Hz} \ldots \ldots \ldots$. . . . . . . . . 65

4-20 Respuesta en frecuencia de aceleraciones en punta de ala izquierda (rango frecuencia $0.5 \mathrm{~Hz}-30 \mathrm{~Hz}) \ldots \ldots \ldots \ldots \ldots \ldots \ldots$

4-21 Respuesta en frecuencia de aceleraciones en punta de ala del modelo FEM . . . . 67

4-22 Respuesta temporal de aceleraciones en punta de ala izquierda a frecuencia $6.1 \mathrm{~Hz} 69$

4-23 Respuesta temporal de aceleraciones en punta de ala izquierda con frecuencia 7.6 $\mathrm{Hz} \ldots \ldots \ldots \ldots \ldots \ldots \ldots \ldots$

4-24 Respuesta temporal de aceleraciones en punta de ala izquierda a frecuencia $8.4 \mathrm{~Hz} 70$ 
4-25 Respuesta temporal de aceleraciones en punta de ala izquierda a frecuencia 6.1

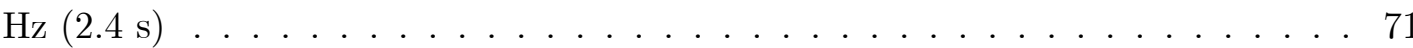

4-26 Respuesta temporal de aceleraciones en punta de ala izquierda a frecuencia 7.6

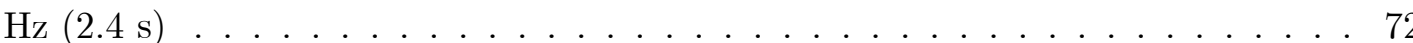

4-27 Respuesta temporal de aceleraciones en punta de ala izquierda a frecuencia 8.4

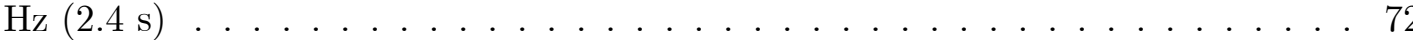

4-28 Análisis paramétrico. Resumen de respuestas en aceleraciones en punta de ala izquierda (amortiguamiento modal $5 \%) \ldots \ldots \ldots \ldots$

4-29 Respuesta en frecuencia de aceleraciones en punta de ala izquierda (amortiguamiento modal $5 \%) \ldots \ldots \ldots \ldots \ldots \ldots \ldots \ldots$

4-30 Respuesta en frecuencia de aceleraciones en inicio de estrechamiento de semiala izquierda (amortiguamiento modal $5 \%) \ldots \ldots \ldots \ldots$. . . . . . . . 75

4-31 Respuesta en frecuencia de aceleraciones en encastre de semiala izquierda (amortiguamiento modal $5 \%) \ldots \ldots \ldots \ldots \ldots \ldots$

4-32 Análisis paramétrico en masa (amortiguamiento modal 5\%) . . . . . . . . . . 76

4-33 Análisis paramétrico en rigidez (amortiguamiento modal 5\%) . . . . . . . . . 77

4-34 Análisis paramétrico en amortiguamiento (amortiguamiento modal 5\%) . . . . 77

4-35 Gráfica de líneas isoparamétricas k-c (amortiguamiento modal 5\%) . . . . . . . 78

4-36 Respuesta en frecuencia de aceleración en punta de ala izquierda del modelo FEM (amortiguamiento modal $5 \%) \ldots \ldots \ldots$. . . . . . . . . . 80

4-37 Respuesta temporal de aceleraciones en punta de ala izquierda a frecuencia 6.2 $\mathrm{Hz}$ (amortiguamiento modal $5 \%) \ldots \ldots \ldots \ldots$. . . . . . . . . . 80

4-38 Respuesta temporal de aceleraciones en punta de ala izquierda a frecuencia 7.6 $\mathrm{Hz}$ (amortiguamiento modal $5 \%) \ldots \ldots \ldots \ldots$. . . . . . . . . . . 81

4-39 Respuesta temporal de aceleraciones en punta de ala izquierda a frecuencia 8.7 $\mathrm{Hz}($ amortiguamiento modal $5 \%) \ldots \ldots \ldots$. . . . . . . . . . . 81

4-40 Análisis paramétrico. Resumen de respuestas en aceleraciones en punta de ala izquierda (amortiguamiento modal nulo) . . . . . . . . . . . . . . . . . 83

4-41 Respuesta en frecuencia de aceleraciones en punta de semiala izquierda (amortiguamiento modal nulo $\ldots \ldots \ldots$. . . . . . . . . . . . . . . . . . 84 
4-42 Respuesta en frecuencia de aceleraciones en inicio de estrechamiento de semiala izquierda (amortiguamiento modal nulo) . . . . . . . . . . . . . . . . . 85

4-43 Respuesta en frecuencia de aceleraciones en encastre de semiala izquierda (amortiguamiento modal nulo $\ldots \ldots \ldots \ldots \ldots$. . . . . . . . . . . . 85

4-44 Análisis paramétrico en masa (amortiguamiento modal nulo) . . . . . . . . . . 86

4-45 Análisis paramétrico en rigidez (amortiguamiento modal nulo) . . . . . . . . . . 86

4-46 Análisis paramétrico en amortiguamiento (amortiguamiento modal nulo) . . . . . 87

4-47 Gráfica de líneas isoparamétricas k-c (amortiguamiento modal nulo) . . . . . . 87

4-48 Respuesta temporal de aceleraciones en punta de ala izquierda a frecuencia 6.1 $\mathrm{Hz}$ para amortiguamiento modal $0 \% \ldots \ldots \ldots$

4-49 Respuesta temporal de aceleraciones en punta de ala izquierda a frecuencia 7.6 $\mathrm{Hz}$ para amortiguamiento modal $0 \% \ldots \ldots \ldots$

4-50 Respuesta temporal de aceleraciones en punta de ala izquierda a frecuencia 8.4 $\mathrm{Hz}$ para amortiguamiento modal $0 \% \ldots \ldots \ldots$. . . . . . . . . . 90

4-51 Análisis paramétrico en amortiguamiento modal . . . . . . . . . . . . . . . . 92

4-52 Análisis paramétrico. Resumen de respuesta en frecuencia de aceleraciones en

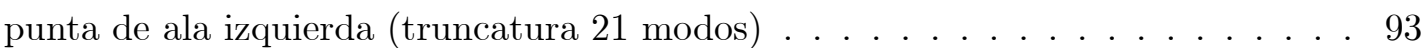

4-53 Respuesta en frecuencia de aceleraciones en punta de ala izquierda (truncatura 21 modos $\ldots \ldots \ldots \ldots \ldots \ldots \ldots$

4-54 Respuesta en frecuencia de aceleraciones en inicio de estrechamiento en semiala izquierda (truncatura 21 modos $) \ldots \ldots \ldots \ldots$. . . . . . . . . . . 94

4-55 Respuesta en frecuencia de aceleraciones en encastre de semiala izquierda (truncatura 21 modos $) \ldots \ldots \ldots \ldots \ldots$. . . . . . . . . . . . . . . 94

4-56 Análisis paramétrico en masa (truncatura 21 modos) . . . . . . . . . . . . . 95

4-57 Análisis paramétrico en rigidez (truncatura 21 modos) . . . . . . . . . . . . . . 96

4-58 Análisis paramétrico en amortiguamiento (truncatura 21 modos) . . . . . . . . . 96

4-59 Gráfica de líneas isoparamétricas k-c (truncatura 21 modos) . . . . . . . . . . . . 97

4-60 Análisis paramétrico. Resumen de respuesta en frecuencia de aceleraciones en punta de ala izquierda $($ truncatura 60 modos $) \ldots \ldots$. . . . . . . . . . 98 
4-61 Respuesta en frecuencia de aceleraciones en punta de ala izquierda (truncatura 60 modos $\ldots \ldots \ldots \ldots \ldots \ldots \ldots \ldots \ldots$

4-62 Respuesta en frecuencia de aceleraciones en inicio de estrechamiento en semiala izquierda (truncatura 60 modos $) \ldots \ldots \ldots \ldots \ldots$

4-63 Respuesta en frecuencia de aceleraciones en encastre de semiala izquierda (trun-

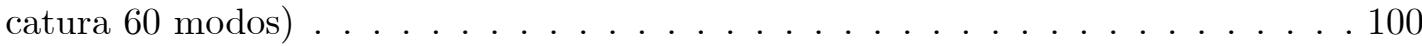

4-64 Análisis paramétrico en masa (truncatura 60 modos) . . . . . . . . . . . . . . . 101

4-65 Análisis paramétrico en rigidez (truncatura 60 modos) . . . . . . . . . . . . . . . 102

4-66 Análisis paramétrico en amortiguamiento (truncatura 60 modos) . . . . . . . . . 102

4-67 Gráfica de líneas isoparamétricas k-c (truncatura 60 modos) . . . . . . . . . . . . 103

4-68 Análisis paramétrico. Resumen de respuesta en frecuencia de aceleraciones en

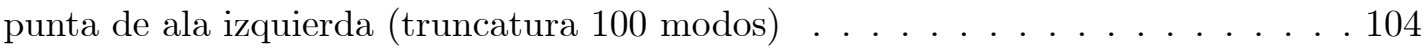

4-69 Respuesta en frecuencia de aceleraciones en punta de ala izquierda (truncatura

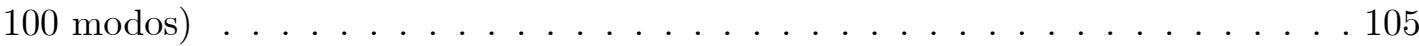

4-70 Respuesta en frecuencia de aceleraciones en inicio de estrechamiento en semiala izquierda (truncatura 100 modos) . . . . . . . . . . . . . . . . . . 106

4-71 Respuesta en frecuencia de aceleraciones en encastre de semiala izquierda (trun-

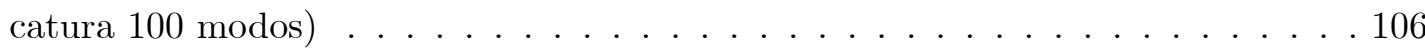

4-72 Análisis paramétrico en masa (truncatura 100 modos) . . . . . . . . . . . . . 107

4-73 Análisis paramétrico en rigidez (truncatura 100 modos) . . . . . . . . . . . . 108

4-74 Análisis paramétrico en amortiguamiento (truncatura 100 modos) . . . . . . . . . 108

4-75 Gráfica de líneas isoparamétricas k-c (truncatura 100 modos) . . . . . . . . . . . 109

4-76 Análisis paramétrico. Resumen de respuesta en frecuencia de aceleraciones en punta de ala izquierda (barrido 55 frecuencias) . . . . . . . . . . . . . . . . 112

4-77 Respuesta en frecuencia de aceleraciones en punta de ala izquierda (barrido 55

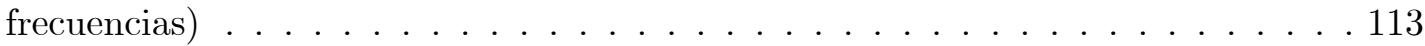

4-78 Respuesta en frecuencia de aceleraciones en inicio de estrechamiento en semiala izquierda (barrido 55 frecuencias) . . . . . . . . . . . . . . . . . . . . . 114

4-79 Respuesta en frecuencia de aceleraciones en encastre de semiala izquierda (barrido 55 frecuencias $)$. . . . . . . . . . . . . . . . . . . . . . . 115 
4-80 Análisis paramétrico en masa (barrido 55 frecuencias) . . . . . . . . . . . . 115

4-81 Análisis paramétrico en rigidez (barrido 55 frecuencias) $\ldots \ldots \ldots$. . . . . 116

4-82 Análisis paramétrico en amortiguamiento (barrido 55 frecuencias) . . . . . . . 116

4-83 Gráfica de líneas isoparamétricas k-c (barrido 55 frecuencias) . . . . . . . . . . . 117

4-84 Análisis paramétrico. Resumen de respuesta en frecuencia de aceleraciones en punta de ala (barrido de frecuencias incrementado) . . . . . . . . . . . . . 118

4-85 Respuesta en frecuencia de aceleraciones en punta de ala izquierda (barrido de frecuencias incrementado) . . . . . . . . . . . . . . . . . . . . . 119

4-86 Respuesta en frecuencia de aceleraciones en inicio de estrechamiento de semiala izquierda (barrido de frecuencias incrementado) . . . . . . . . . . . . . . . . 120

4-87 Respuesta en frecuencia de aceleración en encastre de semiala izquierda (barrido de frecuencias incrementado) . . . . . . . . . . . . . . . . . 120

4-88 Análisis paramétrico en masa (barrido de frecuencias incrementado) . . . . . . . 121

4-89 Análisis paramétrico en rigidez (barrido de frecuencias incrementado) . . . . . . 121

4-90 Análisis paramétrico en amortiguamiento (barrido de frecuencias incrementado) . 122

4-91 Gráfica de líneas isoparamétricas k- c (barrido de frecuencias incrementado) . . . 122 


\section{Índice de tablas}

4.1 Comparación de máximos de aceleraciones de los diferentes análisis sin TMD . 67

4.2 Comparación de máximos de aceleraciones de los diferentes análisis con TMD . . 68

4.3 Comparación de máximos de aceleraciones con análisis transitorio . . . . . . . . 70

4.4 Comparación de máximos de aceleraciones con análisis transitorio ampliado . . . 71

4.5 Comparación de análisis sin TMD con amortiguamiento incrementado . . . . . . 78

4.6 Comparación de análisis con TMD con amortiguamiento incrementado . . . . . . 79

4.7 Comparación de máximos de aceleraciones con análisis transitorio con amortiguamiento incrementado . . . . . . . . . . . . . . . 82

4.8 Comparación de análisis sin TMD con amortiguamiento nulo . . . . . . . . . 88

4.9 Comparación de análisis con TMD con amortiguamiento nulo . . . . . . . . . . 88

4.10 Comparación de máximos de aceleraciones con análisis transitorio con amortiguamiento nulo . . . . . . . . . . . . . . . . . . . . . . 91

4.11 Comparación de máximos de aceleraciones con variación modos considerados sin TMD . . . . . . . . . . . . . . . . . . . . . . . 110

4.12 Comparación de máximos de aceleraciones con variación modos considerados con TMD . . . . . . . . . . . . . . . . . . . . . . . 111

4.13 Comparación de máximos de aceleraciones con análisis transitorio con variación modos considerados . . . . . . . . . . . . . . . . . . . . . 111

4.14 Comparación de máximos de aceleraciones con variación barrido frecuencias sin

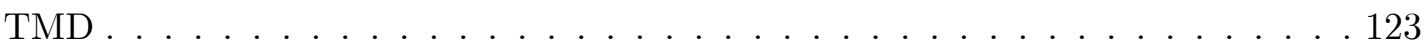

4.15 Comparación de máximos de aceleraciones con variación barrido frecuencias con TMD . . . . . . . . . . . . . . . . . . . . . . . . . . . . 124 
4.16 Comparación de máximos de aceleraciones con análisis transitorio con variación barrido frecuencias . . . . . . . . . . . . . . . . . . . . . . . . 124

5.1 Tiempos de computación de análisis en frecuencia . . . . . . . . . . . . . 130

5.2 Tiempos de computación del caso de análisis de referencia . . . . . . . . . . . . 131 


\section{Capítulo 1}

\section{INTRODUCCIÓN}

\subsection{Presentación del problema}

Una de las herramientas más destacadas para el diseño o análisis de estructuras es la simulación numérica. Esta técnica, apoyada por los exponenciales avances en la potencia de computación disponible, es cada vez más utilizada y se considera actualmente imprescindible en la mayoría de los proyectos. La lógica evolución de dicha herramienta, representada en el ámbito de aplicación por el Método de los Elementos Finitos, tiende a mejorar las simulaciones completando los modelos, tanto sus detalles y características como sus dimensiones [Mourelatos et al., 2012]. De esta forma, los modelos actualmente manejados en ingeniería son mucho más detallados y complejos que los de hace unos años [Garatani, 1999].

Sin embargo, o quizás por eso mismo, el manejo de grandes modelos sigue siendo aún un límite que se tiene presente en análisis pero especialmente en diseño de estructuras. Los modelos de millones de grados de libertad y volúmenes de datos de gigabytes requieren un tratamiento específico que puede implicar costes elevados debidos a diversas causas [Chen et al. , 2011]. En primer lugar porque la solución por defecto es escalar la potencia de cálculo y de almacenamiento de los sistemas informáticos empleados. El incremento de tiempo empleado en los análisis también impacta en el coste debido al uso de las licencias de los programas de análisis así como del consumo eléctrico y de refrigeración de las máquinas. La simplificación del propio modelo o del análisis en la mayoría de los casos no es satisfactoria ya que sufre limitaciones diversas en función de los métodos empleados. 
Por último, se puede recurrir a métodos para la reducción de sus variables y la simplificación de su ejecución manteniendo los resultados obtenidos próximos al comportamiento real de la estructura [Qu, 2004]. Estos métodos en algunos casos son complementarios con los elementos finitos e incluso están implementados en códigos comerciales [MSC.Software, 2012] son ampliamente utilizados para cierto tipo de problema. Esta forma de acometer el problema será tratado en un apartado posterior.

\subsection{Parametrización y diseño estructural}

El problema de la dimensión de los grandes modelos estructurales se complica cuando se trata de realizar análisis paramétricos, que integran gran número de análisis semejantes.

Este es un ámbito tecnológico en el que diseño y análisis se encuentran [Laan \& Tooren, 2004] y que ha sido explotado eficazmente en el ámbito aeronáutico, de forma que se tiende a la integración del diseño paramétrico, ya plenamente incorporado en las empresas, con el necesario análisis estructural generalmente realizado por el método de los elementos finitos [Tooren et al. , 2003].

Según [Rey, 2013] se puede decir que el diseño paramétrico es un diseño asociativo basado en parámetros que permite realizar modificaciones en la geometría del modelo de forma rápida y sencilla al estar las variables relacionadas entre sí, por lo que cualquier cambio en una variable se arrastra automáticamente al resto de ellas. Es decir, se puede ver como una técnica para el control y la manipulación de objetos de diseño a cualquier escala, que emplea algoritmos como métodos de generación de formas y posibilita la producción de formas complejas basadas en reglas sencillas para el control de cada uno de sus componentes. En la práctica, esto supone que se conoce cada elemento del diseño y sus formas derivadas por su expresión geométrica o matemática por lo que están totalmente determinados.

De forma análoga, se puede entender el análisis paramétrico como el conjunto de análisis gobernados por variables relacionadas entre sí. Es claro que en análisis estructural tanto el cambio del diseño geométrico como otros cambios como el de propiedades físicas [Cuschieri, 1990] tienen influencia en los resultados por lo que pueden usarse como posibles parametrizaciones. La selección de parámetros depende del tipo de análisis a ejecutar y pueden enlazarse si fuera 
procedente con las características adecuadas del diseño como se ha comentado. Por ejemplo en análisis dinámicos de rotores puede considerarse el rozamiento con el eje como parámetro [Xie et al. , 2015] y en casos más complejos como el desarrollado en [Armendariz et al. , 2015] para el caso de la pérdida de una pala de un turbohélice se realizan parametrizaciones en posición angular de la pala, velocidad angular, tamaño de pala, rigidez y amortiguamiento de la estructura.

Además de para la etapa de diseño, este tipo de análisis es una herramienta muy útil también para estudiar los efectos de eventuales modificaciones estructurales ya que permite ofrecer, no solo la identificación de una solución que cumpla los requisitos sino en algunos casos la optimización de un conjunto de variables [Wallack et al. , 1989].

Por lo tanto, la posibilidad de conocer la influencia de algunos parámetros como la rigidez o la masa de algún elemento estructural en la respuesta dinámica del sistema proporciona gran eficiencia a los análisis paramétricos.

\subsection{Modificación estructural}

Un claro ejemplo de uso de la parametrización es el de la modificación estructural. En el ámbito del análisis dinámico, consiste en la técnica por la que se modifican las propiedades físicas de una estructura para alterar su comportamiento dinámico [Nad, 2007]. Las características dinámicas de una estructura, frecuencias y modos propios, vienen determinadas por sus distribuciones de masa, rigidez y amortiguamiento. Los modelos de elementos finitos recogen dichas distribuciones de la estructura real simulando adecuadamente su comportamiento. Desde el punto de vista dinámico, salvo casos excepcionales, los cambios en las propiedades físicas de una estructura producen un cambio en su comportamiento dinámico, es decir en sus propiedades modales.

Las modificaciones estructurales suelen proponerse para la mejora de la respuesta de una estructura existente. Otro posible motivo es el diseño de una estructura que, estando sometida a un entorno de cargas dinámicas, debe cumplir unos requisitos ya sean de aceleraciones, velocidades o desplazamientos.

La determinación de la solución, ya sea del problema directo de conocer la alteración de la respuesta causada por la modificación estructural, o del problema inverso en el que se conoce 
inicialmente el comportamiento dinámico deseado, puede requerir conocer la relación entre las variables que intervienen en el problema [Aryana \& Bahai, 2003] por lo que la parametrización puede ser esencial para obtener la solución, o al menos, hacerlo eficientemente. Las modificaciones estructurales se han ido tratando de incorporar de diversas maneras al diseño estructural de forma que sus análisis sean precisos y adecuados tanto en tiempo como en necesidad de cálculo [Avitable, 2003].

\subsection{Reducción de modelos}

Como se ha explicado anteriormente, una de las formas de enfrentarse al manejo de grandes modelos estructurales es la reducción del modelo de forma que se reduzcan los costes tanto de tiempo como los que implicaría realizar análisis paramétricos de la estructura completa. Desde hace tiempo se han empleado numerosas aproximaciones al problema en diversos ámbitos científicos [Nabag et al. , 2010].

En este trabajo y como es habitual en dinámica estructural [MSC.Sofware, 2012], se comenzará el análisis dinámico de la estructura realizando un análisis modal real para determinar las características dinámicas básicas de una estructura ya que permite conocer las frecuencias y modos propios a los que la estructura tiende a vibrar. También es usado frecuentemente el análisis de respuesta en frecuencia dado que ofrece la respuesta permanente del sistema frente a cargas senoidales de frecuencia, amplitud y fase conocidas. El tercer tipo de análisis dinámico más empleado es análisis transitorio o de respuesta temporal que calcula la respuesta ante cargas variables con el tiempo.

Gran número de métodos de reducción de modelos se basan en las especiales características del problema de autovalores que resuelve el análisis modal. En primer lugar la superposición modal permite el paso de los desplazamientos del espacio físico al modal tomando como base los modos propios. En segundo lugar, la ortogonalidad de los modos propios asegura que son linealmente independientes unos de otros.

Dadas las peculiaridades de las grandes estructuras como es el caso de los modelos completos de ala de aeronave, en algunos casos el primer escalón del análisis dinámico que es el citado análisis modal se complica debido al gran número de datos de salida que proporciona por lo 
que es necesario buscar fórmulas que simplifiquen este problema.

Como se describe en [Qu, 2004] [Besselink, 2013], los métodos de reducción más comunes se basan en el método de la superposición modal [Rayleigh, 1945], según el cual un número limitado de modos de vibración de la estructura puede representar el comportamiento dinámico de la estructura completa a cambio de una pérdida de precision [Liu et al. , 2000] [Leontiev, n.d.] [Li \& Gunter, 1981]. Es muy común la implementación de mejoras y modificaciones a este método [Farstad \& Singh, 1996] como el método [Rixen, 2001] del aumento de la truncatura modal [Tham et al. , 2009], el método del desplazamiento modal o el método de la aceleración modal [Rixen, 2009]. Estas mejoras se encuentran limitadas por el procedimiento de selección de autovectores [Mourllion \& Birouche, 2013] y autovalores y por el importante coste computacional [Sutter et al. , 1986], [Bahai et al. , 2002] [Majkut, 2010], [Wilson, 2000] que implica el cálculo de los autovectores [Lázaro \& Pérez-Aparicio, 2013], [M.Lázaro et al. , 2012] de grandes estructuras, [Martinez-Agirre \& Elejabarrieta, 2011], [Zeng, 1995] que en el caso de las modificaciones estructurales multiplican la complejidad cuando se abordan análisis paramétricos. En muchos casos se trata de incorporar la información de los modos no considerados [Hermans et al. , 2000] [Roy \& Girard, 2005], modos residuales Otro de los más conocidos métodos de reducción de modelos es el debido a Guyan [Guyan, 1965]. La precisión de esta técnica depende en gran medida de la cuidadosa selección de los grados de libertad principales para los que se recomienda [Montáns \& Muñoz, 2013] la selección de los grados de libertad en la dirección principal del análisis, los que tengan mayor ratio $M_{i i} / K_{i i}$, que haya cierta separación entre los grados de libertad elegidos para que se considere la zona de la estructura con influencia en el análisis y por supuesto, el adecuado sentido físico de dichos grados de libertad. Adicionalmente a estas restricciones, al aplicar el método de Guyan las matrices pierden sus estructuras internas por lo que pueden resultar mucho más pobladas y ser mucho más costoso su manejo y el cálculo con ellas. Otros métodos como el de Rayleigh-Ritz [Wilson, 1985] y el de iteración de subespacios de Bathe [Bathe, 1996] [Chopra, 1995] tienen limitaciones similares.

Aunque el procedimiento que se describe en el presente trabajo puede usarse en conjunción con muchas de las técnicas de reducción de modelos y así aprovechar las ventajas de ambos enfoques, la diferencia fundamental es que no se propone la condensación del sistema de ecuaciones [R. J. Allemang, 1987] [P. Wallack, 1988] [Li \& He, 1999] [O'Callahan, 2003] como se hace en 
otros métodos de subestructuración [Craig \& Bampton, 1968] [Rixen, 2009] en los que las relaciones de compatibilidad deben asegurar el correcto comportamiento de la estructura completa [Hurty, 1965]. Por contra, se usa la información del sistema completo de ecuaciones aunque solo se procese la correspondiente a los grados de libertad de ciertos nodos que interesen al analista. Entre estos nodos suelen considerarse los que permitan obtener la respuesta de la estructura en una ubicación específica como los diversos lugares donde se proponga hacer la modificación estructural [Vibrant, 2014] [Schawarz et al. , 2007] [Schwarz \& Richardson, 1997]. El procedimiento permite el cambio de los parámetros físicos de masa, rigidez y amortiguamiento así como la adición de nuevos grados de libertad al modelo que permite simular adecuadamente elementos como amortiguadores o TMD (tuned mass dampers) así como modificaciones estructurales como refuerzos, rigidizaciones, vaciados, etc. por lo que está especialmente indicado para grandes estructuras cuya respuesta dinámica dispone de múltiples modos en un reducido ancho de banda. Además el método permite considerar cualquier tipo de amortiguamiento de la estructura así como la obtención de la respuesta tanto permanente como transitoria frente a cargas dinámicas.

Los programas comerciales [MSC.Sofware, 2012] [MSC.Software, 2004] más conocidos de análisis por el método de elementos finitos [Dassault, 2007] [ANSYS, 2013] no disponen de una herramienta [Benner et al. , 2009] tan potente, flexible y adaptable a la parametrización de modificaciones estructurales de grandes modelos como la que se describe en el presente trabajo.

\subsection{Amortiguamiento en estructuras}

El amortiguamiento [Mobley, 1999] [Harris, 1961] es el parámetro que representa la disipación de energía en una estructura. Suele recurrirse a su determinación experimental aunque se han desarrollado diferentes modelos matemáticos para su interpretación como el viscoso, el viscoelástico, de Coulomb, estructural, etc.

Como se ha comentado, para la extracción modal es habitual considerar despreciable el amortiguamiento ya que para la mayor parte de las estructuras el amortiguamiento estructural es pequeño, del orden del 1\% del amortiguamiento crítico. En los casos levemente amortiguados se asume que los modos son iguales a los del problema sin amortiguamiento a pesar de que las 
frecuencias se vean modificadas aunque sea ligeramente. Cuando no es elevado, es frecuente introducir el amortiguamiento en toda una estructura a través del amortiguamiento modal (generalmente entre 0.5 y $2 \%$ ) que viene dado por la relación del amortiguamiento respecto al amortiguamiento crítico del problema de un grado de libertad.

Una posibilidad para introducir este amortiguamiento es considerar la proyección modal de C como diagonal.

$$
\tilde{\boldsymbol{\Phi}}^{T} \mathbf{C} \tilde{\boldsymbol{\Phi}}=\operatorname{diag}\left(2 \zeta_{i} \omega_{i}\right), \quad i=1, . ., G
$$

Sin embargo, esta aproximación tiene difícil motivación física ya que liga el amortiguamiento de grados de libertad no conectado a través de sus términos de masa y rigidez. Por otro lado, que la matriz C sea completa complica su computación.

Por este motivo suele emplearse el amortiguamiento proporcional o de Rayleigh que considera la matriz C como proporcional a la matriz de masa y rigidez [Rayleigh, 1945].

$$
\mathbf{C}=\alpha \mathbf{K}+\beta \mathbf{M}
$$

Esta solución mejora el cálculo ya que la matriz pasa a tener una forma equivalente a las matrices que la generan siendo además sencillo obtener sus coeficientes a partir de dos frecuencias representativas pero muestra limitaciones en relación con las frecuencias de las que depende [Adhikari \& Phani, 2004].

Una generalización del método consiste en la linealización con más de dos frecuencias que se denomina amortiguamiento de Caughey

$$
\mathbf{C}=\mathbf{M} \sum_{k=0}^{p-1} a_{k}\left[\mathbf{M}^{-1} \mathbf{K}\right]^{k}
$$

que logra mejor aproximación manteniendo la posibilidad de desacoplar las ecuaciones del problema [Mortzfel \& Ma, 2011] pero sufre de mayores requerimientos de cálculo.

Cuando el amortiguamiento está localizado en zonas concretas de la estructura, como puede ser el caso de que se disponga de algún mecanismo específico, las consideraciones anteriores no son válidas y el planteamiento debe ser radicalmente distinto. Pueden hacerse aproximaciones 
para los términos proporcionales pero los no proporcionales deben construirse y considerarse adecuadamente [Papai et al. , 2012]. En este caso el problema pasa a ser de autovalores complejos [Gavin, 2014], también llamado problema de autovalores de amortiguamiento no clásico que requieren un tratamiento específico [Hoen, 2005] [Udwadia \& Esfandiari, 1990].

Los métodos habituales se basan en la formulación en la forma estándar no simétrica y en simétrica [Mathworks, 2013] [MSC.Software, 2004].

\subsection{Atenuación de excitaciones dinámicas}

Es frecuente que en numerosas ocasiones las cargas dinámicas introducidas en una estructura produzcan efectos no deseados bien sea en deformaciones/desplazamientos, velocidades o aceleraciones inducidas. Además de la salvaguarda de los límites de carga límite y carga última habituales para carga estática, ligados a las excitaciones dinámicas suelen considerarse otros requisitos adicionales. En algunos casos dichos requisitos tienen que ver con la integridad de la estructura analizada, como puede ser el caso de los fenómenos de resonancia a las frecuencias naturales o posibles interferencias físicas de algún modo propio. Sin embargo, pueden también considerarse otro tipo de requisitos que afectan al comportamiento dinámico de la estructura como pueden ser los impuestos por equipos, que pueden imponer limitaciones para su correcto funcionamiento, o incluso humanos como el nivel de confort que necesita un peatón al atravesar un puente o al estar en una terraza de un edificio.

Por casos como los enumerados, es usual la implementación de mecanismos amortiguadores específicos como los citados en el apartado anterior para la adecuación de las respuestas dinámicas de la estructura. Este tema ha sido abordado tradicionalmente desde diversos puntos de vista con lo que las soluciones que se le han propuesto han sido también variadas.

Si tomamos como ejemplo la excitación causada por un impacto determinado, se puede ver que [Pereira, 2001], [Brotons et al. , 1999], [Camarasa et al. , 2005] y [Johson \& Wilke, 2000] muestran ejemplos de amortiguación basada en elementos tradicionales como elementos elastoméricos, flejes, muelles metálicos y otros menos convencionales como las espumas y el panel con núcleo de nido de abeja. Esta última tecnología es analizada en detalle por Doengi [Doengi et al. , 1998] para los impactos debidos al aterrizaje de vehículos espaciales por su 
gran capacidad de absorción de energía basada en deformación irreversible y es que este es uno de los parámetros determinantes de los amortiguadores: su deformación frente a las cargas.

La desventaja de este último sistema es que es de un sólo uso y en muchas aplicaciones esto no es admisible siendo aconsejable que sea reversible para poder actuar repetidas veces, mientras que los sistemas tradicionales suelen penalizar la rigidez del conjunto.

Otra de las características de un mecanismo es el rango de fuerzas en el que evita que sean transmitidas a la estructura y finalmente, su capacidad de disipación de energía que está directamente relacionada con la magnitud mecánica llamada amortiguamiento.

En función de su modo de actuación, los amortiguadores [Choi \& Wereley, 2004] pueden clasificarse como:

- Pasivos: los que no requieren una fuente externa de energía para su actuación.

- Activos son sistemas que requieren la energía necesaria para su actuación. Suelen basarse en sistemas electromagnéticos, servoválvulas o piezocerámicos y pueden amortiguar en mayores anchos de banda que los pasivos. Sin embargo, pueden producir problemas de inestabilidad en el sistema de control por la falta de modelización de efectos dinámicos y no lineales y fallos del actuador o del sensor que son los que introducen energía al sistema. Además requieren un sistema de potencia, complejos algoritmos de control, sofisticadas configuraciones y tienen un alto coste de mantenimiento e instalación.

- Semiactivos generan fuerzas de control en respuesta a los movimientos detectados en el sistema. Es decir, aunque la fuerza de control se ajusta con el algoritmo adecuado, también dispone del mecanismo pasivo que opera aunque se produzca un fallo del sensor o actuador. Estos mecanismos no inyectan energía al sistema sino que ajustan de manera adecuada la fuerza resistiva o la fricción. Además como no producen problemas de inestabilidad, el consumo eléctrico se reduce drásticamente. A estos sistemas se les llama a veces inteligentes o "smart" aunque bajo este nombre se esconden numerosos conceptos ligeramente distintos. Cuando a una estructura dotada de sensores se le añade un sistema de actuación activo puede seleccionarse el sistema de control que gestiona este actuador. Es en realidad cuando este sistema de control es inteligente cuando se dice propiamente que la estructura es inteligente [Yang et al. , 2002]. 
Los sistemas de amortiguamiento pasivos tienen grandes desventajas: no se adaptan a las distintas cargas ni a su aplicación en el tiempo, sólo amortiguan en el rango de frecuencias para el que fueron diseñados, el amortiguador seleccionado debe soportar todas las cargas durante la vida útil de la estructura y la frecuencia de resonancia del conjunto debe ser menor que la de operación. Además no pueden formar distintas configuraciones estructurales como en el caso de los inteligentes que se adaptan a las condiciones de operación.

No obstante, la mayor complejidad de los amortiguadores activos e inteligentes los hace más caros y con más probabilidades de fallo.

Hay infinidad de tipos de amortiguadores pasivos y sus clasificaciones variadas, los típicos suelen ser:

- Viscoso.

- Elastomérico.

- De fricción.

- Neumático [Soulier et al. , 2003]

- De malla metálica [Acquaroli et al. , 2005]

- De espuma [Doengi et al. , 1998]

- Panel con núcleo de nido de abeja [Pereira, 2001]

En cambio, los amortiguadores activos [Baier \& Reindl, 2003], [Carpi et al. , 2005] suelen basarse en los siguientes principios:

- Rigidez variable, quizás sean el concepto más clásico dentro de este grupo. La rigidez de los elementos cambia según cambian las condiciones.

- Basados en fluidos controlables como los que disponen de orificios variables, la fuerza generada por el amortiguador es muy dependiente de su velocidad y deformación) o los magnetorreológicos (MR) en los que fuerza depende sólo de la corriente de alimentación del electroimán (fuerza arbitraria), bajos requisitos de consumo, simplicidad mecánica y fuerza de control grande y rango dinámico elevado. 
- Materiales magnetoestrictivos, con densidades de unos $9000 \mathrm{~kg} / \mathrm{m} 3$ y anchos de banda menores de $10 \mathrm{kHz}$.

- Materiales electroestrictivos, con rangos de temperatura admisibles entre 0 y $40^{\circ} \mathrm{C} \mathrm{y}$ anchos de banda de $100 \mathrm{kHz}$.

- Geles y polímeros biomiméticos

- Sensores de fibra óptica, en general como redes de difracción de Bragg.

- Aleaciones con memoria de forma (SMA). Masuda et al. [Masuda et al. , 2005] dan un ejemplo de uso de estos materiales en forma de tejido para la absorción de choques de baja velocidad. El tejido se deforma por la energía del impacto y recupera la forma original cuando se desee. Sin embargo, Masuda muestra experimentalmente que su baja capacidad de disipación de energía los hace desaconsejables para estas tareas.

- Piezoeléctricos poliméricos y cerámicos, que permiten la transformación de energía eléctrica a mecánica y viceversa, son usados cada vez más para el control de vibraciones [Sosnicki et al. , 2006] [Hanich et al. , 2001] [Lefevre et al. , 2003]. Son de los materiales inteligentes con mayor ancho de banda $(100 \mathrm{kHz})$ apropiados para fenómenos dinámicos y con rangos de temperatura admisibles de -20 a $120^{\circ} \mathrm{C}$.

De estos elementos habría que determinar el más adecuado según la aplicación a que vaya a destinarse y su posible implementación en el sistema completo; así como otros factores no tecnológicos como su disponibilidad comercial, coste, etc.

En la aplicación práctica concreta que se propone más adelante se empleará uno de estos sistemas como ejemplo de uso del método de aceleración de análisis paramétricos, de forma que las propiedades del mecanismo se determinen de forma adecuada y eficiente. 


\subsection{Objetivos y alcance de la tesis}

Esta tesis aborda el problema del manejo y solución de análisis dinámicos para grandes modelos de elementos finitos. Para ello, se propone implementar un método de reducción de grandes modelos estructurales basado en el subespacio modal complejo para un grupo reducido de grados de libertad. Estos grados de libertad permiten simular modificaciones estructurales locales por cambios en la masa, rigidez, amortiguamiento e incluso por la adición de nuevos grados de libertad a la estructura. Se revisa la teoría en la que se sustenta el procedimiento, aclarando su alcance de forma detallada y describiendo el procedimiento para su uso.

Posteriormente se aplica este método en diversos análisis paramétricos del modelo de elementos finitos del ala del UAV MILANO desarrollado por el Instituto Nacional de Técnica Aeroespacial. De esta forma, se estudia la optimización de los parámetros de la unión amortiguada de un equipo sujeto a la punta del ala para que se cumplan ciertos requisitos frente a las cargas dinámicas aplicadas.

Se propone validar los resultados obtenidos mediante su comparación con los resultados producidos por análisis dinámicos ejecutados de forma estándar por un programa comercial de elementos finitos. En dicha comparación se estudiará también la eficiencia del método en cuanto a necesidades de computación, tanto en tiempo de ejecución como en el tamaño de los archivos manejados.

Adicionalmente, se comprueba la flexibilidad del método para la selección del conjunto de parámetros en estudio estudiando la influencia de diversos factores en la precisión del método como pueden ser el amortiguamiento modal de la estructura, el número de modos retenidos en la truncutura modal o la precisión lograda por el barrido en frecuencia. 


\subsection{Estructura de la tesis}

En la presente introducción se han resumido ciertos aspectos de la reducción de modelos de elementos finitos para análisis dinámicos considerando sus aspectos más señalados. También se ha reflejado la influencia de la parametrización en dichos análisis y los distintos enfoques que reciben las modificaciones estructurales en estructuras sometidas a cargas dinámicas. Por último, se han citado diferentes procedimientos para la mencionada reducción de modelos y se han revisado los conceptos fundamentales en relación con el amortiguamiento en estructuras.

En el capítulo 2 se describe en detalle la teoría que sustenta el método en estudio, planteando las ecuaciones para la inclusión de modificaciones de masa, rigidez, amortiguamiento y nuevos grados de libertad. El sistema de ecuaciones resultante es resuelto tanto para la respuesta permanente como transitoria.

El tercer capítulo aborda la aplicación práctica de la técnica propuesta a un caso particular: el modelo de elementos finitos de la estructura del ala del vehículo aéreo no tripulado (UAV) MILANO desarrollado por el INTA. Además de su propia descripción, se introducen los requisitos de diseño que deben cumplirse al añadir un equipo en punta de ala izquierda. La carga de sustentación que una ráfaga continua produce sobre el ala y su procedimiento de análisis es el punto de partida para los análisis paramétricos con variaciones de masa, rigidez y amortiguamiento del sistema amortiguador, que une el equipo con la estructura del ala cuyos resultados son recogidos en el capítulo cuarto.

Finalmente se discuten las conclusiones más adecuadas y se recogen en el capítulo quinto. En dicho capítulo, se estudia la influencia de varios factores en la precisión del método y se analiza el cumplimiento de los objetivos de la tesis, tanto desde el punto de vista de la precisión del método como de su aplicabilidad y eficiencia. Por último, se proponen ciertas líneas de investigación relacionadas con los temas tratados y que pueden dar continuidad al presente trabajo.

Esta tesis se ha desarrollado con los medios facilitados, principalmente los programas MatLAB, MSC.Patran, MSC.Nastran y Scientific Workplace, por el Departamento de Estructuras y Materiales del Instituto Nacional de Técnica Aeroespacial - Esteban Terradas y del Departamento de Aeronaves y Vehículos Espaciales de la Escuela Técnica Superior de Ingenieros Aeronáuticos de la Universidad Politécnica de Madrid. 


\section{Capítulo 2}

\section{DESCRIPCIÓN DEL MÉTODO DE ACELERACIÓN DE ANÁLISIS DINÁMICOS}

En este capítulo se presenta el procedimiento para realizar un análisis por superposición modal en el cual solo se usa parte de la información de los modos. Posteriormente se modifican las matrices de la estructura para considerar modificaciones de masa, rigidez o amortiguamiento en nuevas ubicaciones. El método es exacto excepto cuando se aplica conjuntamente con la truncatura modal tal y como suele hacerse en el análisis de grandes estructuras. En ese caso, si las modificaciones no son relevantes en el comportamiento global de la estructura, afectando únicamente a zonas concretas, el procedimiento es suficientemente aproximado como se verá en apartados posteriores.

\subsection{Descomposición modal}

La conocida ecuación de dinámica estructural puede escribirse:

$$
\mathbf{M} \ddot{\mathbf{x}}+\mathbf{C} \dot{\mathbf{x}}+\mathbf{K} \mathbf{x}=\mathbf{P} f(t)
$$


donde $\mathbf{x}$ es el vector de desplazamiento de los nodos con dimensión de $G$ (número global de grados de libertad), $\mathbf{M}$ es la matriz de masas, $\mathbf{C}$ es la matriz de amortiguamiento, $\mathbf{K}$ es la matriz de rigidez, $\mathbf{P}$ es el vector de cargas y $f(t)$ es el coeficiente de la carga que es función del tiempo $(t)$. Se llama $\mathbf{X}$ al vector de coordenadas (con dimensión de número de nodos por número de grados de libertad por nodo).

Como es habitual se considera la superposición modal de los desplazamientos:

$$
\mathbf{x}(\mathbf{X}, t)=\mathbf{\Phi}(\mathbf{X}) \mathbf{u}(t) \Leftrightarrow \mathbf{x}(\mathbf{X}, t)=\sum_{\substack{k=1 \\ i=c(k)}}^{n} u_{c(k)}(t) \phi_{c(k)}(\mathbf{X})+\sum_{\substack{k=1 \\ i=n c(k)}}^{G-n} u_{n c(k)}(t) \phi_{n c(k)}(\mathbf{X})
$$

donde $u_{i}(t)$ son los desplazamientos o coordenadas modales y $\mathbf{\Phi}(\mathbf{X})$ es la matriz de modos, cuyas columnas, $\phi_{i}(\mathbf{X})$, son los modos de vibración de la estructura (reales si se cumplen ciertas condiciones que se especifican posteriormente), con $i=1, \ldots, G$. Esta matriz se ha particionado, siendo $n \leq G$ el número de modos que se consideran afectados por las modificaciones (grupo considerado), en general un número mucho menor que el número de modos real de la estructura (e.g. cientos frente cientos de miles), i.e.

$$
\underbrace{\mathbf{\Phi}_{c}}_{G \times n}=\left[\phi_{c(1)}, \phi_{c(2)}, \phi_{c(3)}, \ldots, \phi_{c(n)}\right] \quad \mathrm{y} \quad \phi_{i}=\left[\phi_{1 i}, \ldots, \phi_{G i}\right]^{T}
$$

y $G-n$ es el número de modos que no se considera afectado por las modificaciones. Hasta ahora no se ha impuesto ningún tipo de limitación, pudiendo ser la cantidad de elementos de dichos grupos cualquiera al igual que el orden. La contribución a la respuesta de este segundo grupo se añadirá como una suma al resultado final.

$$
\underbrace{\boldsymbol{\Phi}_{n c}}_{G \times(G-n)}=\left[\phi_{n c(1)}, \phi_{n c(2)}, \phi_{n c(3)}, \ldots, \phi_{n c(G-n)}\right]
$$

Si no hay amortiguamiento en la estructura (o este es proporcional), los modos son reales y la solución del problema de autovalores de la forma

$$
\left[\mathbf{K}-\omega_{i}^{2} \mathbf{M}\right] \phi_{i}=\mathbf{0}
$$


donde $\boldsymbol{\Omega}^{2}=\operatorname{diag}\left(\omega_{i}^{2}\right)$ es la matriz de autovalores reales sin amortiguamiento. Por sencillez de notación, durante el desarrollo teórico, se considerará la matriz modal completa normalizada $\tilde{\boldsymbol{\Phi}}$ y la matriz de autovalores completa $\tilde{\Omega}^{2}$ (ambas de tamaño $\left.G \times G\right)$, mientras que para referirse al grupo Considerado (de tamaño $G$ x $n$ ó $n$ x 1), se omitirán los subíndices hasta el resultado final. Como es bien sabido, al ser todos los modos independientes y su amplitud o norma no está determinada se puede realizar la normalización de modos usual con la masa $\tilde{\mathbf{\Phi}}^{T} \mathbf{M} \tilde{\boldsymbol{\Phi}}=\mathbf{I}$ de modo que la ecuación de movimiento queda como sigue

$$
\ddot{\mathbf{u}}+\tilde{\boldsymbol{\Phi}}^{T} \mathbf{C} \tilde{\boldsymbol{\Phi}} \dot{\mathbf{u}}+\boldsymbol{\Omega}^{2} \mathbf{u}=\tilde{\boldsymbol{\Phi}}^{T} \mathbf{P} f(t)
$$

Las condiciones para que los autovectores del sistema sean reales son que $\mathbf{C}$ se pueda escribir como función lineal de las matrices $\mathbf{K}, \mathbf{M}$ y sus inversas. También, bajo las condiciones habituales de bajo amortiguamiento en las estructuras, se puede considerar que la matriz de amortiguamiento modal, $\tilde{\boldsymbol{\Phi}}^{T} \mathbf{C} \tilde{\boldsymbol{\Phi}}$, es diagonal

$$
\tilde{\boldsymbol{\Phi}}^{T} \mathbf{C} \tilde{\boldsymbol{\Phi}}=\operatorname{diag}\left(2 \zeta_{i} \omega_{i}\right), \quad i=1, . ., G
$$

donde $\zeta_{i}$ es el "factor de amortiguamiento modal", correspondiente al del sistema de un grado de libertad del modo $i$ en el problema desacoplado resultante por ser las matrices del sistema, $\tilde{\mathbf{\Omega}}^{2}, \tilde{\boldsymbol{\Phi}}^{T} \mathbf{C} \tilde{\boldsymbol{\Phi}}$ y $\tilde{\boldsymbol{\Phi}}^{T} \mathbf{M} \tilde{\boldsymbol{\Phi}}=\mathbf{I}$, diagonales con el número de modos seleccionado de manera arbitraria. Si se denomina $\overline{\mathbf{C}}=\boldsymbol{\Phi}^{T} \mathbf{C} \boldsymbol{\Phi}$ a la matriz modal de amortiguamiento con la partición de $n$ modos indicada antes, la ecuación de movimiento en términos de las coordenadas modales correspondientes es:

$$
\ddot{\mathbf{u}}+\overline{\mathbf{C}} \dot{\mathbf{u}}+\boldsymbol{\Omega}^{2} \mathbf{u}=\boldsymbol{\Phi}^{T} \mathbf{P} f(t)
$$

Cabe resaltar que para conocer la respuesta de un grado de libertad determinado, únicamente es necesario considerar las componentes de los modos en ese grado de libertad. Por esto, en la práctica, se puede reducir adicionalmente el problema de superposición modal únicamente a aquellos $g<<G$ grados de libertad de interés y considerar $\boldsymbol{\Phi}$ como una matriz de dimensión $g \times n<<G \times G$. Por ejemplo, si $G=10^{6}$ grados de libertad, $G \times G=10^{12}$ números. Si $\mathrm{n}=100$ modos y $\mathrm{g}=1000$ grados de libertad, entonces $g \times n=10^{5}$ números. En el caso de simple 
precisión (4 bytes por número), las matrices de $G \times G$ quedarían de unos $4 \mathrm{~Tb}$ de datos y las de $g \times n$ de $0.4 \mathrm{Mb}$.

\subsection{Introducción de amortiguamiento adicional no proporcional.}

El uso de amortiguamiento proporcional para la estructura no modificada es debido a las ventajas conocidas en términos de eficiencia ya que los modos son reales y las ecuaciones desacopladas. Además la determinación experimental o teórica de la matriz de amortiguamiento real en una estructura grande sigue siendo una tarea complicada.

Sin embargo, hay ocasiones en las que el amortiguamiento proporcional no puede justificarse. Estos casos se deben a mecanismos localizados de atenuación o TMD (tuned mass dampers) usados para reducir las respuestas dinámicas no deseadas producidas por ciertos tipos de carga. En estos casos, la matriz de amortiguamiento no puede considerarse diagonal No obstante, solo algunos términos están afectados. Las necesarias pruebas y estudios paramétricos pueden realizarse sobre un problema muy reducido, considerando solo algunos grados de libertad de los nuevos modos y manteniendo el intuitivo amortiguamiento modal. Se considera la siguiente partición de la matriz de amortiguamiento.

$$
\mathbf{C}^{*}=\mathbf{C}+\mathbf{C}^{a}
$$

donde $\mathbf{C}=\operatorname{diag}\left(2 \zeta_{i} \omega_{i}\right)$ es la matriz previa de amortiguamiento proporcional, generalmente con amortiguamiento pequeño y $\mathbf{C}^{a}$ es la matriz de amortiguamiento no proporcional añadida debida a elementos amortiguadores localizados y cuyo amortiguamiento no será en general pequeño. La proyección en el subespacio modal resulta

$$
\overline{\mathbf{C}}^{*}=\boldsymbol{\Phi}^{T} \mathbf{C}^{*} \boldsymbol{\Phi}=\overline{\mathbf{C}}+\overline{\mathbf{C}}^{a}
$$

Suponiendo $N$ amortiguadores dentro del grupo de grados de libertad seleccionado $g$, con los siguientes valores de amortiguamiento: $\mathbf{c}=\left[c_{1}, c_{2}, \ldots, c_{N}\right]^{T}$. La matriz $\mathbf{C}^{a}$ no es diagonal porque los grados de libertad afectados por los elementos de c tienen términos no nulos. Por ejemplo, suponiendo solo un amortiguador que une dos grados de libertad de constante $c$, la matriz 
tendría la forma

$$
\mathbf{C}^{a}=\left[\begin{array}{llllllll}
\ddots & & & & & & \\
& 0 & 0 & 0 & 0 & 0 & \\
& 0 & c & 0 & -c & 0 & \\
& 0 & 0 & 0 & 0 & 0 & \\
0 & -c & 0 & c & 0 & \\
0 & 0 & 0 & 0 & 0 & \\
& & & & & & \ddots
\end{array}\right]
$$

Por lo tanto $\overline{\mathbf{C}}^{a}=\boldsymbol{\Phi}^{T} \mathbf{C}^{a} \boldsymbol{\Phi}$ se calcula como

$$
\begin{aligned}
\bar{C}_{i j}^{a} & =\sum_{p=1}^{g} \sum_{q=1}^{g} \phi_{p i} C_{p q}^{a} \phi_{q j} \\
& =\sum_{\substack{k=1 \\
r=\operatorname{dof} 1(k), s=\operatorname{dof} 2(k)}}^{N}\left(\phi_{r i} C_{r r}^{a} \phi_{r j}+\phi_{r i} C_{r s}^{a} \phi_{s j}+\phi_{s i} C_{s r}^{a} \phi_{r j}+\phi_{s i} C_{s s}^{a} \phi_{s j}\right)
\end{aligned}
$$

Donde solo las coordenadas modales de los grados de libertad afectados de los modos considerados tienen que ser considerados. La ecuación del movimiento reducida y proyectada es

$$
\ddot{\mathbf{u}}+\overline{\mathbf{C}}^{*} \dot{\mathbf{u}}+\mathbf{\Omega}^{2} \mathbf{u}=\boldsymbol{\Phi}^{T} \mathbf{P} f(t)
$$

Hay que destacar que $\overline{\mathbf{C}}^{*}$ ya no es diagonal pero su tamaño es mucho más pequeño que en el problema original $(n<<G)$. Para resolver este problema de autovalores complejo se puede reescribir el problema en términos de desplazamientos y velocidades modales:

$$
\mathbf{U}:=\left[\begin{array}{c}
\mathbf{u} \\
\mathbf{v}
\end{array}\right], \quad \dot{\mathbf{U}}:=\left[\begin{array}{c}
\dot{\mathbf{u}} \\
\dot{\mathbf{v}}
\end{array}\right]
$$

y $\ddot{\mathbf{u}}$ puede despejarse en la Ec. (2.13)

$$
\ddot{\mathbf{u}}=: \dot{\mathbf{v}}=-\overline{\mathbf{C}}^{*} \dot{\mathbf{u}}-\boldsymbol{\Omega}^{2} \mathbf{u}+\boldsymbol{\Phi}^{T} \mathbf{P} f(t)
$$


Por lo tanto, la Ec. (2.13) se escribe como sigue

$$
\left[\begin{array}{c}
\dot{\mathbf{u}} \\
\dot{\mathbf{v}}
\end{array}\right]=\underbrace{\left[\begin{array}{cc}
\mathbf{0} & \mathbf{I} \\
-\boldsymbol{\Omega}^{2} & -\overline{\mathbf{C}}^{*}
\end{array}\right]}\left[\begin{array}{c}
\mathbf{u} \\
\mathbf{v}
\end{array}\right]+\underbrace{\left[\begin{array}{c}
\mathbf{0} \\
\boldsymbol{\Phi}^{T} \mathbf{P}
\end{array}\right]} f(t)
$$

A

B

$\mathrm{O}$

$$
\dot{\mathbf{U}}=\mathbf{A} \mathbf{U}+\mathbf{B} f(t)
$$

Estas matrices se construyen teniendo en cuenta que el número de modificaciones es bajo, por lo que las matrices son casi diagonales con pocos términos fuera de la diagonal.

\subsection{Adición de elementos de masa y rigidez.}

La adición de masas y muelles en el sistema es simple y sigue un razonamiento similar al empleado para los términos de amortiguamiento. Para el caso de rigidez añadida, simplemente se considera la composición de la matriz de rigidez en dos términos: $\mathbf{K}$ con los términos no modificados y $\mathbf{K}^{a}$ con los añadidos

$$
\mathbf{K}^{*}=\mathbf{K}+\mathbf{K}^{a}
$$

La proyección sobre el subespacio de los modos originales considerados es

$$
\overline{\mathbf{K}}^{*}=\boldsymbol{\Phi}^{T} \mathbf{K}^{*} \boldsymbol{\Phi}=\boldsymbol{\Omega}^{2}+\boldsymbol{\Phi}^{T} \mathbf{K}^{a} \boldsymbol{\Phi}:=\boldsymbol{\Omega}^{2}+\overline{\mathbf{K}}^{a}
$$

donde $\overline{\mathbf{K}}^{a}$ puede ser obtenido de una forma análoga que en el caso de la matriz de amortiguamiento, es decir usando solo los grados de libertad de los modos relevantes

$$
\begin{aligned}
\bar{K}_{i j}^{a} & =\sum_{p=1}^{g} \sum_{q=1}^{g} \phi_{p i} K_{p q}^{a} \phi_{q j} \\
& =\sum_{\substack{k=1 \\
r=\operatorname{dof} 1(k), s=\operatorname{dof} 2(k)}}^{N}\left(\phi_{r i} K_{r r}^{a} \phi_{r j}+\phi_{r i} K_{r s}^{a} \phi_{s j}+\phi_{s i} K_{s r}^{a} \phi_{r j}+\phi_{s i} K_{s s}^{a} \phi_{s j}\right)
\end{aligned}
$$


Por lo tanto, la nueva matriz A en la Ec. (2.17) es

$$
\mathbf{A}=\left[\begin{array}{cc}
\mathbf{0} & \mathbf{I} \\
-\boldsymbol{\Omega}^{2}-\overline{\mathbf{K}}^{a} & -\overline{\mathbf{C}}^{*}
\end{array}\right]
$$

El mismo procedimiento se aplica a la matriz de masas mientras que la matriz $\mathbf{B}$ de la Ec. (2.16) permanece inalterada. Las masas añadidas se modelizan como una matriz de masas añadidas $\mathbf{M}^{a}$. Entonces

$$
\mathbf{M}^{*}=\mathbf{M}+\mathbf{M}^{a}
$$

y la proyección sobre el espacio reducido de modos considerados es

$$
\overline{\mathbf{M}}^{*}=\boldsymbol{\Phi}^{T} \mathbf{M}^{*} \boldsymbol{\Phi}=\mathbf{I}+\boldsymbol{\Phi}^{T} \mathbf{M}^{a} \boldsymbol{\Phi}:=\mathbf{I}+\overline{\mathbf{M}}^{a}
$$

donde $\overline{\mathbf{M}}^{a}$ se calcula de forma similar a $\overline{\mathbf{K}}^{a}$. La matriz final $\mathbf{A}$ es

$$
\mathbf{A}=\left[\begin{array}{cc}
\mathbf{0} & \mathbf{I}+\overline{\mathbf{M}}^{a} \\
-\mathbf{\Omega}^{2}-\overline{\mathbf{K}}^{a} & -\overline{\mathbf{C}}^{*}
\end{array}\right]
$$

\subsection{Adición de grados de libertad}

La adición de masa, rigidez o elementos amortiguadores puede hacer que sea necesario añadir también un pequeño número de $d$ grados de libertad no considerados en el modelo original. En este caso, se amplían los modos originales con los adicionales para considerar su influencia en la estructura original. Además se necesita considerar tantos vectores adicionales como grados de libertad considerados $d$ (por ejemplo para incluir nuevos modos locales). Se seleccionan estos nuevos vectores con valor unidad en su correspondiente grado de libertad.

$$
\hat{\boldsymbol{\Phi}}:=\left[\begin{array}{cc}
\boldsymbol{\Phi} & \mathbf{0}_{n \times d} \\
\mathbf{0}_{n \times d}^{T} & \mathbf{1}_{d \times d}
\end{array}\right]
$$


La matriz de rigidez ampliada original y proyectada es

$$
\hat{\boldsymbol{\Phi}}^{T} \hat{\mathbf{K}} \hat{\boldsymbol{\Phi}}=\left[\begin{array}{ll}
\boldsymbol{\Omega}^{2} & \mathbf{0} \\
\mathbf{0}^{T} & \mathbf{0}
\end{array}\right]=: \hat{\boldsymbol{\Omega}}^{2}
$$

y de forma similar las matrices ampliadas de masa y amortiguamiento

$$
\begin{gathered}
\hat{\boldsymbol{\Phi}}^{T} \hat{\mathbf{M}} \hat{\boldsymbol{\Phi}}=\left[\begin{array}{cc}
\boldsymbol{\Phi}^{T} \mathbf{M} \boldsymbol{\Phi} & \mathbf{0} \\
\mathbf{0}^{T} & \mathbf{0}
\end{array}\right]=\left[\begin{array}{cc}
\mathbf{I} & \mathbf{0} \\
\mathbf{0}^{T} & \mathbf{0}
\end{array}\right]=: \hat{\mathbf{I}} \\
\hat{\boldsymbol{\Phi}}^{T} \hat{\mathbf{C}} \hat{\boldsymbol{\Phi}}=\left[\begin{array}{cc}
\overline{\mathbf{C}} & \mathbf{0} \\
\mathbf{0}^{T} & \mathbf{0}
\end{array}\right]=: \hat{\overline{\mathbf{C}}}
\end{gathered}
$$

Los términos añadidos de amortiguamiento, rigidez o masa están incluidos como antes pero considerando el nuevo grupo de vectores y grados de libertad

$$
\hat{K}_{i j}^{a}=\sum_{p=1}^{g+d} \sum_{q=1}^{g+d} \hat{\phi}_{p i} K_{p q}^{a} \hat{\phi}_{q j}
$$

donde $\hat{\phi}_{p i}=0$ para $p>n$ excepto $\hat{\phi}_{p p}=1$. Finalmente, la $\hat{\mathbf{A}}$ ampliada es

$$
\hat{\mathbf{A}}=\left[\begin{array}{cc}
\hat{\mathbf{0}} & \hat{\mathbf{I}}+\widehat{\overline{\mathbf{M}}}^{a} \\
-\hat{\mathbf{\Omega}}^{2}-\widehat{\overline{\mathbf{K}}}^{a} & -\widehat{\overline{\mathbf{C}}}^{*}
\end{array}\right]
$$

En los apartados siguientes se omite el sombrero que denota los términos ampliados porque no hay diferencia en el procedimiento una vez que las matrices han sido ampliadas.

\subsection{Solución del problema general}

Se considera el nuevo sistema Ec. (2.17). Suponiendo soluciones del tipo

$$
\mathbf{U}(\mathbf{X}, t)=\mathbf{\Psi}(\mathbf{X}) \xi(t)
$$


donde $\boldsymbol{\Psi}$ es la matriz de transformación que convierte los modos reales del modelo original a los complejos del modelo modificado. A la vez son los modos complejos del nuevo problema de autovalores

$$
\mathbf{A} \Psi=\boldsymbol{\Lambda} \Psi \quad \text { with } \boldsymbol{\Lambda}=\operatorname{diag}\left(\lambda_{i}\right)
$$

El vector $\xi$ contiene los multiplicadores modales y $\lambda_{i}$ son los $2 n$ autovalores complejos del nuevo problema. Como es sabido, los modos complejos y los autovalores complejos de este tipo de problemas aparecen en pares conjugados. Ec. (2.17) se escribe ahora en la forma

$$
\mathbf{\Psi} \dot{\xi}=\mathbf{A} \boldsymbol{\Psi} \xi+\mathbf{B} f(t)
$$

Como $\mathbf{A}$ es no simétrica, un nuevo conjunto de vectores $\boldsymbol{\Gamma}$ es introducido

$$
\boldsymbol{\Gamma}^{T} \mathbf{\Psi} \dot{\xi}-\boldsymbol{\Gamma}^{T} \mathbf{A} \boldsymbol{\Psi} \xi=\boldsymbol{\Gamma}^{T} \mathbf{B} f(t)
$$

de forma que $\gamma_{i}^{T} \mathbf{A} \psi_{j}=\lambda_{j} \gamma_{i}^{T} \psi_{j}$, donde $\gamma_{i}$ son las columnas de $\boldsymbol{\Gamma}$ (los autovectores de $\mathbf{A}^{T}$ ) y $\psi_{i}$ son los de $\boldsymbol{\Psi}$.

$$
\boldsymbol{\Gamma}^{T} \mathbf{A} \Psi=\Lambda \boldsymbol{\Gamma}^{T} \boldsymbol{\Psi}
$$

Para simplificar Ec.(2.33) se realiza la normalización habitual. Usando

$$
\begin{aligned}
& \gamma_{i}^{T}\left(\mathbf{A} \psi_{j}\right)=\gamma_{i}^{T} \lambda_{j} \psi_{j} \\
& \left(\gamma_{i}^{T} \mathbf{A}\right) \psi_{j}=\lambda_{i} \gamma_{i}^{T} \psi_{j}
\end{aligned}
$$

se obtiene

$$
0=\left(\lambda_{j}-\lambda_{i}\right) \gamma_{i}^{T} \psi_{j} \Rightarrow \text { si } \lambda_{i} \neq \lambda_{j} \text { entonces } \gamma_{i}^{T} \psi_{j}=0
$$

así $\boldsymbol{\Gamma}^{T} \boldsymbol{\Psi}$ es una matriz diagonal ( $\gamma_{i}$ y $\psi_{j}$ son ortogonales). Entonces se puede redefinir los autovectores para obtener $\boldsymbol{\Gamma}^{\mathbf{T}} \mathbf{\Psi}=\mathbf{I}$; por ejemplo

$$
\psi_{i} \leftarrow \frac{1}{\left(\gamma_{i}^{T} \psi_{i}\right)} \psi_{i}
$$


y Ec. (2.33) toma ahora la forma

$$
\dot{\xi}-\mathbf{\Lambda} \xi=\boldsymbol{\Gamma}^{T} \mathbf{B} f(t)
$$

que es ahora un sistema de $2 n$ ecuaciones diferenciales desacopladas

$$
\dot{\xi}_{i}-\lambda_{i} \xi_{i}=b_{i} f(t) \quad \operatorname{con} b_{i}=\gamma_{i}^{T} \mathbf{B}
$$

cuya solución es

$$
\xi_{i}(t)=e^{-h}\left[\int_{0}^{t} e^{-\lambda_{i} \tau} b_{i} f(\tau) d \tau+\text { const }\right] \quad \operatorname{con} h=\int_{0}^{t}\left(-\lambda_{i}\right) d t=-\lambda_{i} t
$$

Para condiciones iniciales no nulas, $\xi_{i}(0)=\xi_{i 0} \neq 0$, la solución es como sigue:

$$
\begin{aligned}
\xi_{i}(t) & =b_{i} \int_{0}^{t} e^{\lambda_{i}(t-\tau)} f(\tau) d \tau+\xi_{i}^{0}(t) \\
& =b_{i} \int_{0}^{t} e^{\lambda_{i}(t-\tau)} f(\tau) d \tau+e^{\lambda_{i} t} \xi_{i 0}
\end{aligned}
$$

Si se considera a $f(t)$ como una superposición de $M$ funciones trigonométricas (como puede ser aplicando la transformada de Fourier a una excitación general)

$$
f(t)=\sum_{m=1}^{M} a_{m} \sin \left(\varpi_{m} t+\varphi_{m}\right)
$$

Entonces la solución se puede obtener por superposición a partir de

$$
\xi_{i}(t)=\sum_{m=1}^{M} \bar{\xi}_{i m}(t)+\xi_{i}^{0}(t)
$$

donde, para cada término $m$, la solución es 


$$
\begin{aligned}
\bar{\xi}_{i m}(t) & =a_{m} b_{i} \int_{0}^{t} e^{(t-\tau) \lambda_{i}} \sin \left(\varpi_{m} \tau+\varphi_{m}\right) d \tau \\
& =\frac{a_{m} b_{i}}{\lambda_{i}^{2}+\varpi_{m}^{2}}\left[\varpi_{m}\left(\cos \varphi_{m}\right) e^{t \lambda_{i}}+\lambda_{i}\left(\sin \varphi_{m}\right) e^{t \lambda_{i}}\right. \\
& \left.-\varpi_{m} \cos \left(\varphi_{m}+t \varpi_{m}\right)-\lambda_{i} \sin \left(\varphi_{m}+t \varpi_{m}\right)\right]
\end{aligned}
$$

$\mathrm{y}$

$$
\xi_{i}^{0}(t)=e^{\lambda_{i} t} \xi_{i 0} \quad \text { con } \quad \xi_{0}=\mathbf{\Psi}^{-1} \mathbf{U}(\mathbf{X}, 0)
$$

En general, el interés reside en la parte permanente de la respuesta. En tal caso, se puede descomponer el autovalor complejo en sus partes real e imaginaria

$$
\lambda_{i}=\sigma_{i}+j \omega_{d i}
$$

de tal modo que

$$
e^{\lambda_{i} t}=e^{\sigma_{i} t} e^{j \omega_{d i} t}
$$

$\mathrm{y}$

$$
\begin{aligned}
\bar{\xi}_{i m}(t) & =\frac{a_{m} b_{i}}{\lambda_{i}^{2}+\varpi_{m}^{2}}[\underbrace{-\varpi_{m} \cos \left(\varphi_{m}+t \varpi_{m}\right)-\lambda_{i} \sin \left(\varphi_{m}+t \varpi_{m}\right)}_{\text {permanente }} \\
& \underbrace{\left.+\varpi_{m}\left(\cos \varphi_{m}\right) e^{\sigma_{i} t} e^{j \omega_{d i} t}+\lambda_{i}\left(\sin \varphi_{m}\right) e^{\sigma_{i} t} e^{j \omega_{d i} t}\right]}_{\text {transitoria }}
\end{aligned}
$$

Para respuestas acotadas con $\sigma<0$, se tiene para la respuesta permanente

$$
\begin{aligned}
\bar{\xi}_{i m}^{\infty}:=\bar{\xi}_{i m}(t \rightarrow \infty) & = \\
& =\frac{-a_{m} b_{i}}{\lambda_{i}^{2}+\varpi_{m}^{2}}\left[\varpi_{m} \cos \left(t \varpi_{m}+\varphi_{m}\right)+\lambda_{i} \sin \left(t \varpi_{m}+\varphi_{m}\right)\right] \\
& =\bar{A}_{i m} \sin \left(t \varpi_{m}+\varphi_{m}\right)+\bar{B}_{i m} \cos \left(t \varpi_{m}+\varphi_{m}\right)
\end{aligned}
$$


y la respuesta transitoria es $\xi_{i}^{t}:=\xi_{i}^{0}+\sum_{m=1}^{M} \bar{\xi}_{i m}^{t}$, donde $\xi_{i}^{0}=e^{\lambda_{i} t} \xi_{i 0}$ y $\bar{\xi}_{i m}^{t}$ es

$$
\bar{\xi}_{i m}^{t}:=-e^{\sigma_{i} t} e^{j \omega_{d i} t}\left[\bar{B}_{i m} \cos \varphi_{m}+\bar{A}_{i m} \sin \varphi_{m}\right]
$$

con las amplitudes

$$
\bar{A}_{i m}=\frac{-b_{i} \lambda_{i}}{\lambda_{i}^{2}+\varpi_{m}^{2}} a_{m} \quad \text { y } \quad \bar{B}_{i m}=\frac{-b_{i} \varpi_{m}}{\lambda_{i}^{2}+\varpi_{m}^{2}} a_{m}
$$

De este modo, las operaciones para la respuesta permanente se pueden obtener en función de estas amplitudes

$$
\xi_{i}^{\infty}(t)=\sum_{m=1}^{M} \bar{\xi}_{i m}^{\infty}(t) \Rightarrow \xi^{\infty}=\sum_{m=1}^{M} \bar{\xi}_{m}^{\infty}=\sum_{m=1}^{M}\left[\overline{\mathbf{A}}_{m} \sin \left(t \varpi_{m}+\varphi_{m}\right)+\overline{\mathbf{B}}_{m} \cos \left(t \varpi_{m}+\varphi_{m}\right)\right]
$$

donde $\overline{\mathbf{A}}_{m}$ y $\overline{\mathbf{B}}_{m}$ son vectores, siendo $\bar{A}_{i m}$ el término correspondiente al modo $i$. De este modo, si se recupera la expresión de la Ec. (2.30) :

$$
\begin{gathered}
\mathbf{U}=\boldsymbol{\Psi}(\mathbf{X}) \xi(t) \\
\mathbf{U}^{\infty}=\left[\begin{array}{l}
\mathbf{u} \\
\mathbf{v}
\end{array}\right]^{\infty}=\sum_{m=1}^{M}\left(\boldsymbol{\Psi} \overline{\mathbf{A}}_{m} \sin \left(t \varpi_{m}+\varphi_{m}\right)+\boldsymbol{\Psi} \overline{\mathbf{B}}_{m} \cos \left(t \varpi_{m}+\varphi_{m}\right)\right)
\end{gathered}
$$

y a partir de la Ec. (2.14), se puede realizar la siguiente partición:

$$
\boldsymbol{\Psi} \overline{\mathbf{A}}_{m}=\left[\begin{array}{c}
\left(\boldsymbol{\Psi} \overline{\mathbf{A}}_{m}\right)^{U} \\
\left(\boldsymbol{\Psi} \overline{\mathbf{A}}_{m}\right)^{L}
\end{array}\right] ; \quad \boldsymbol{\Psi} \overline{\mathbf{B}}_{m}=\left[\begin{array}{c}
\left(\boldsymbol{\Psi} \overline{\mathbf{B}}_{m}\right)^{U} \\
\left(\boldsymbol{\Psi} \overline{\mathbf{B}}_{m}\right)^{L}
\end{array}\right]
$$

Entonces

$$
\mathbf{x}^{\infty}=\mathbf{\Phi} \mathbf{u}^{\infty}=\sum_{m=1}^{M}[\underbrace{\boldsymbol{\Phi}\left(\boldsymbol{\Psi} \overline{\mathbf{A}}_{m}\right)^{U}}_{:=\hat{\mathbf{a}}_{m}^{\infty}} \sin \left(t \varpi_{m}+\varphi_{m}\right)+\underbrace{\boldsymbol{\Phi}\left(\boldsymbol{\Psi} \overline{\mathbf{B}}_{m}\right)^{U}}_{:=\hat{\mathbf{b}}_{m}^{\infty}} \cos \left(t \varpi_{m}+\varphi_{m}\right)]
$$

Adicionalmente, la parte transitoria de la respuesta se puede calcular a partir de las ecua- 
ciones $(2.54)$ y $(2.55)$ :

$$
\begin{aligned}
\mathbf{x}^{t}=\boldsymbol{\Phi} \mathbf{u}^{t} & =-\sum_{m=1}^{M}[\underbrace{\boldsymbol{\Phi}\left(\boldsymbol{\Psi} \mathbf{e}^{\boldsymbol{\Lambda t}} \overline{\mathbf{A}}_{m}\right)^{U} \sin \varphi_{m}}_{:=\hat{\mathbf{a}}_{m}^{t}(t)}+\underbrace{\boldsymbol{\Phi}\left(\boldsymbol{\Psi} \mathbf{e}^{\boldsymbol{\Lambda t}} \overline{\mathbf{B}}_{m}\right)^{U} \cos \varphi_{m}}_{:=\hat{\mathbf{b}}_{m}^{t}(t)}] \\
& +\underbrace{\boldsymbol{\Phi}\left(\boldsymbol{\Psi} \mathbf{e}^{\boldsymbol{\Lambda t}} \boldsymbol{\Psi}^{-1}\left[\begin{array}{l}
\mathbf{u}_{0} \\
\mathbf{v}_{0}
\end{array}\right]\right)}_{:=\hat{\mathbf{c}}^{t}(t)}
\end{aligned}
$$

Finalmente, para el caso más general, añadiendo la contribución de los modos no considerados para las modificaciones, se obtiene:

$$
\mathbf{x}(t)=\mathbf{x}^{t}(t)+\mathbf{x}^{\infty}(t)+\sum_{\substack{k=1 \\ i=n c(k)}}^{G-n} u_{n c(k)}(t) \phi_{n c(k)}(\mathbf{X})
$$




\section{Capítulo 3}

\section{DESCRIPCIÓN DEL CASO DE ANÁLISIS}

\subsection{Descripción del modelo}

El ala que se presenta como modelo estructural es un modelo de una estructura real con prototipos ya fabricados, desarrollada dentro del programa MILANO que se introduce a continuación.

Es un sistema de reconocimiento, vigilancia y adquisición de blancos utilizando vehículos aéreos no tripulados capaces de realizar misiones a altitud media (7000 m) durante prolongados periodos de tiempo (hasta 20h). Los vehículos aéreos están equipados con cámaras CCD, con sensor compuesto por células fotoeléctricas, y FLIR (infrarrojo de barrido frontal), y tendrán capacidad para integrar un radar de apertura sintética, que procesa mediante algoritmos la información detectada y la combina para mejorar sus características. Durante el desarrollo del proyecto, se integrarán en el MILANO los desarrollos realizados por el INTA relativos al Radar de Apertura Sintética (SAR). El diseño permitirá asimismo la integración de un SAR comercial en caso de ser requerido por un futuro usuario.

Los vehículos aéreos y los sensores de observación son controlados y monitorizados en tiempo real desde una estación de control en tierra. Las comunicaciones ascendentes (telecomando) y descendentes (telemedida de datos e imágenes), entre la estación de control y los vehículos aéreos, serán realizadas tanto por enlace en línea de vista radio eléctrica como por enlace satélite. De esta manera, el alcance de la aeronave no estará limitado a operaciones en línea de 
vista radio eléctrica.

La geometría de la aeronave MILANO se ha diseñado de modo que presente una baja observabilidad a radares terrestres.

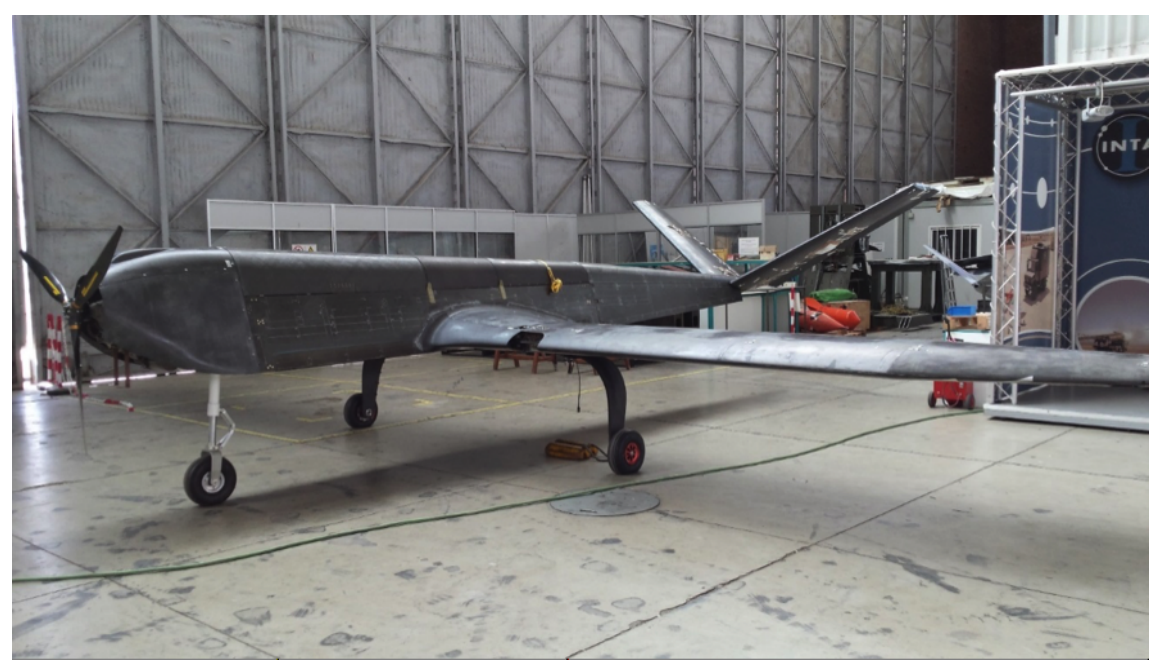

Figura 3-1: Prototipo MILANO (cortesía INTA)

Como características principales de la aeronave cabe destacar que se trata de una aeronave no tripulada, con un peso máximo al despegue de $896 \mathrm{~kg}$, techo de operación a $7620 \mathrm{~m}$, y envergadura de $12.5 \mathrm{~m}$. La estructura primaria se ha realizado en material compuesto de resina epoxi y fibra de carbono, con diseños de estructura semimonocasco y paneles sándwich, y con uniones metálicas y uniones encoladas reforzadas con remaches. El método de fabricación es mediante preimpregnados curados a con bolsa de vacío, temperatura controlada y a presión ambiente. Por requisitos de transporte indicados en la especificación se trata de una estructura modular (el ala tiene una unión de montaje/desmontaje sencillo con el fuselaje y el propio ala está dividido en una zona central y dos semialas exteriores). Por último, tiene una vida útil estimada de 15 años o 7500 horas de vuelo. 


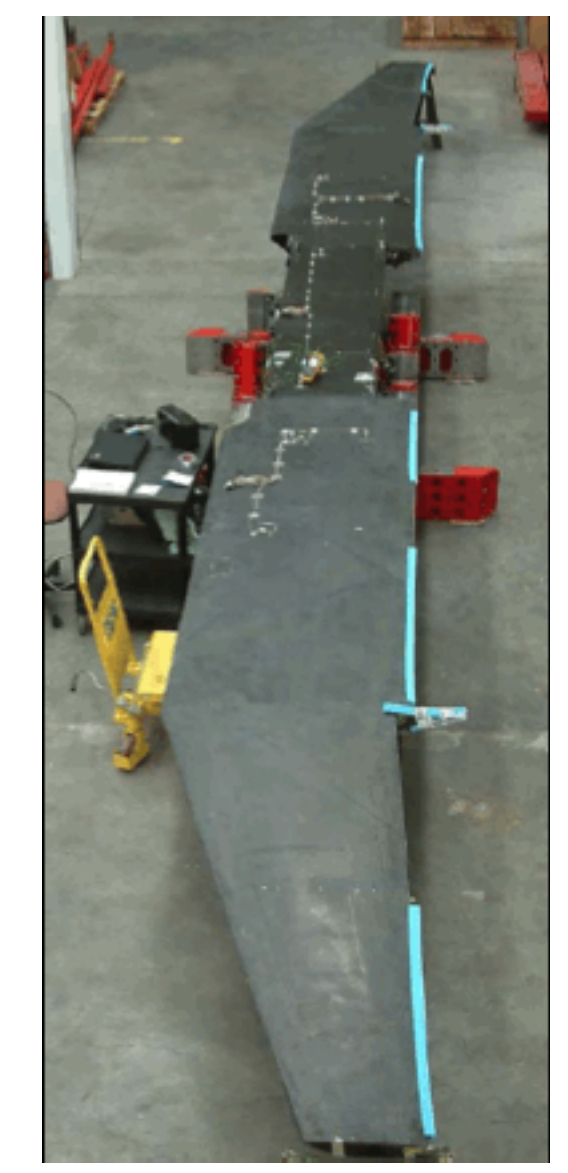

Figura 3-2: Prototipo ala MILANO (cortesía INTA)

\subsection{Modelo del ala}

El ala completa del UAV cuenta con un cajón central y dos cajones exteriores con estrechamiento, todos ellos constituidos por paneles rigidizados cerrados por dos largueros de borde de ataque y de salida, y con costillas, todos estos elementos de material compuesto. El número de larguerillos es de 3 en el intradós del ala y de 5 en el extradós, tienen sección en $\mathrm{Z}$ y se encuentran coencolados por adhesivo film. La zona con estrechamiento sustituye los paneles rigidizados por paneles sándwich de núcleo de espuma. Los cajones se unen entre sí con bulones insertados en herrajes metálicos en la zona de los largueros delanteros y traseros del ala. Hay 8 costillas por semiala, una doble correspondiente a la zona de unión entre cajón central y cajón exterior. Una imagen del modelo de una semiala puede verse en la figura 3-3.

La estructura se apoya en los herrajes de unión ala - fuselaje, simulando las condiciones de 
unión al fuselaje.

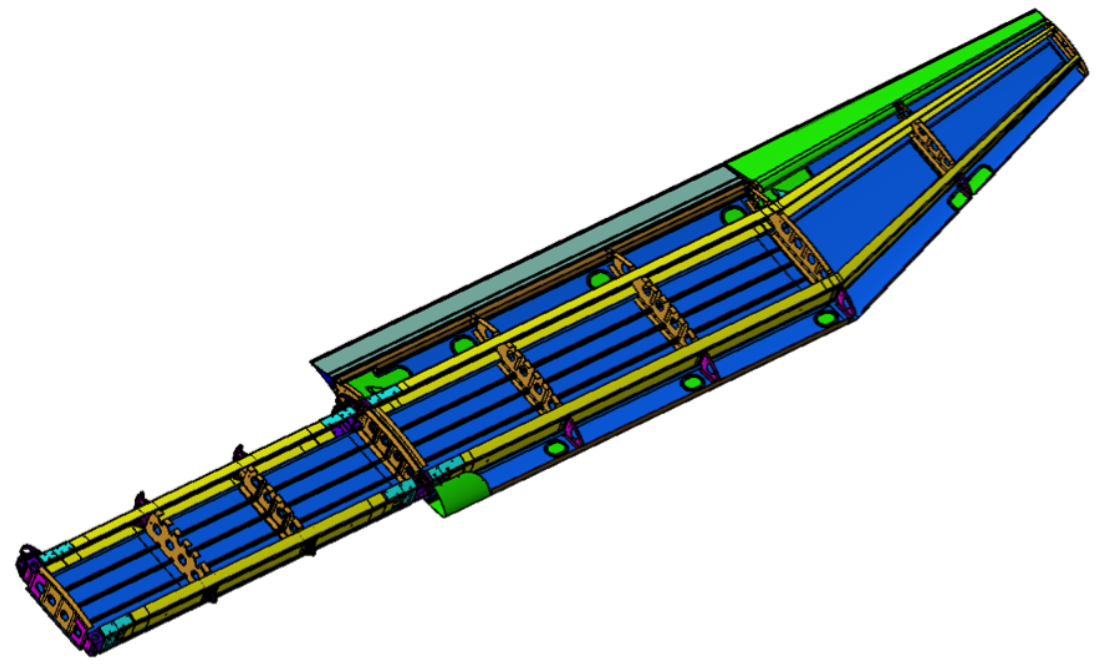

Figura 3-3: Modelo FEM semiala MILANO (cortesía INTA)

El MEF simula la estructura del ala descrita anteriormente. Las estadísticas del modelo son las siguientes:

- $\quad$ Número de nodos: 96235

- $\quad$ Número de elementos: 96700

- $\quad$ Número de MPC: 40 (tipos RBAR, RBE2, RBE3)

- Número de materiales: 7 de los cuales 5 isótropos y 2 ortótropos.

- $\quad$ Tipos de Elemento: barra (BAR2), placa (QUAD4, TRIA3)

Puede verse una representación del modelo completo en la figura 3-4.

El modelo de elementos finitos del ala se encuentra organizado jerárquicamente en una serie de grupos atendiendo a las diferentes partes de la estructura y orientados a las diferentes propiedades de los elementos. Por otro lado se ha establecido una numeración para nodos y elementos consistente en lo posible con la clasificación realizada por grupos. Debido al gran tamaño del modelo, se necesita una elevada capacidad de almacenamiento en memoria en el ordenador que se emplee. El mero cálculo de los autovalores del problema requiere un ordenador lo suficientemente potente como para manejar archivos de tamaños de gigabytes. 


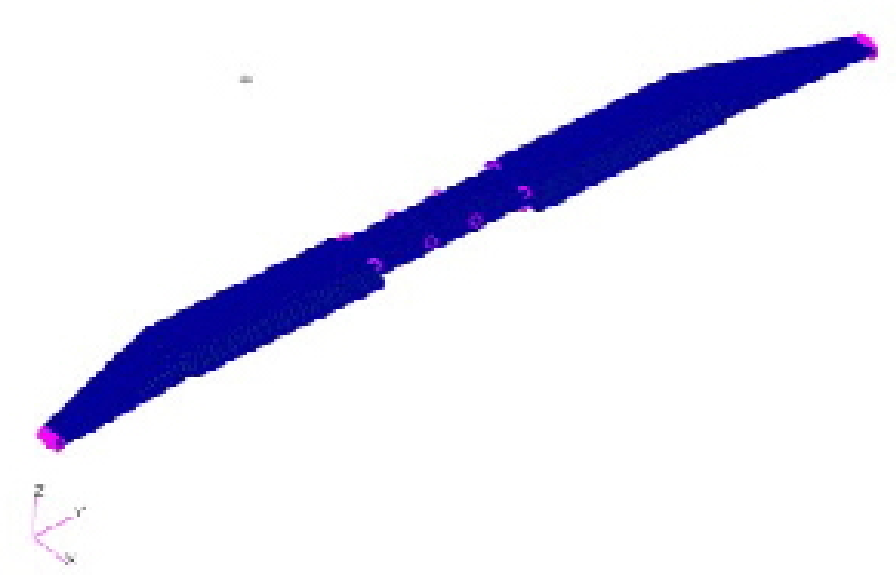

Figura 3-4: FEM completo del ala (cortesía INTA)

\subsection{Materiales y propiedades}

Los materiales empleados son materiales de uso habitual en estructuras aeronáuticas, entre los que se encuentran el material compuesto (usando como componentes resina Epoxi, fibra de carbono y espuma Rohacell, esta última empleada como núcleo en las zonas de estructura sandwich), Al 7075 T6 y acero 15-5PH. Para los elementos de unión se han empleado remaches calificados para uso aeronáutico (Cherry y HiLok), adhesivo film y adhesivo en pasta.

En las figuras 3-6, 3-7, 3-5 y se muestran esquemas aclaratorios de las propiedades implementadas en el modelo (la numeración se corresponde con la de los laminados en los planos de fabricación).

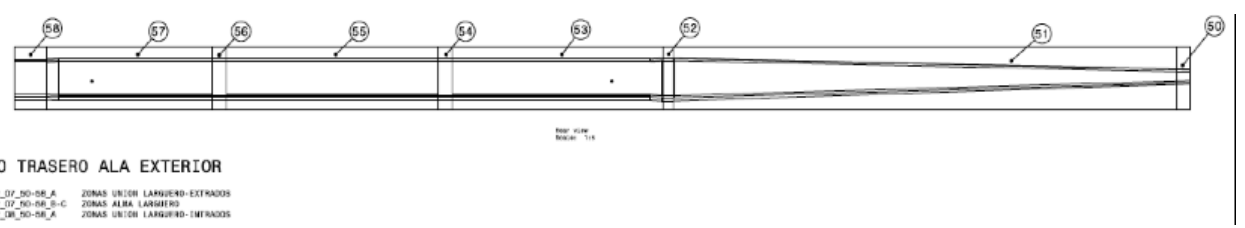

Figura 3-5: Esquema de las capas de los largueros del ala exterior (cortesía INTA) 


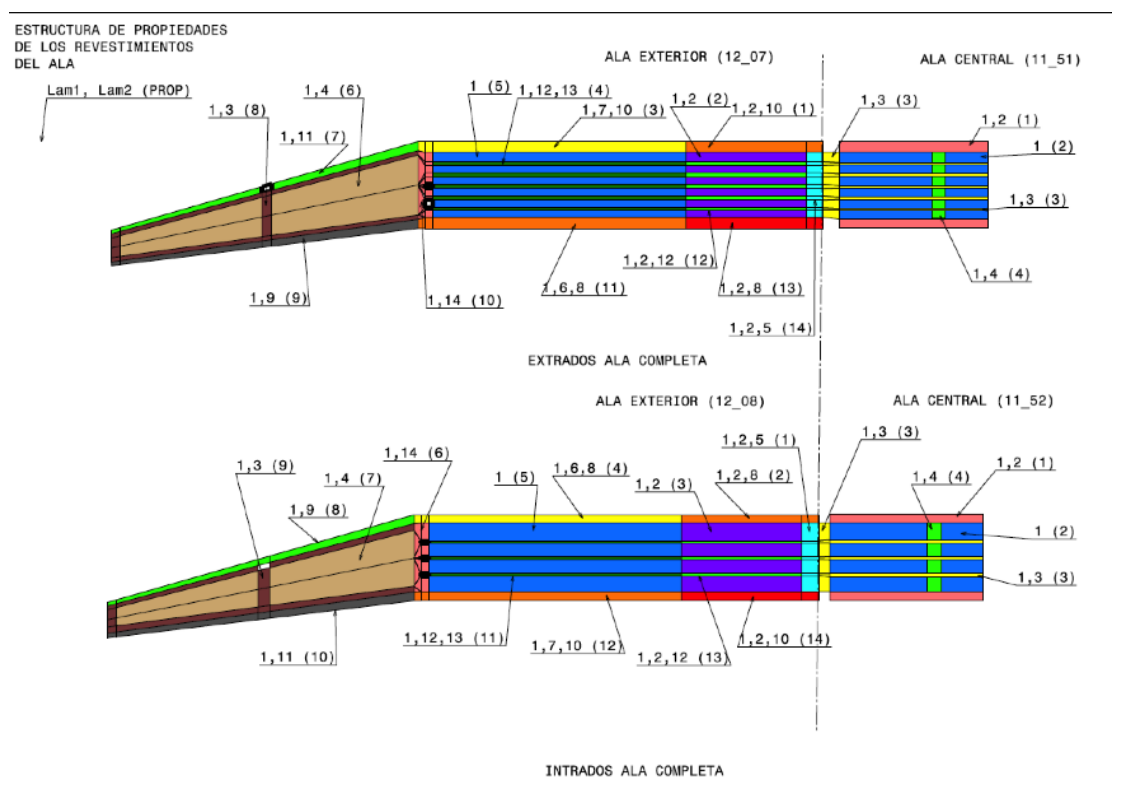

Figura 3-6: Esquema de las propiedades del modelo (cortesía INTA)

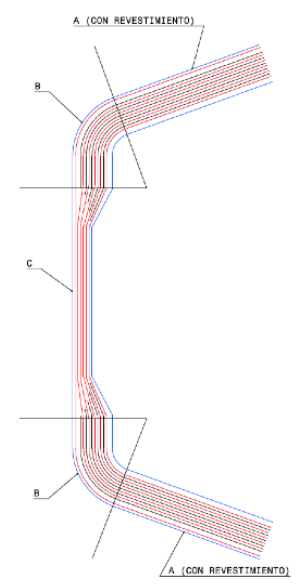

Figura 3-7: Esquema de las capas de los largueros del ala central (cortesía INTA) 


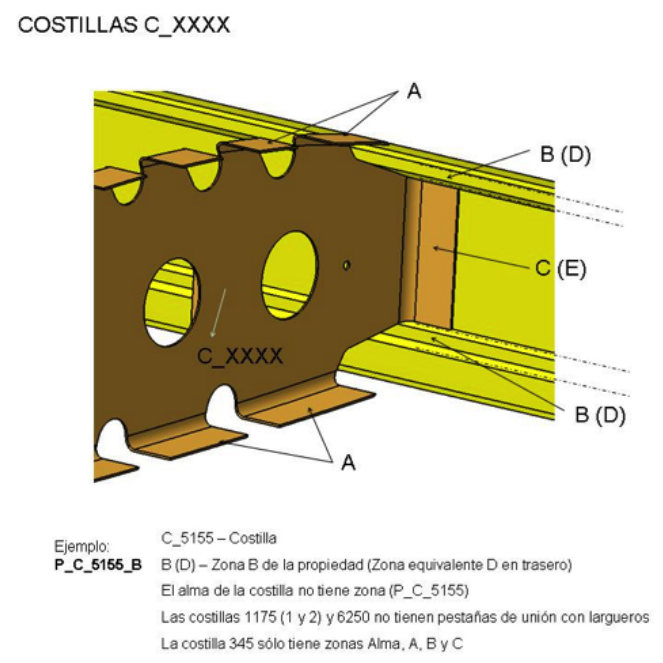

Figura 3-8: Esquema ensamblaje de las costillas (cortesía INTA)

\subsection{Condiciones de contorno}

Las condiciones de contorno empleadas son las siguientes:

Se han impedido los desplazamientos correspondientes a los restringidos por los bulones situados en los herrajes de unión ala central - fuselaje:

- Herrajes traseros. Se restringe desplazamiento en el plano del herraje (ejes Y, Z absolutos) en un nodo central. Ver figura 3-15. 
P_11/12 - HERRAJE ALA CENTRAL DELANTERO/TRASERO

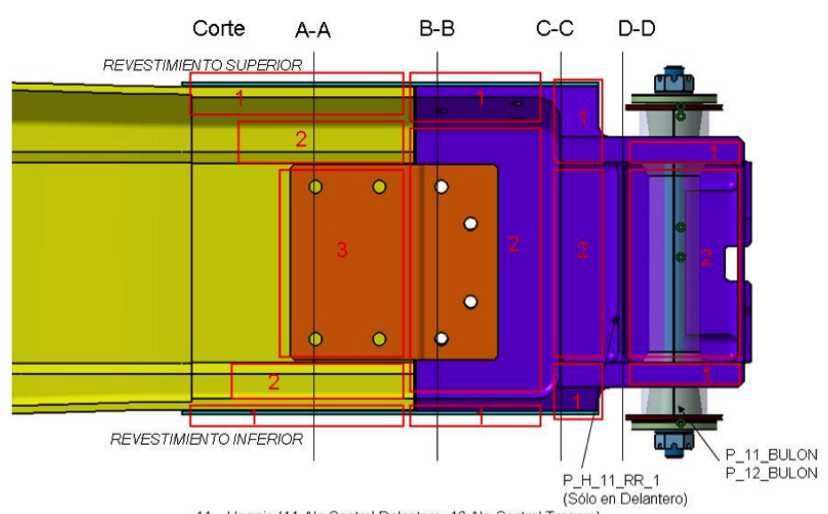

Ejemplo: 11 - Herraje (11 Ala Central Delantero, 12 Ala Central Trasero)

$$
\text { 1. Zona }
$$

Figura 3-9: Herraje ala central delantero/trasero (cortesía INTA)

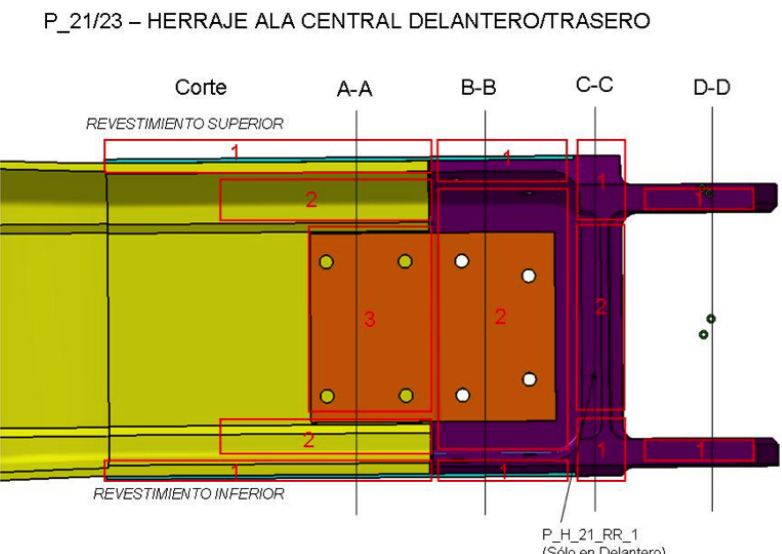

$\begin{array}{ll}\text { Ejemplo: } & 21 \text { - Herraje (21 Ala Exterior Delantero, } 23 \text { Ala Exterior Trasero) } \\ \text { P_11_AA_1 AA - Corte } & \end{array}$

Figura 3-10: Herraje ala exterior delantero/trasero (cortesía INTA) 


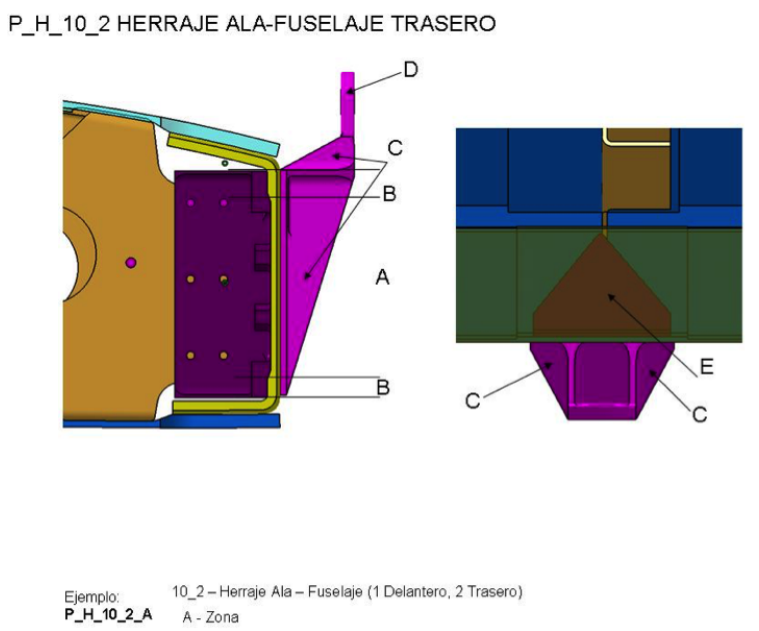

Figura 3-11: Herraje ala-fuselaje trasero (cortesía INTA)

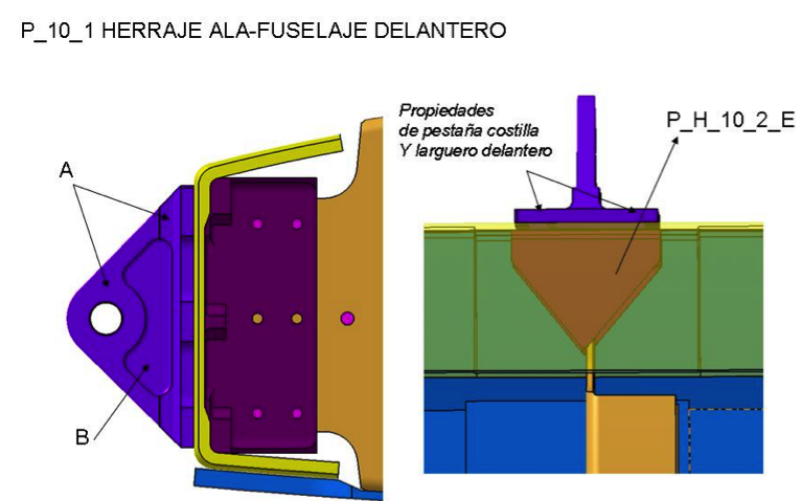

Figura 3-12: Herraje ala-fuselaje delantero (cortesía INTA) 
- Herrajes delanteros. Se restringe desplazamiento en el plano del herraje (ejes X, Z absolutos) en un nodo central. Ver figura 3-14.

Estos nodos centrales situados en el eje del bulón están unidos a los nodos del herraje mediante RBE2.

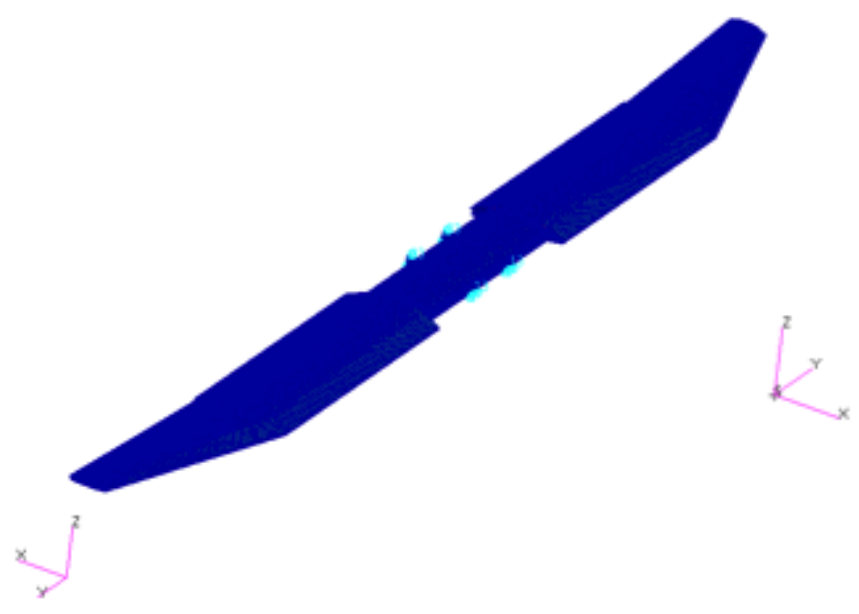

Figura 3-13: Condiciones de contorno del modelo FEM (cortesía INTA)

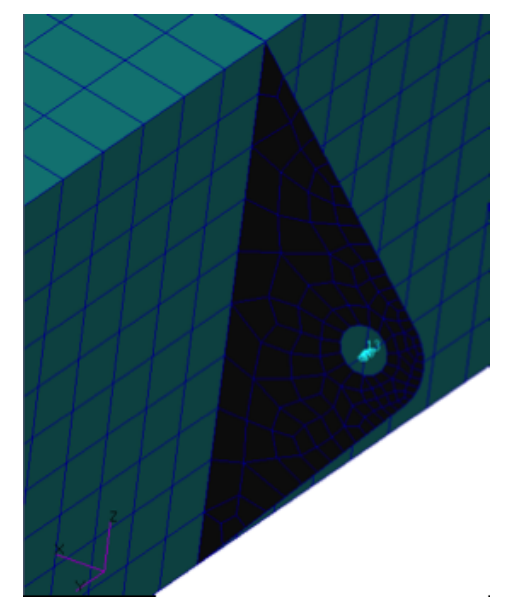

Figura 3-14: Condición contorno herraje delantero (cortesía INTA) 


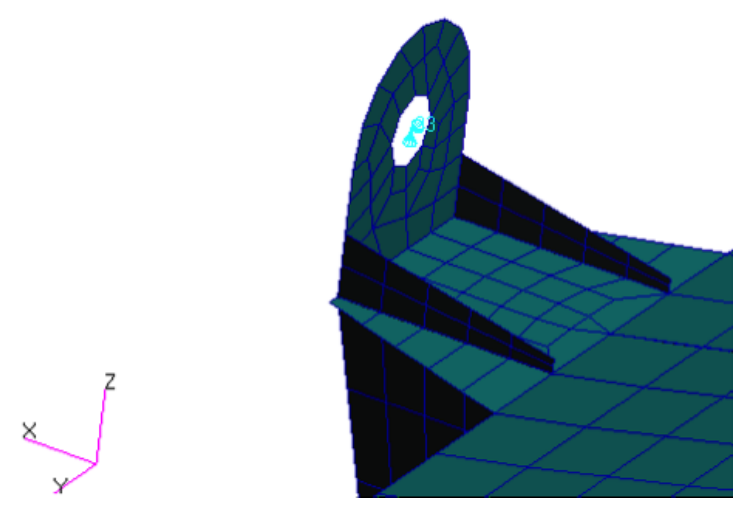

Figura 3-15: Condición contorno herraje trasero (cortesía INTA)

\subsection{Uniones}

Las distintas uniones se han simulado mediante MPC's (multipoint constraint). A continuación se explica las consideraciones tenidas en cuenta en cada una de ellas.

Unión ala central - ala exterior

- Zona trasera Y-: El ala exterior en la zona trasera termina en dos orejetas. Estas orejetas están unidas a un nodo central mediante una RBE3 en todos los grados de libertad salvo las RZ del nodo dependiente, la superior a un nodo central superior y la inferior a un nodo central inferior. Asimismo el ala central acaba en dos orejetas. Estas orejetas están unidas a un nodo central mediante una RBE2 en todos los grados de libertad salvo las RZ de los nodos dependientes, la superior a un nodo central superior y la inferior a un nodo central inferior. Los nodos centrales superiores del ala central y el ala exterior se unen mediante una RBAR en todos los grados de libertad. Lo mismo ocurre con los nodos centrales inferiores. Finalmente uno de los nodos centrales superiores se une a uno de los nodos centrales inferiores mediante una serie de elementos viga que simulan el bulón de la unión.

- Z Zona trasera Y+: Se une de manera análoga.

- Zona delantera Y-: Se une de manera análoga.

- Zona delantera Y+: Se une de manera análoga. 


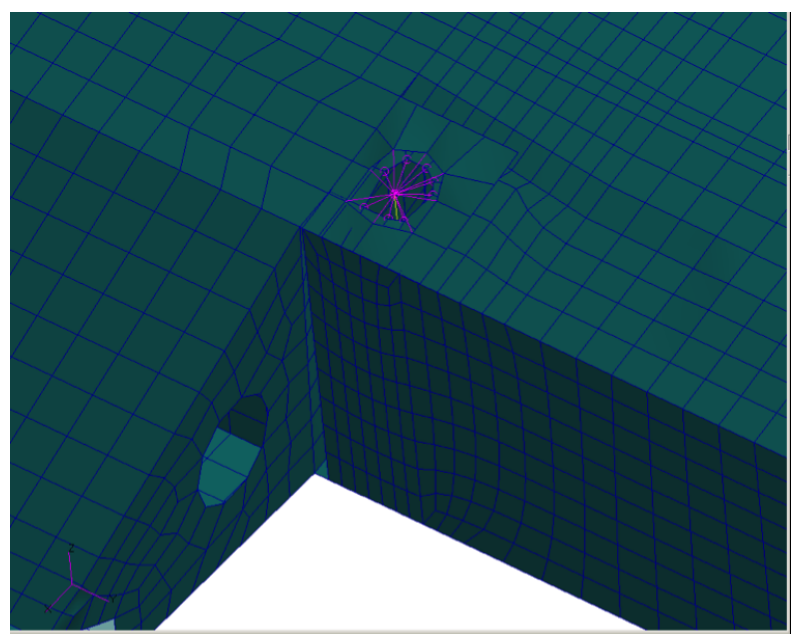

Figura 3-16: Unión ala central-ala exterior (cortesía INTA)

Unión ala central - fuselaje

Los herrajes posteriores terminan en una orejeta y mediante una RBE2 se une a un nodo central en donde se aplican las condiciones de contorno descritas en el apartado anterior.

Los herrajes delanteros a su vez también terminan en una orejeta y también mediante una RBE2 se une a un nodo central en donde se aplican las condiciones de contorno.

\subsection{Descripción del caso de carga}

En todo diseño de aeronave, como es el caso del vehículo aéreo no tripulado considerado, al diseñar la estructura se deben tener en cuenta las cargas que soportará durante su vida operativa. Cada parte del avión estará afectada por diferentes tipos de excitaciones y deben determinarse cuáles pueden ser críticas para su diseño. Hay cientos de casos de carga que pueden clasificarse en cargas en tierra, como las producidas en despegue, aterrizaje, carreteo, etc. y cargas en vuelo como las producidas en las diferentes condiciones de vuelo (velocidad y nivel de vuelo, ciclos de presurización de cabina en su caso, magnitud de la carga de pago y su distribución, etc.) incluidas las maniobras y las causadas por agentes externos como las ráfagas de viento.

En este último caso, como en otros en los que el perfil alar atraviesa el fluido se producen ciertas distribuciones de presión en su superficie que pueden generar cargas en ella como sus- 
tentación, resistencia o momentos relacionados. En el proceso de certificación de una aeronave se considera que debe soportar ciertos tipos de ráfagas de considerable intensidad en función del tipo de aeronave y las misiones que vaya a desempeñar. Es sabido que las causas de la turbulencias que provocan las ráfagas, que son principalmente las tormentas y en menor medida los cúmulos y otros fenómenos atmosféricos como convecciones entre montañas y similares, pueden ocasionar en función de su naturaleza ráfagas discretas, entendidas como fenómenos individuales o aislados, y ráfagas continuas que permanecen en el tiempo [EASA, 2007]. En cualquiera de los casos, este tipo de carga se puede considerar que tiene carácter dinámico. Desde el punto de vista espacial, las ráfagas se consideran verticales, laterales y frontales y en función de ello producen alteraciones en la velocidad relativa del avión, su ángulo de ataque o la sustentación entre otras, debiéndose sumar sus efectos a los producidos por el régimen de vuelo en el que sucede la ráfaga.

Para el caso de estudio, se propone un caso de carga que consiste en la sustentación producida en el ala debida a una ráfaga sinusoidal de viento (ver [Pratt \& Walker, 1954], [Lusebrink \& Brinkspalink, 1994] y [Fung, 1993]) con una velocidad de ráfaga vertical dada por la expresión:

$$
w(x, t)=w_{0} e^{i w(t-x / U)}
$$

Esta ecuación que refleja una ráfaga de forma sinusoidal atravesando la superficie sustentadora con una velocidad U. La sustentación inducida en un perfil bidimensional por unidad de envergadura es:

$$
L=2 \pi \rho b U w_{0} e^{i w t} \phi(k)
$$

donde $\rho$ es la densidad del aire, $b$ es la semicuerda del perfil y $\phi$ depende de la frecuencia reducida $k=\varpi b / U$. La sustentación resultante actúa en el punto $\frac{1}{4}$ de la cuerda del ala con una forma sinusoidal de frecuencia de unos $4 \mathrm{~Hz}$. 


\subsection{Procedimiento de análisis}

Desde un punto de vista práctico, el primer paso para realizar los análisis debe ser la extracción de los datos necesarios del modelo completo de elementos finitos. Se obtienen los siguientes conjuntos de datos:

- $\quad$ El listado de nodos donde se aplica carga y se distribuye a la superficie mojada.

- $\quad$ El listado de nodos con condiciones de contorno.

- $\quad$ El listado de nodos que se unen a los listados anteriores por medio de MPC.

- $\quad$ El listado de nodos donde se mide la respuesta para identificar los parámetros óptimos.

- $\quad$ El listado de nodos que se corresponden con la unión entre punta de ala izquierda y equipo.

- $\quad$ Frecuencias $\boldsymbol{\Omega}$ y autovectores $\boldsymbol{\Phi}$ de los modos propios considerados en todos sus grados de libertad.

Por lo tanto, lo principal es seleccionar los $g$ grados de libertad y $n$ modos de interés para el análisis de forma que sean un grupo reducido $n<g<<G$ de manera que resulten muy manejables incluso para las más grandes estructuras. En el caso propuesto de análisis las dimensiones de las matrices son aproximadamente $G=500000, g=120$ y $n=60$.

A continuación, si es el caso, se pueden añadir los grados de libertad $d$ que la modificación estructural requiera, con lo que se aumentaría la matriz $\mathbf{\Phi}$. Una vez hecho lo anterior el procedimiento es completamente independiente del modelo de elementos finitos y el software que lo maneja con la gran flexibilidad que eso supone.

De acuerdo con las expresiones descritas en el capítulo 2, se pueden construir la nueva matriz de amortiguamiento modificada $\overline{\mathbf{C}}^{*}$ con $\overline{\mathbf{C}}$ y $\overline{\mathbf{C}}^{a}$. Siguiendo también el capítulo 2 , se construyen las matrices $\overline{\mathbf{K}}^{*}$ y $\overline{\mathbf{M}}^{*}$. El paso siguiente es uno de los claves ya que se trata de calcular los autovalores complejos de $\mathbf{A}$ (llamados $\mathbf{\Psi}$ ) y de $\mathbf{A}^{T}$ (llamados $\boldsymbol{\Gamma}$ ) de forma que se pueda completar la matriz de autovalores de $2 \mathrm{n} \times 2 \mathrm{n}$ formada por $\operatorname{los} \lambda_{i}$.

En función del tipo de respuesta buscada, ya sea permanente o transitoria, se pueden calcular sus coeficientes $\bar{A}_{i m}, \bar{B}_{i m}$, en el caso de respuesta permanente, partiendo de los vectores de influencia $b_{i}=\gamma_{i}^{T} \mathbf{B}$ y de la descomposición espectral discreta de $f(t)$ en $M$ funciones de frecuencia $\varpi_{m}$ y amplitud $a_{m}$.

A continuación, se deben calcular las amplitudes en términos de las coordenadas modales 
originales $\left(\boldsymbol{\Psi} \overline{\mathbf{A}}_{m}\right)^{U}$ y $\left(\boldsymbol{\Psi} \overline{\mathbf{B}}_{m}\right)^{U}$ y posteriormente proyectarlas en los grados de libertad físicos de la estructura $\hat{\mathbf{a}}_{m}=\boldsymbol{\Phi}\left(\boldsymbol{\Psi} \overline{\mathbf{A}}_{m}\right)^{S}$ y $\hat{\mathbf{b}}_{m}=\boldsymbol{\Phi}\left(\boldsymbol{\Psi} \overline{\mathbf{B}}_{m}\right)^{S}$. Con estos datos ya se puede obtener la respuesta, ya sean desplazamientos, velocidades o aceleraciones, para los grados de libertad de interés durante un periodo de tiempo determinado.

\subsection{Descripción del tipo de análisis}

El análisis paramétrico considerado muestra la variación de la respuesta en frecuencia entre 0.5 y $12 \mathrm{~Hz}$ (en escalones de $0.1 \mathrm{~Hz}$ ) cuando se tiene en cuenta la adición de un equipo adicional en punta de ala izquierda.

Esto produce cambios en las matrices de masa, rigidez y amortiguamiento del sistema. Estas variaciones locales se parametrizan de la siguiente manera:

- Masa del equipo incluyendo su anclaje: $3 \mathrm{~kg}$ a $5 \mathrm{~kg}$ en 12 escalones iguales

- Rigidez del anclaje: $9720 \mathrm{~N} / \mathrm{m}$ a $18965 \mathrm{~N} / \mathrm{m}$ en 80 escalones iguales

- Amortiguamiento de la unión: $19.1 \mathrm{Ns} / \mathrm{m}$ a 252.7 Ns/m en 80 escalones iguales

Como se realiza un análisis para cada variación de cada uno de los parámetros se ejecutan un total de 76800 análisis con 116 frecuencias entre 0.5 y $12 \mathrm{~Hz}$.

Se ha considerado un amortiguamiento modal de un $1 \%$ en la estructura no modificada.

El equipo debe cumplir ciertos requisitos: su aceleración debe ser inferior a $5 \mathrm{~g}$ dentro del rango $0.5-12 \mathrm{~Hz}$ de forma que se propone el uso de un TMD (tuned mass damper) con ciertas características de masa, rigidez y amortiguamiento para dicho cumplimiento. Adicionalmente, debe verificarse que el desplazamiento en punta de ala derecha no debe ser superior al desplazamiento de la estructura sin equipo.

Las estaciones de control de las variables se muestran en la figura 3-17 y se emplearán para conocer la respuesta en dichos puntos.

De acuerdo con los apartados anteriores, el procedimiento seguido se esquematiza a continuación (ver figura 3-18). 


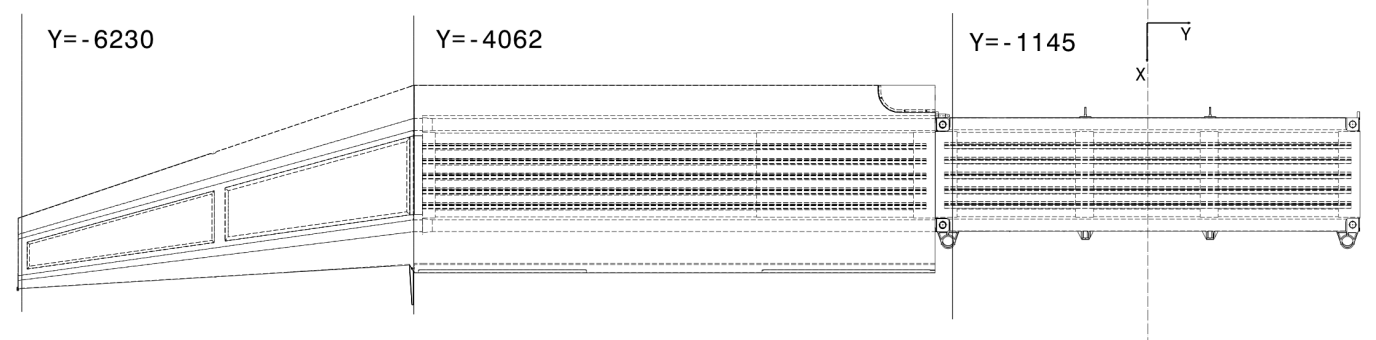

Figura 3-17: Estaciones control en semiala izquierda para análisis por adición equipo en punta ala (cortesía INTA)

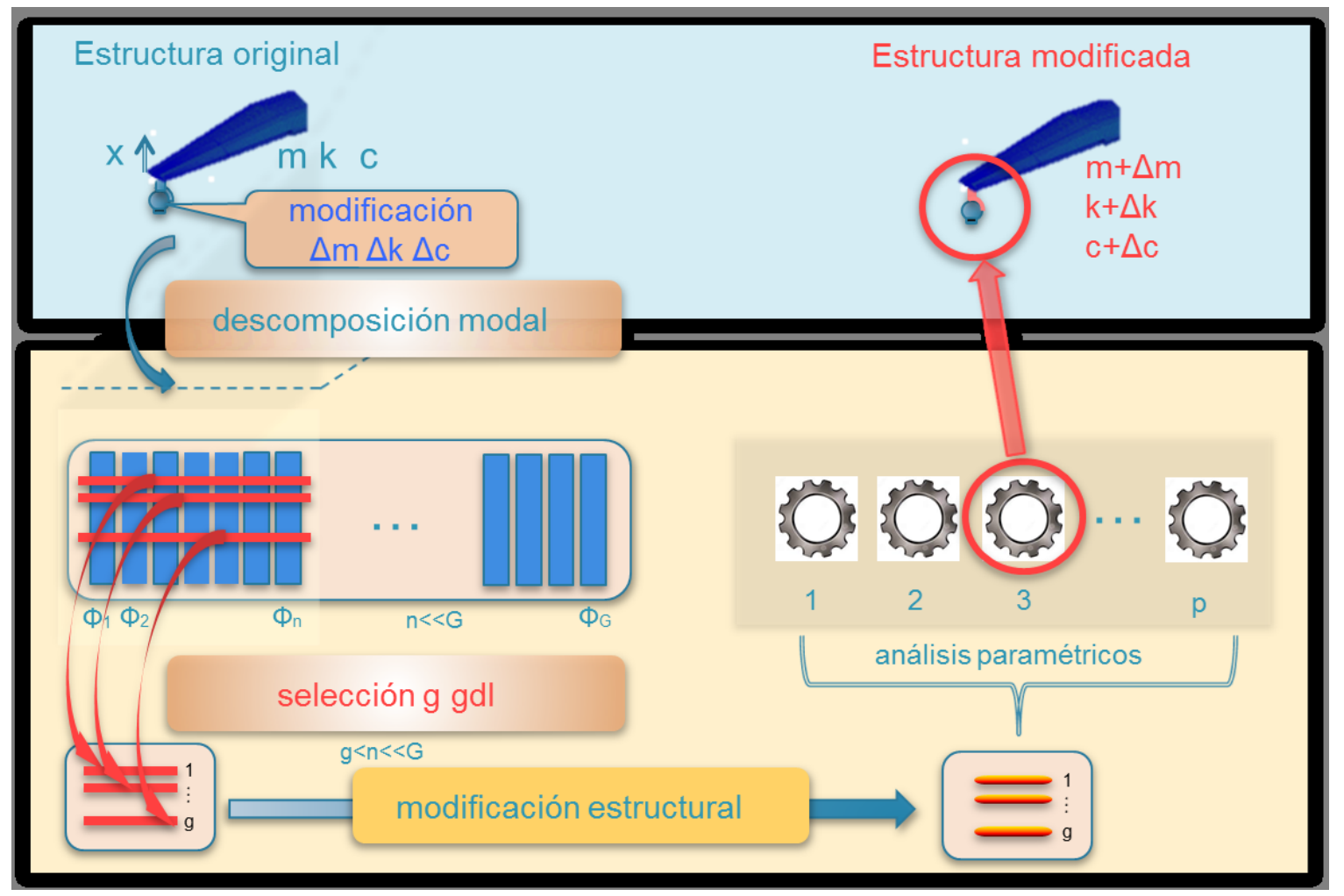

Figura 3-18: Esquema de análisis paramétrico de adición de equipo en punta de ala 
Se parte del modelo de elementos finitos de la estructura original con sus características correspondientes de masa, rigidez y amortiguamiento. Como se ha comentado, en este caso se ha considerado un amortiguamiento modal del 1\%. Determinada por dichas propiedades, la estructura y su modelo proporcionan cierta respuesta frente a la excitación considerada, en este caso la producida por la sustentación generada por la ráfaga en forma de seno.

Como primer paso de todo análisis dinámico se realiza el análisis modal del modelo de elementos finitos para extraer los primeros modos propios reales, sin considerar el amortiguamiento, dentro del rango de frecuencias de interés. Este primer análisis ya proporciona cierto conocimiento al analista pues muestra la disposición de las frecuencias propias y la forma de los modos distribuidos en la estructura. Es importante señalar que para el desarrollo del método solo es necesario el cálculo de los autovalores de un rango de frecuencias determinado por los requisitos, en este caso entre 0.5 y $12 \mathrm{~Hz}$. El rango de frecuencias puede ser cualquiera, no tiene que incluir las frecuencias más bajas como en otros métodos de reducción de modelos. Es recomendable ampliar el cálculo de los autovalores más allá del extremo superior del intervalo por varios motivos. En primer lugar para verificar adecuadamente las variaciones de frecuencia de los modos con las modificaciones estructurales. En segundo lugar porque dependiendo del método de cálculo, la extracción de autovalores es menos precisa en el límite superior del intervalo de cálculo. Por eso se ha ampliado la variación de frecuencias hasta $20 \mathrm{~Hz}$. En cualquier caso, hay que destacar el importante ahorro de recursos de computación que la reducción de la truncatura modal proporciona, tanto en tiempo como en memoria de almacenamiento, . El manejo de los ficheros electrónicos correspondientes a modelos de unos 500.000 grados de libertad tampoco es sencillo, y la extracción de todos los autovalores resulta claramente muy costosa. En el caso de estudio particular se han extraido del modelo de elementos finitos 151 modos correspondientes al rango $0-250 \mathrm{~Hz}$. Como ejemplo, se puede indicar que los tiempos empleados, realizados en un potente servidor de cálculo, en diferentes análisis modales han sido:

- para 21 modos, 135 segundos

- para 151 modos, 420 segundos

- para 638 modos, 810 segundos

Como se puede ver en el esquema 3-18, el paso siguiente es uno de los claves ya que permite 
una segunda reducción del tamaño del problema mediante la selección de los grados de libertad más significativos para el análisis. En el caso de estudio propuesto se han considerado los grados de libertad de los nodos en los que se aplica la carga, los que contienen las condiciones de contorno del modelo y los grados de libertad más adecuados para realizar la monitorización de la respuesta. Estos últimos se corresponden con los nodos del encastre de la semiala izquierda (estación -1145), el punto de inicio del estrechamiento de la semiala izquierda (correspondiente con la estación -4062) y punta de ambas semialas (correspondientes con las estaciones \pm 6230 ), todos ellos en el intradós del larguero delantero. Teniendo en cuenta que se consideran solo los grados de libertad en desplazamientos, los seleccionados son en total 60 grados de libertad. Como puede verse esta reducción puede ser aún más significativa que la correspondiente a la truncatura modal.

A partir de este punto, se aplican las expresiones obtenidas para las modificaciones estructurales propuestas. Como se ha explicado en el Capítulo 2, cada término de las matrices representativos de cada modificación requiere unos cálculos muy limitados ya que cada uno de ellos puede calcularse como la suma de cuatro sumandos. Esto implica que, dado el reducido tamaño del problema, se pueda obtener con facilidad el sistema de ecuaciones dinámico:

$$
\left[\begin{array}{c}
\dot{\mathbf{u}} \\
\dot{\mathbf{v}}
\end{array}\right]=\underbrace{\left[\begin{array}{cc}
\mathbf{0} & \mathbf{I}-\overline{\mathbf{M}}^{a} \\
-\boldsymbol{\Omega}^{2}-\overline{\mathbf{K}}^{a} & -\overline{\mathbf{C}}^{*}
\end{array}\right]}\left[\begin{array}{c}
\mathbf{u} \\
\mathbf{v}
\end{array}\right]+\underbrace{\left[\begin{array}{c}
\mathbf{0} \\
\boldsymbol{\Phi}^{T} \mathbf{P}
\end{array}\right]} f(t)
$$

A

B

que tiene la forma:

$$
\dot{\mathbf{U}}=\mathbf{A} \mathbf{U}+\mathbf{B} f(t)
$$

Que de acuerdo con el procedimiento mostrado en el Capítulo 2, finalmente, mediante álgebra compleja, se puede transformar en un sistema de ecuaciones diferenciales desacopladas en el espacio modal:

$$
\dot{\xi}_{i}-\lambda_{i} \xi_{i}=b_{i} f(t) \quad \text { con } b_{i}=\gamma_{i}^{\mathbf{T}} \mathbf{B}
$$

El reducido tamaño del problema permite prepararlo con las variaciones necesarias de los parámetros que requiera el caso concreto. La aplicación específica desarrollada ha consider- 
ado 12 variaciones de masa del equipo incluyendo su anclaje y 80 tanto en rigidez como en amortiguamiento del conjunto por lo que se han ejecutado en total 76.800 análisis. Se pueden considerar otras variaciones interesantes en función de las especificaciones del problema como:

- aumento del rango de frecuencias de estudio

- reducción del tamaño de alguna de las parametrizaciones, incluida la variación de frecuencia

- modificación de la distribución de las variaciones aumentando la densidad de puntos cerca de algún valor de interés

- parametricación de la amortiguación modal de la estructura

- parametrización de la respuesta en función del número de modos propios considerados

Como se indica en el esquema del análisis paramétrico 3-18, la implementación del método puede permitir la mejor selección del conjunto de parámetros variables en el análisis al programarse los algoritmos de comparación mejores para cada caso. En el propuesto, dado que se trata de la adición de un equipo, aunque por interés académico se haya considerado una variación de la masa, lo más lógico es seleccionar los valores de rigidez y amortiguamiento que mejor cumplen los requisitos. Por lo tanto, de todas las combinaciones de valores que cumplen la requerida reducción de la respuesta en aceleraciones en punta de ala izquierda se selecciona la que ofrece una mayor reducción. Adicionalmente se verifica que se cumple el segundo requisito de desplazamiento de punta de ala derecha.

Es decir, tal y como se refleja en la figura 3-18, se puede transformar la solución, que incluye la participación de la modificación estructural, del espacio modal al espacio físico de forma que queda determinada la mejor combinación de variables del problema. Si se quiere obtener la respuesta de la estructura modificada con la mayor precisión se puede emplear dicha combinación para realizar los análisis que se estimen adecuados sabiendo que el resultado va a ser el mejor de los posibles.

En resumen, se considera un caso de aplicación a una estructura real, con un posible caso de carga y con el que se pretende validar diversos aspectos del método de aceleración de análisis dinámicos: 
- Reducción del modelo por truncatura modal

- Reducción del modelo mediante la selección de grados de libertad significativos del modelo

- Implementación de la adición de nuevos grados de libertad mediante la adición de un nuevo nodo para la simulación del equipo.

- Modificación local de la masa de la estructura mediante la adición de nuevas propiedades de masa

- Modificación local de la rigidez de la estructura mediante la adición de nuevas propiedades de rigidez

- Modificación local del amortiguamiento de la estructura mediante la adición de nuevas propiedades de amortiguamiento

- Realización de diversos análisis paramétricos para la verificación de los requisitos considerados

En apartados posteriores se muestran los diferentes resultados logrados en este proceso.

\subsection{Comportamiento modal}

Como se ha comentado, el primer análisis que se realiza es un análisis modal para la extracción de los autovalores y autovectores en el rango de frecuencias de interés de la estructura sin modificar. En las figuras 3-19, 3-20 y 3-21 se muestran los tres primeros modos propios de la estructura sin el equipo adicional.

Podría describirse el primer modo como un modo de flexión antisimétrico sobre el plano de simetría del UAV mientras que el modo segundo parece que se corresponde con su equivalente simétrico. En cambio el tercer modo parece de rotación en torno al eje Z. En principio, este último modo no entraría dentro del rango de frecuencias de estudio. 


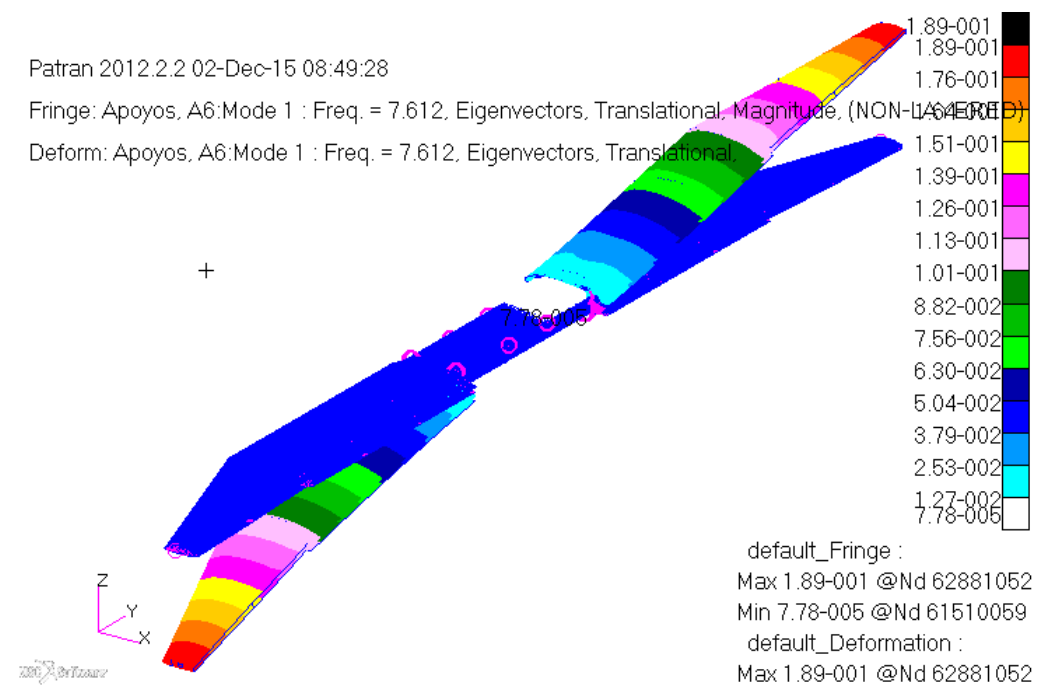

Figura 3-19: Modo 1 de la estructura original: $7.6 \mathrm{~Hz}$

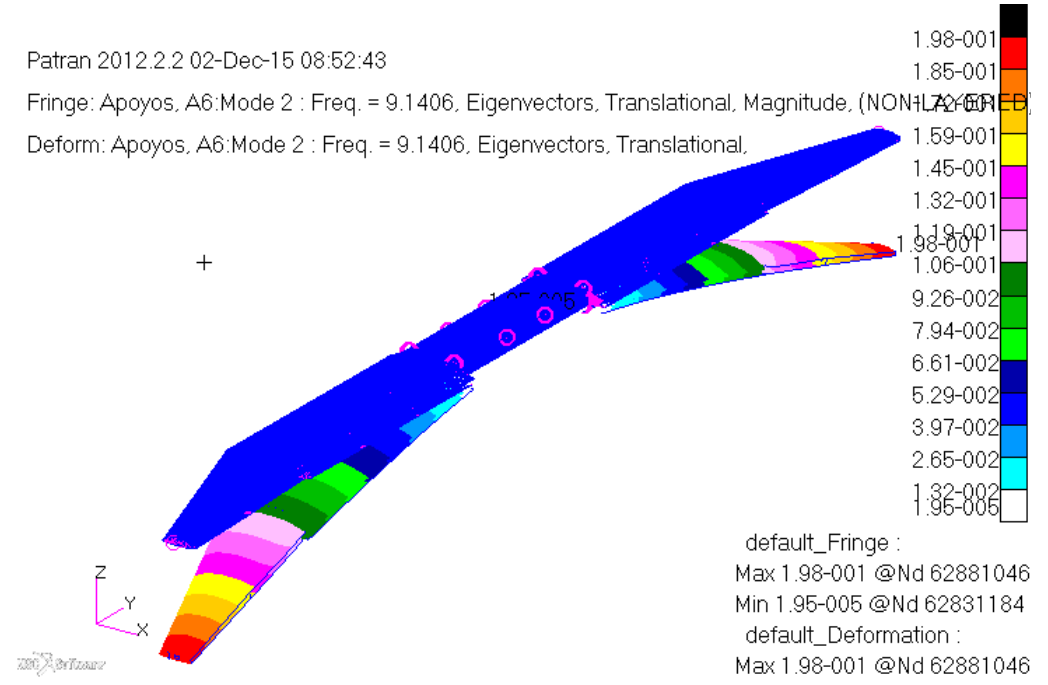

Figura 3-20: Modo 2 de la estructura original: $9.1 \mathrm{~Hz}$ 


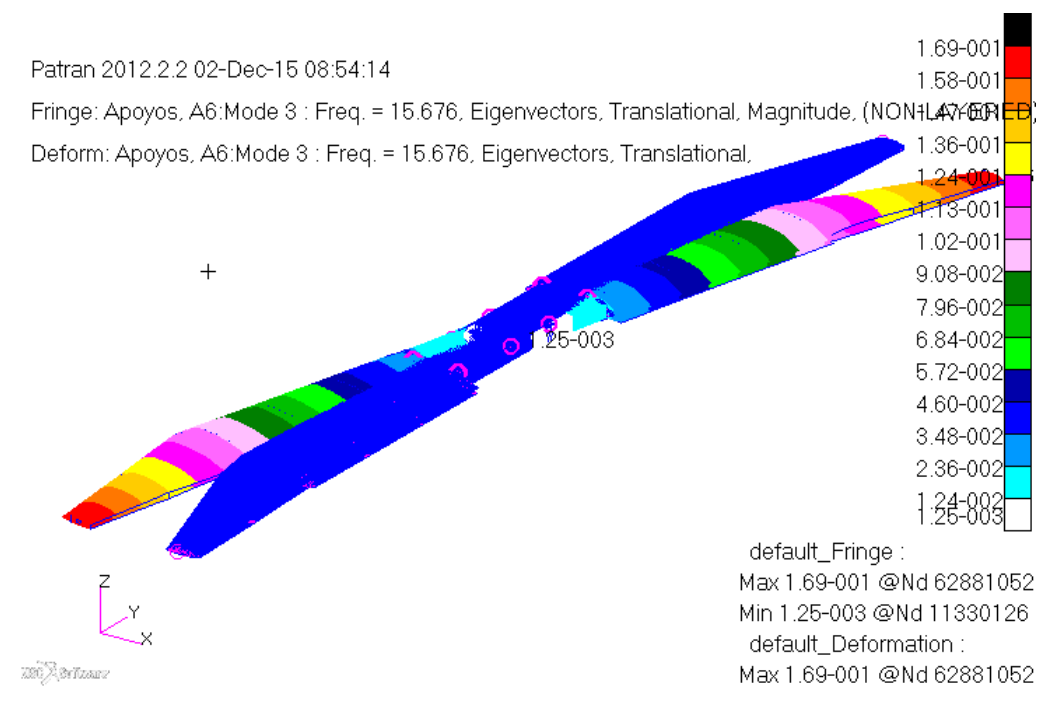

Figura 3-21: Modo 3 de la estructura original: $15.7 \mathrm{~Hz}$ 


\section{Capítulo 4}

\section{RESULTADOS DEL ANÁLISIS PARAMÉTRICO}

Como se ha comentado anteriormente, para verificar el requisito de aceleraciones en punta de ala izquierda se han ejecutado diversos análisis paramétricos. Para representarlos adecuadamente se han recogido en la figura 4-1 una selección de algunas de las respuestas. Las gráficas proporcionan la respuesta en frecuencia de la aceleración vertical de la punta de ala izquierda que es la posición donde se ha añadido el equipo. Cada gráfica está dibujada mediante los análisis realizados en cada una de las 116 frecuencias del rango. Aunque el rango de frecuencias de estudio es entre 0.5 y $10 \mathrm{~Hz}$ se ha representado hasta $12 \mathrm{~Hz}$ para verificar el comportamiento de los modos completamente. La línea roja representa la respuesta de la estructura original, sin la adición del equipo. Puede verse que el resto de curvas ofrecen respuestas cuyos máximos son menores por lo que cualquier caso de los representados proporciona mejor respuesta que la estructura sin modificar. De hecho, se puede observar que la mera adición de masa ya reduce ampliamente la respuesta en términos de aceleraciones en la punta del ala. En la misma figura 4-1 se representan tres curvas de respuesta de la estructura modificada solo con la adición de tres valores distintos de la masa del equipo. También se representan, como muestra de los 76.800 análisis realizados, cuatro combinaciones aleatorias de los parámetros considerados de masa, rigidez y amortiguamiento que puede proporcionar el equipo incluido con su TMD asociado. Puede comprobarse que hay numerosas respuestas que cumplen el requisito de no sobrepasar en 
ningún caso una aceleración de $5 \mathrm{~g}$ (destacada en la figura con una línea de trazos). De todas las posibles respuestas de la estructura, se ha comparado los máximos a las dos frecuencias propias de la estructura modificada dentro del rango de estudio que son aproximadamente 6.1 Hz y 7.6 Hz obteniendose que la combinación de parámetros con los que dichos máximos son menores, y por tanto la respuesta más atenuada, son:

- $\mathrm{Masa}=5 \mathrm{Kg}$

- Rigidez $=13.313 \mathrm{~N} / \mathrm{m}$

- Amortiguamiento= $81 \mathrm{Ns} / \mathrm{m}$

La respuesta correspondiente a esa selección de variables también se identifica en la figura como el caso óptimo.

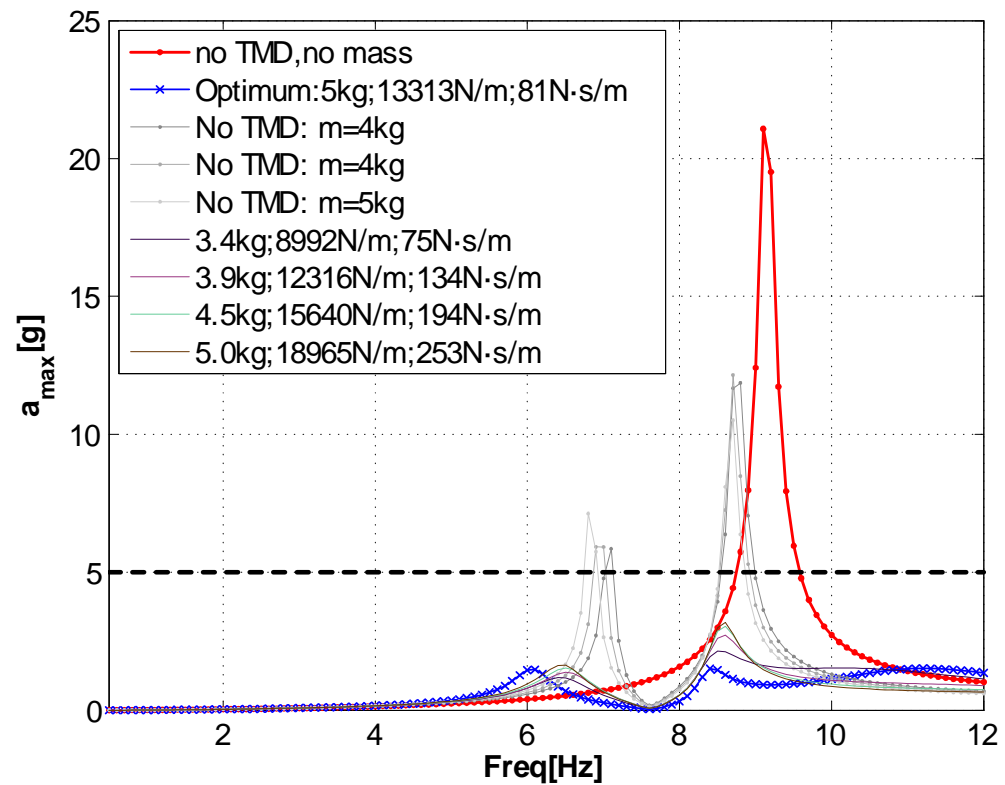

Figura 4-1: Análisis paramétrico. Resumen de respuestas en aceleraciones en punta de ala izquierda

En la figura 4-2 se muestra la respuesta en frecuencia de aceleraciones en la punta izquierda del ala (estación -6230) que puede servir de resumen de lo anterior. En este caso se ha representado, además de la respuesta de la estructura sin modificar, el caso de la adición del equipo 
sin sistema de amortiguación añadido (sin TMD) y el caso óptimo que se corresponde con los parámetros seleccionados según se acaba de explicar.

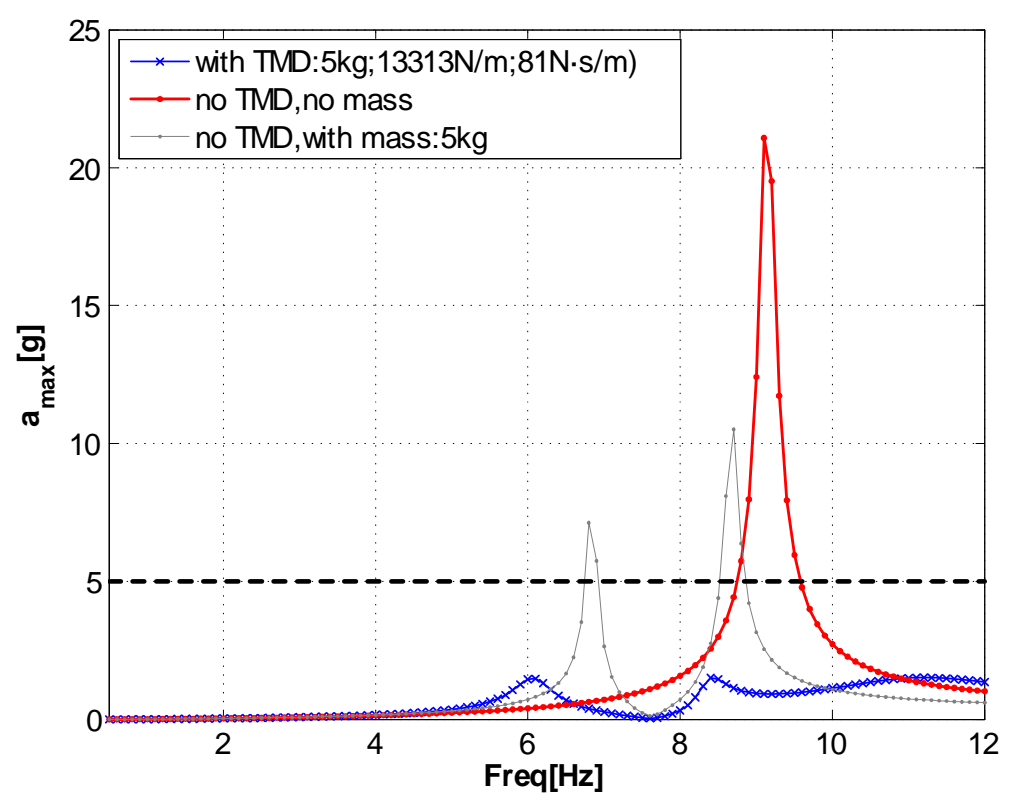

Figura 4-2: Respuesta en frecuencia de aceleraciones en punta de ala izquierda

En la figura 4-3 se muestra la respuesta en frecuencia de aceleraciones en sección del semiala izquierda (estación -4062) donde comienza la zona de estrechamiento que es otro de los puntos de control propuestos en la aplicación. Se ha representado, además de la respuesta de la estructura sin modificar, el caso de la adición del equipo sin sistema de amortiguación añadido (sin TMD) y el caso óptimo que se corresponde con los parámetros seleccionados. Puede observarse que se reduce significativamente la respuesta con las modificaciones propuestas.

En la figura 4-4 se muestra la respuesta en frecuencia de aceleraciones en el encastre del semiala izquierda (estación -1145) que es otro de los puntos de control propuestos en la aplicación. En este caso se ha representado, además de la respuesta de la estructura sin modificar, el caso de la adición del equipo sin sistema de amortiguación añadido (sin TMD) y el caso óptimo que se corresponde con los parámetros seleccionados según se acaba de explicar. Puede observarse que se reduce significativamente la respuesta con las modificaciones propuestas. 


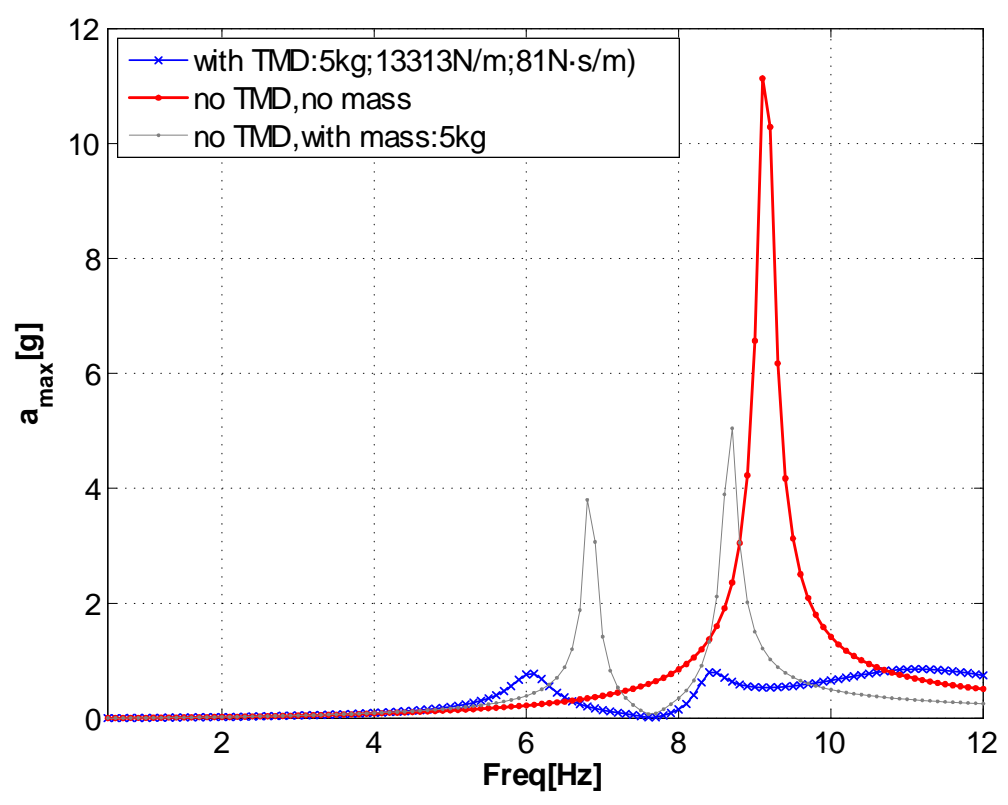

Figura 4-3: Respuesta en frecuencia de aceleraciones en inicio estrechamiento de semiala izquierda

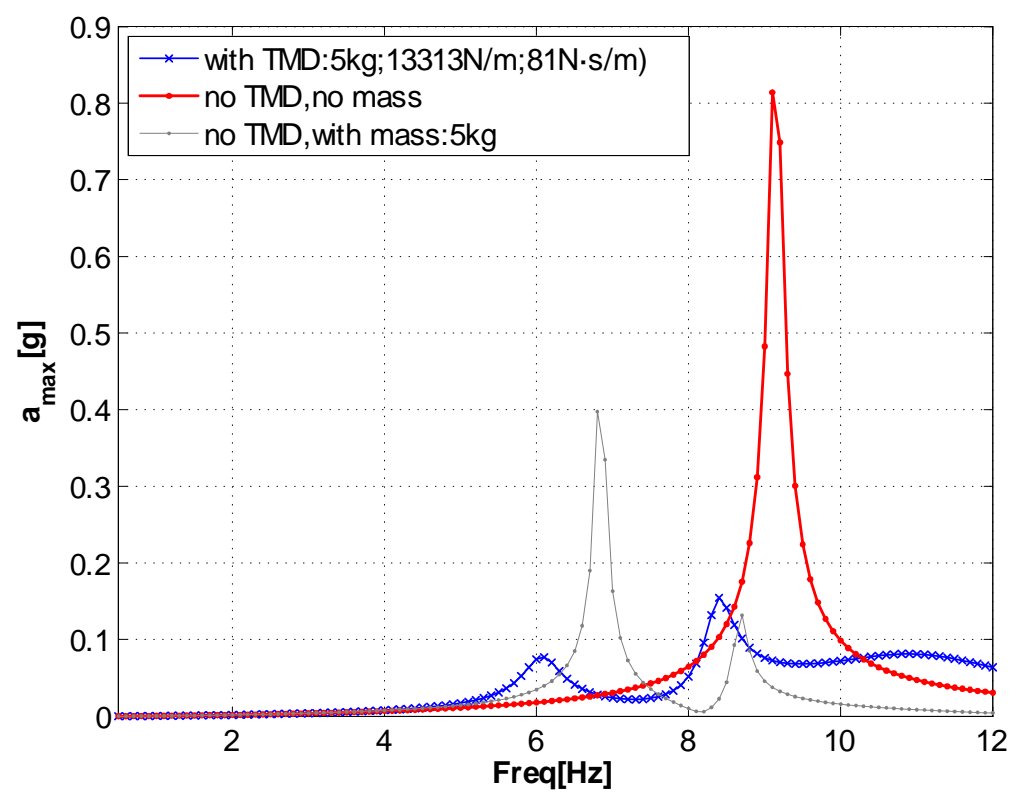

Figura 4-4: Respuesta en frecuencia de aceleraciones en encastre de semiala izquierda 
Como se ha podido observar, varias de las combinaciones de masa, rigidez y amortiguamiento cumplen con los requisitos de aceleración al reducir la respuesta por debajo de $5 \mathrm{~g}$ entre 0.5 Hz y 12 Hz. La combinación óptima de parámetros puede extraerse de los resultados y pueden realizarse con facilidad gran número de análisis demostrando la utilidad del método propuesto. Una vez realizados los análisis, puede adaptarse el tratamiento de los resultados de la forma deseada. Por ejemplo, en la figura 4-5 se puede ver un estudio paramétrico de la variación de la masa en la que se refleja la respuesta de aceleración en la punta de la semiala izquierda en función de la variación de la masa del equipo añadido, considerando la rigidez y amortiguamiento del caso óptimo. Se observa que el aumento de masa reduce la aceleración vertical en la ubicación del equipo añadido, como era de esperar debido al aumento de la inercia.

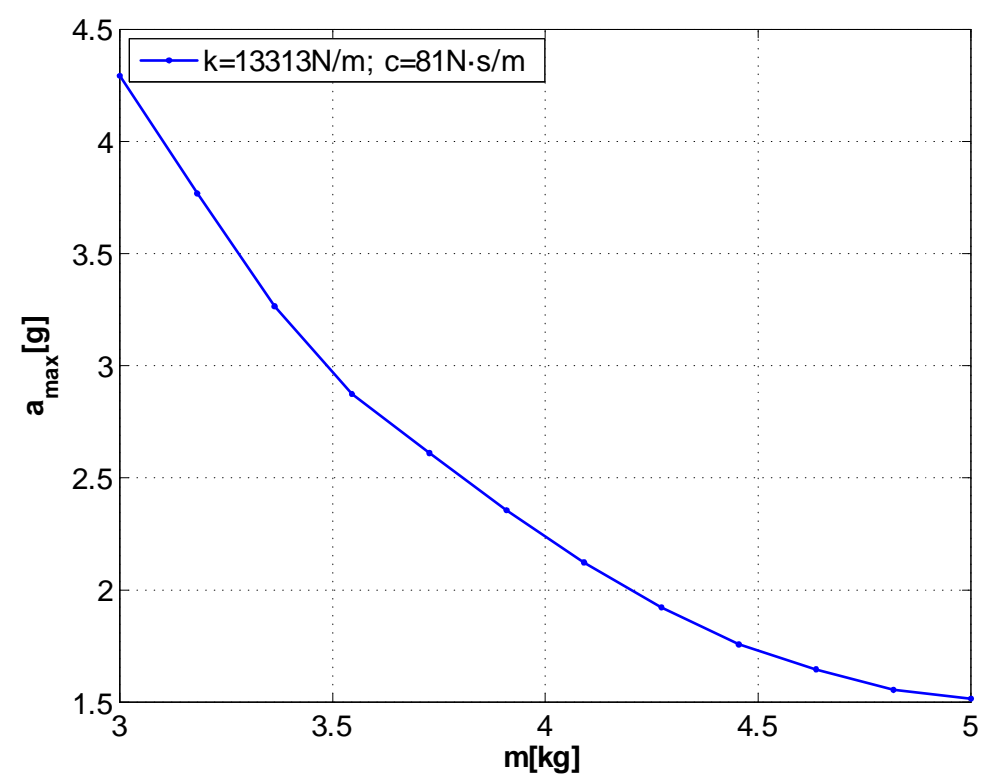

Figura 4-5: Análisis paramétrico en masa

De forma análoga, en la figura 4-7, se puede observar la variación de la respuesta en aceleración en punta de ala izquierda cuando varía el parámetro amortiguamiento y en la figura 4-6 cuando varía la rigidez. Estas gráficas permiten identificar los extremos de los parámetros deseados y las tendencias de las respuestas ante sus variaciones. Esto puede ser interesante ya que, teniendo en cuenta que son válidos rangos de estos parámetros, pudiera ser que por algún motivo, como por ejemplo algún requisito impuesto a posteriori, sea mejor seleccionar un grupo 
de parámetros distinto del óptimo pero con características próximas a él.

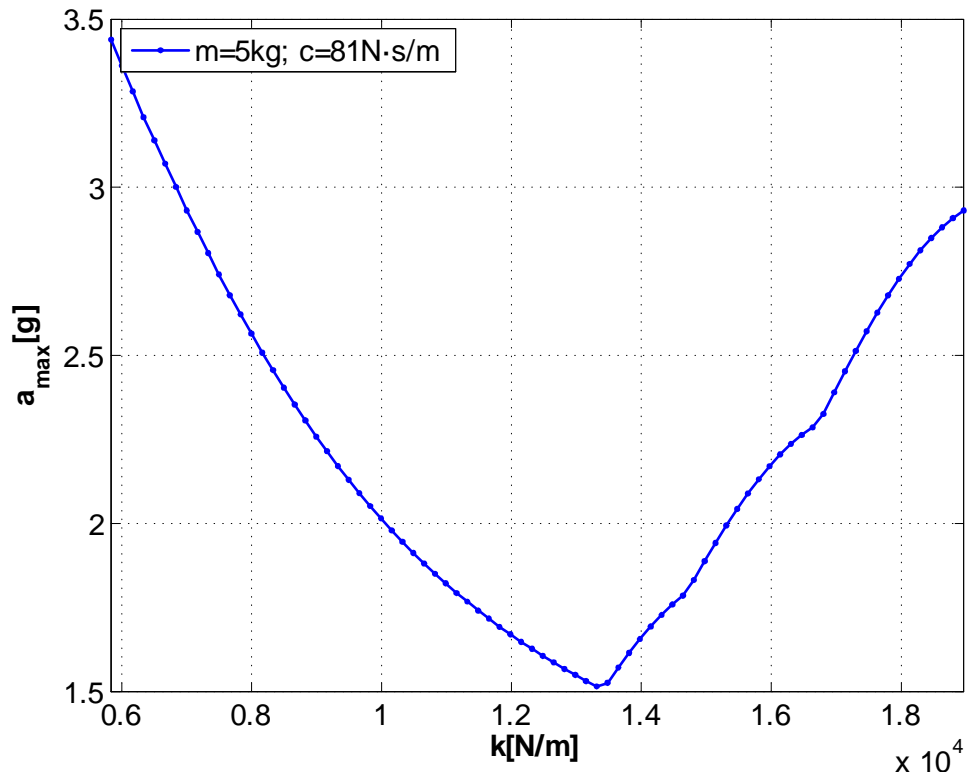

Figura 4-6: Análisis paramétrico en rigidez 


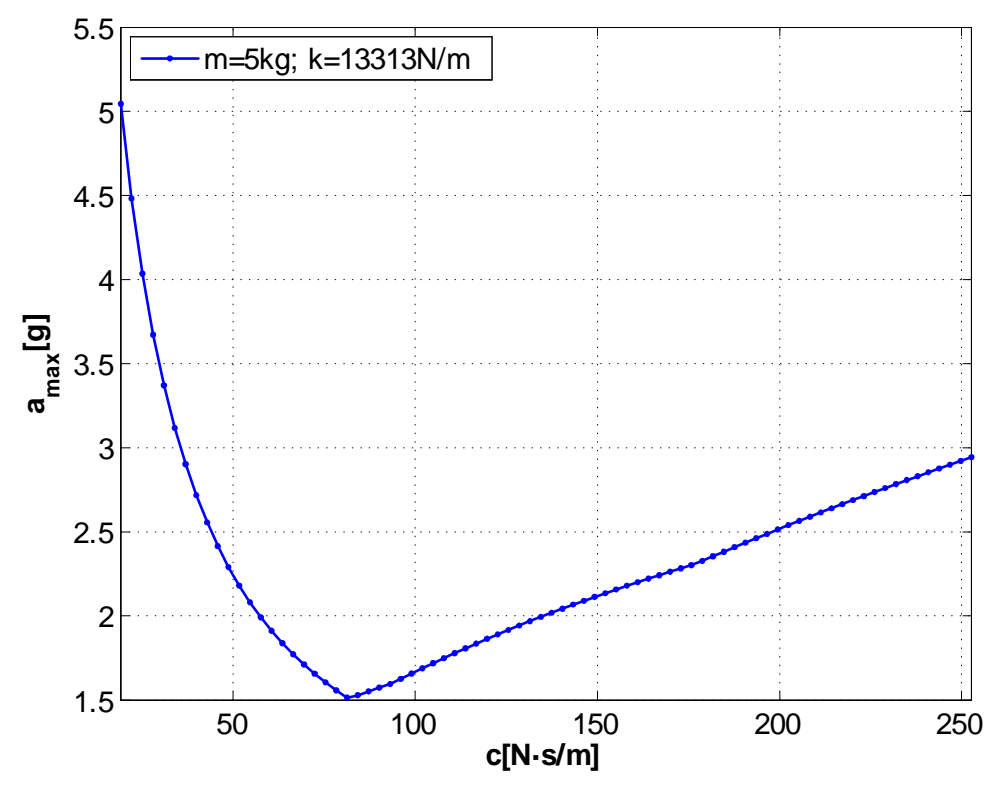

Figura 4-7: Análisis paramétrico en amortiguamiento

Puede comprobarse que ambas curvas presentan un mínimo en la respuesta de aceleración, en el caso del amortiguamiento en $81 \mathrm{Ns} / \mathrm{m}$ y en el caso de la rigidez en $1.33 \cdot 10^{4} \mathrm{~N} / \mathrm{m}$.

En aeronáutica, salvo que sea imprescindible, no se considera el aumento de masa en sistemas de atenuación como TMD sino que es preferible elegir sus propiedades de rigidez y amortiguamiento de forma que se cumplan los requisitos demandados siempre que sea posible. Teniendo esto en cuenta puede construirse una superficie (ver figura 4-8) que relacione la respuesta en aceleraciones de la ubicación deseada con dichas propiedades de forma que el ingeniero pueda identificar cómo interactúan entre ellas, ver las zonas de interés, detectar extremos absolutos y relativos, etc.

La mejor manera para identificar gráficamente los extremos, en el caso de estudio el mínimo de los mínimos de la respuesta de aceleraciones en función de la rigidez y el amortiguamiento, puede ser mediante una gráfica de curvas de nivel como la figura 4-9. En dicha figura se ha marcado la posición y magnitud del máximo y del mínimo de los mínimos. 


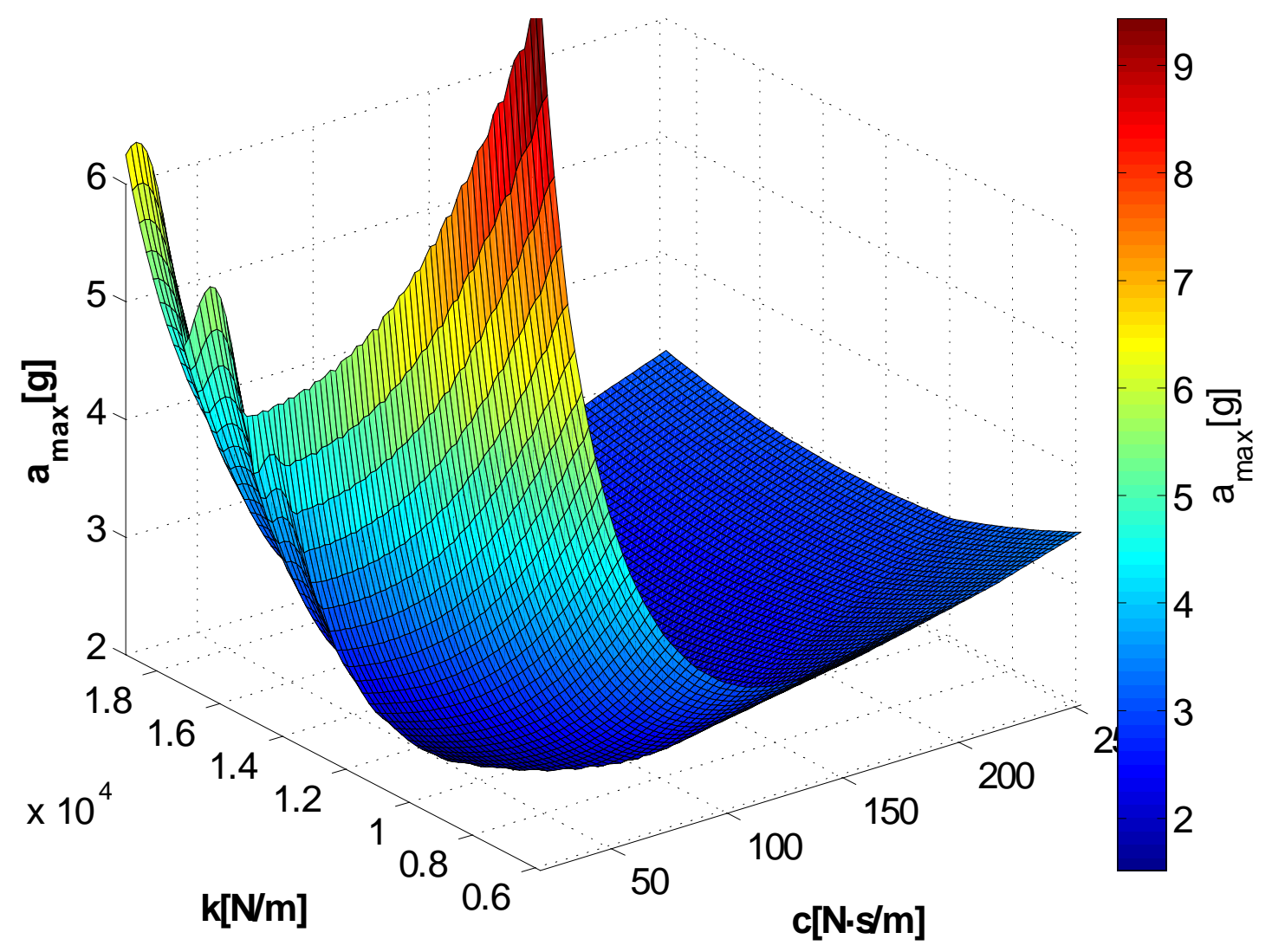

Figura 4-8: Superficie paramétrica rigidez-amortiguamiento 


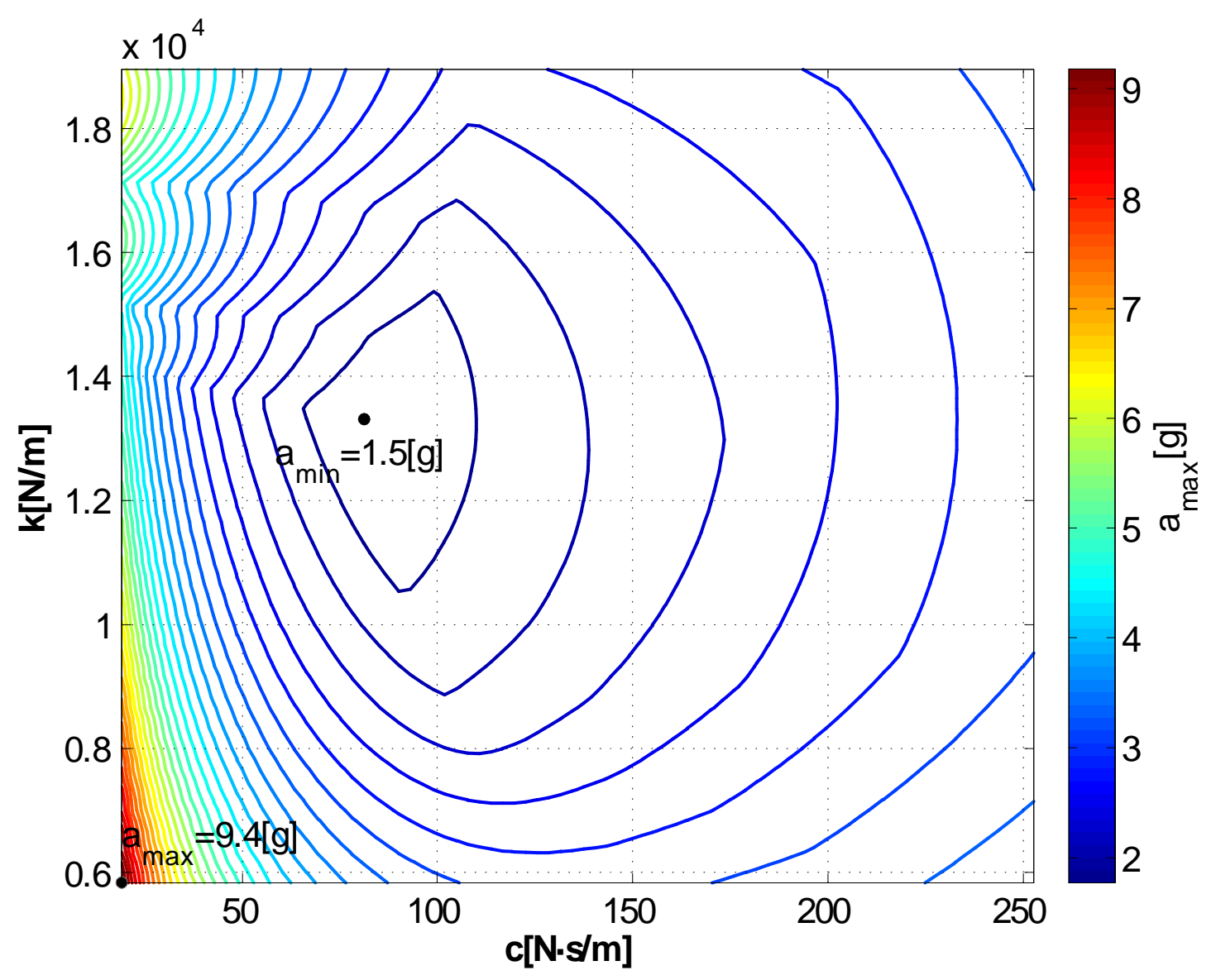

Figura 4-9: Gráfica de líneas isoparamétricas k-c 
El último requisito, que limitaba el desplazamiento vertical en punta de ala derecha se puede igualmente verificar en la gráfica 4-10. Dicha figura representa la respuesta en el rango de frecuencias de estudio del desplazamiento vertical del extremo de la semiala derecha. Como puede observarse el valor del desplazamiento de la estructura con el elemento atenuador (línea azul) ajustado con el conjunto óptimo de parámetros seleccionados es menor que el de la estructura sin modificar (línea roja). Hay que destacar que la adición de un equipo sin TMD no cumpliría con este requisito en desplazamiento, al superar los desplazamientos verticales de punta de ala los valores de la estructura sin modificar.

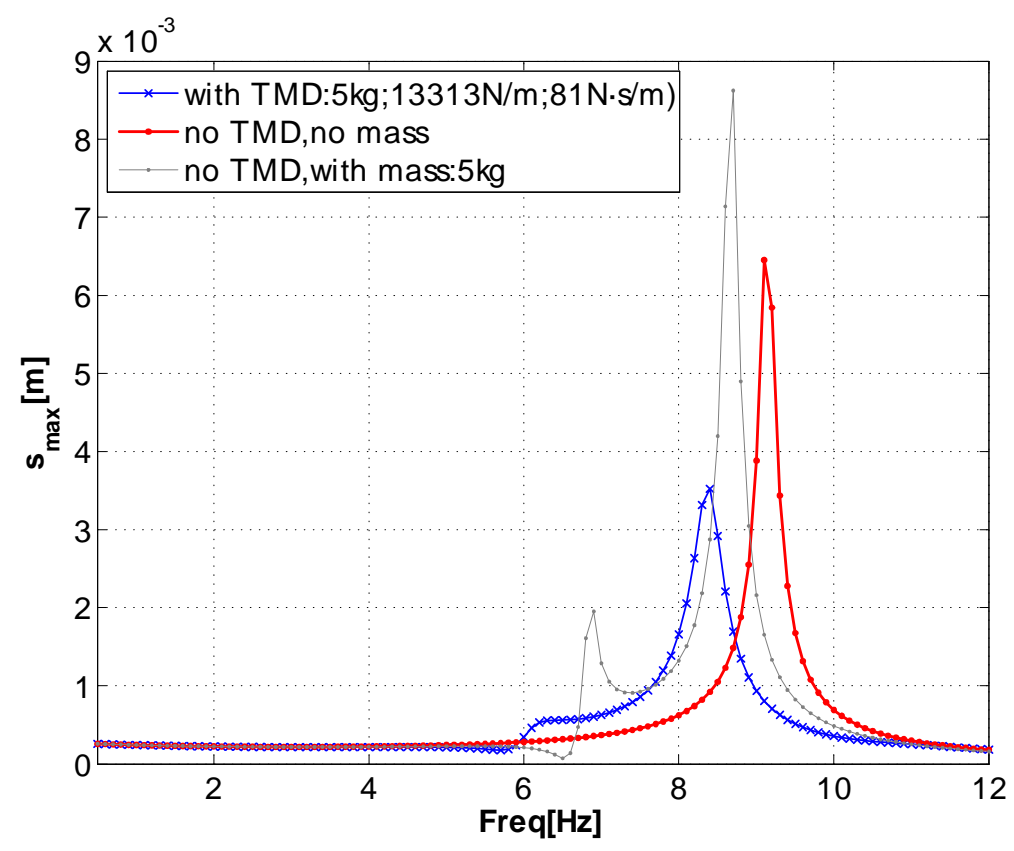

Figura 4-10: Respuesta en frecuencia de desplazamiento en punta de ala derecha

En la figura 4-11 puede verse que la respuesta en desplazamientos en la estación -1145 correspondiente al encastre de la semiala izquierda es menor que los valores máximos de la respuesta sin la modificación estructural, siendo de un valor inferior pero similar en uno de los modos. En cambio, se puede ver en la figura 4-12 que la respuesta en el inicio del estrechamiento es comparativamente menor en ambas frecuencias.

Finalmente, se puede representar la respuesta en frecuencia del desplazamiento de punta de ala izquierda que, de forma similar a la respuesta en aceleraciones se reduce significativamente 


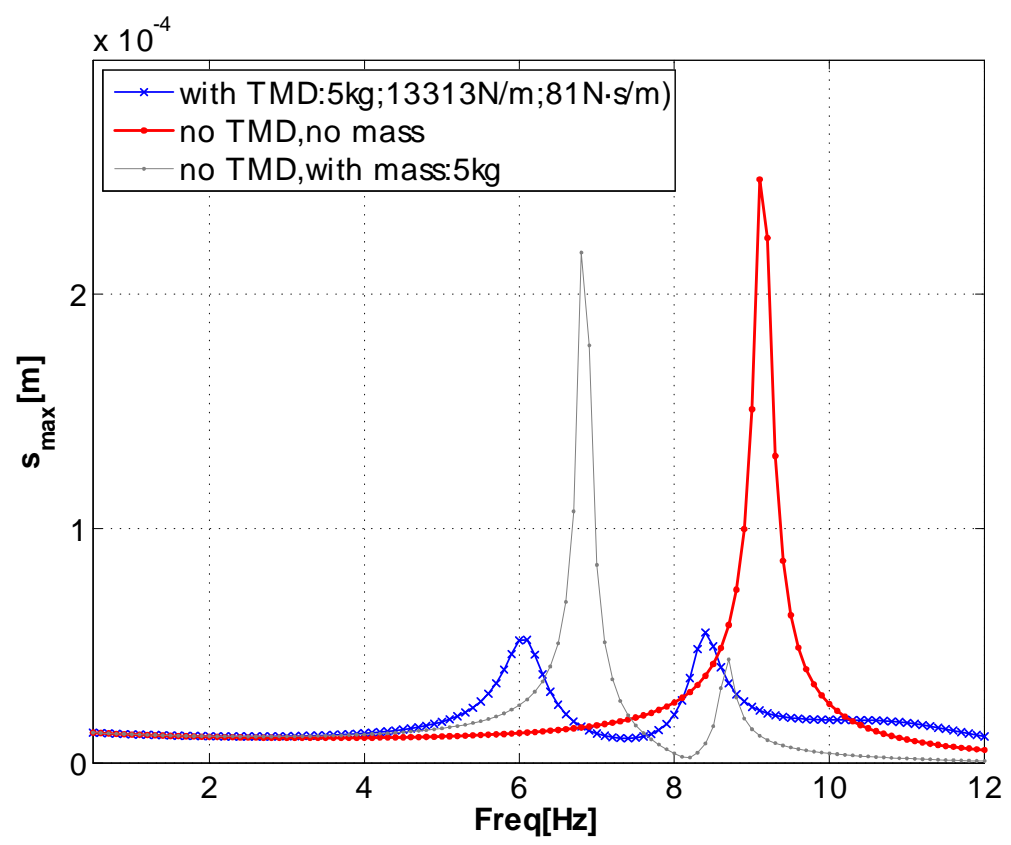

Figura 4-11: Respuesta en frecuencia de desplazamiento en encastre de semiala izquierda

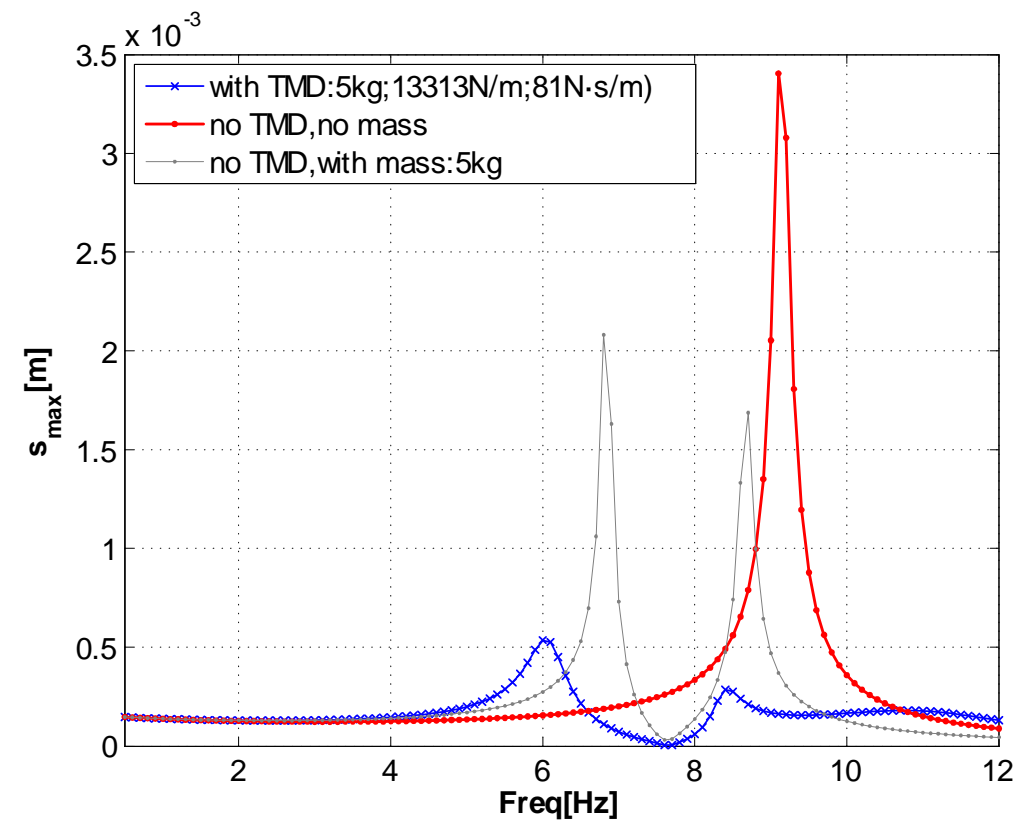

Figura 4-12: Respuesta en frecuencia de desplazamiento en sección inicio estrechamiento de semiala izquierda 
por el empleo del mecanismo atenuador (ver figura 4-13)

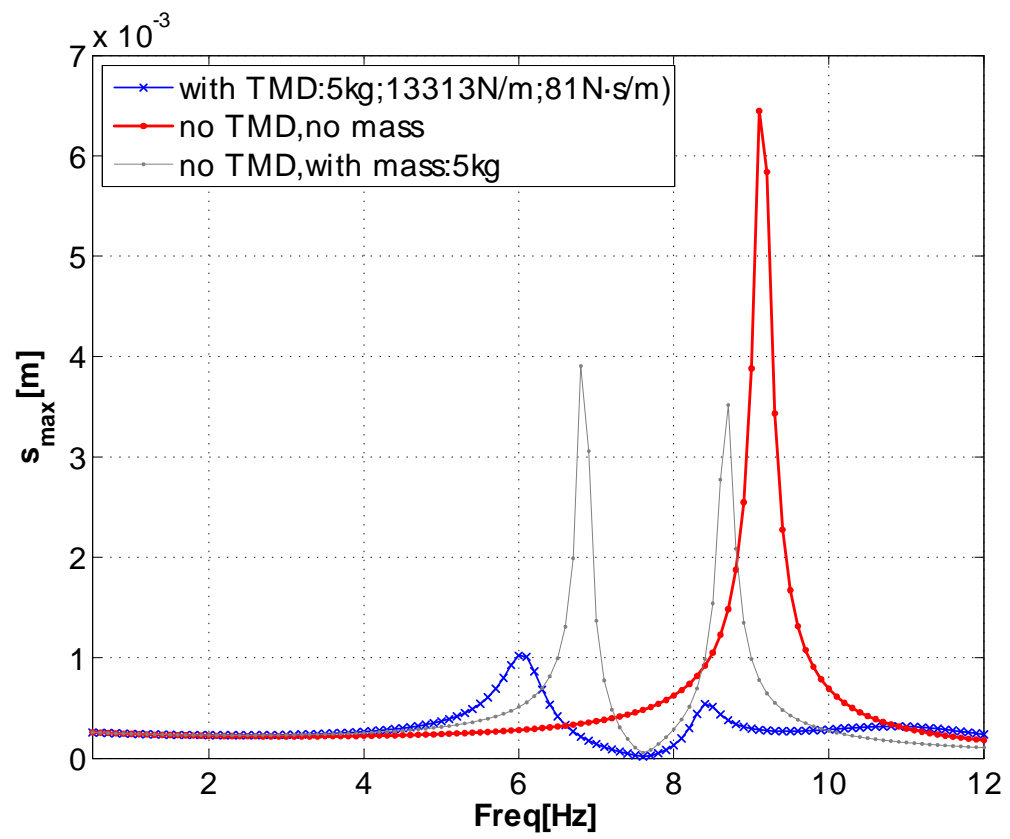

Figura 4-13: Respuesta en frecuencia de desplazamiento de punta de ala izquierda

\subsection{Modificación del comportamiento modal}

Una vez implementada la modificación estructural los modos propios cambian debido a la adición del equipo. Las figuras 4-14,4-15,4-16, 4-17,4-18 y 4-19 muestran los modos propios de la estructura modificada.

Puede verse en las figuras que la presencia del TMD altera sustancialmente los modos y la frecuencia en la que se hallan. 


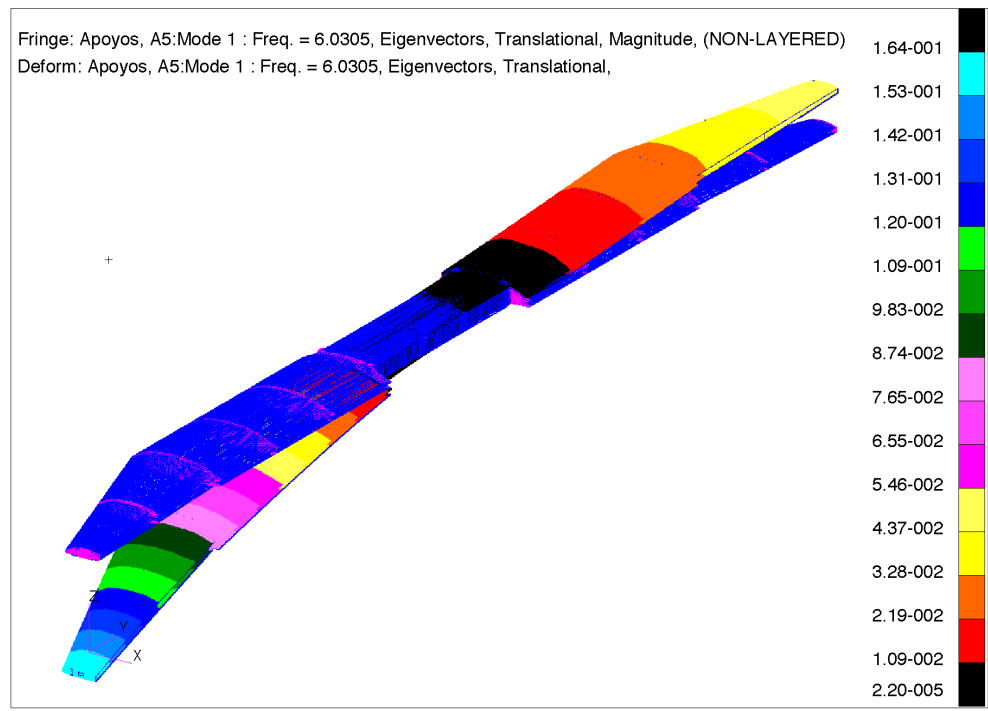

Figura 4-14: Modo 1 de la estructura modificada: $6.0 \mathrm{~Hz}$
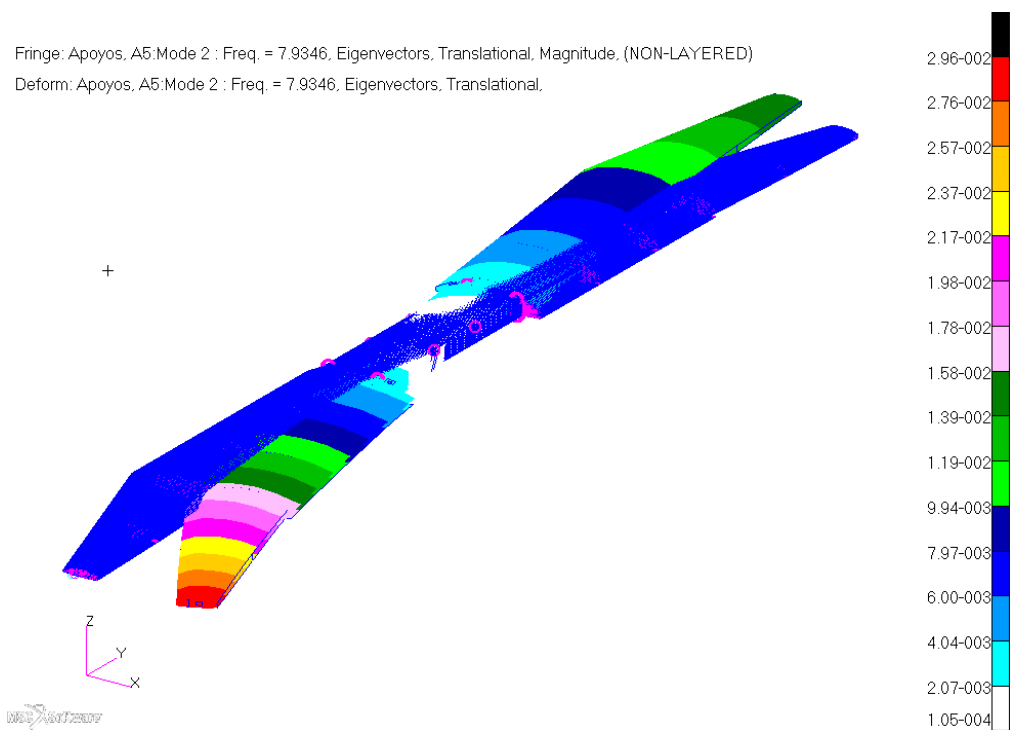

Figura 4-15: Modo 2 de la estructura modificada: $7.9 \mathrm{~Hz}$ 

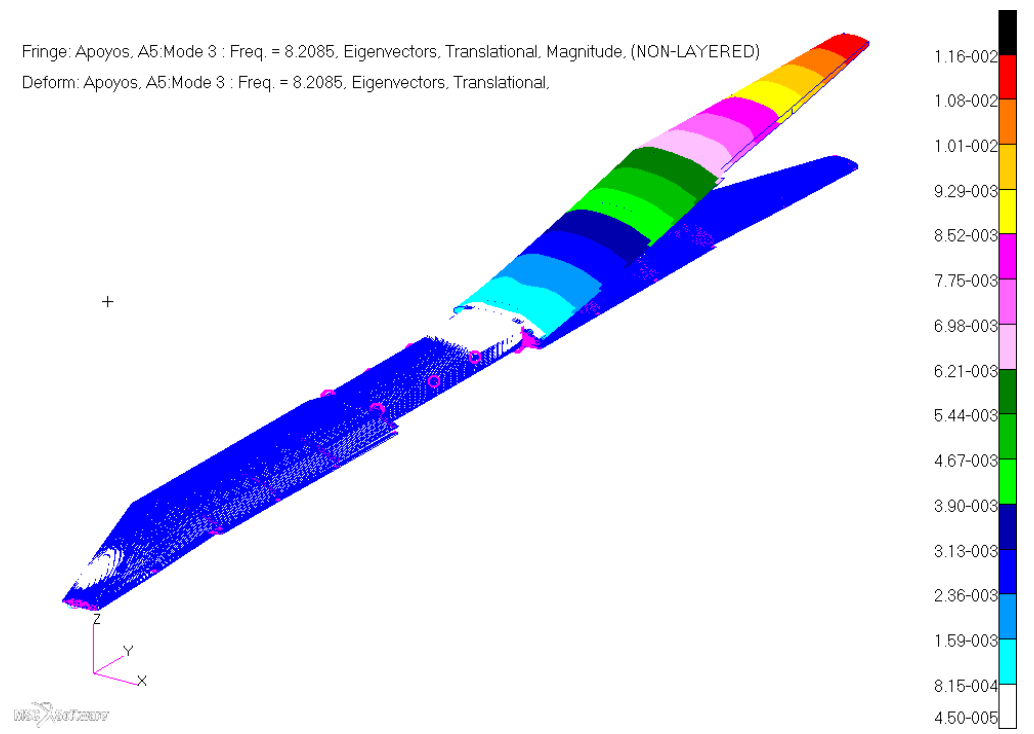

Figura 4-16: Modo 3 de la estructura modificada: $8.2 \mathrm{~Hz}$
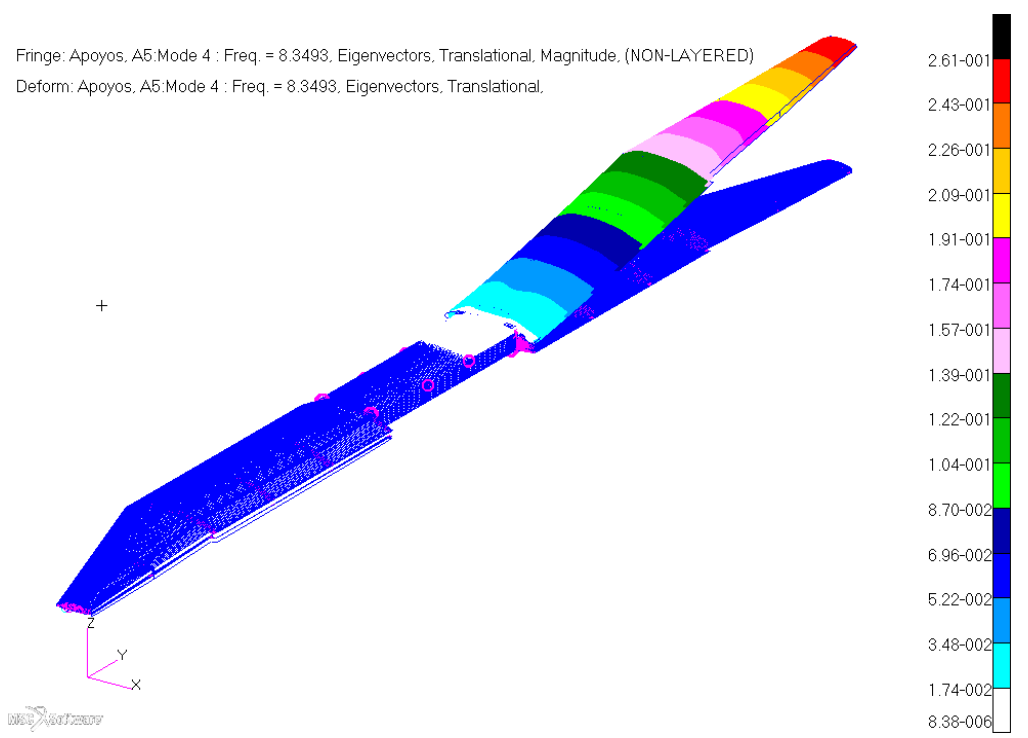

Figura 4-17: Modo 4 de la estructura modificada: $8.3 \mathrm{~Hz}$ 


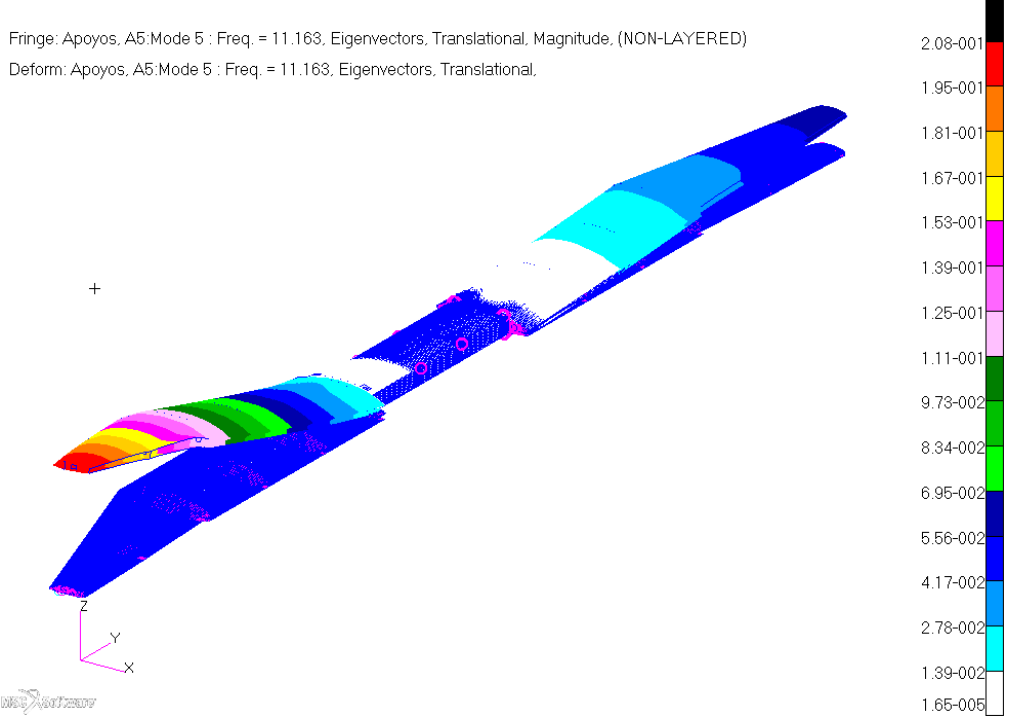

Figura 4-18: Modo 5 de la estructura modificada: $11.2 \mathrm{~Hz}$

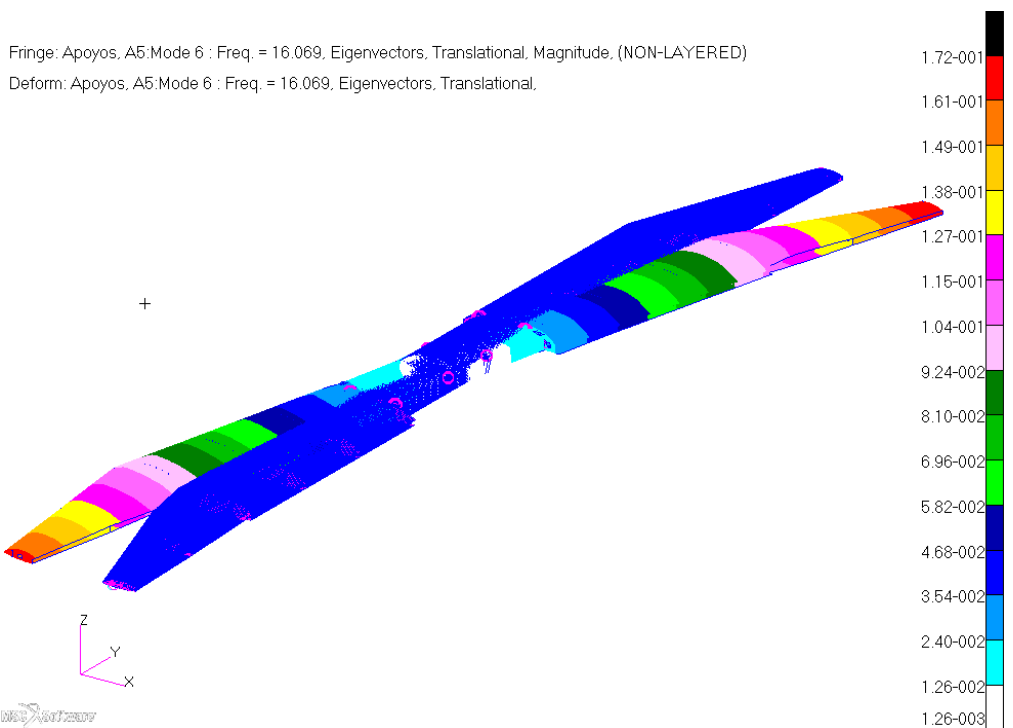

Figura 4-19: Modo 6 de la estructura modificada: $16.1 \mathrm{~Hz}$ 
Para verificar el comportamiento de los modos más allá de las frecuencias propuestas se podría ampliar el rango de estudio. En la figura 4-20 se ha ejecutado un análisis hasta $30 \mathrm{~Hz}$ de forma que se puede representar el comportamiento de la estructura para el conjunto óptimo de valores en este rango ampliado de frecuencia.

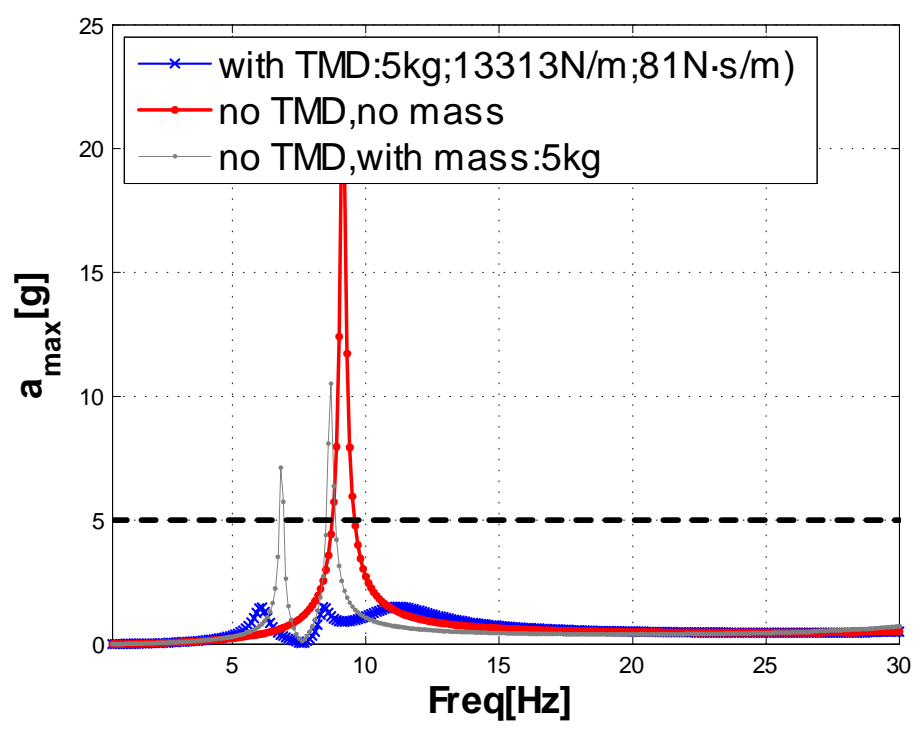

Figura 4-20: Respuesta en frecuencia de aceleraciones en punta de ala izquierda (rango frecuencia $0.5 \mathrm{~Hz}-30 \mathrm{~Hz})$

\subsection{Comparación con los resultados del modelo de elementos finitos}

Para verificar la precisión del procedimiento se han ejecutado diversos análisis del modelo con un programa comercial [MSC.Sofware, 2012] de elementos finitos para el rango de frecuencias de estudio y teniendo en cuenta la combinación óptima de parámetros obtenida: masa de 5kg, rigidez de $13313 \mathrm{~N} / \mathrm{m}$ y amortiguamiento de $81 \mathrm{Ns} / \mathrm{m}$.

En la figura 4-21 se representa la respuesta en frecuencia de la aceleración vertical en punta de ala izquierda para el rango de frecuencias de estudio. De igual manera que para los resultados obtenidos mediante el método de aceleración de análisis, se muestran las curvas correspondientes 
a la estructura sin modificación, los resultados con el equipo añadido como una masa y la respuesta con el equipo añadido y el sistema de amortiguación incorporado y sintonizado con los parámetros óptimos obtenidos. Esta figura se puede comparar con la figura 4-2.

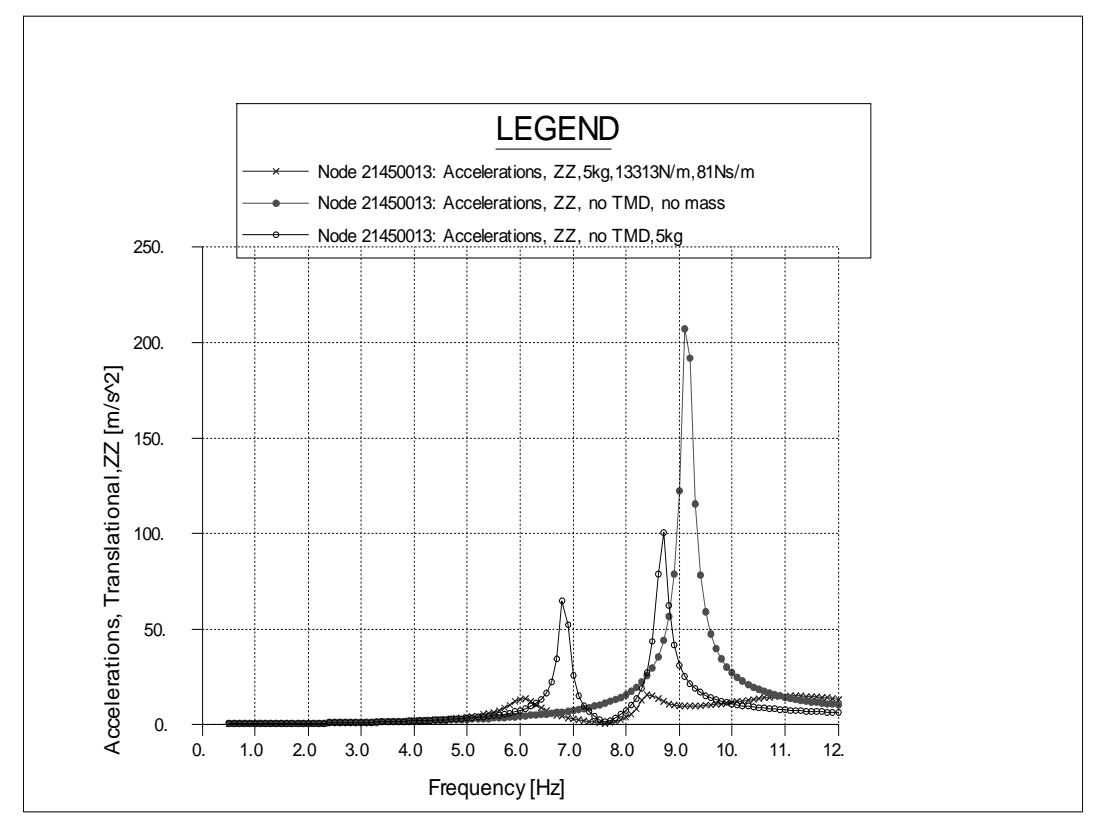

Figura 4-21: Respuesta en frecuencia de aceleraciones en punta de ala del modelo FEM

Se muestra en la tabla 4.1 la comparación de los valores de los picos a $6.8 \mathrm{~Hz}$ y $8.7 \mathrm{~Hz}$ y el valle a 7.6 que permiten identificar la diferencia entre las cifras obtenidas en los análisis paramétricos empleando el método en estudio y los resultados obtenidos mediante el programa comercial de elementos finitos para el caso de adición de equipo sin incluir el TMD.

\begin{tabular}{|c|c|c|c|}
\hline Frecuencia $(\mathrm{Hz})$ & 6.8 & 7.6 & 8.7 \\
\hline Aceleración con método de aceleración $\left(\mathrm{m} / \mathrm{s}^{2}\right)$ & 69.9 & 1.3 & 103.1 \\
\hline Aceleración con programa comercial $\left(\mathrm{m} / \mathrm{s}^{2}\right)$ & 64.3 & 1.2 & 100.4 \\
\hline Diferencia & $9 \%$ & $1 \%$ & $3 \%$ \\
\hline
\end{tabular}

Tabla 4.1: Comparación de máximos de aceleraciones de los diferentes análisis sin TMD

Puede verse que las cifras de respuesta en aceleraciones obtenidas por el método de aceleración estudiado son ligeramente superiores a las producidas por el programa comercial. La 
mayor diferencia se registra en el máximo a $6.8 \mathrm{~Hz}$ que resulta $9 \%$.

En la tabla 4.2 se puede estudiar la comparación de los valores de los picos y valles que permiten identificar la diferencia entre las cifras análogas para el caso de adición de equipo incluyendo el TMD.

\begin{tabular}{|c|c|c|c|}
\hline Frecuencia $(\mathrm{Hz})$ & 6.1 & 7.6 & 8.4 \\
\hline Aceleración con método de aceleración $\left(\mathrm{m} / \mathrm{s}^{2}\right)$ & 14.5 & 0.3 & 14.7 \\
\hline Aceleración con programa comercial $\left(\mathrm{m} / \mathrm{s}^{2}\right)$ & 13.2 & 0.3 & 15.1 \\
\hline Diferencia & $10 \%$ & $3 \%$ & $2 \%$ \\
\hline
\end{tabular}

Tabla 4.2: Comparación de máximos de aceleraciones de los diferentes análisis con TMD

Puede verse en primer lugar que la adición del sistema de atenuación reduce las frecuencias de resonancia de los máximos de aceleraciones ya que la adición del TMD altera no solo los valores de los extremos sino también las frecuencias a las que se encuentran.

En este caso las aceleraciones obtenidas por el método de aceleración de análisis también son similares pero, a diferencia del caso que no incluye el TMD, solo en el máximo a $6.1 \mathrm{~Hz}$ son mayores a los resultados del programa comercial. La mayor diferencia entre los resultados vuelve a encontrarse en el primer máximo, en este caso a $6.1 \mathrm{~Hz}$ y es de un $10 \%$.

Por lo tanto, puede resumirse que las diferencias entre los resultados logrados mediante el método de aceleración de análisis y los obtenidos mediante el programa comercial con realizado sobre modelo de elementos finitos son reducidas en el caso estudiado, sin llegar a superarse el $10 \%$.

Para completar la comparación del método propuesto con los métodos tradicionales de análisis, en segundo lugar se han ejecutado análisis transitorios implícitos Newmark- $\beta$ (MSC.NASTRAN Transient Direct Solution) que proporcionan la respuesta temporal en aceleraciones verticales en el nodo de estudio, en este caso la punta de ala izquierda. Estos análisis no realizan la reducción del modelo por truncatura modal sino que emplean la información de toda la estructura. Se han realizado análisis de este tipo para las frecuencias de los extremos dentro del rango de estudio con un amortiguamiento de un 1\% (ver figuras 4-22, 4-23 y 4-24). 


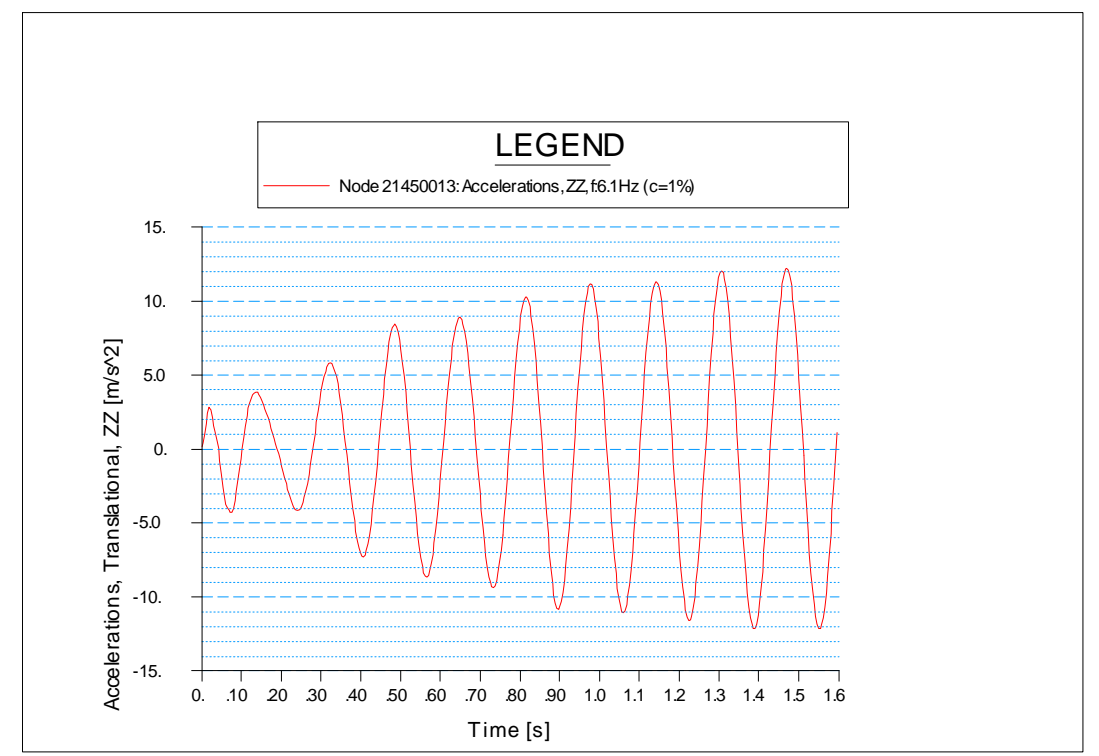

Figura 4-22: Respuesta temporal de aceleraciones en punta de ala izquierda a frecuencia $6.1 \mathrm{~Hz}$

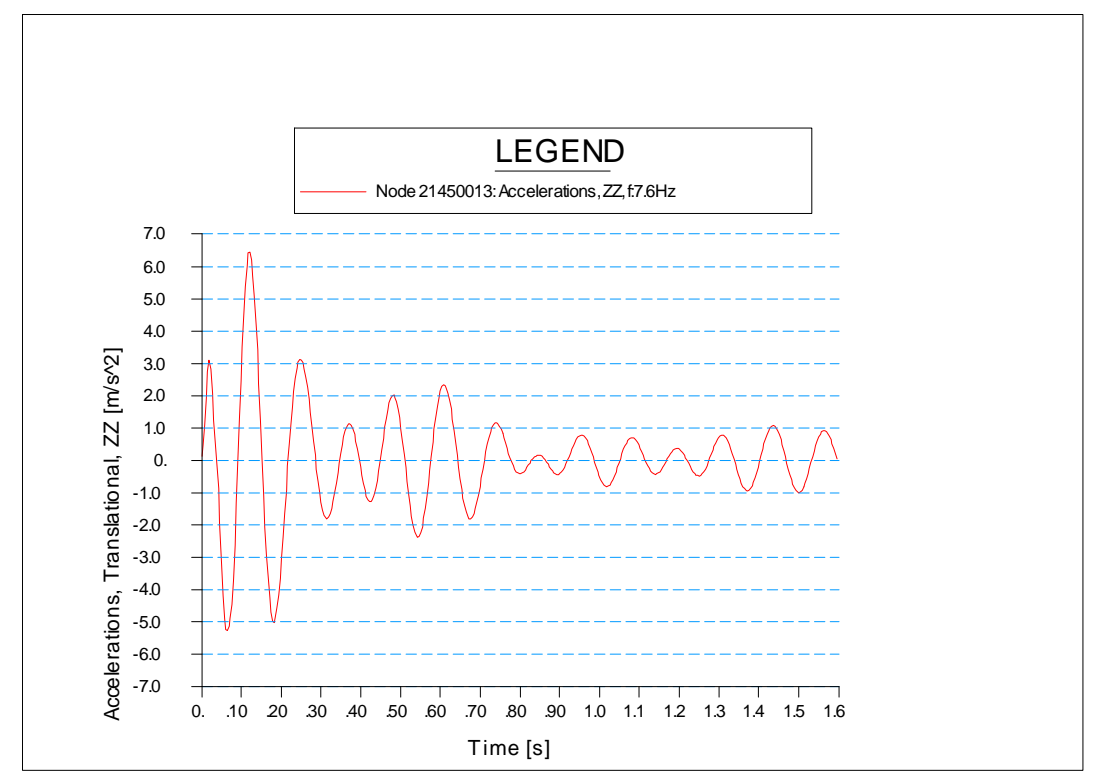

Figura 4-23: Respuesta temporal de aceleraciones en punta de ala izquierda con frecuencia 7.6 $\mathrm{Hz}$ 


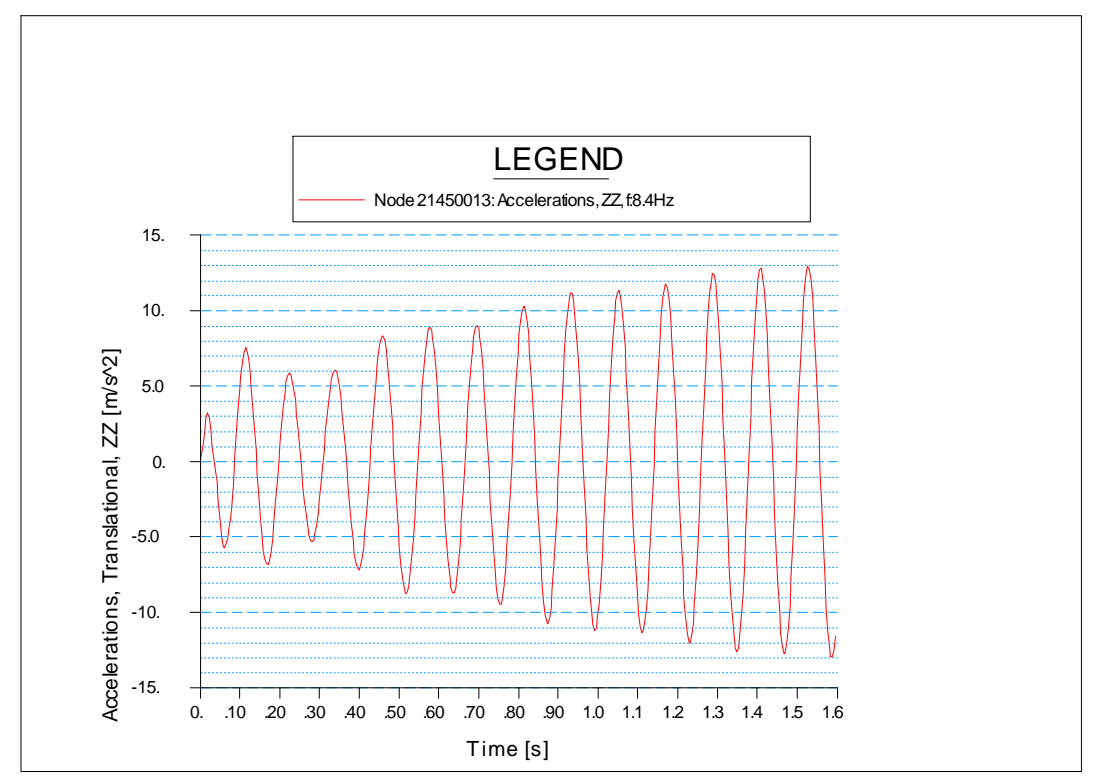

Figura 4-24: Respuesta temporal de aceleraciones en punta de ala izquierda a frecuencia $8.4 \mathrm{~Hz}$

Se observa que las respuestas tardan en estabilizarse debido al pequeño amortiguamiento modal pero finalmente lo hacen en los siguentes valores:

- Figura 4-22 frecuencia 6.1Hz: $12.2 \mathrm{~m} / \mathrm{s}^{2}$.

- Figura 4-23 frecuencia 7.6Hz: $0.9 \mathrm{~m} / \mathrm{s}^{2}$

- Figura 4-24 frecuencia 8.4Hz: $13 \mathrm{~m} / \mathrm{s}^{2}$

Como puede comprobarse en la tabla 4.3, el valor correspondiente a la frecuencia de 6.8 $\mathrm{Hz}$ difiere en un $8 \%$ entre ambos resultados, mientras que el máximo a $8.4 \mathrm{~Hz}$ presenta una desviación de un 16\%. La diferencia en el mínimo es mayor, del orden de un 57\%.

\begin{tabular}{|c|c|c|c|}
\hline Frecuencia $(\mathrm{Hz})$ & 6.8 & 7.6 & 8.4 \\
\hline Aceleración con método de aceleración $\left(\mathrm{m} / \mathrm{s}^{2}\right)$ & 13.2 & 0.4 & 15.1 \\
\hline Aceleración con programa comercial $\left(\mathrm{m} / \mathrm{s}^{2}\right)$ & 12.2 & 0.9 & 13 \\
\hline Diferencia & $8 \%$ & $57 \%$ & $16 \%$ \\
\hline
\end{tabular}

Tabla 4.3: Comparación de máximos de aceleraciones con análisis transitorio 
Vistas las diferencias se plantea aumentar el número de ciclos para incrementar la estabilidad de la respuesta hasta $2.4 \mathrm{~s}$ en vez de $1.6 \mathrm{~s}$. Las gráficas 4-25, 4-26 y 4-27 muestran las respuestas con el intervalo ampliado.

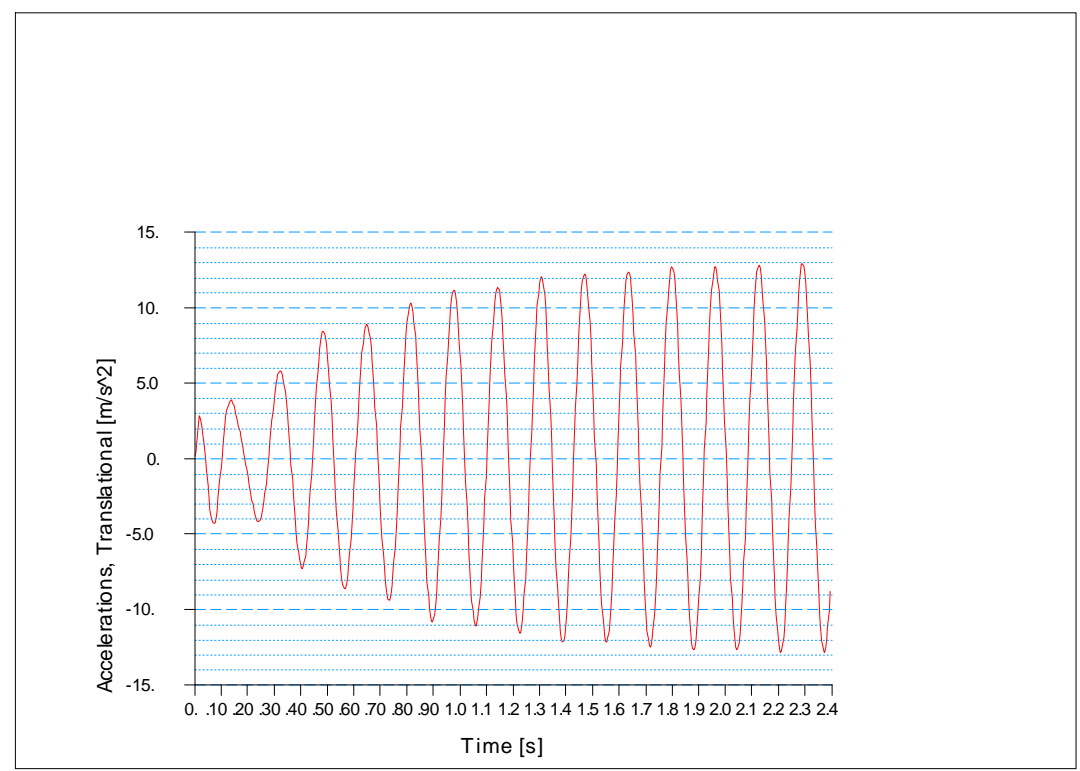

Figura 4-25: Respuesta temporal de aceleraciones en punta de ala izquierda a frecuencia $6.1 \mathrm{~Hz}$ $(2.4 \mathrm{~s})$

Los resultados se recogen en la siguiente tabla:

\begin{tabular}{|c|c|c|c|}
\hline Frecuencia $(\mathrm{Hz})$ & 6.1 & 7.6 & 8.4 \\
\hline Aceleración con método de aceleración $\left(\mathrm{m} / \mathrm{s}^{2}\right)$ & 13.2 & 0.4 & 15.1 \\
\hline Aceleración con programa comercial $\left(\mathrm{m} / \mathrm{s}^{2}\right)$ & 12.8 & 0.4 & 14.3 \\
\hline Diferencia & $3 \%$ & $10 \%$ & $5 \%$ \\
\hline
\end{tabular}

Tabla 4.4: Comparación de máximos de aceleraciones con análisis transitorio ampliado

Como puede comprobarse, el incremento de tiempo en los análisis transitorios disminuye notablemente las diferencias entre los dos métodos empleados, hasta llegar a una máxima diferencia del $10 \%$ en el mínimo localizado a $7.6 \mathrm{~Hz}$ que sigue siendo el valor menos estable. 


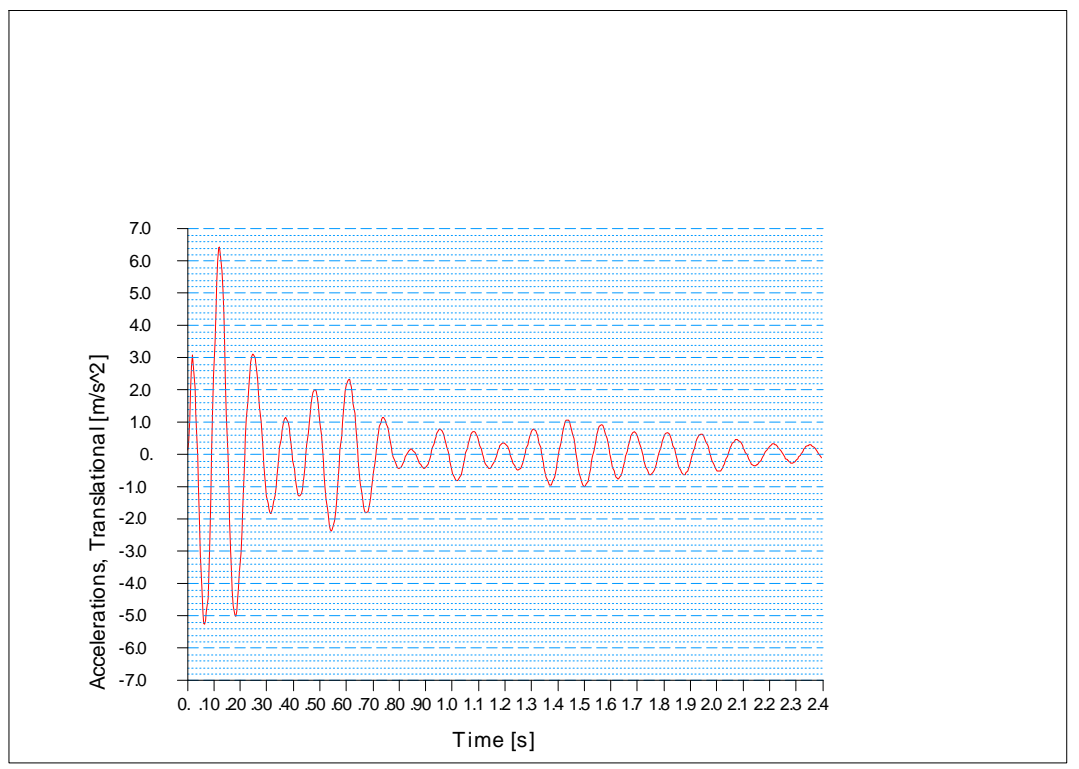

Figura 4-26: Respuesta temporal de aceleraciones en punta de ala izquierda a frecuencia $7.6 \mathrm{~Hz}$ $(2.4 \mathrm{~s})$

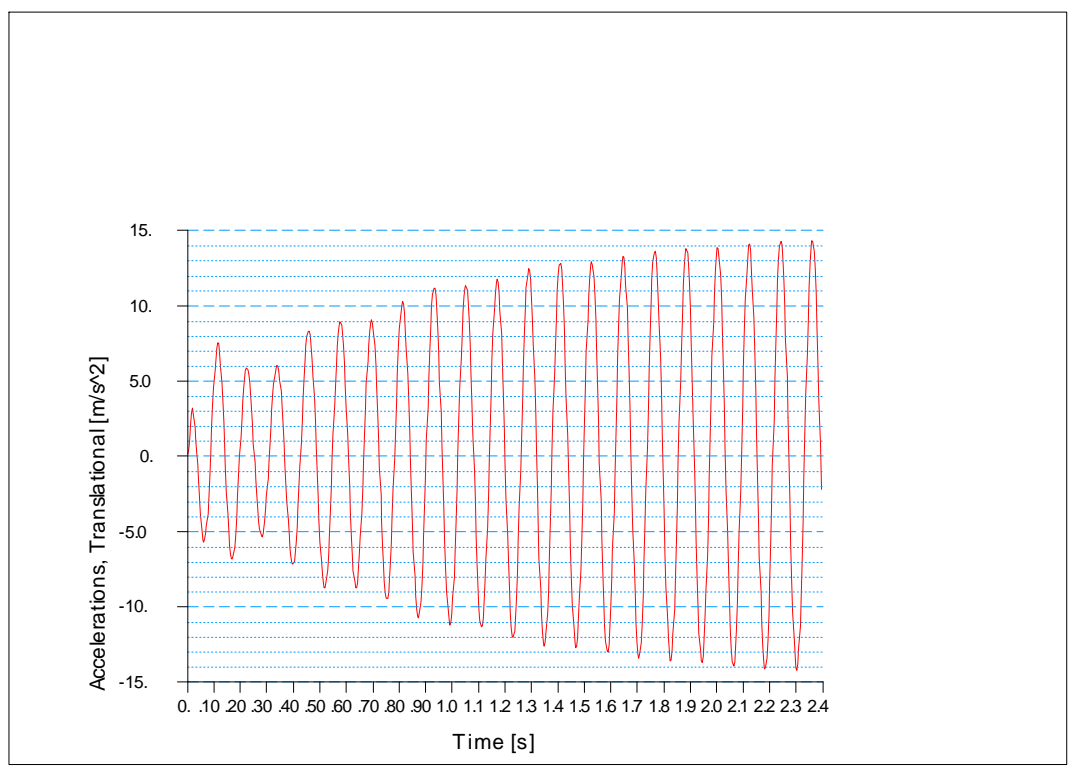

Figura 4-27: Respuesta temporal de aceleraciones en punta de ala izquierda a frecuencia $8.4 \mathrm{~Hz}$ $(2.4 \mathrm{~s})$ 


\subsection{Influencia del amortiguamiento modal de la estructura}

Es particularmente interesante conocer el comportamiento del método de aceleración de análisis paramétricos frente a diferentes valores de amortiguamiento modal considerado en la estructura.

Se han realizado unos análisis paramétricos de respuesta en frecuencia con las siguientes variaciones:

- Masa del equipo incluyendo su anclaje: $3 \mathrm{~kg}$ a $5 \mathrm{~kg}$ en 6 escalones iguales

- Rigidez del anclaje: $9720 \mathrm{~N} / \mathrm{m}$ a $18965 \mathrm{~N} / \mathrm{m}$ en 30 escalones iguales

- Amortiguamiento de la unión: $19.1 \mathrm{Ns} / \mathrm{m}$ a 252.7 Ns/m en 30 escalones iguales

Como se realiza un análisis para cada variación de cada uno de los parámetros se ejecutan un total de 5400 análisis con 116 frecuencias entre 0.5 y $20 \mathrm{~Hz}$. Para identificar las diferencias se ha considerado un amortiguamiento modal de un $5 \%$ en la estructura no modificada en vez de un $1 \%$.

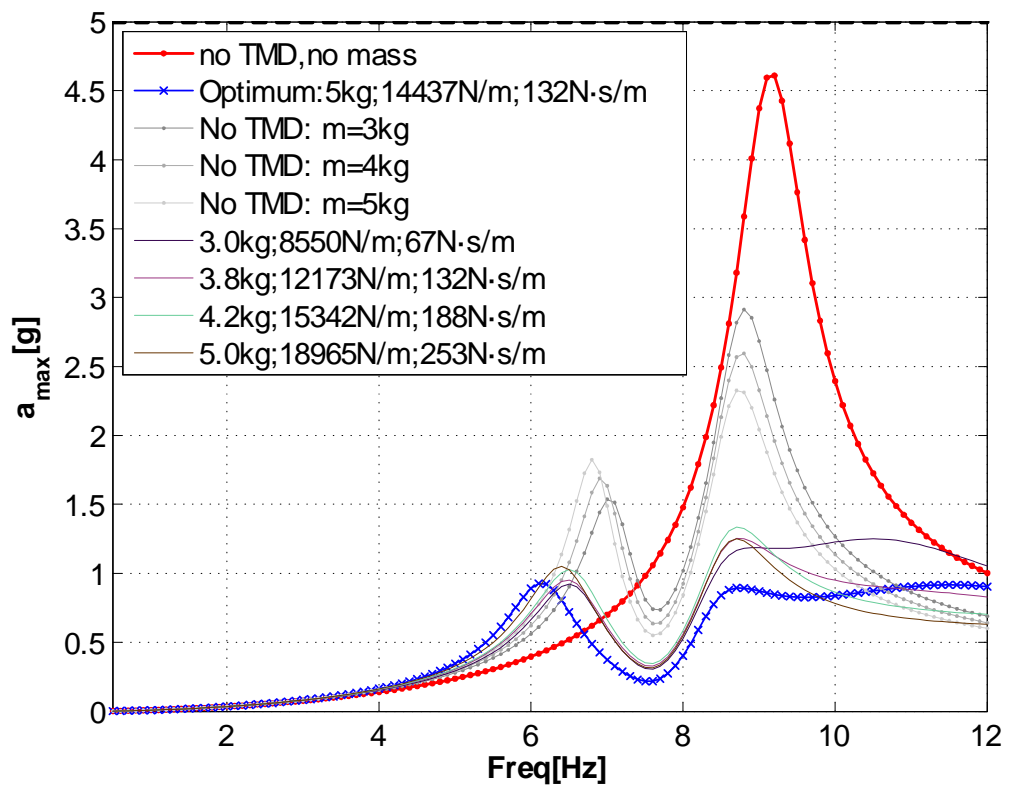

Figura 4-28: Análisis paramétrico. Resumen de respuestas en aceleraciones en punta de ala izquierda (amortiguamiento modal 5\%) 
Se observa que el conjunto de valores óptimos de propiedades del sistema de amortiguación es distinto que para el caso de un 1\%, alcanzándose para $5 \mathrm{~kg}$ de masa, rigidez de $14.437 \mathrm{~N} / \mathrm{m}$ y amortiguamiento de $132 \mathrm{Ns} / \mathrm{m}$.

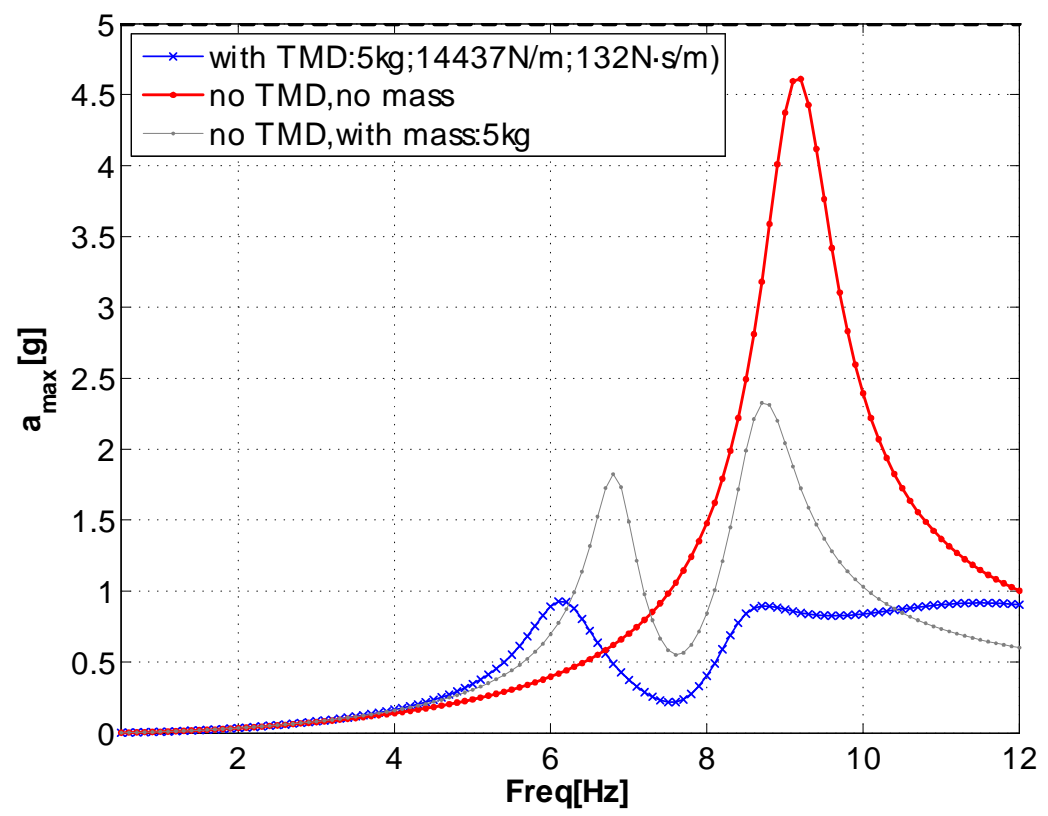

Figura 4-29: Respuesta en frecuencia de aceleraciones en punta de ala izquierda (amortiguamiento modal $5 \%$ )

La respuesta de aceleraciones verticales en el semiala izquierda es menor que para el caso de menor amortiguamiento como puede verse en las figuras 4-29, 4-30 y 4-31. 


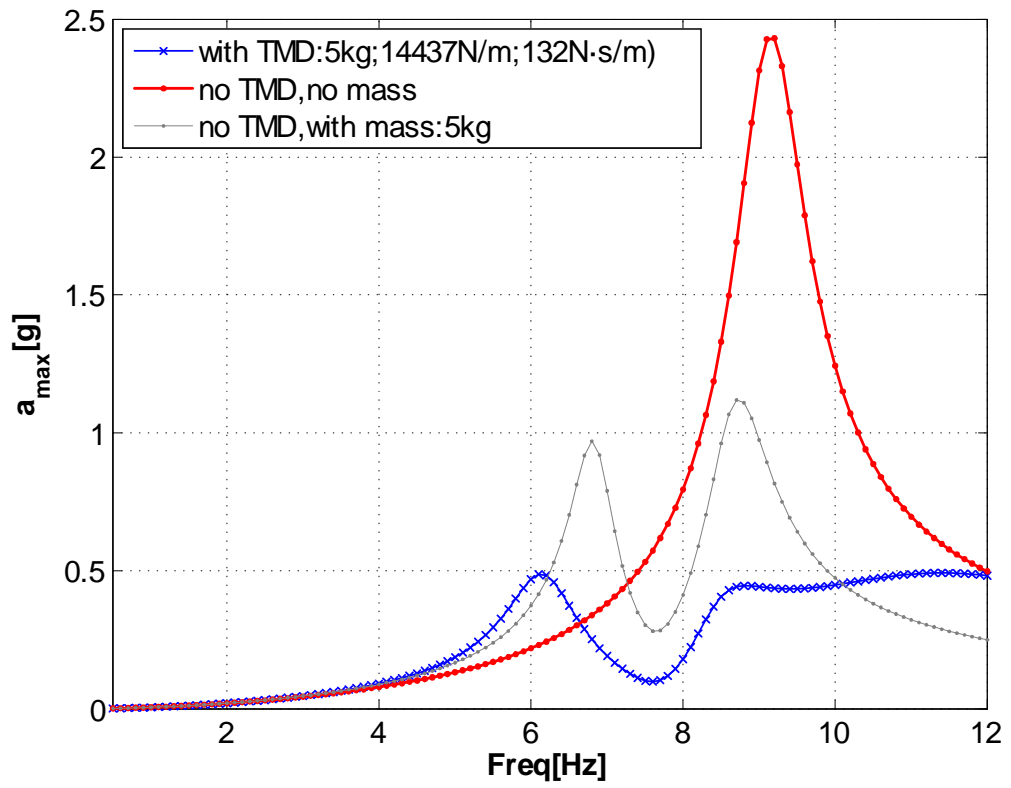

Figura 4-30: Respuesta en frecuencia de aceleraciones en inicio de estrechamiento de semiala izquierda (amortiguamiento modal 5\%)

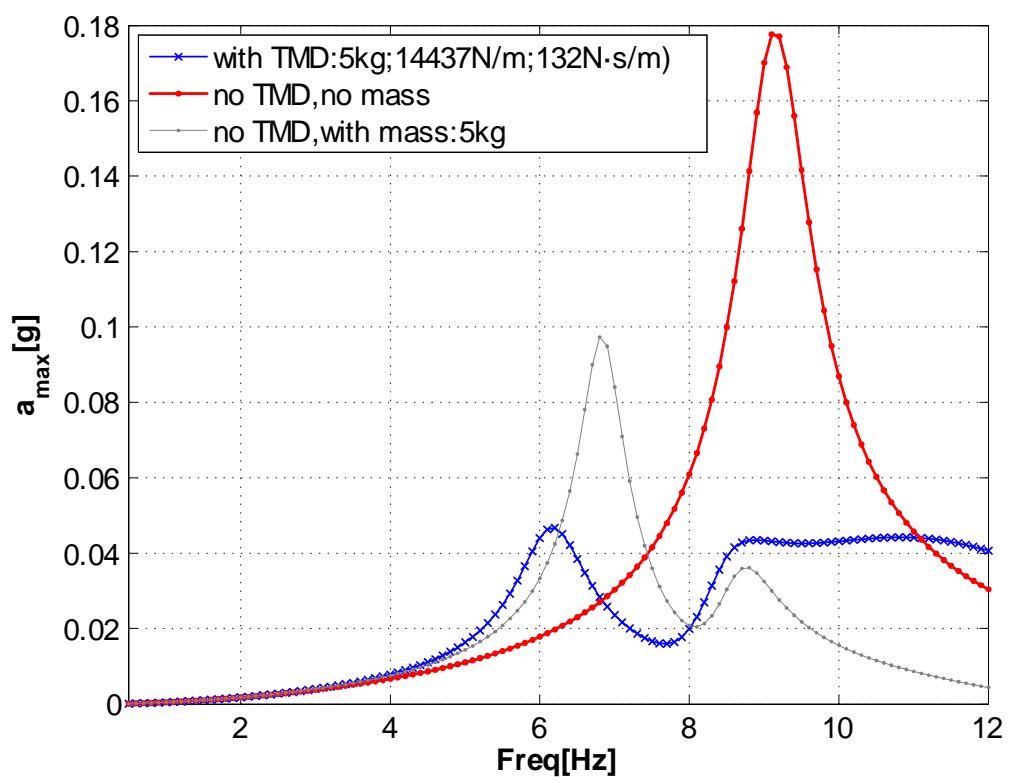

Figura 4-31: Respuesta en frecuencia de aceleraciones en encastre de semiala izquierda (amortiguamiento modal 5\%) 
El menor valor de los máximos de aceleraciones queda patente en las curvas de análisis paramétricos en masa (figura 4-32), de rigidez (figura 4-33) y de amortiguamiento (figura 4-34).

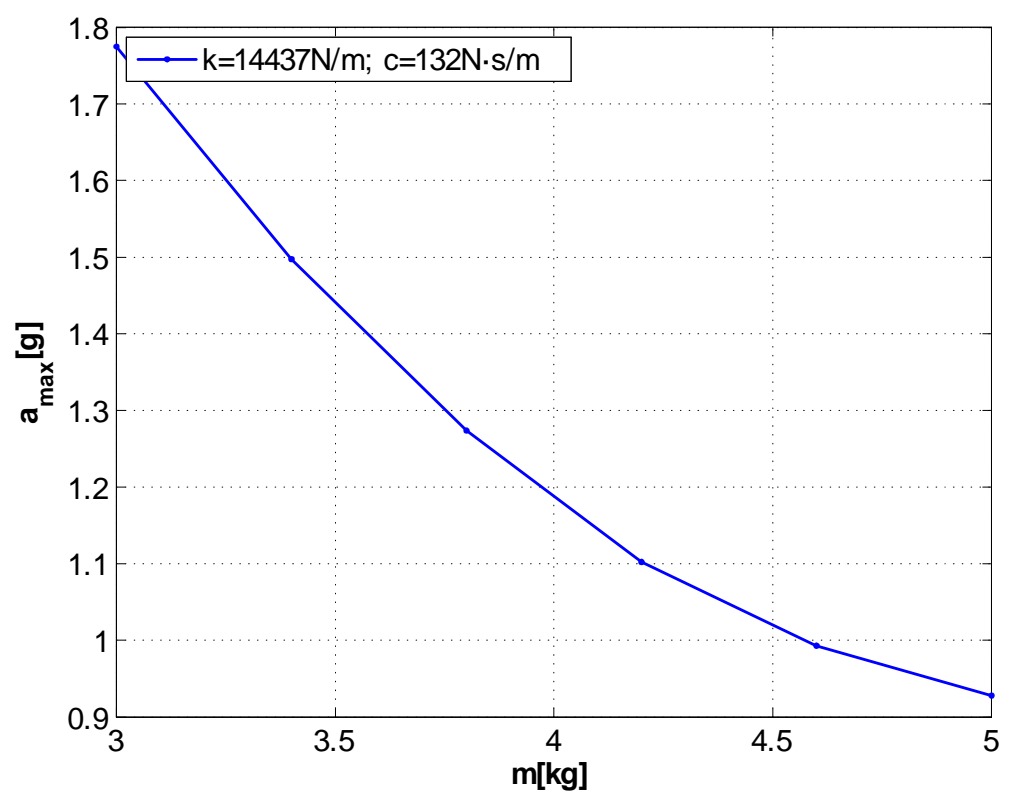

Figura 4-32: Análisis paramétrico en masa (amortiguamiento modal 5\%)

En la gráfica 4-33, puede comprobarse que además de ser menores los valores de aceleraciones, también el mínimo en función de la rigidez es mayor que para el caso de un amortiguamiento del 1\% como ya se ha comentado. En la figura 4-34 se verifica que el mínimo de aceleraciones en función del amortiguamiento es menor (ver figura 4-35) pero se corresponde con un valor superior de amortiguamiento (132 en vez de $81 \mathrm{Ns} / \mathrm{m}$ ). 


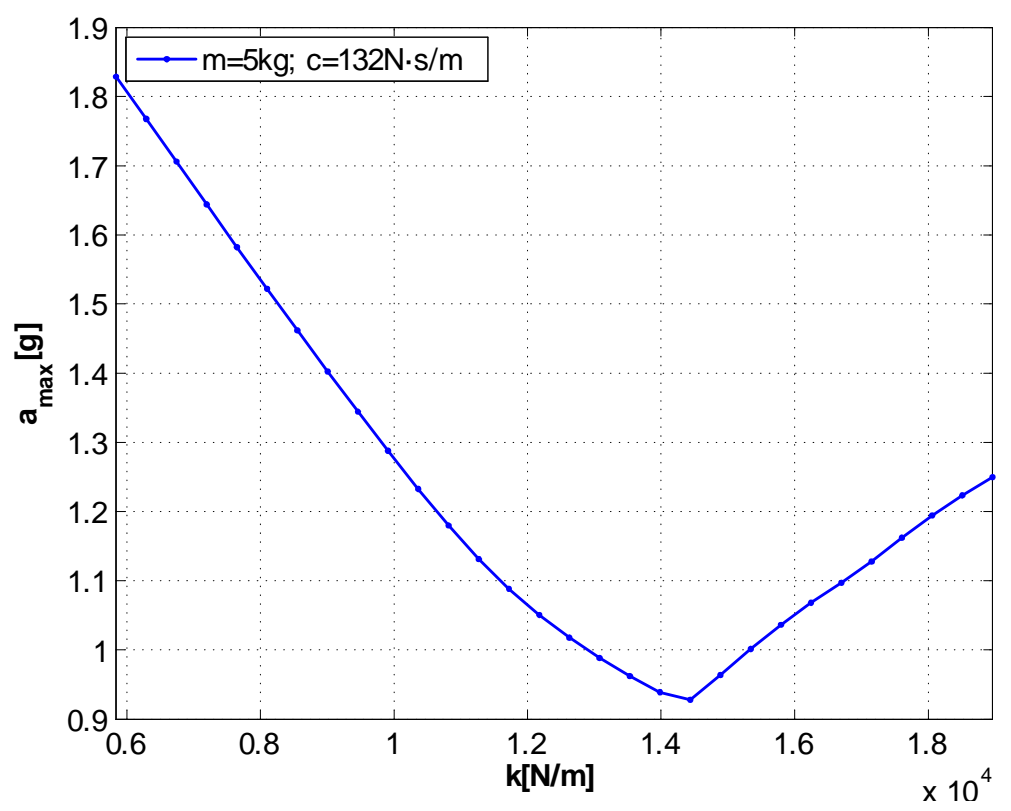

Figura 4-33: Análisis paramétrico en rigidez (amortiguamiento modal 5\%)

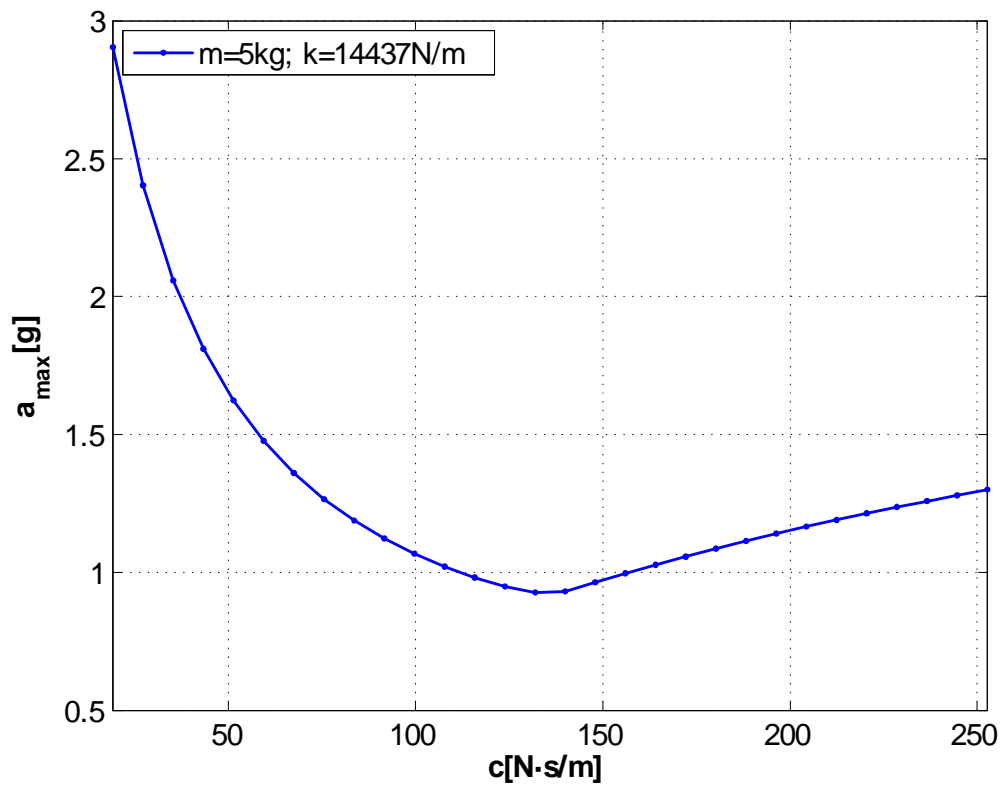

Figura 4-34: Análisis paramétrico en amortiguamiento (amortiguamiento modal 5\%) 


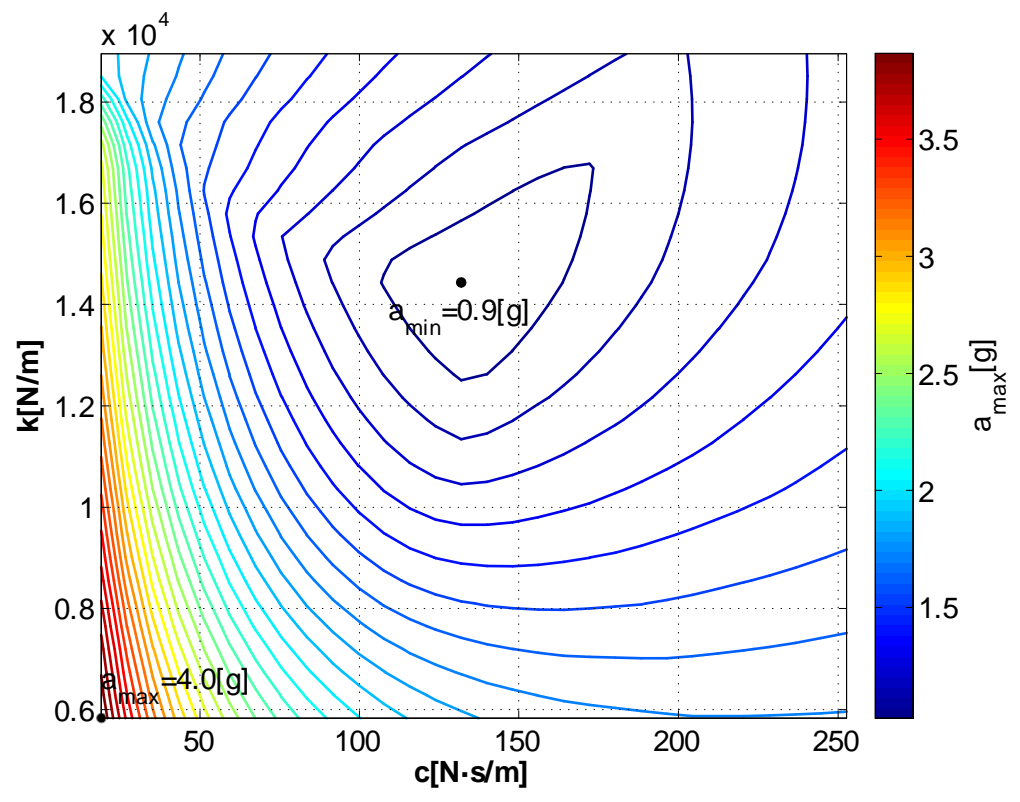

Figura 4-35: Gráfica de líneas isoparamétricas k-c (amortiguamiento modal 5\%)

Se muestra en la tabla 4.5 la comparación de los valores de los picos a $6.8 \mathrm{~Hz}$ y $8.7 \mathrm{~Hz}$ y el valle a 7.6 que permiten identificar la diferencia entre las cifras obtenidas en los análisis paramétricos empleando el método en estudio y los resultados obtenidos mediante el programa comercial de elementos finitos para el caso de adición de equipo sin incluir el TMD.

\begin{tabular}{|c|c|c|c|}
\hline Frecuencia $(\mathrm{Hz})$ & 6.8 & 7.6 & 8.7 \\
\hline Aceleración con método de aceleración $\left(\mathrm{m} / \mathrm{s}^{2}\right)$ & 17.9 & 5.4 & 22.8 \\
\hline Aceleración con programa comercial $\left(\mathrm{m} / \mathrm{s}^{2}\right)$ & 15.7 & 4.3 & 22.1 \\
\hline Diferencia & $14 \%$ & $26 \%$ & $3 \%$ \\
\hline
\end{tabular}

Tabla 4.5: Comparación de análisis sin TMD con amortiguamiento incrementado

Puede verse que las cifras de respuesta en aceleraciones obtenidas por el método de aceleración estudiado son ligeramente superiores a las producidas por el programa comercial. La mayor diferencia se registra en el mínimo a $7.6 \mathrm{~Hz}$ que resulta un $26 \%$.

En la tabla 4.6 se puede estudiar la comparación de los valores de los picos y valles que permiten identificar la diferencia entre las cifras análogas para el caso de adición de equipo 
incluyendo el TMD.

\begin{tabular}{|c|c|c|c|}
\hline Frecuencia $(\mathrm{Hz})$ & 6.1 & 7.6 & 8.4 \\
\hline Aceleración con método de aceleración $\left(\mathrm{m} / \mathrm{s}^{2}\right)$ & 9.1 & 2.1 & 8.8 \\
\hline Aceleración con programa comercial $\left(\mathrm{m} / \mathrm{s}^{2}\right)$ & 7.1 & 2.5 & 10.5 \\
\hline Diferencia & $29 \%$ & $14 \%$ & $16 \%$ \\
\hline
\end{tabular}

Tabla 4.6: Comparación de análisis con TMD con amortiguamiento incrementado

Puede verse en primer lugar que la adición del sistema de atenuación reduce la frecuencia de primer máximo de aceleraciones. En este caso las aceleraciones obtenidas por el método de aceleración de análisis muestran valores menores que las obtenidas por el programa comercial, siendo la diferencia máxima de un $29 \%$ que se produce en el máximo a $6.1 \mathrm{~Hz}$.

Por lo tanto, puede resumirse que las diferencias entre los resultados logrados mediante el método de aceleración de análisis y los obtenidos mediante el programa comercial realizado sobre el modelo de elementos finitos para un amortiguamiento modal del $5 \%$ no alcanzan un $30 \%$.

Se ha realizado una comparación análoga a la del apartado anterior respecto a análisis transitorios del modelo de elementos finitos de la estructura con el programa comercial para el caso de un amortiguamiento modal de un 5\%. El resultado se muestra en la figura 4-36.

A continuación se incluyen las figuras 4-37, 4-38 y 4-39 de la respuesta temporal (análisis implícito Newmark- $\beta$ ) en las frecuencias de los extremos dentro del rango de estudio con un amortiguamiento de un $5 \%$. 


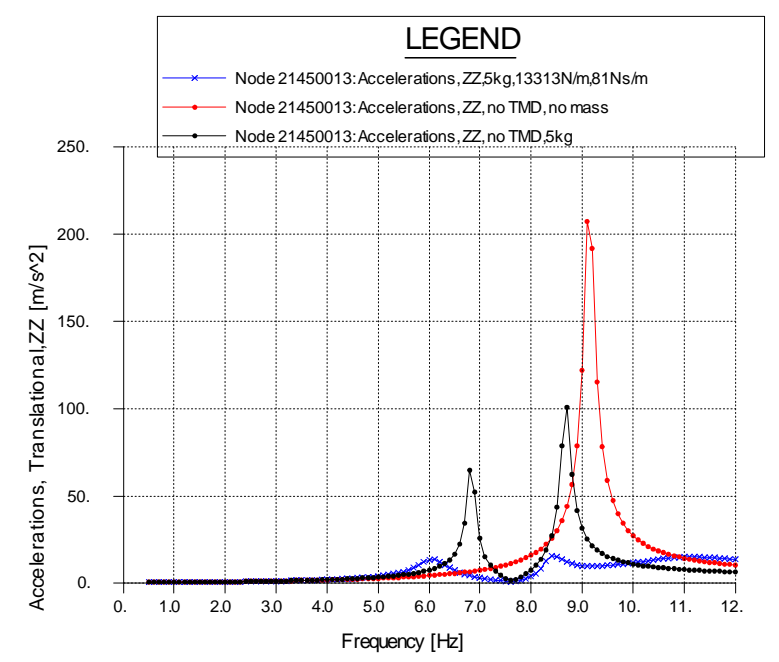

Figura 4-36: Respuesta en frecuencia de aceleración en punta de ala izquierda del modelo FEM (amortiguamiento modal 5\%)

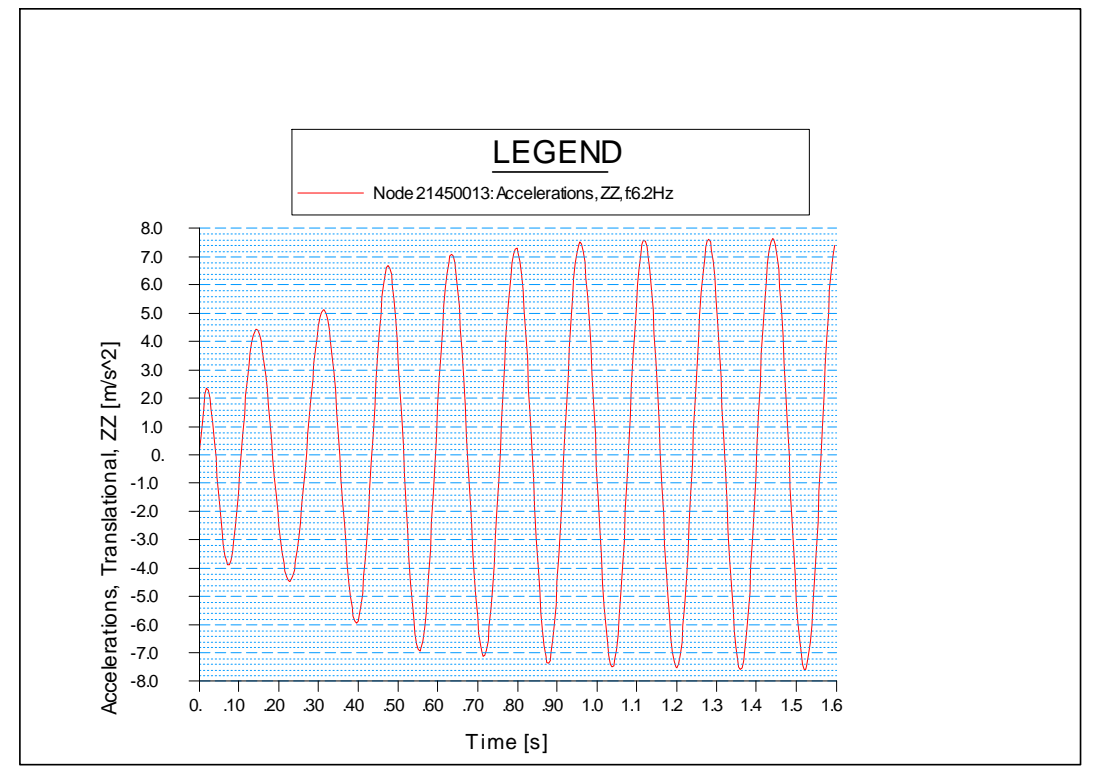

Figura 4-37: Respuesta temporal de aceleraciones en punta de ala izquierda a frecuencia $6.2 \mathrm{~Hz}$ (amortiguamiento modal 5\%) 


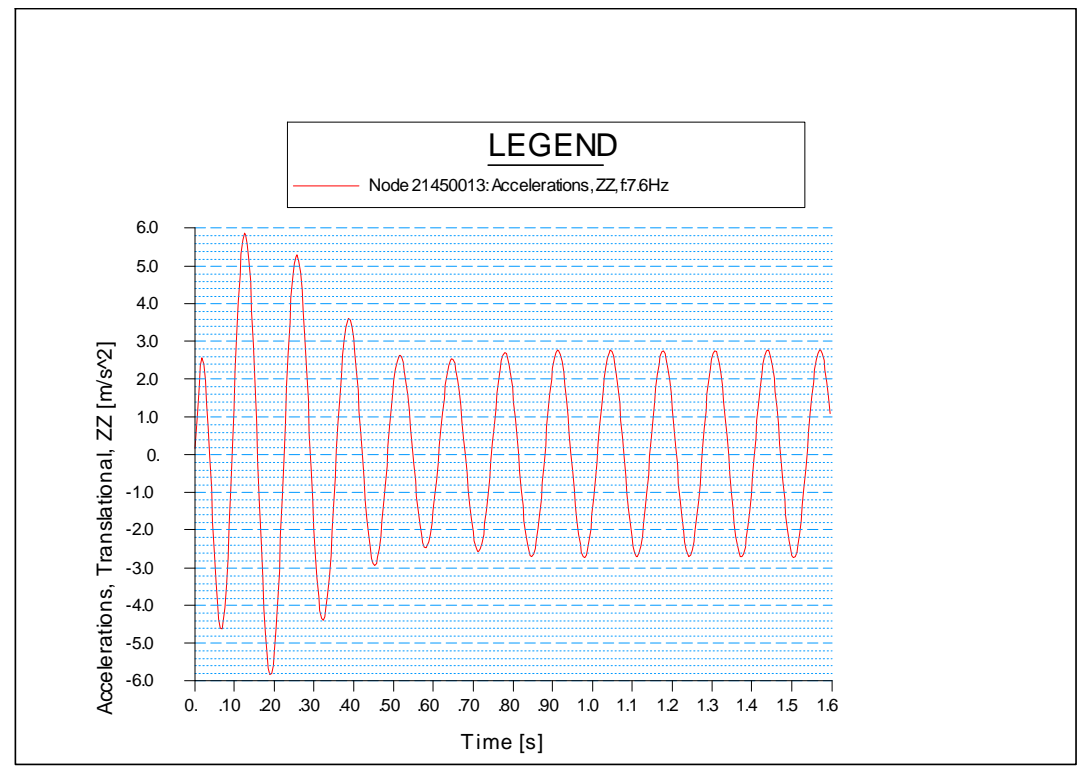

Figura 4-38: Respuesta temporal de aceleraciones en punta de ala izquierda a frecuencia $7.6 \mathrm{~Hz}$ (amortiguamiento modal 5\%)

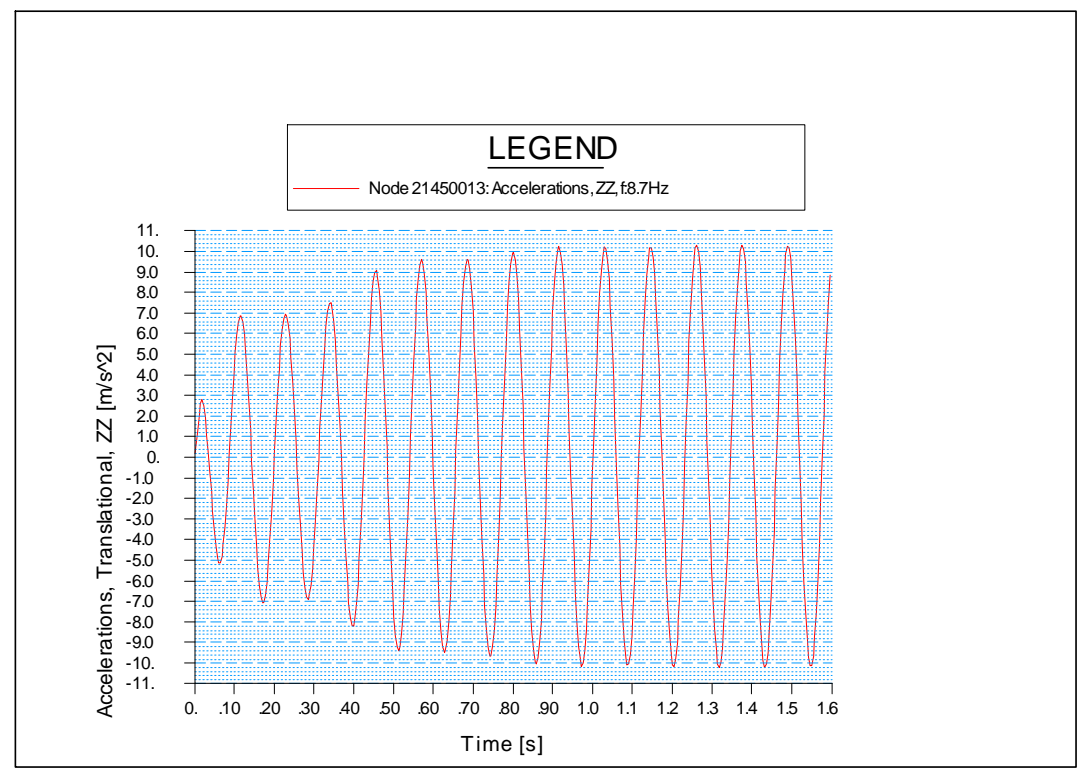

Figura 4-39: Respuesta temporal de aceleraciones en punta de ala izquierda a frecuencia $8.7 \mathrm{~Hz}$ (amortiguamiento modal 5\%) 
Se observa que las respuestas son más estables que en el caso de $1 \%$ de amortiguamiento. La comparación de los valores en los extremos es la siguiente:

\begin{tabular}{|c|c|c|c|}
\hline Frecuencia $(\mathrm{Hz})$ & $6.1(6.2)$ & 7.6 & 8.7 \\
\hline Aceleración con método de aceleración $\left(\mathrm{m} / \mathrm{s}^{2}\right)$ & 9.1 & 2.1 & 8.8 \\
\hline Aceleración con programa comercial $\left(\mathrm{m} / \mathrm{s}^{2}\right)$ & 7.7 & 2.7 & 10.2 \\
\hline Diferencia & $18 \%$ & $20 \%$ & $14 \%$ \\
\hline
\end{tabular}

Tabla 4.7: Comparación de máximos de aceleraciones con análisis transitorio con amortiguamiento incrementado

Hay que resaltar que el máximo obtenido por el método de aceleración de análisis se encuentra a una frecuencia de $6.1 \mathrm{~Hz}$ y por lo tanto ligeramente distinta al valor de la frecuencia del máximo calculado con el programa comercial que es $6.2 \mathrm{~Hz}$ lo que puede producir que los resultados difieran por esta causa. Los valores de los máximos obtenidos con el análisis realizado sobre el modelo de elementos finitos con el programa comercial difieren como máximo en un $20 \%$.

En resumen, se puede decir que los resultados con un amortiguamiento del $5 \%$ presentan diferencias mayores que los calculados con un $1 \%$ respecto a los resultados proporcionados por el programa comercial de elementos finitos.

Dada la posible influencia del amortiguamiento modal, se ha estudiado una solución con amortiguamiento nulo. Para ello, se han realizado unos análisis paramétricos de respuesta en frecuencia con las siguientes variaciones:

- Masa del equipo incluyendo su anclaje: $3 \mathrm{~kg}$ a $5 \mathrm{~kg}$ en 6 escalones iguales

- Rigidez del anclaje: 9720 N/m a 18965 N/m en 30 escalones iguales

- Amortiguamiento de la unión: 19.1 Ns/m a 252.7 Ns/m en 30 escalones iguales

Como se realiza un análisis para cada variación de cada uno de los parámetros se ejecutan un total de 5400 análisis con 116 frecuencias entre 0.5 y $20 \mathrm{~Hz}$. Para identificar las diferencias se ha considerado un amortiguamiento modal nulo en la estructura no modificada. 


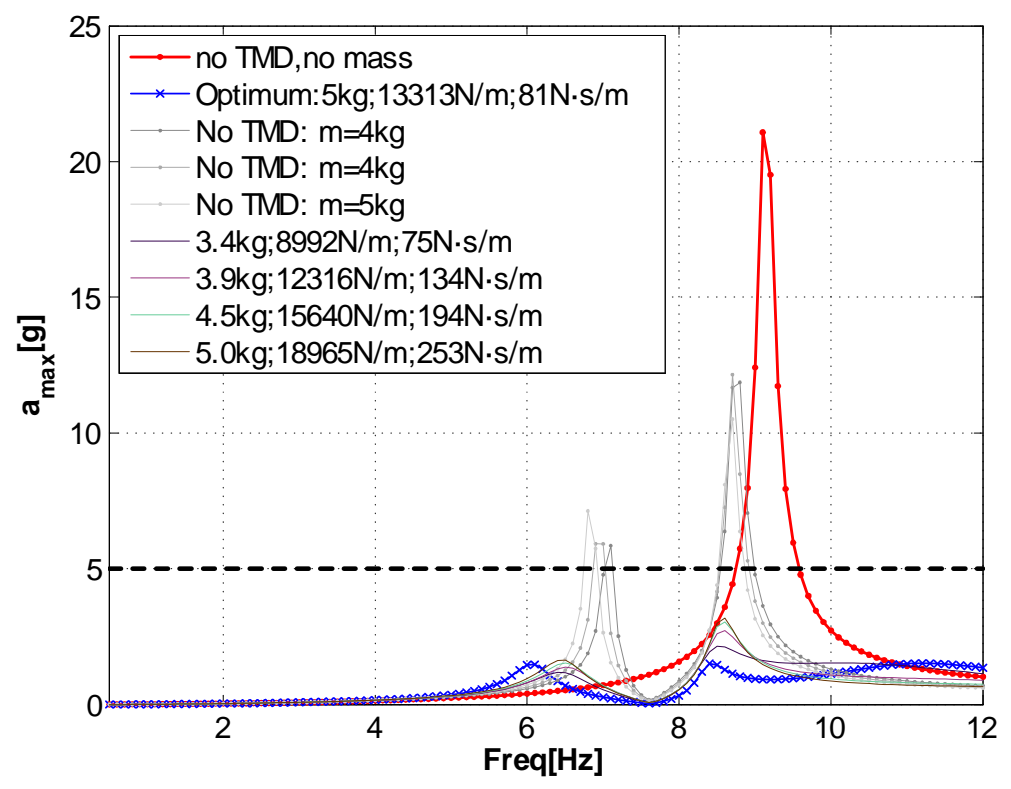

Figura 4-40: Análisis paramétrico. Resumen de respuestas en aceleraciones en punta de ala izquierda (amortiguamiento modal nulo)

$\mathrm{Al}$ contrario que en el caso anterior en el que se aumentaba el amortiguamiento, la aceleración vertical en punta de ala izquierda se dispara en algunos de los casos sin TMD (ver figura 4-40). También se observa que el conjunto de valores óptimos de propiedades del sistema de amortiguación es distinto, alcanzándose para $5 \mathrm{~kg}$ de masa, rigidez de $12.173 \mathrm{~N} / \mathrm{m}$ y amortiguamiento de $59 \mathrm{Ns} / \mathrm{m}$. En la figura 4-41 puede verse el detalle de la aceleración en punta de ala izquierda de los casos: sin equipo añadido, con equipo añadido pero sin TMD y con equipo añadido con TMD. Se aprecia el elevado valor de la aceleración sin equipo al no ofrecer la estructura amortiguamiento.

$\mathrm{Al}$ acercarnos al encastre del ala la respuesta se va reduciendo (ver figuras 4-42 y 4-43) pero sigue siendo mayor que para el caso con amortiguamiento.

El análisis paramétrico en masa se muestra en la figura 4-44. La variación de la aceleración en punta de ala izquierda ofrece el mínimo con la rigidez a $12173 \mathrm{~N} / \mathrm{m}$ (ver figura 4-45) inferior al correspondiente del caso de $1 \%$ de amortiguamiento. 


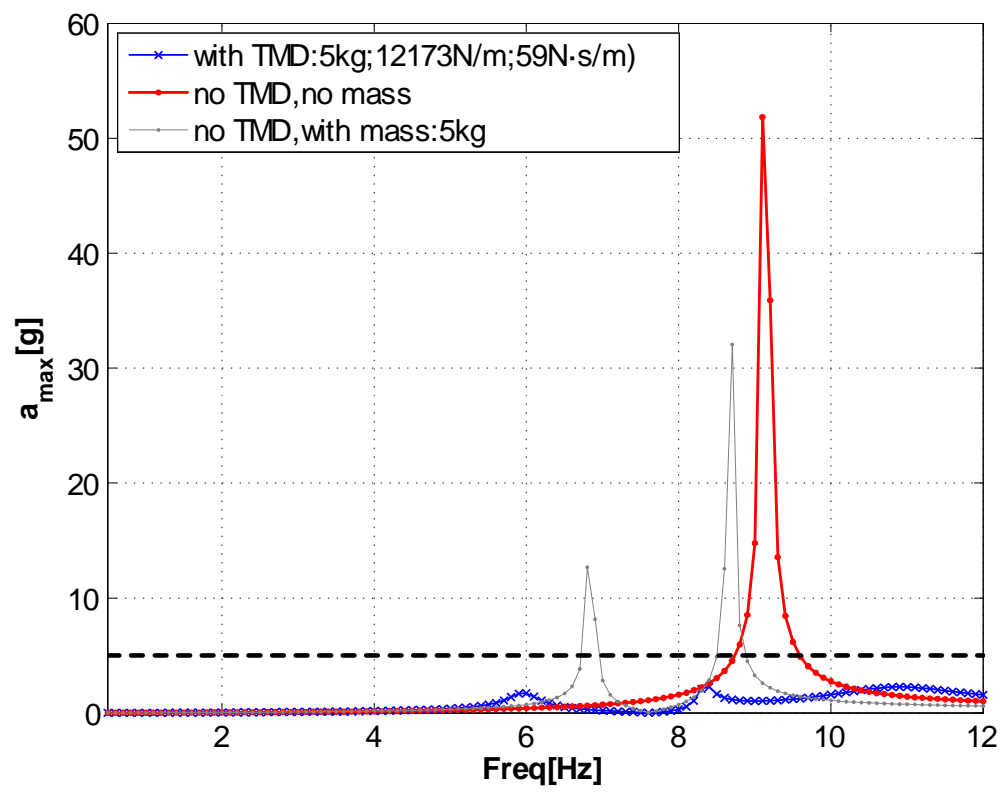

Figura 4-41: Respuesta en frecuencia de aceleraciones en punta de semiala izquierda (amortiguamiento modal nulo)

De manera análoga, la aceleración presenta el mínimo a un amortiguamiento de $59 \mathrm{Ns} / \mathrm{m}$ (4-46) claramente menor que para el caso de $1 \%$ de amortiguamiento que sucede a $81 \mathrm{Ns} / \mathrm{m}$.

El mínimo de aceleraciones de la combinación de parámetros de rigidez y amortiguamiento se muestra en la figura 4-47 y corresponde a una aceleración de $2.3 \mathrm{~g}$ superior al caso de $1 \%$ de amortiguamiento. 


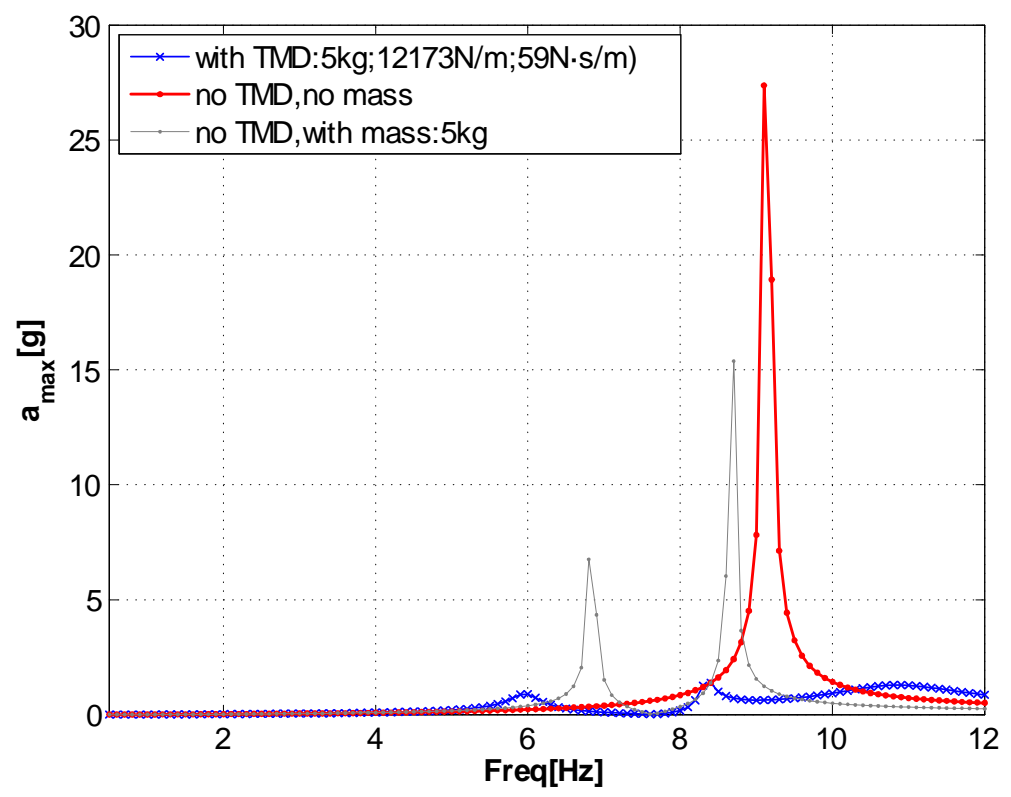

Figura 4-42: Respuesta en frecuencia de aceleraciones en inicio de estrechamiento de semiala izquierda (amortiguamiento modal nulo)

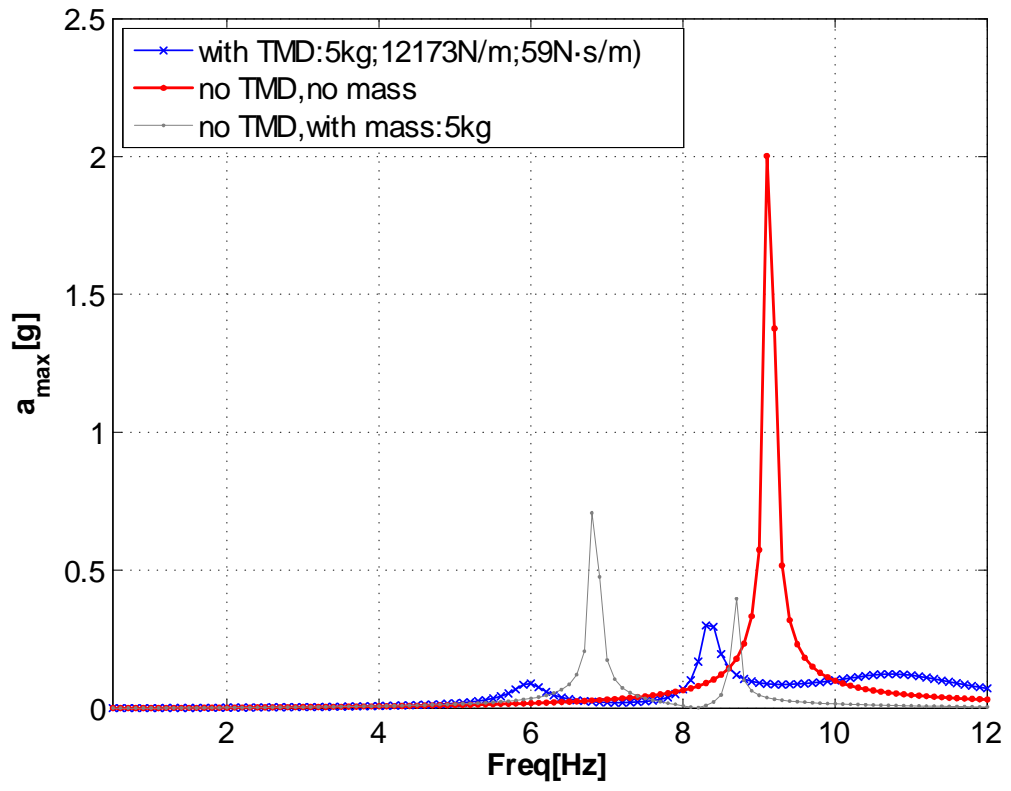

Figura 4-43: Respuesta en frecuencia de aceleraciones en encastre de semiala izquierda (amortiguamiento modal nulo) 


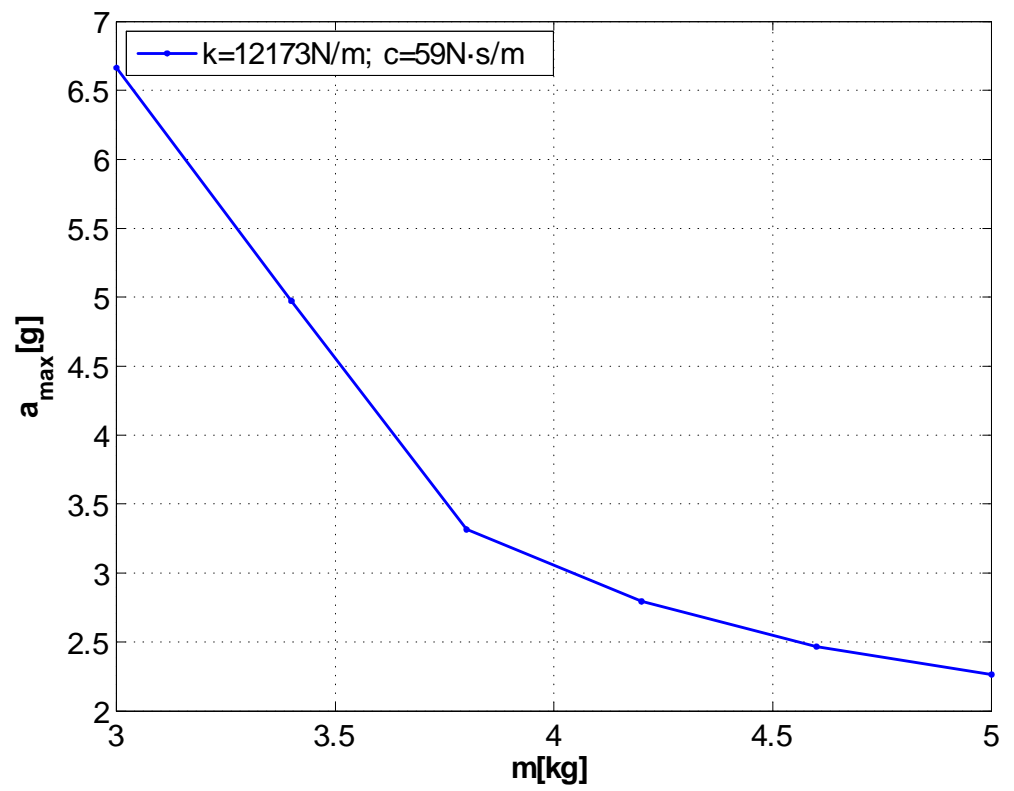

Figura 4-44: Análisis paramétrico en masa (amortiguamiento modal nulo)

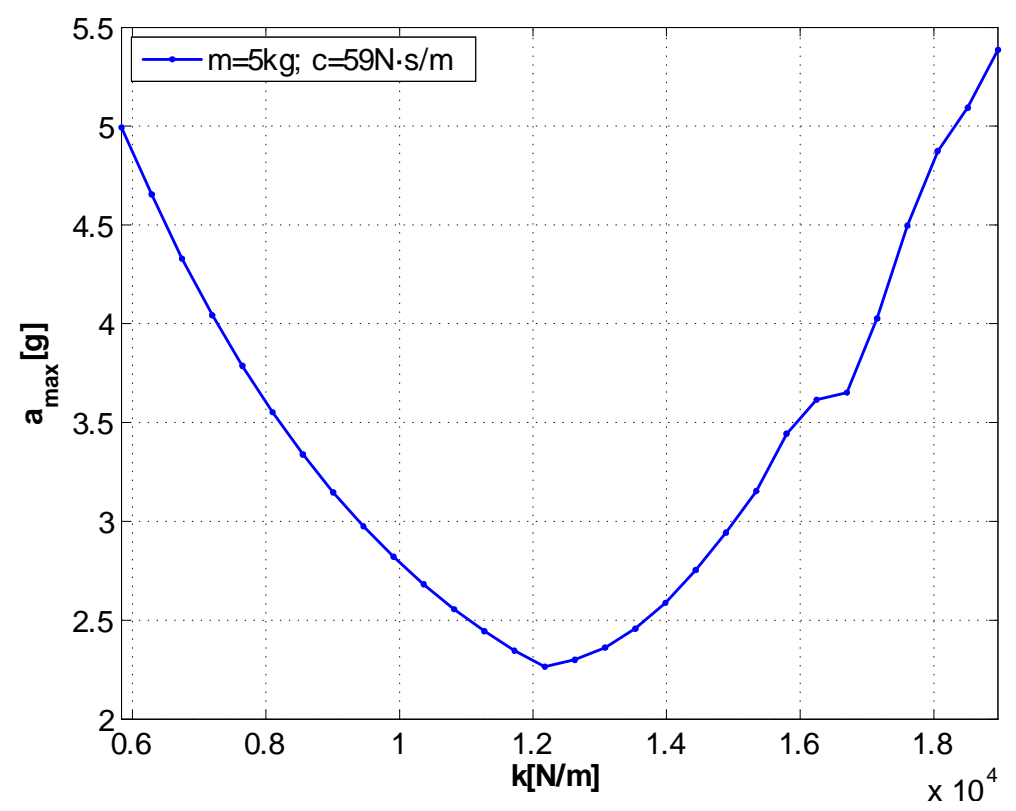

Figura 4-45: Análisis paramétrico en rigidez (amortiguamiento modal nulo) 


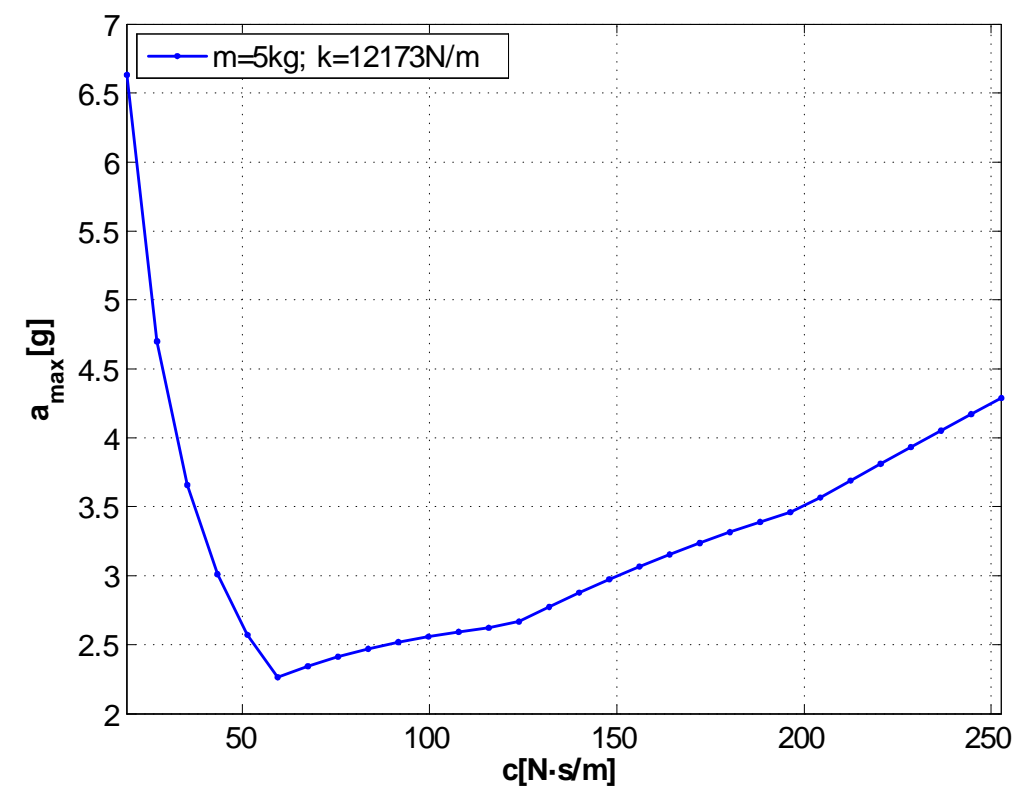

Figura 4-46: Análisis paramétrico en amortiguamiento (amortiguamiento modal nulo)

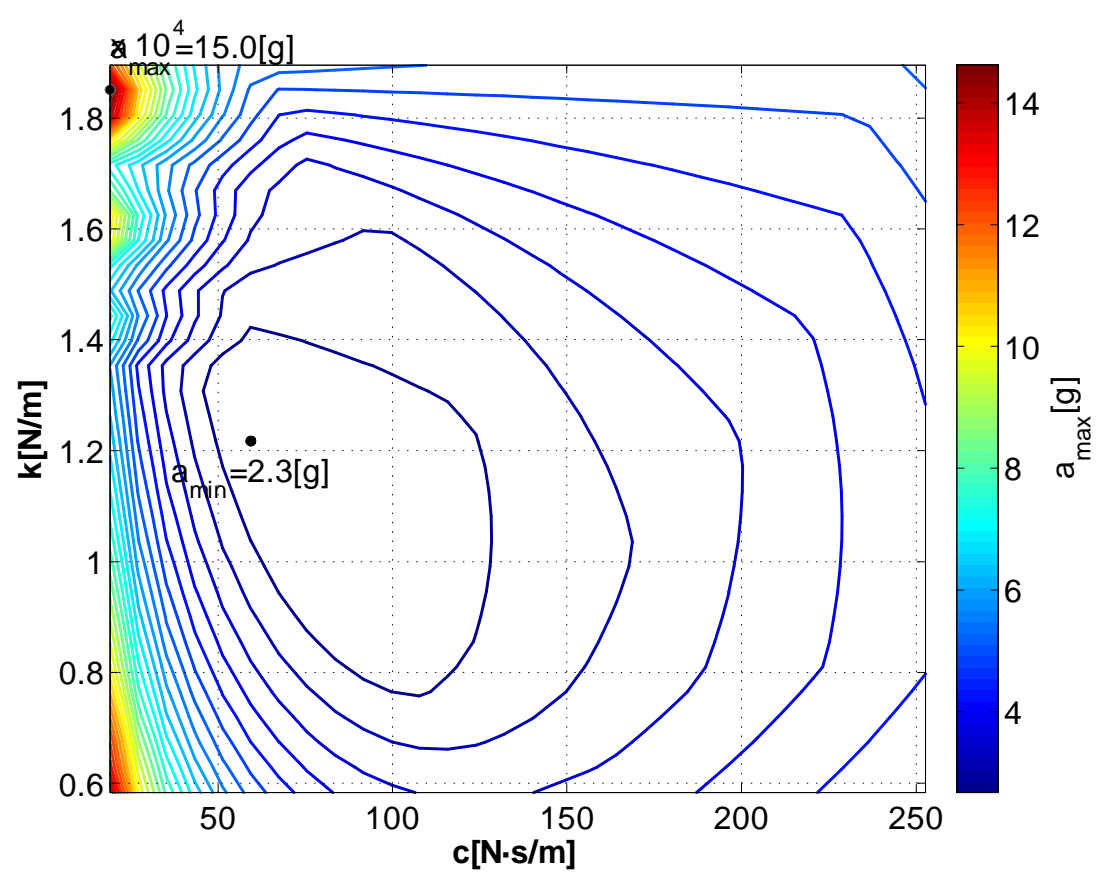

Figura 4-47: Gráfica de líneas isoparamétricas k-c (amortiguamiento modal nulo) 
Se muestra en la tabla 4.8 la comparación de los valores de los picos a $6.8 \mathrm{~Hz}$ y $8.7 \mathrm{~Hz}$ y el valle a 7.6 que permiten identificar la diferencia entre las cifras obtenidas en los análisis paramétricos empleando el método en estudio y los resultados obtenidos mediante el programa comercial de elementos finitos para el caso de adición de equipo sin incluir el TMD.

\begin{tabular}{|c|c|c|c|}
\hline Frecuencia $(\mathrm{Hz})$ & 6.8 & 7.6 & 8.7 \\
\hline Aceleración con método de aceleración $\left(\mathrm{m} / \mathrm{s}^{2}\right)$ & 124.2 & 0.2 & 314.3 \\
\hline Aceleración con programa comercial $\left(\mathrm{m} / \mathrm{s}^{2}\right)$ & 128.6 & 0.2 & 308.5 \\
\hline Diferencia & $3 \%$ & $0 \%$ & $2 \%$ \\
\hline
\end{tabular}

Tabla 4.8: Comparación de análisis sin TMD con amortiguamiento nulo

Puede verse que las cifras de respuesta en aceleraciones obtenidas por el método de aceleración estudiado son similares a las producidas por el programa comercial, siendo la mayor diferencia de un $3 \%$.

En la tabla 4.9 se puede estudiar la comparación de los valores de los picos y valles que permiten identificar la diferencia entre las cifras análogas para el caso de adición de equipo incluyendo el TMD.

\begin{tabular}{|c|c|c|c|}
\hline Frecuencia $(\mathrm{Hz})$ & 6.1 & 7.6 & 8.4 \\
\hline Aceleración con método de aceleración $\left(\mathrm{m} / \mathrm{s}^{2}\right)$ & 16.7 & 0.0 & 22.2 \\
\hline Aceleración con programa comercial $\left(\mathrm{m} / \mathrm{s}^{2}\right)$ & 16.8 & 0.0 & 22.0 \\
\hline Diferencia & $0 \%$ & $1 \%$ & $1 \%$ \\
\hline
\end{tabular}

Tabla 4.9: Comparación de análisis con TMD con amortiguamiento nulo

Puede verse en primer lugar que la adición del sistema de atenuación reduce la frecuencia de primer máximo de aceleraciones. En este caso las aceleraciones obtenidas por el método de aceleración de análisis muestran diferencias máximas respecto a las obtenidas por el programa comercial de un $1 \%$.

Por lo tanto, puede resumirse que las diferencias entre los resultados logrados mediante el método de aceleración de análisis y los obtenidos mediante el programa comercial realizado sobre el modelo de elementos finitos para un amortiguamiento modal nulo son despreciables. 
Para completar la comparación se adjuntan las figuras 4-48, 4-49 y 4-50 que recogen las respuestas de análisis transitorios realizados por medio de integraciones de acuerdo con el método implícito Newmark- $\beta$ sin amortiguamiento adicional al del TMD (c=0\%) para las frecuencias de los extremos del intervalo.

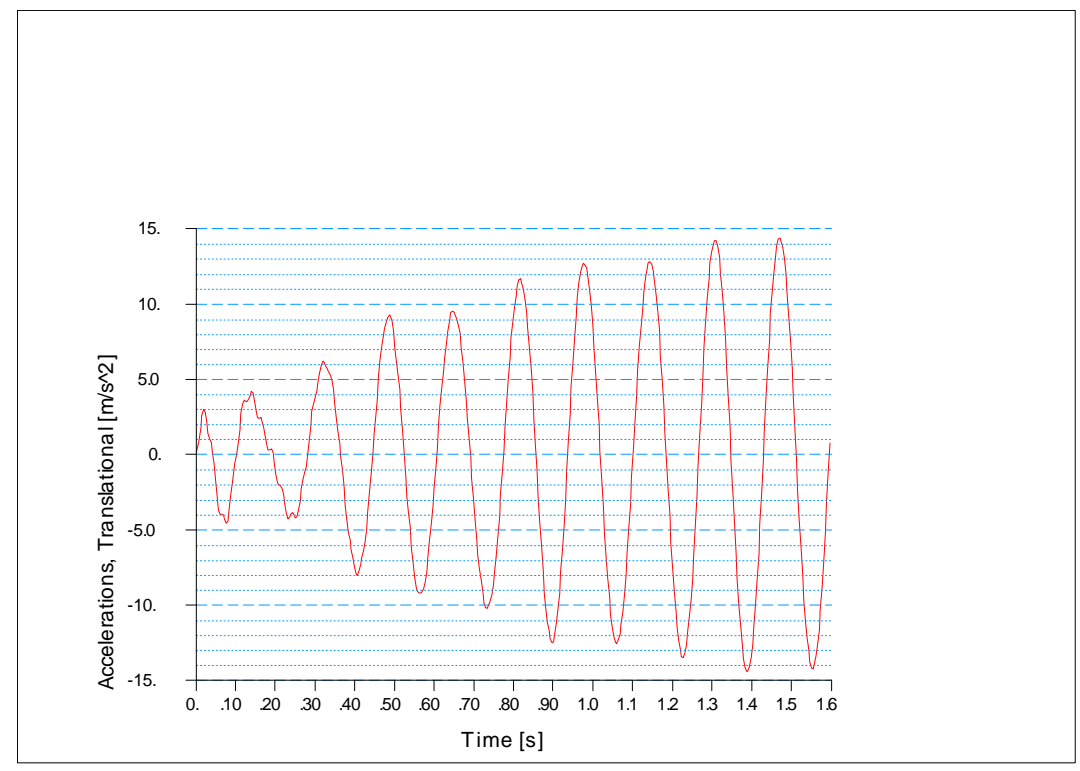

Figura 4-48: Respuesta temporal de aceleraciones en punta de ala izquierda a frecuencia $6.1 \mathrm{~Hz}$ para amortiguamiento modal $0 \%$ 


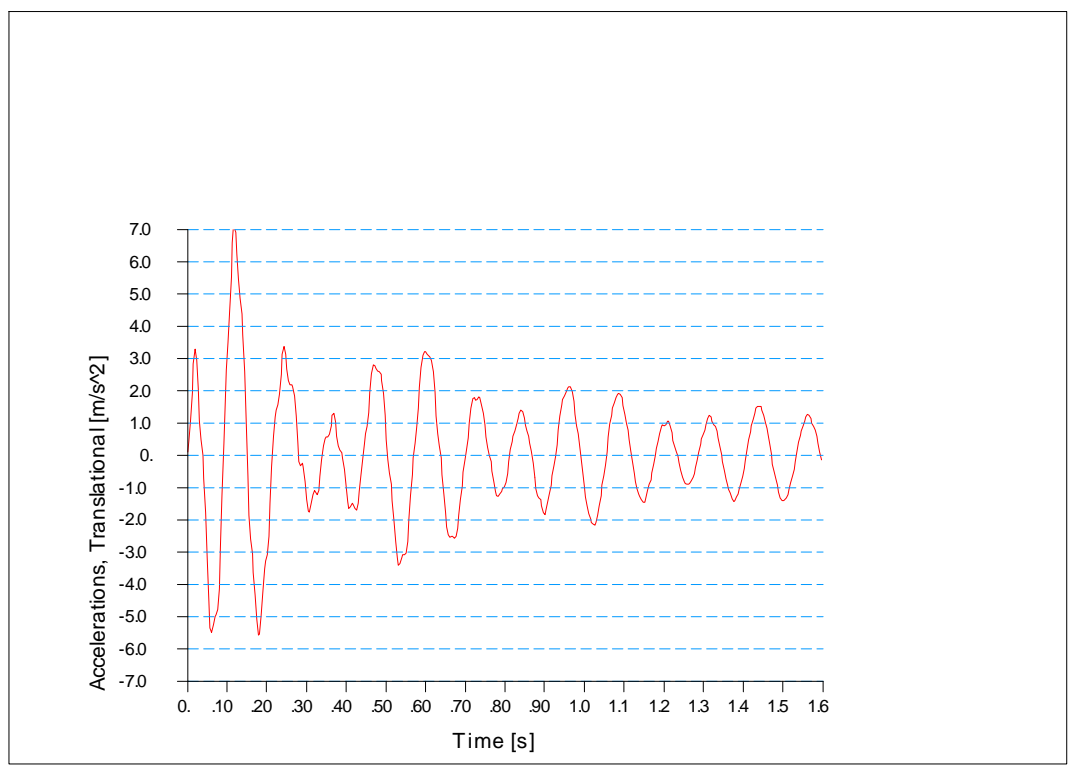

Figura 4-49: Respuesta temporal de aceleraciones en punta de ala izquierda a frecuencia $7.6 \mathrm{~Hz}$ para amortiguamiento modal $0 \%$

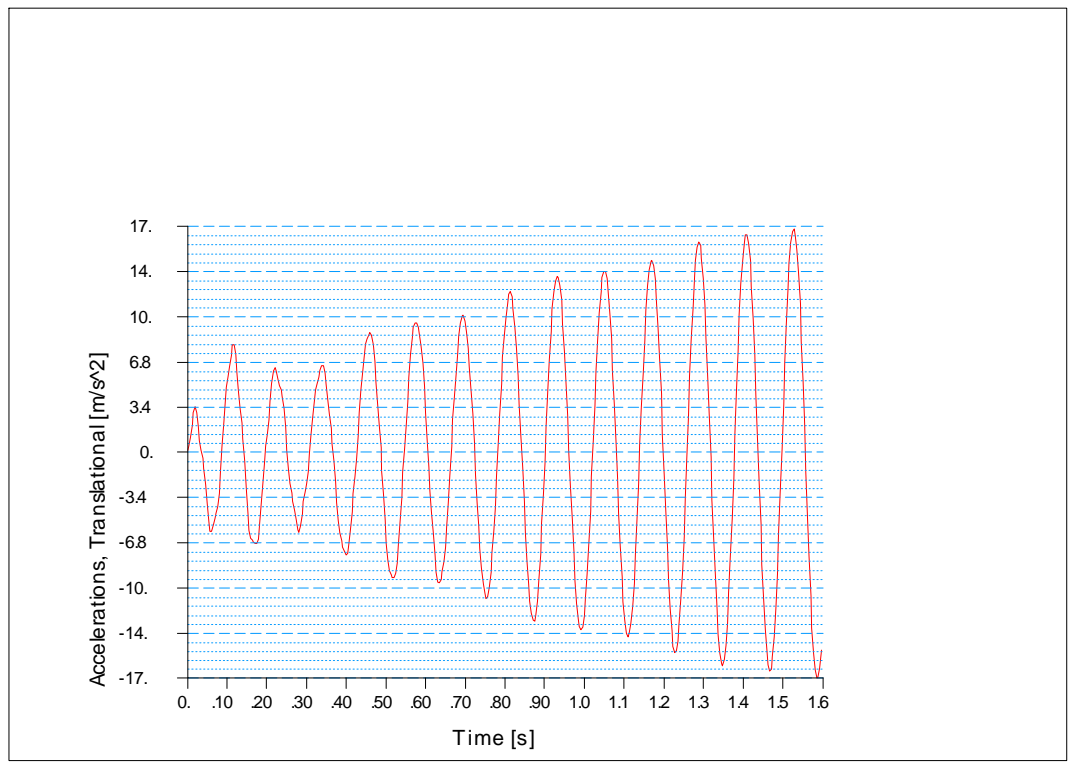

Figura 4-50: Respuesta temporal de aceleraciones en punta de ala izquierda a frecuencia $8.4 \mathrm{~Hz}$ para amortiguamiento modal $0 \%$ 
Puede observarse que, aunque la respuesta no está totalmente estabilizada a pesar de haber empleado varios ciclos, se puede considerar un valor de $14.5 \mathrm{~m} / \mathrm{s}^{2}$ como aceleración vertical en la punta de ala izquierda para la frecuencia de $6.1 \mathrm{~Hz}$. Hay que recordar que el máximo obtenido por el método de aceleración de análisis se encuentra a una frecuencia de $6.0 \mathrm{~Hz}$ y por lo tanto ligeramente distinta que puede producir que los resultados difieran por esta causa. Los valores a $7.6 \mathrm{~Hz}$ y $8.4 \mathrm{~Hz}$ son los de la tabla.

\begin{tabular}{|c|c|c|c|}
\hline Frecuencia $(\mathrm{Hz})$ & $6.0(6.1)$ & 7.6 & 8.4 \\
\hline Aceleración con método de aceleración $\left(\mathrm{m} / \mathrm{s}^{2}\right)$ & 16.7 & 0.05 & 22.21 \\
\hline Aceleración con programa comercial $\left(\mathrm{m} / \mathrm{s}^{2}\right)$ & 14.5 & 1.4 & 17 \\
\hline Diferencia & $15 \%$ & $97 \%$ & $31 \%$ \\
\hline
\end{tabular}

Tabla 4.10: Comparación de máximos de aceleraciones con análisis transitorio con amortiguamiento nulo

Puede verse que el valor más estabilizado ofrece un resultado más similar al del programa comercial. El valor a $7.6 \mathrm{~Hz}$ presenta grandes variaciones.

Teniendo en cuenta los estudios anteriores y la posible influencia del amortiguamiento de la estructura se puede realizar una parametrización también en el parámetro que lo define. En la figura 4-51 se muestra la variación de la aceleración en la punta de ala con el amortiguamiento modal.

Puede comprobarse que la aceleración disminuye al aumentar el parámetro de amortiguamiento modal. 


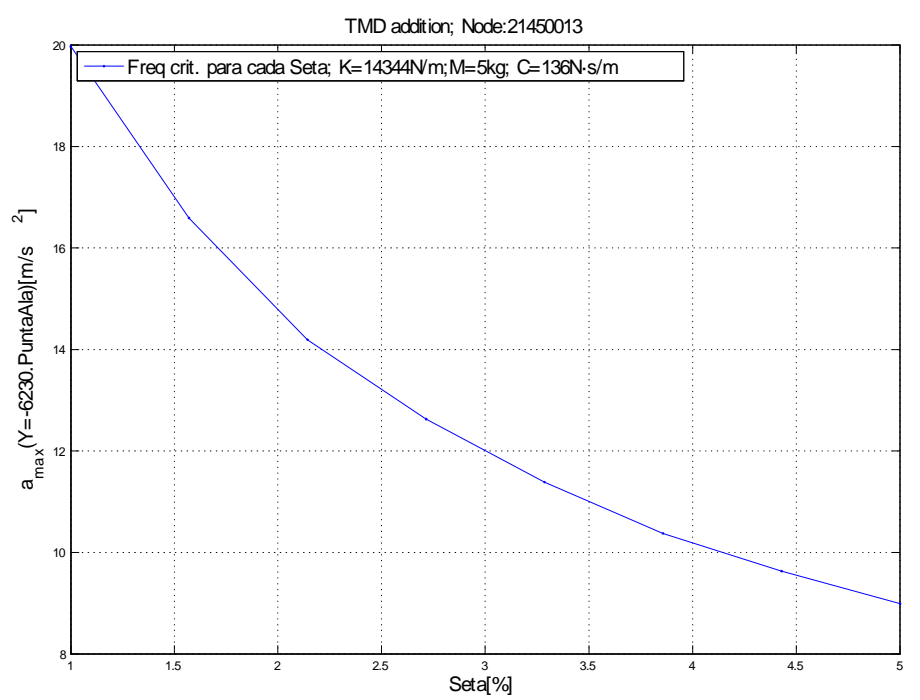

Figura 4-51: Análisis paramétrico en amortiguamiento modal

\subsection{Influencia del número de modos considerados}

Uno de los posibles factores de influencia en la precisión de las soluciones proporcionadas por el método de reducción en estudio puede ser el número de modos retenidos en la truncatura modal. Para entender el impacto en la elección de menos modos se han realizado diversos análisis variando el número de modos retenidos (21, 60, 100 y 151 modos) y se ha comparado los resultados obtenidos con los del programa de elementos finitos. Los resultados de referencia del apartado "Resultados del análisis paramétrico" se han calculado considerando 151 modos.

En primer lugar se muestran los resultados correspondientes a 21 modos con frecuencias entre 0 y $100 \mathrm{~Hz}$. En la figura 4-52 se ofrece una muestra de los resultados de los 5400 análisis realizados. Basándose en ellos se puede calcular la curva óptima de atenuación de respuesta en frecuencia del equipo que para este caso difiere respecto a los resultados calculados con más modos ya que se logran con una masa de $5 \mathrm{~kg}$, una rigidez de $13531 \mathrm{~N} / \mathrm{m}$ y un amortiguamiento de $84 \mathrm{Ns} / \mathrm{m}$.

En cambio, los resultados de aceleraciones no muestran grandes diferencias a pesar de la distinta sintonización del TMD (ver figuras 4-53, 4-54, 4-55) 


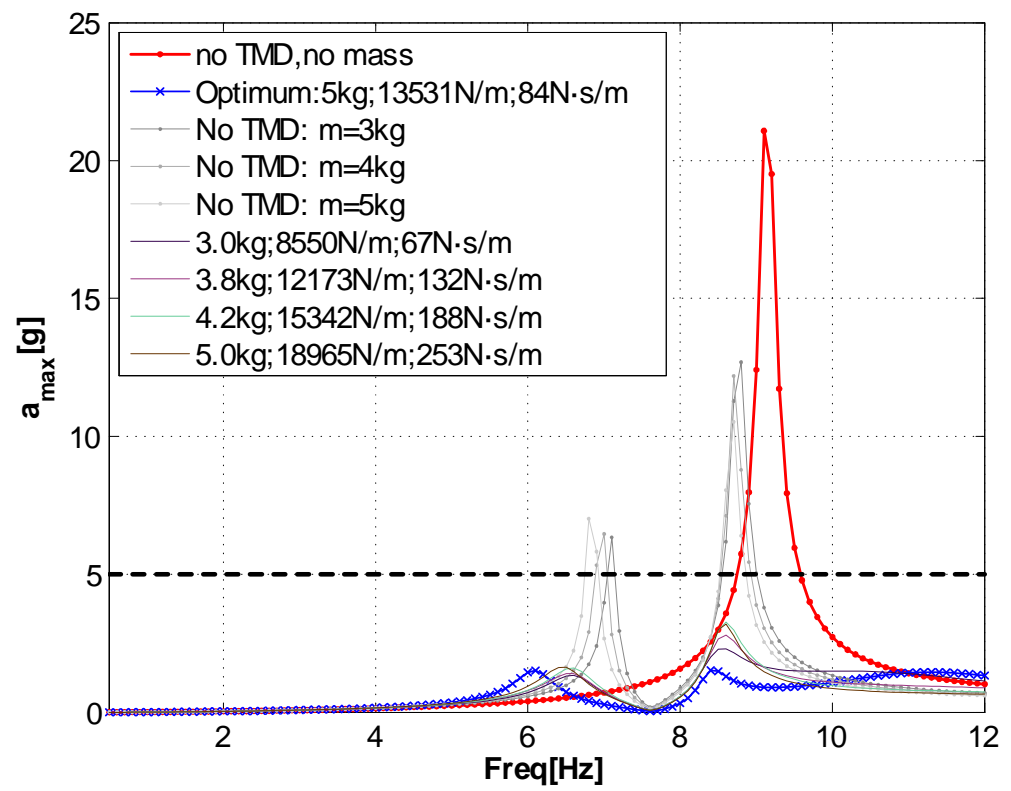

Figura 4-52: Análisis paramétrico. Resumen de respuesta en frecuencia de aceleraciones en punta de ala izquierda (truncatura 21 modos)

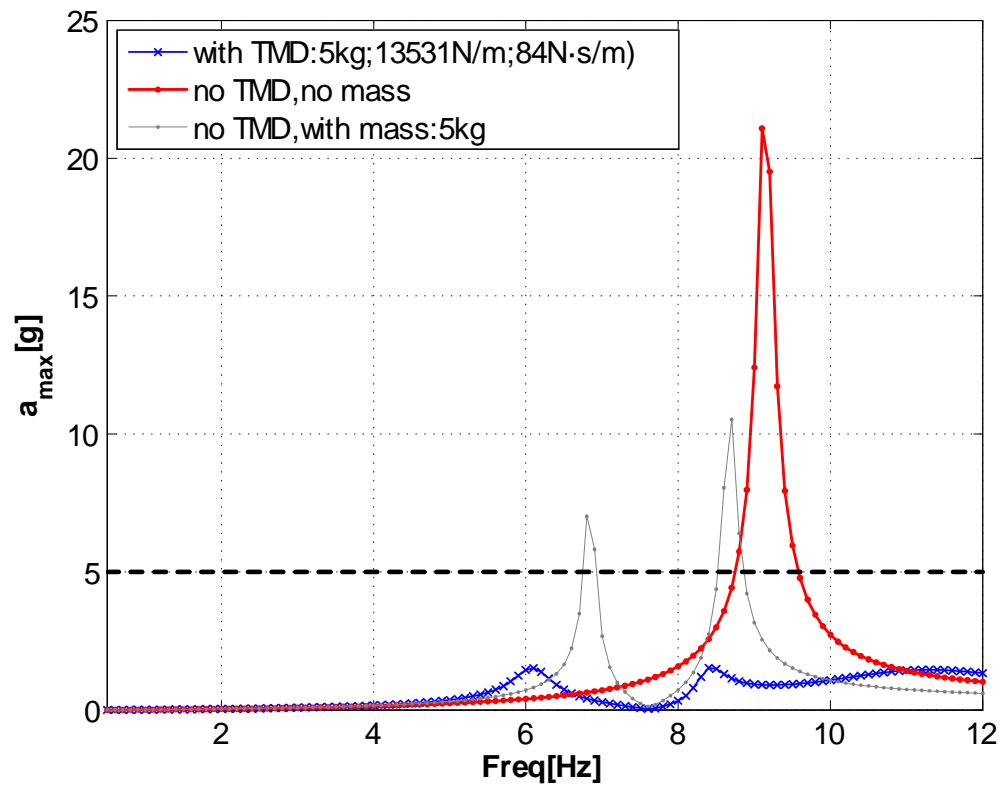

Figura 4-53: Respuesta en frecuencia de aceleraciones en punta de ala izquierda (truncatura 21 modos) 


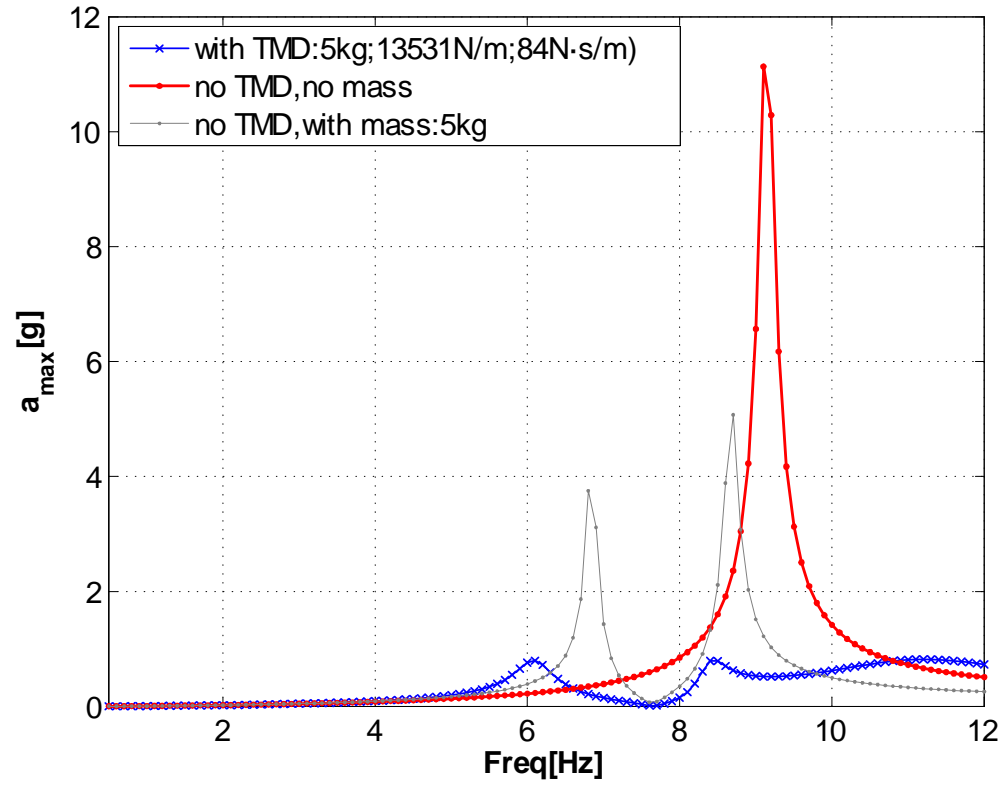

Figura 4-54: Respuesta en frecuencia de aceleraciones en inicio de estrechamiento en semiala izquierda (truncatura 21 modos)

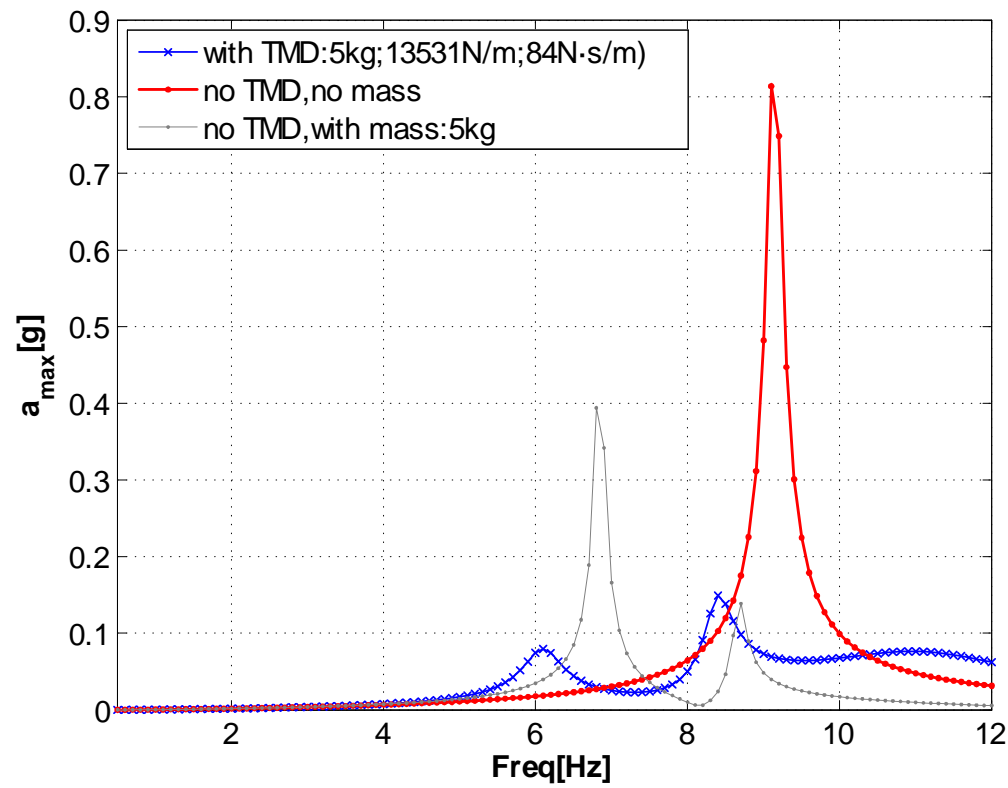

Figura 4-55: Respuesta en frecuencia de aceleraciones en encastre de semiala izquierda (truncatura 21 modos) 
En la figura 4-56 se muestra el análisis paramétrico con variación en la masa del conjunto equipo más dispositivo de atenuación. Puede verse en la figura 4-57 que el mínimo de aceleraciones respecto a la variación de rigidez es mucho más suave que en el cálculo realizado con la mayor truncatura modal.

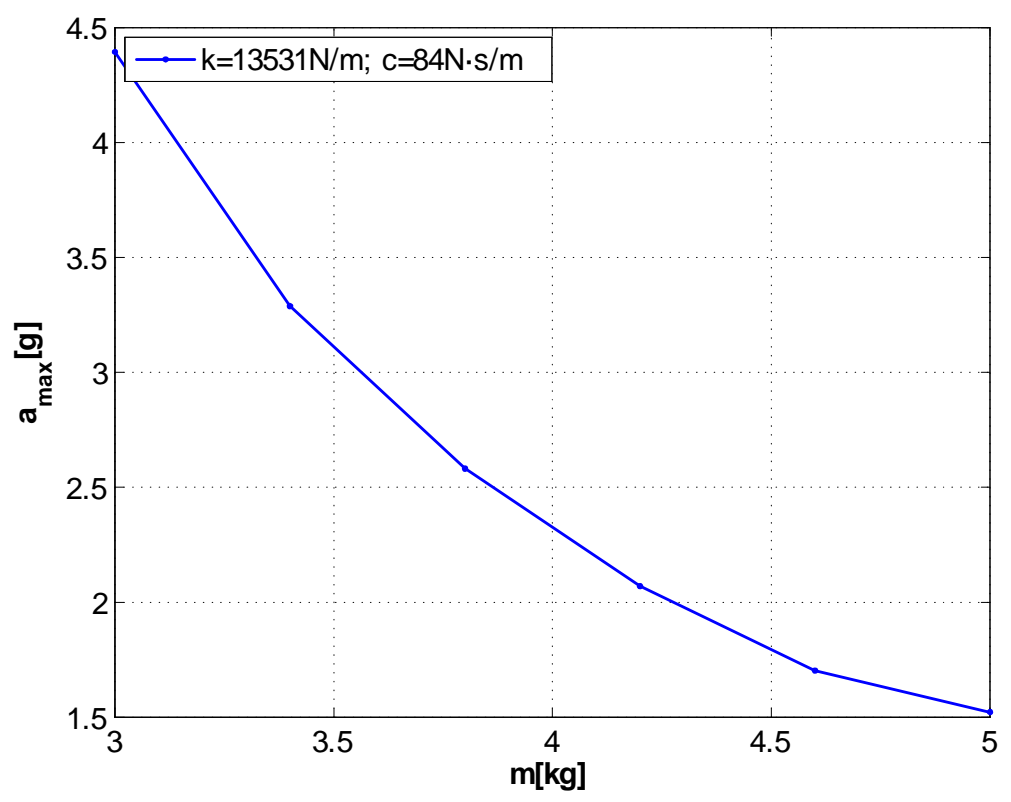

Figura 4-56: Análisis paramétrico en masa (truncatura 21 modos)

En cambio, el aspecto de la gráfica 4-58 es muy similar al caso calculado con 151 modos. 


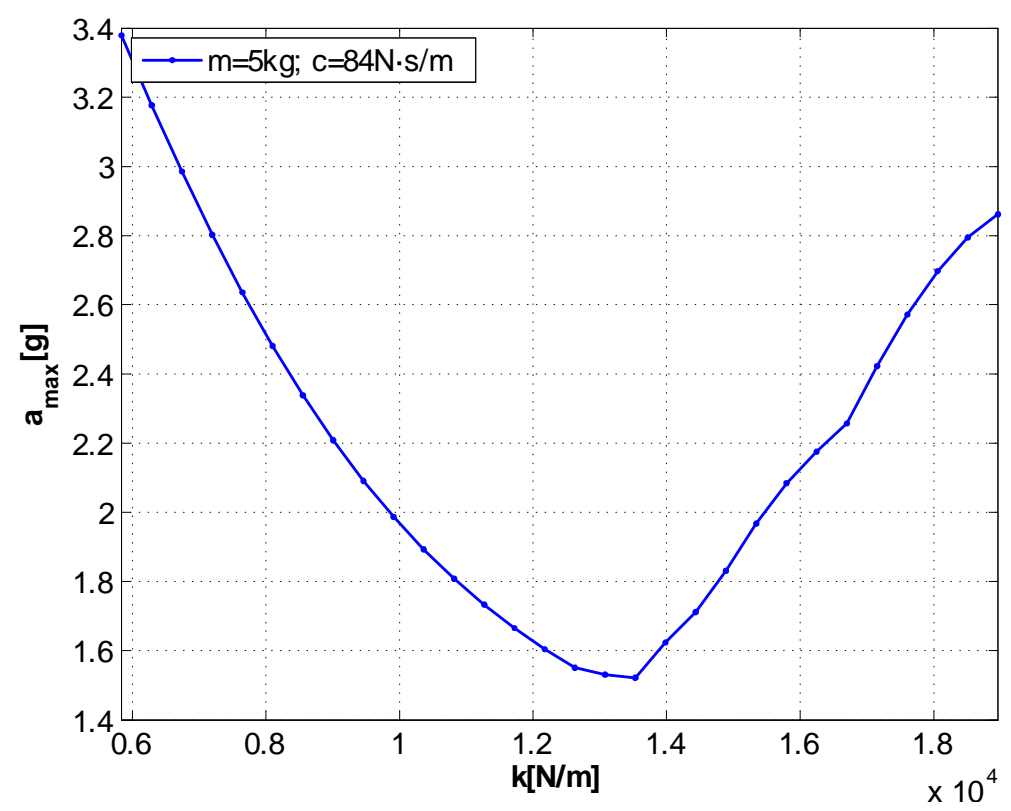

Figura 4-57: Análisis paramétrico en rigidez (truncatura 21 modos)

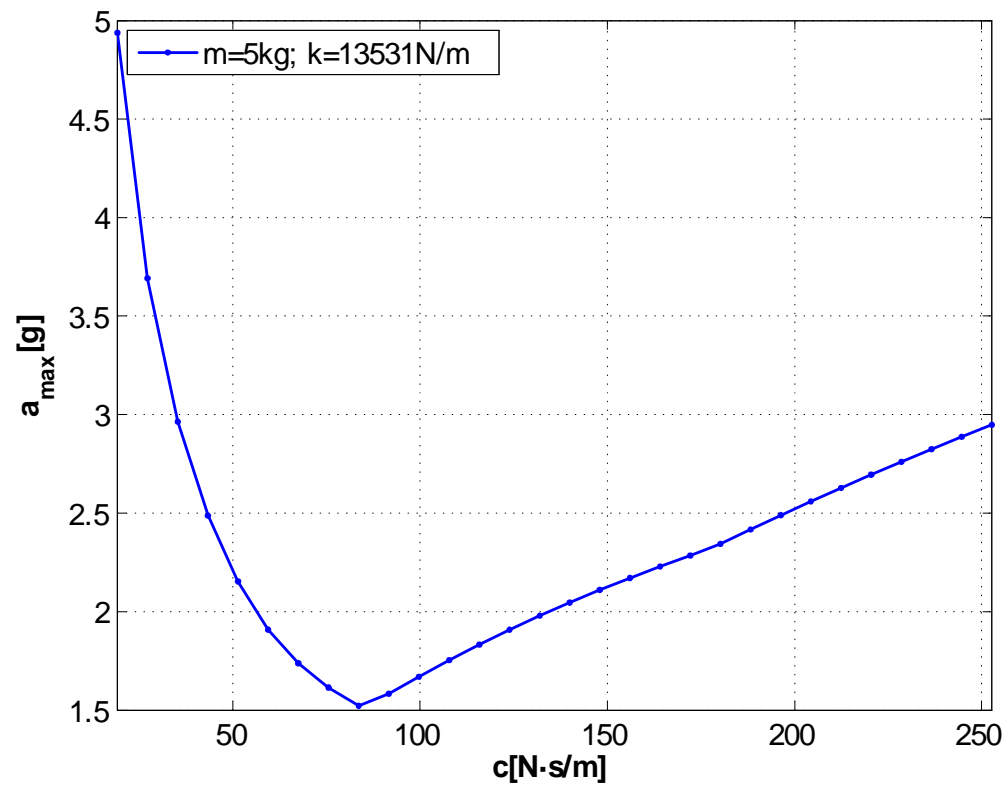

Figura 4-58: Análisis paramétrico en amortiguamiento (truncatura 21 modos) 
Las líneas isoparamétricas sitúan los mismos valores de mínimo y máximo de aceleraciones a pesar del cambio de curva óptimo (4-59)

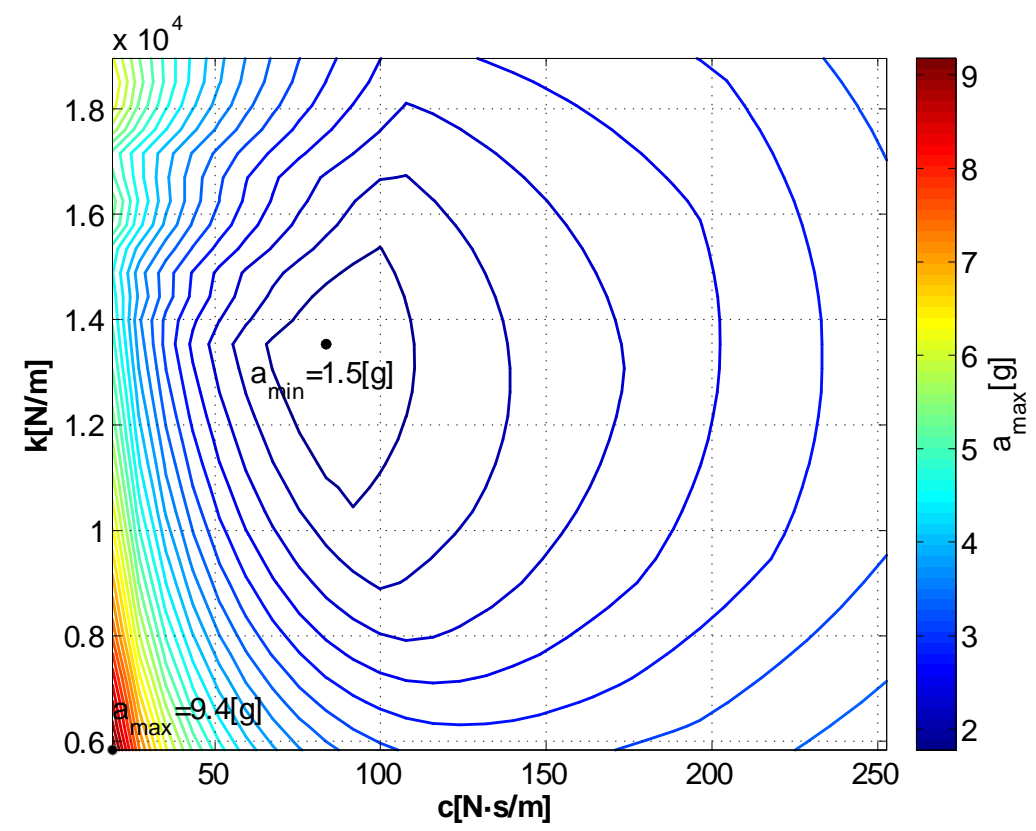

Figura 4-59: Gráfica de líneas isoparamétricas k-c (truncatura 21 modos)

En segundo lugar se muestran los resultados correspondientes a 60 modos. En la figura 4-60 se ofrece una muestra de los resultados de los 5400 análisis realizados de los que se extrae la curva óptima de atenuación de respuesta en frecuencia del equipo que para este caso se logran con una masa de $5 \mathrm{~kg}$, una rigidez de $13531 \mathrm{~N} / \mathrm{m}$ y un amortiguamiento de $84 \mathrm{Ns} / \mathrm{m}$. 


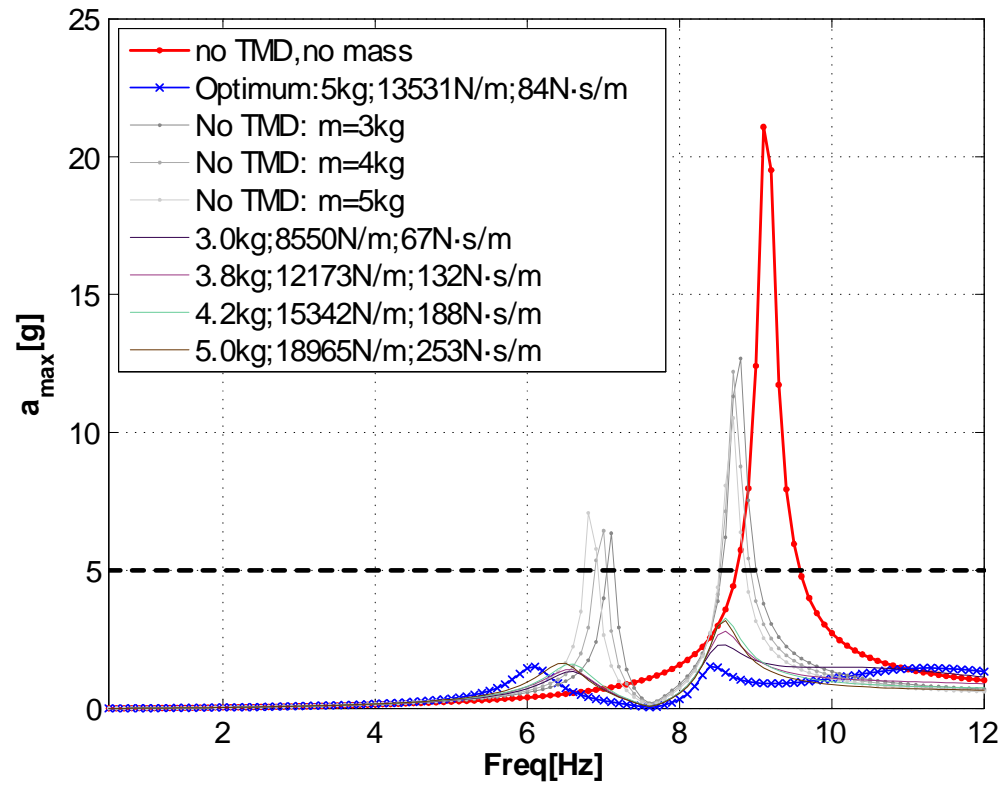

Figura 4-60: Análisis paramétrico. Resumen de respuesta en frecuencia de aceleraciones en punta de ala izquierda (truncatura 60 modos)

Las respuestas de aceleraciones verticales en la semiala izquierda son muy similares al caso con menor número de modos retenido. Se pueden ver en las figuras 4-61, 4-62 y 4-63. 


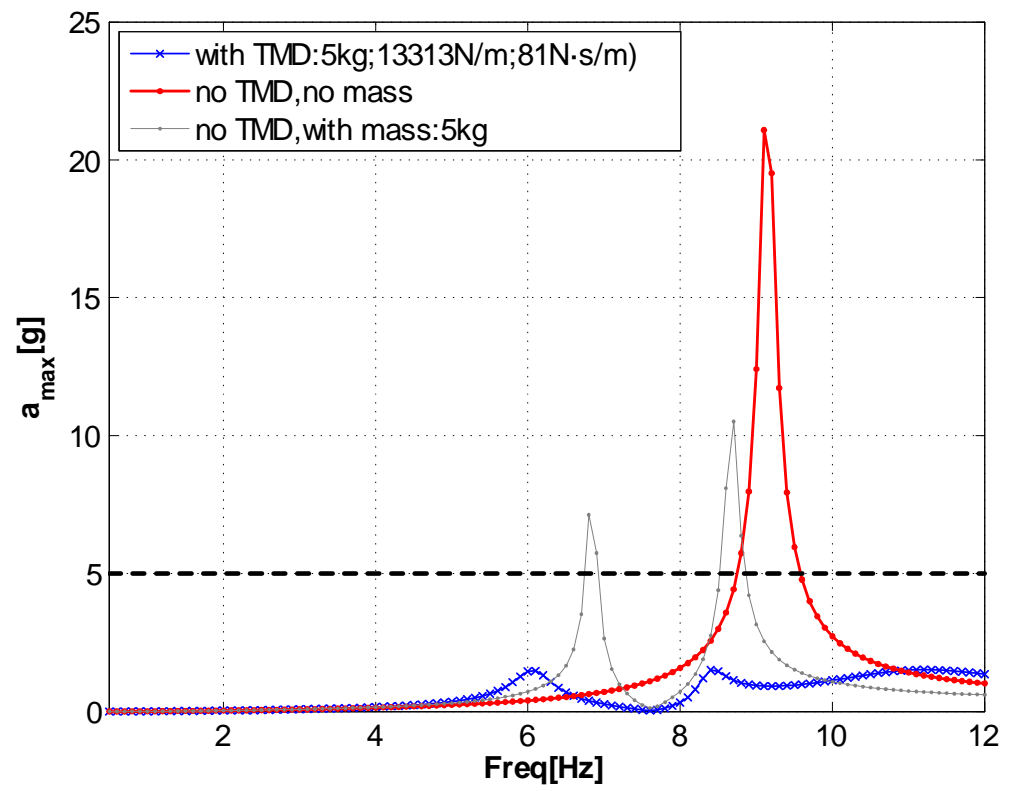

Figura 4-61: Respuesta en frecuencia de aceleraciones en punta de ala izquierda (truncatura 60 modos)

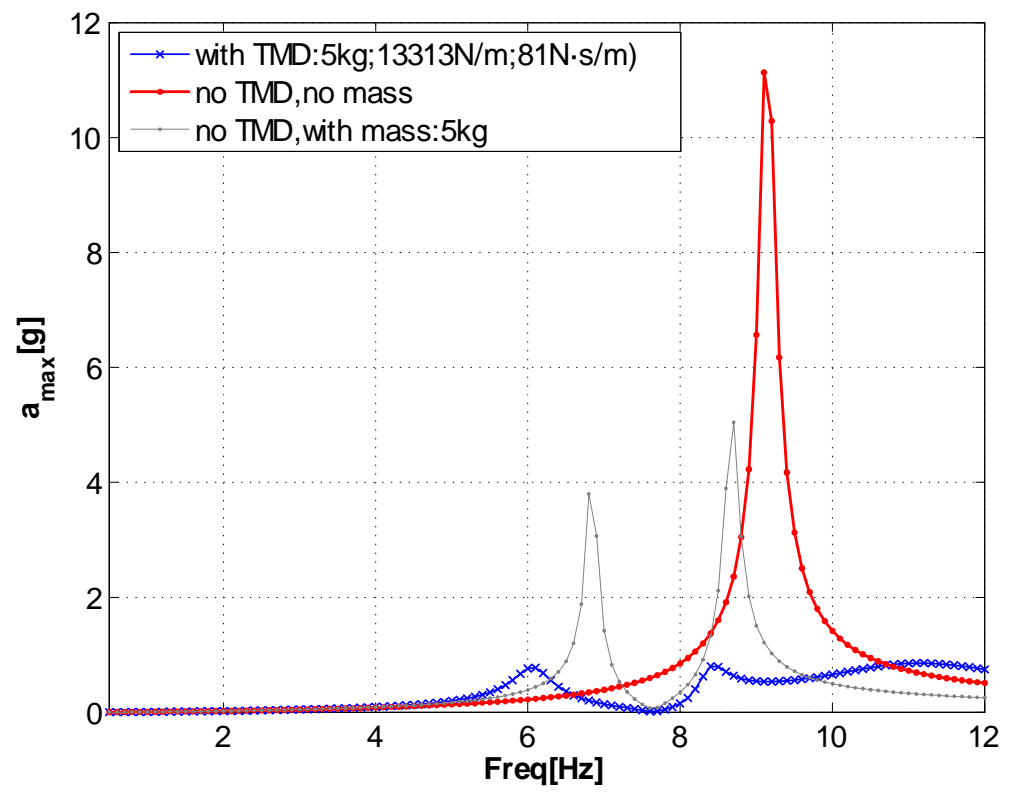

Figura 4-62: Respuesta en frecuencia de aceleraciones en inicio de estrechamiento en semiala izquierda (truncatura 60 modos) 


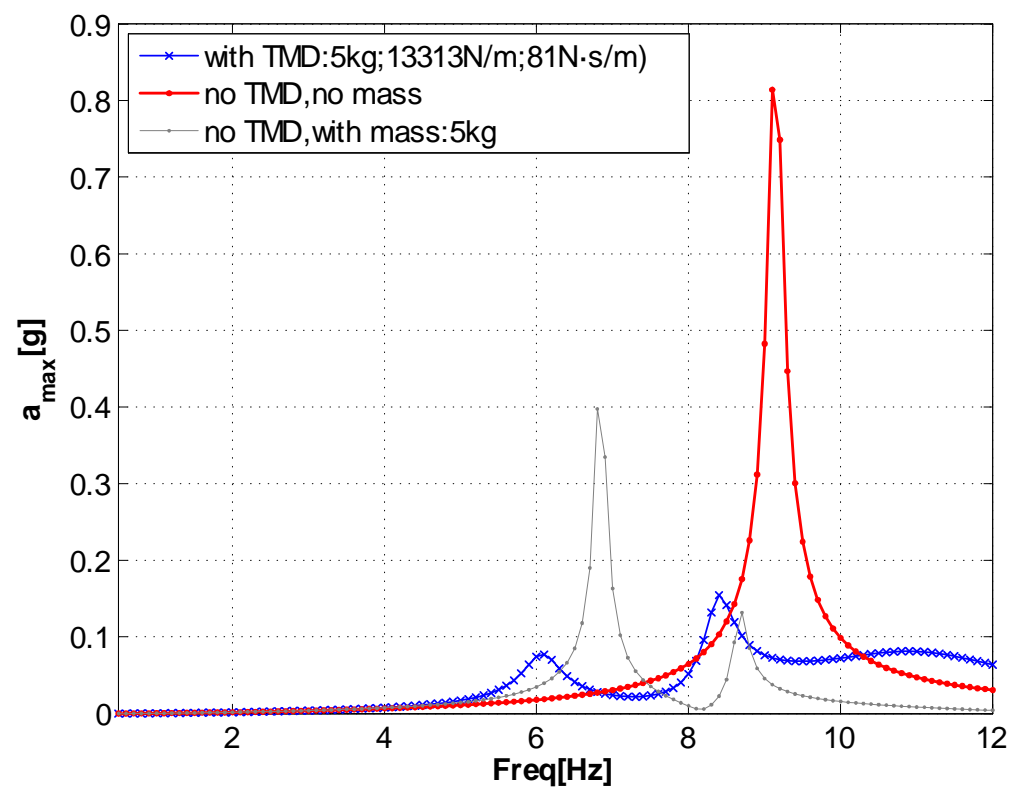

Figura 4-63: Respuesta en frecuencia de aceleraciones en encastre de semiala izquierda (truncatura 60 modos) 
Las figuras 4-64, 4-65 y 4-66 permiten identificar las diferencias de los extremos del intervalo para el caso de la truncatura realizada con 60 modos. Puede comprobarse que tanto la forma como los valores son muy similares a los logrados con la truncatura de 21 modos.

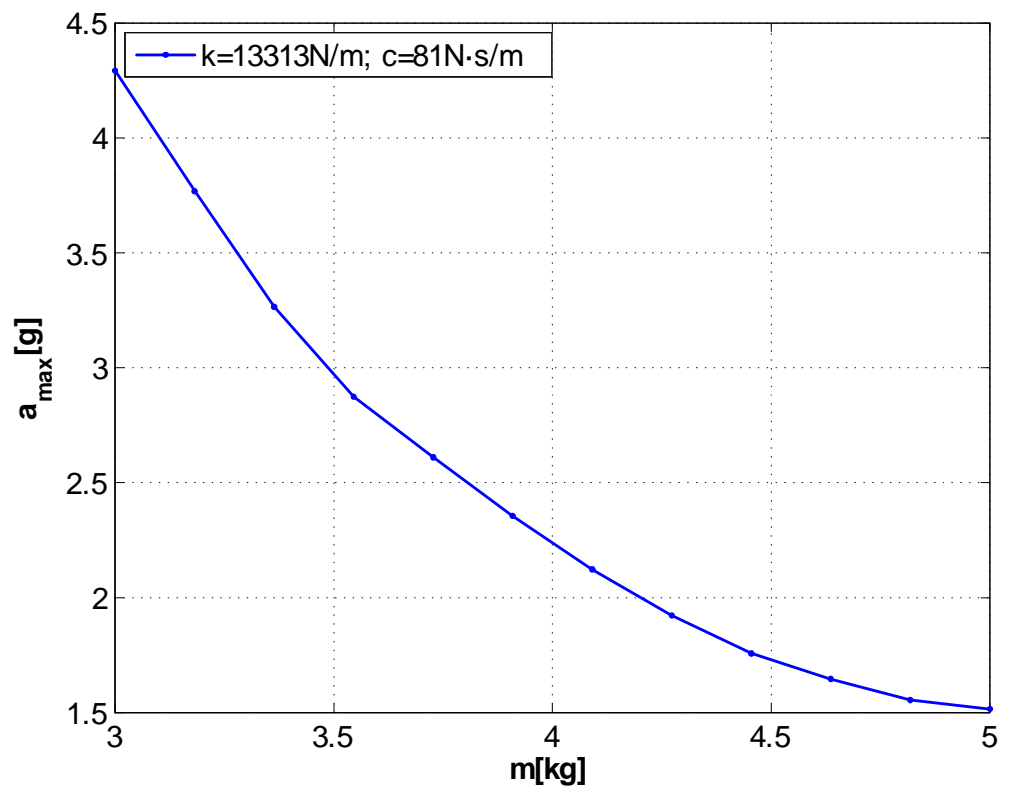

Figura 4-64: Análisis paramétrico en masa (truncatura 60 modos)

La gráfica de líneas isoparamétricas 4-67 ofrece también un resultado semejante al caso de 21 modos. 


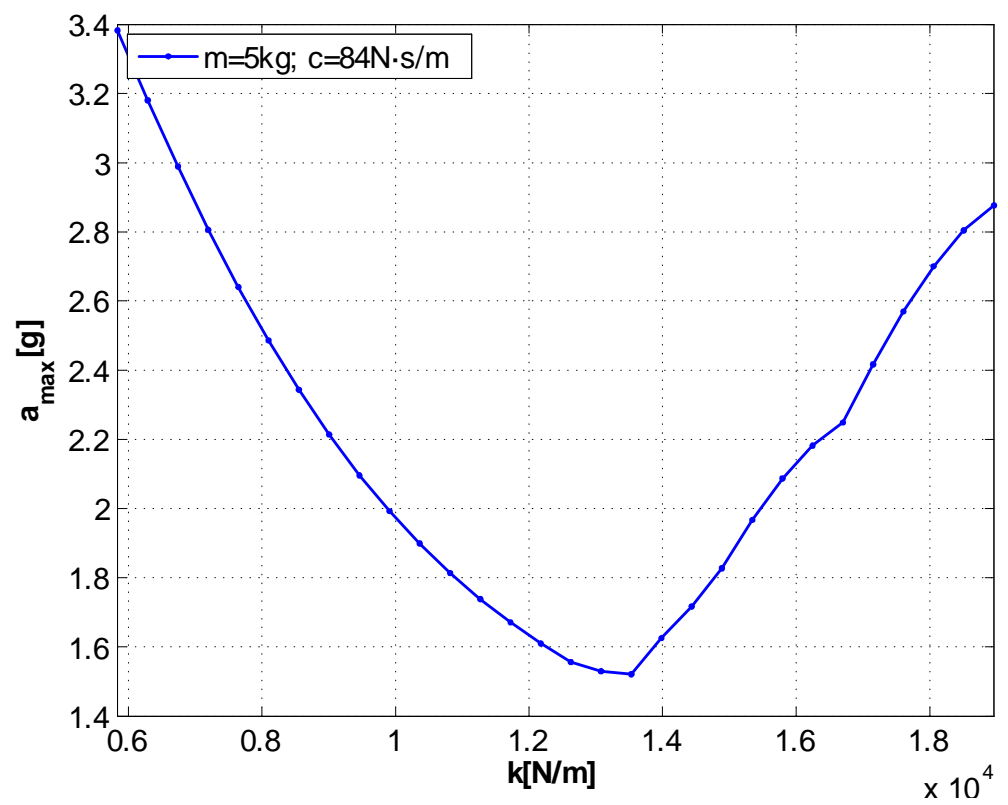

Figura 4-65: Análisis paramétrico en rigidez (truncatura 60 modos)

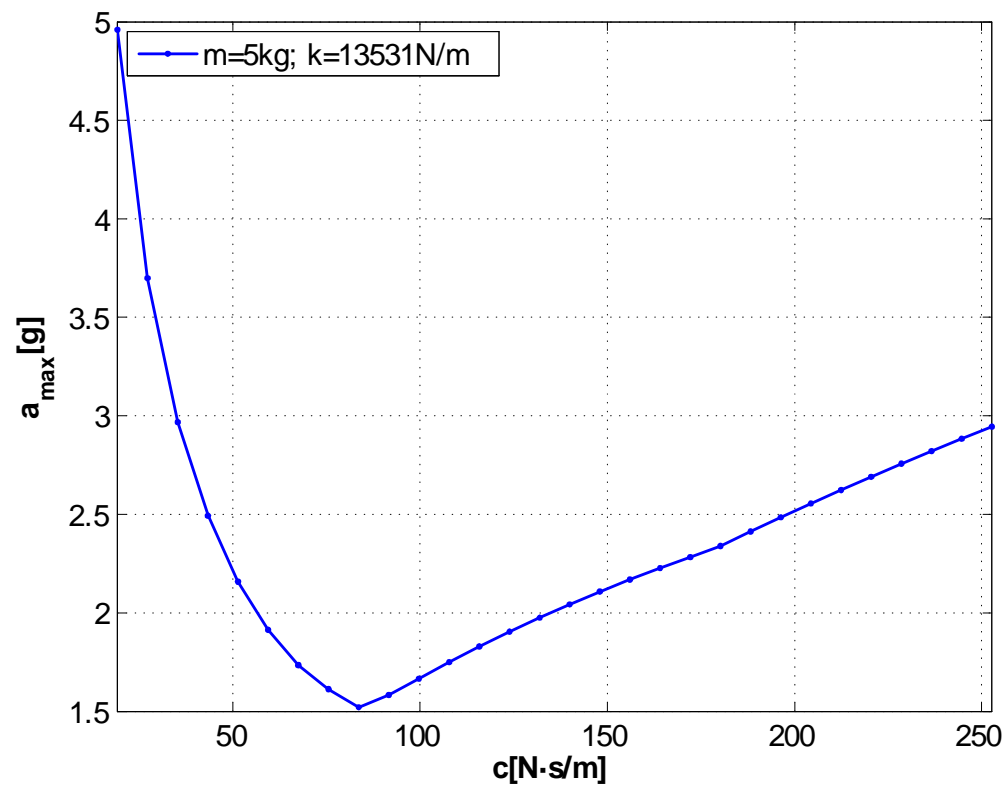

Figura 4-66: Análisis paramétrico en amortiguamiento (truncatura 60 modos) 


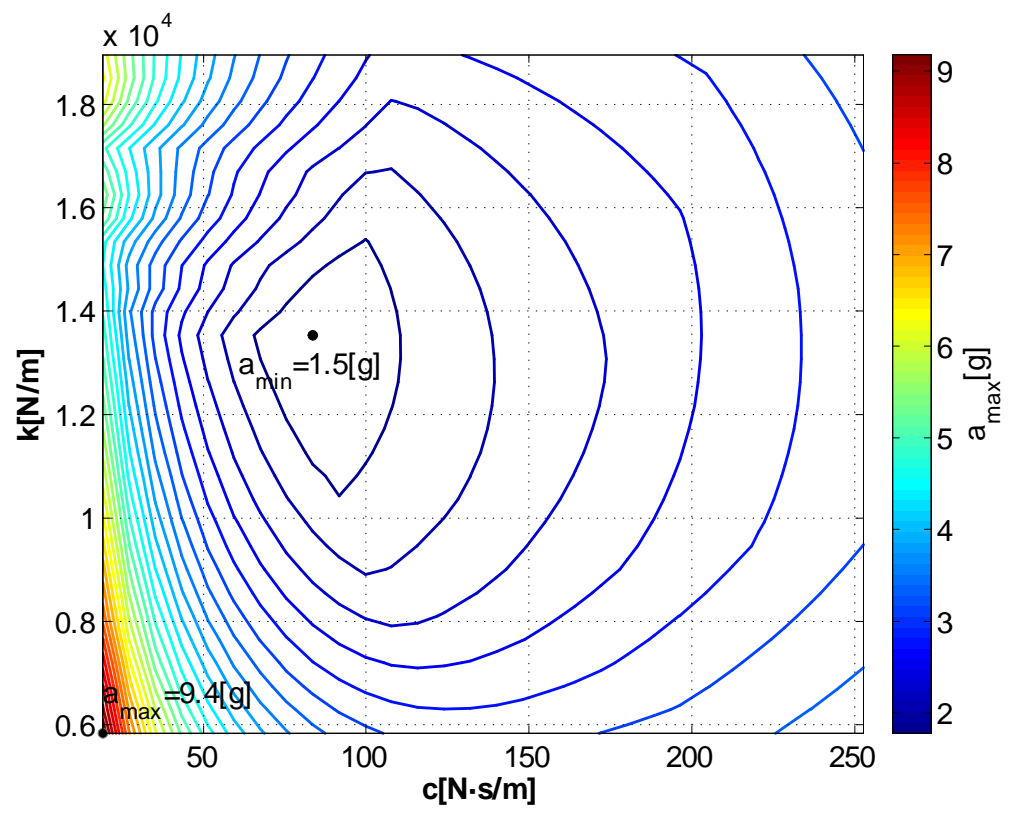

Figura 4-67: Gráfica de líneas isoparamétricas k-c (truncatura 60 modos) 
El tercer y último estudio comparativo se realiza con una truncatura modal en la que se retienen 100 modos de forma que se puedan contrastar adecuadamente sus resultados con el análisis de referencia de 151 modos y los anteriormente presentados con 21 y 60 modos.

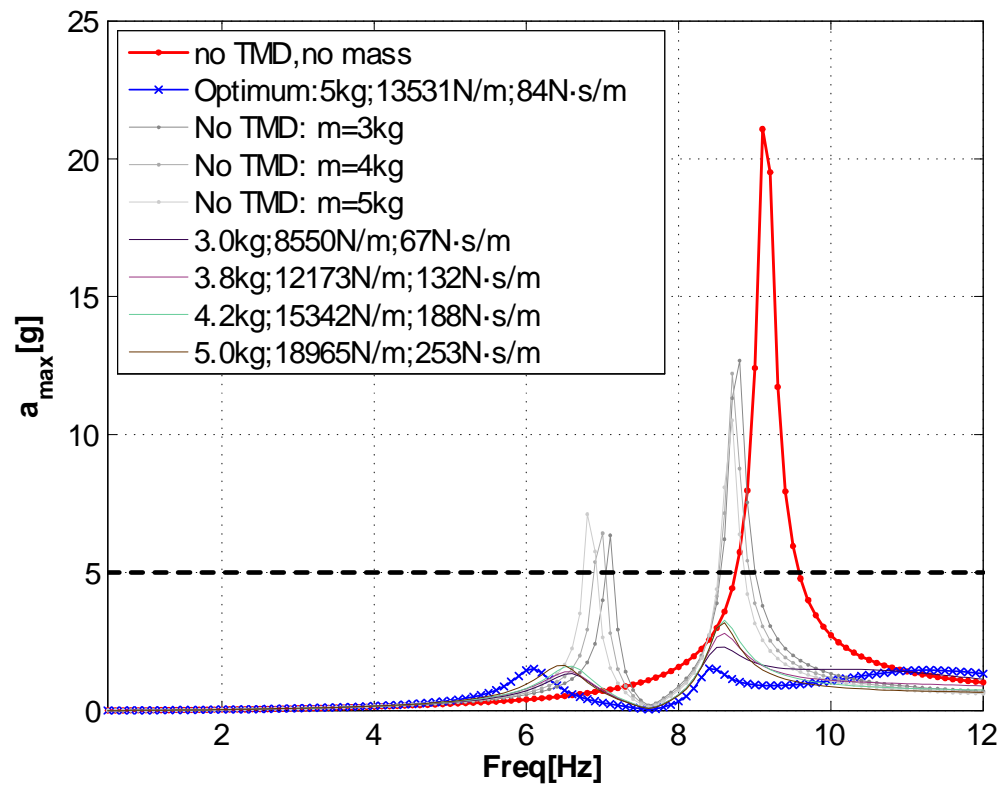

Figura 4-68: Análisis paramétrico. Resumen de respuesta en frecuencia de aceleraciones en punta de ala izquierda (truncatura 100 modos)

Puede comprobarse en la gráfica 4-68 que la respuesta de este caso tampoco difiere sustancialmente respecto a los anteriores. La mayor diferencia se localiza en el primer máximo a 6.8 $\mathrm{Hz}$ en el que la aceleración de este caso calculado con 100 modos en la truncatura difiere en $0.2 \mathrm{~m} / \mathrm{s}^{2}$ lo que supone una diferencia menor a un $1 \%$ respecto al caso calculado con 60 modos para el conjunto óptimo de parámetros del TMD. La curva de la aceleración de este óptimo se muestra también en la figura 4-69. Los valores de las propiedades del TMD son en cambio iguales. 


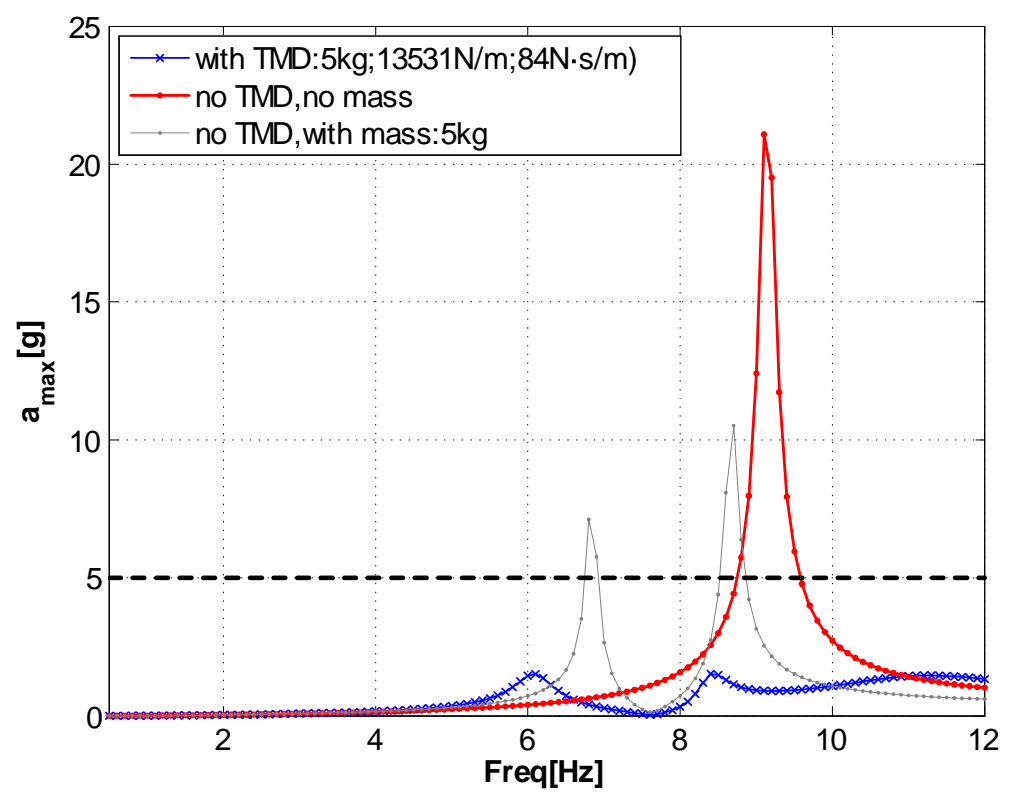

Figura 4-69: Respuesta en frecuencia de aceleraciones en punta de ala izquierda (truncatura 100 modos)

En las figuras 4-70 y 4-71 se muestran las aceleraciones verticales en el inicio del estrechamiento y en el encastre de la semiala izquierda para el caso citado de empleo de 100 modos propios. 


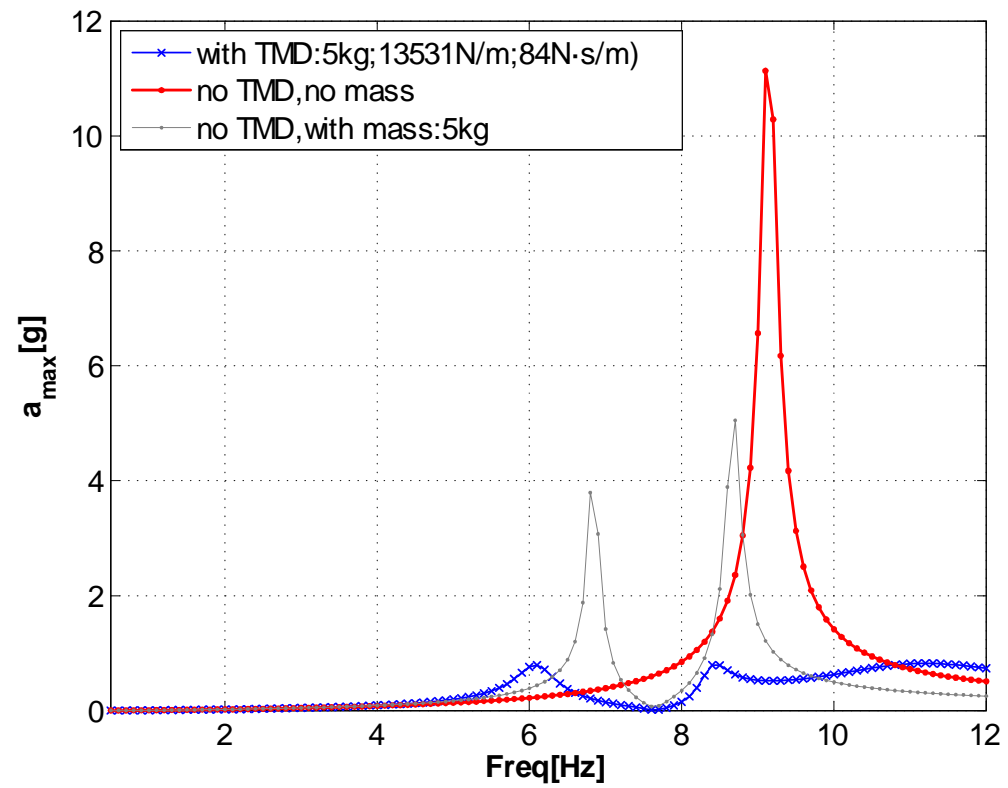

Figura 4-70: Respuesta en frecuencia de aceleraciones en inicio de estrechamiento en semiala izquierda (truncatura 100 modos)

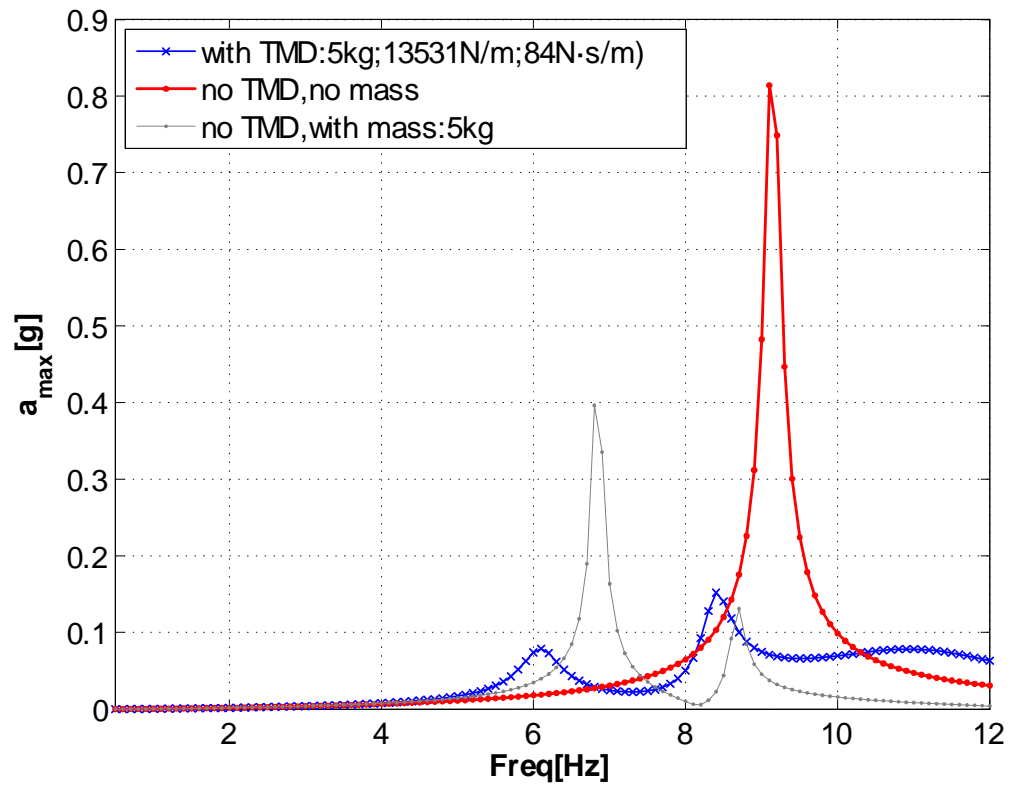

Figura 4-71: Respuesta en frecuencia de aceleraciones en encastre de semiala izquierda (truncatura 100 modos) 
Los análisis paramétricos en masa (figura 4-72), en rigidez (figura 4-73) y en amortiguamiento (figura 4-74) no presentan ninguna diferencia apreciable respecto al caso anterior.

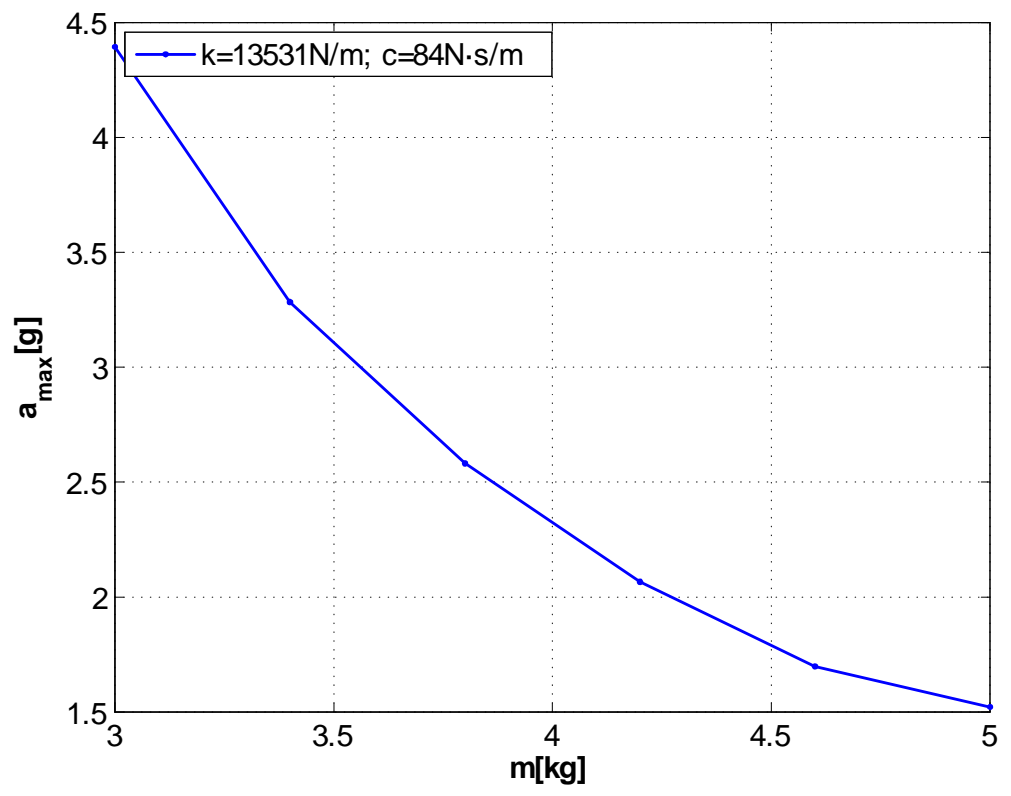

Figura 4-72: Análisis paramétrico en masa (truncatura 100 modos)

De forma análoga, la distribución del mínimo de los mínimos y del máximo de los máximos que se representan en el mapa de curvas de nivel (figura 4-75) tampoco sufre modificaciones. 


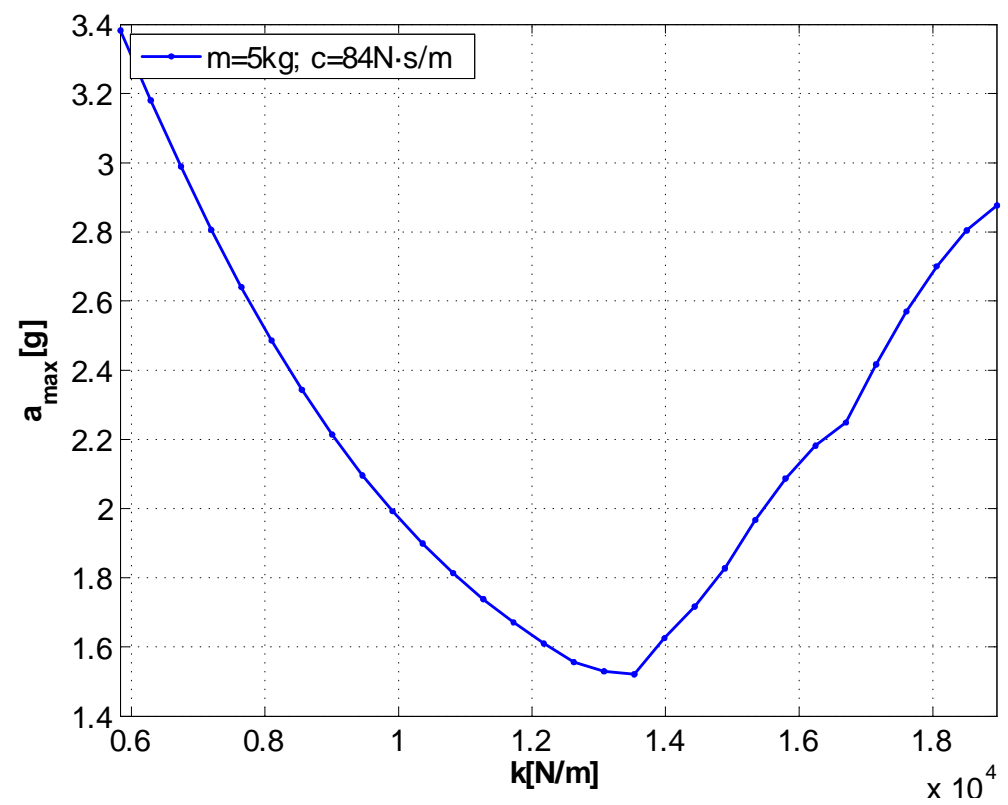

Figura 4-73: Análisis paramétrico en rigidez (truncatura 100 modos)

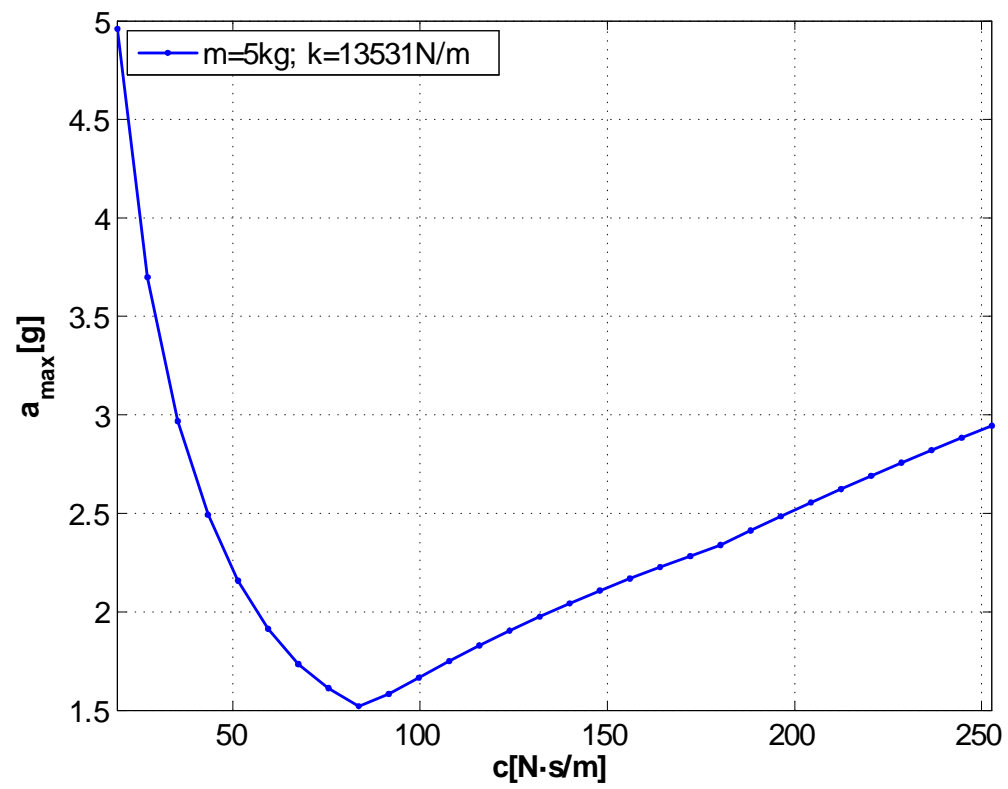

Figura 4-74: Análisis paramétrico en amortiguamiento (truncatura 100 modos) 


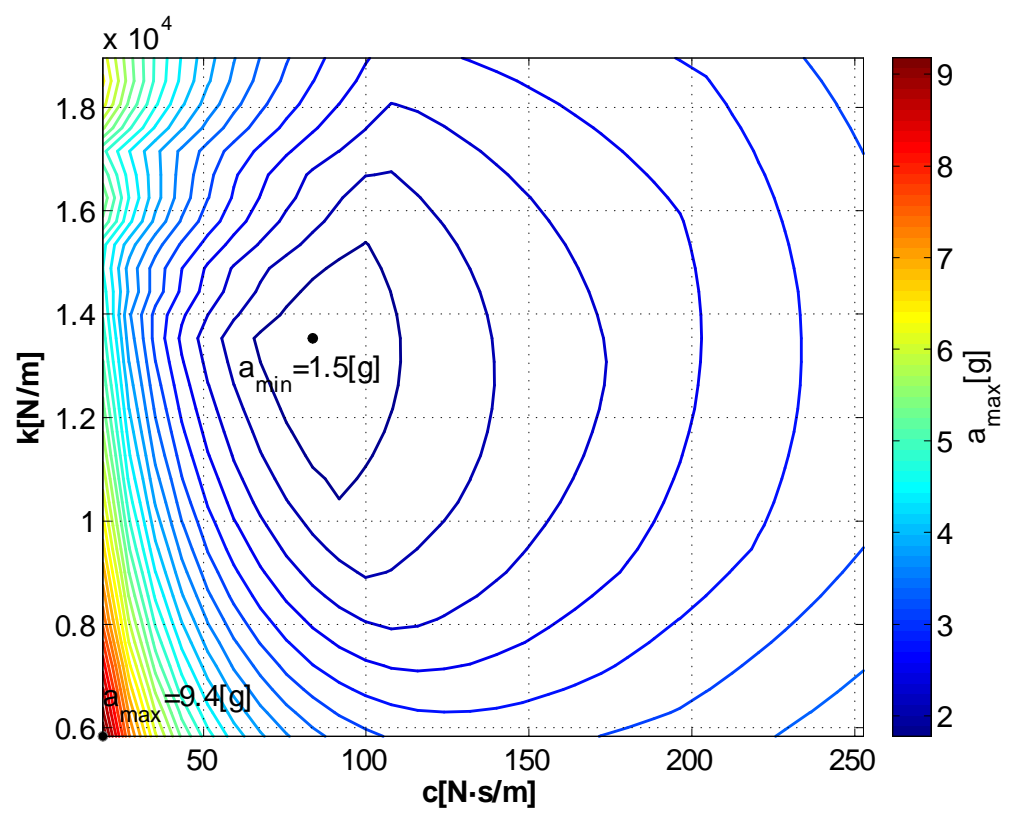

Figura 4-75: Gráfica de líneas isoparamétricas k-c (truncatura 100 modos) 
Los resultados comparativos con el método empleando 21, 60, 100 y 151 modos se muestran en la siguentes tablas, donde además se comparan con los resultados obtenidos en los análisis ejecutados mediante un programa comercial de elementos finitos. En la tabla 4.11 se comparan los resultados de la respuesta en frecuencia en punta de ala izquierda de aceleración vertical con el equipo añadido pero sin dispositivo de atenuación de la respuesta:

\begin{tabular}{|c|c|c|c|}
\hline Frecuencia $(\mathrm{Hz})$ & 6.8 & 7.6 & 8.7 \\
\hline A-Aceleración con método tesis con 21 modos $\left(\mathrm{m} / \mathrm{s}^{2}\right)$ & 68.8 & 1.3 & 103.3 \\
\hline B-Aceleración con método tesis con 60 modos $\left(\mathrm{m} / \mathrm{s}^{2}\right)$ & 69.5 & 1.3 & 103.2 \\
\hline C-Aceleración con método tesis con 100 modos $\left(\mathrm{m} / \mathrm{s}^{2}\right)$ & 69.7 & 1.3 & 103.2 \\
\hline D-Aceleración con método tesis con 151 modos $\left(\mathrm{m} / \mathrm{s}^{2}\right)$ & 69.9 & 1.3 & 103.1 \\
\hline E-Aceleración con programa comercial $\left(\mathrm{m} / \mathrm{s}^{2}\right)$ & 64.3 & 1.2 & 100.4 \\
\hline Diferencia A-E & $7 \%$ & $1 \%$ & $3 \%$ \\
\hline Diferencia B-E & $8 \%$ & $1 \%$ & $3 \%$ \\
\hline Diferencia C-E & $9 \%$ & $1 \%$ & $3 \%$ \\
\hline Diferencia D-E & $9 \%$ & $1 \%$ & $3 \%$ \\
\hline
\end{tabular}

Tabla 4.11: Comparación de máximos de aceleraciones con variación modos considerados sin TMD

Puede verse que las diferencias respecto a los valores obtenidos con el programa comercial son menores del $10 \%$ en todos los casos, siendo ligeramente más cercanos para el caso calculado con 21 modos. Las diferencias entre los distintos valores obtenidos con el método para diferentes número de modos son menores de un $2 \%$.

En la tabla 4.12 se comparan los resultados de la respuesta en frecuencia en punta de ala izquierda de aceleración vertical con el equipo añadido incluido el TMD.

En este caso, las respuestas difieren en algo más de un 10\% (hasta un 12\%) en el caso del máximo a $6.1 \mathrm{~Hz}$ mientras que los valores en el máximo a $8.4 \mathrm{~Hz}$ y el mínimo a $7.6 \mathrm{~Hz}$ son más cercanos al valor obtenido sin emplear el método de aceleración de análisis. Las diferencias entre los valores obtenidos con el método son menores de un $3 \%$.

En la última tabla se comparan los resultados de la respuesta en frecuencia en punta de ala izquierda de aceleración vertical con los resultados obtenidos en los análisis transitorios 


\begin{tabular}{|c|c|c|c|}
\hline Frecuencia $(\mathrm{Hz})$ & 6.1 & 7.6 & 8.4 \\
\hline A-Aceleración con método tesis con 21 modos $\left(\mathrm{m} / \mathrm{s}^{2}\right)$ & 14.8 & 0.4 & 14.9 \\
\hline B-Aceleración con método tesis con 60 modos $\left(\mathrm{m} / \mathrm{s}^{2}\right)$ & 14.8 & 0.4 & 14.9 \\
\hline C-Aceleración con método tesis con 100 modos $\left(\mathrm{m} / \mathrm{s}^{2}\right)$ & 14.8 & 0.4 & 14.9 \\
\hline D-Aceleración con método tesis con 151 modos $\left(\mathrm{m} / \mathrm{s}^{2}\right)$ & 14.5 & 0.4 & 14.7 \\
\hline E-Aceleración con programa comercial $\left(\mathrm{m} / \mathrm{s}^{2}\right)$ & 13.2 & 0.4 & 15.1 \\
\hline Diferencia A-E & $12 \%$ & $0 \%$ & $1 \%$ \\
\hline Diferencia B-E & $12 \%$ & $0 \%$ & $1 \%$ \\
\hline Diferencia C-E & $12 \%$ & $0 \%$ & $1 \%$ \\
\hline Diferencia D-E & $10 \%$ & $3 \%$ & $2 \%$ \\
\hline
\end{tabular}

Tabla 4.12: Comparación de máximos de aceleraciones con variación modos considerados con TMD

ejecutados sobre el modelo completo con el código de análisis comercial.

\begin{tabular}{|c|c|c|c|}
\hline Frecuencia $(\mathrm{Hz})$ & 6.1 & 7.6 & 8.1 \\
\hline A-Aceleración con método tesis con 21 modos $\left(\mathrm{m} / \mathrm{s}^{2}\right)$ & 14.8 & 0.4 & 14.9 \\
\hline B-Aceleración con método tesis con 60 modos $\left(\mathrm{m} / \mathrm{s}^{2}\right)$ & 14.8 & 0.4 & 14.9 \\
\hline C-Aceleración con método tesis con 100 modos $\left(\mathrm{m} / \mathrm{s}^{2}\right)$ & 14.8 & 0.4 & 14.9 \\
\hline D-Aceleración con método tesis con 151 modos $\left(\mathrm{m} / \mathrm{s}^{2}\right)$ & 14.5 & 0.4 & 14.7 \\
\hline E-Aceleración con programa comercial $\left(\mathrm{m} / \mathrm{s}^{2}\right)$ & 12.8 & 0.4 & 14.3 \\
\hline Diferencia A-E & $16 \%$ & $10 \%$ & $4 \%$ \\
\hline Diferencia B-E & $16 \%$ & $10 \%$ & $4 \%$ \\
\hline Diferencia C-E & $16 \%$ & $10 \%$ & $4 \%$ \\
\hline Diferencia D-E & $13 \%$ & $7 \%$ & $3 \%$ \\
\hline
\end{tabular}

Tabla 4.13: Comparación de máximos de aceleraciones con análisis transitorio con variación modos considerados

Puede verse que los valores obtenidos para 21, 60, 100 y 151 modos retenidos son muy similares entre sí y diferentes respecto a los valores obtenidos con el programa de elementos finitos, un $16 \%$ en el caso del primer máximo, $10 \%$ para el mínimo y $4 \%$ para el segundo máximo. La retención de 151 modos proporciona resultados más cercanos a los obtenidos del modelo completo de elementos finitos.

Como se ha comentado, en el caso de referencia, para obtener los mejores resultados se han considerado los modos propios dentro del rango de frecuencias 0-250 Hz que son los 151 primeros. 


\subsection{Precisión por el barrido en frecuencia}

Uno de las posibles fuentes de pérdida de precisión puede ser el número de puntos con el que se determinan los máximos y mínimos de la respuesta en frecuencia. Si los extremos son muy acusados el paso, o distancia entre dos frecuencias consecutivas puede influir en la identificación de los puntos. Para analizar este efecto se han realizado análisis con 55, 116 y 231 frecuencias en el intervalo entre $0.5 \mathrm{~Hz}$ y $12 \mathrm{~Hz}$ con 5400 variaciones para cada uno de ellos de los parámetros de masa, rigidez y amortiguamiento del dispositivo de atenuación.

En el caso del barrido de menor densidad, se obtienen los resultados resumidos en la figura 4-76.

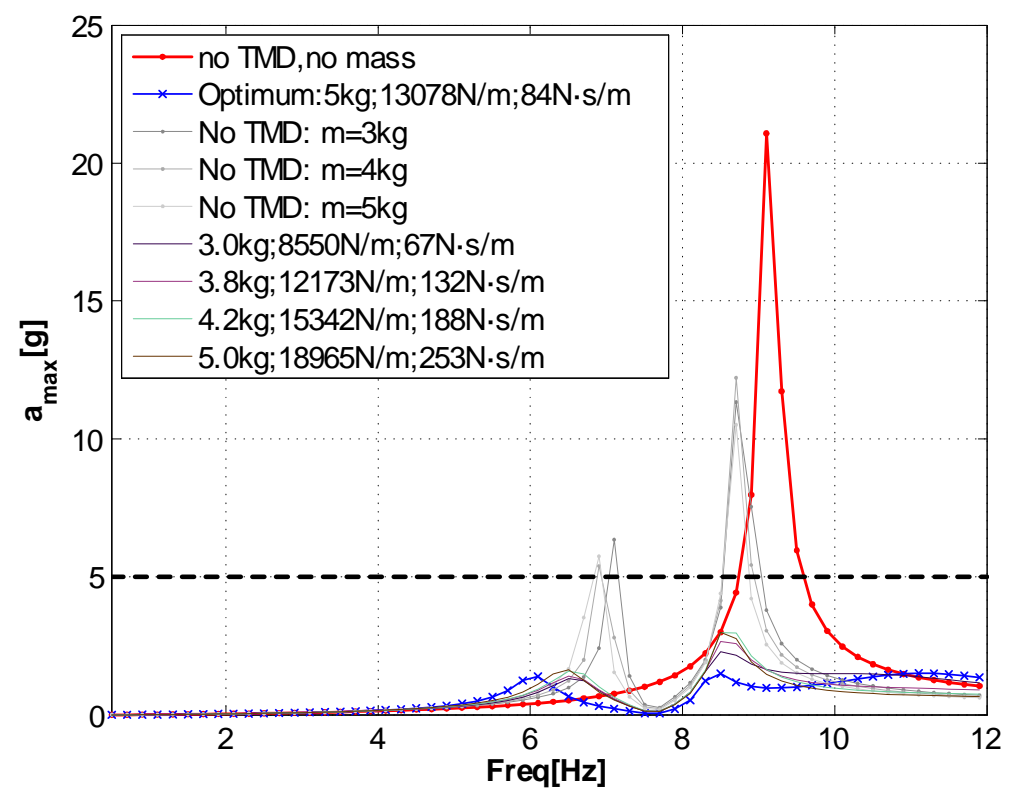

Figura 4-76: Análisis paramétrico. Resumen de respuesta en frecuencia de aceleraciones en punta de ala izquierda (barrido 55 frecuencias)

Puede comprobarse que la combinación óptima de parámetros se logra para:

- Masa del equipo incluyendo su anclaje: $5 \mathrm{~kg}$

- Rigidez del anclaje: 13.078 N/m

- Amortiguamiento de la unión: 84 Ns/m 
En las figuras 4-77, 4-78 y 4-79 se puede ver la respuesta en frecuencia de la aceleración vertical en tres puntos del ala en la que destaca la menor cantidad de puntos con los que se trazan las curvas. Dada la forma de las curvas en el entorno de los extremos, en particular de los máximos del rango de estudio, puede ser que la detección de dichos valores pueda verse influida por el paso de frecuencia.

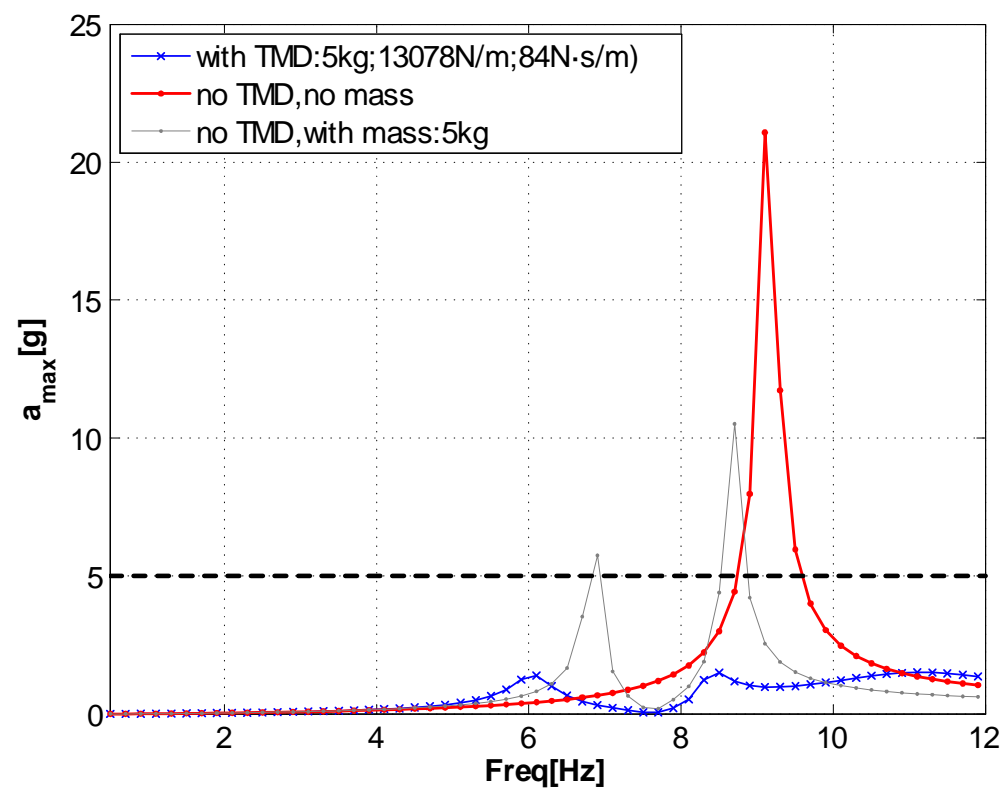

Figura 4-77: Respuesta en frecuencia de aceleraciones en punta de ala izquierda (barrido 55 frecuencias)

Debe destacarse que los extremos para este caso se localizan en frecuencias $(6.1 \mathrm{~Hz}, 7.5 \mathrm{~Hz}$ y $8.5 \mathrm{~Hz}$ para la curva que incluye el TMD y $6.9 \mathrm{~Hz}, 7.7 \mathrm{~Hz}$ y $8.9 \mathrm{~Hz}$ para el caso sin TMD) ligeramente distintas a las del caso nominal.

En los análisis paramétricos se detectan diferencias respecto al análisis de referencia realizado con un barrido a 231 frecuencias. Tal y como se puede ver en la figura 4-81 tanto la forma de la curva a partir del mínimo como su forma en el propio entorno del mínimo presenta cierta disparidad con las gráficas de referencia. 


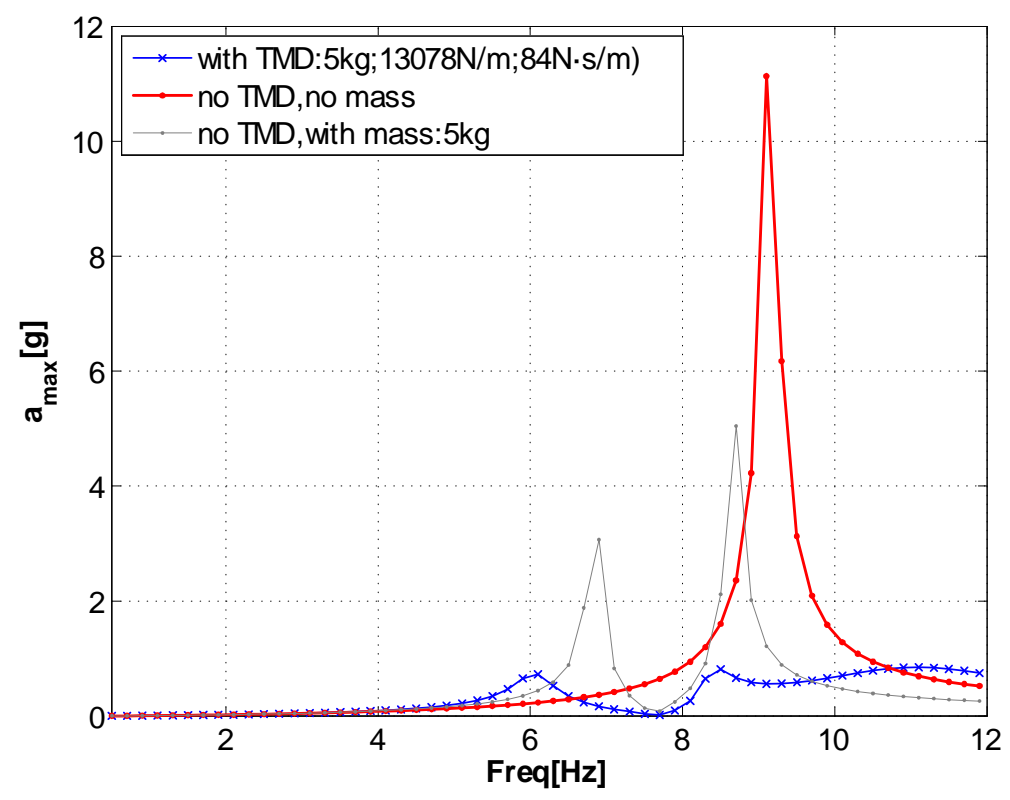

Figura 4-78: Respuesta en frecuencia de aceleraciones en inicio de estrechamiento en semiala izquierda (barrido 55 frecuencias)

Los cambios en la forma de las curvas de los análisis paramétricos, como el que se produce en el entorno de rigidez de $1.6 \cdot 10^{4} \mathrm{~N} / \mathrm{m}$, se deben a las diferencias en los valores de las aceleraciones no al número de puntos de estas figuras que dependen del número de parametrizaciones realizadas: 6 en masa y 30 tanto en rigidez como en amortiguamiento.

De forma análoga, en la gráfica 4-83, las líneas isoparamétricas de rigidez y amortiguamiento muestran diferencias en el comportamiento a alta rigidez y bajo amortiguamiento respecto al análisis de referencia con 116 puntos en el intervalo de estudio. 


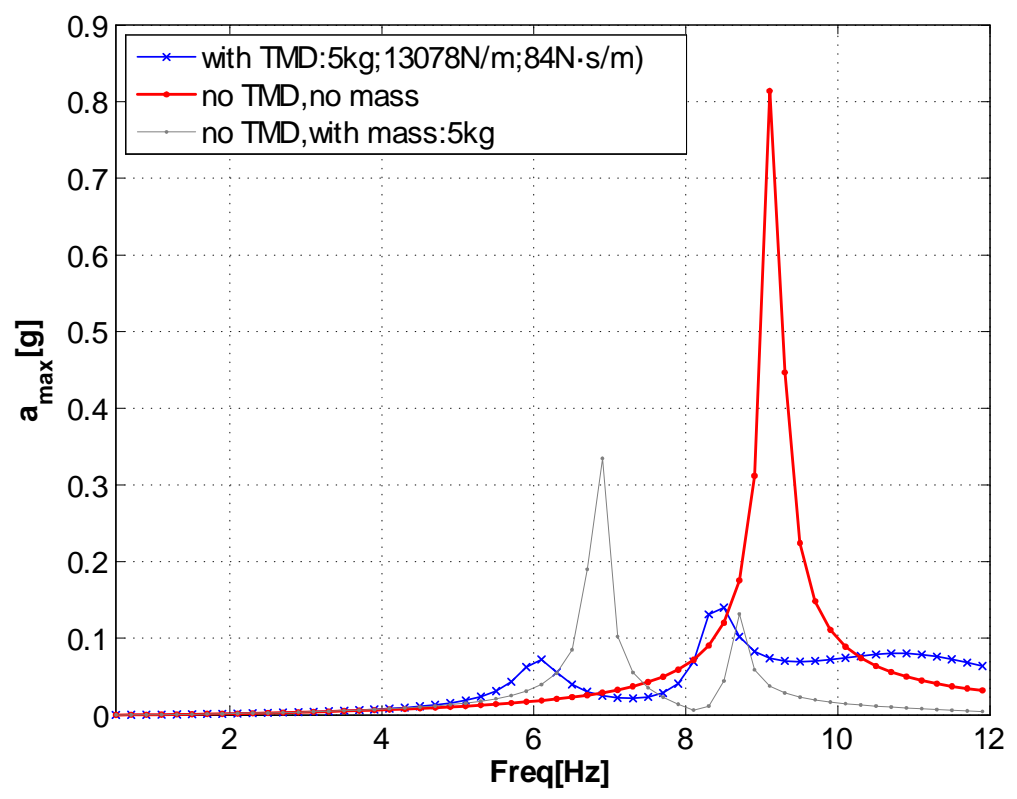

Figura 4-79: Respuesta en frecuencia de aceleraciones en encastre de semiala izquierda (barrido 55 frecuencias)

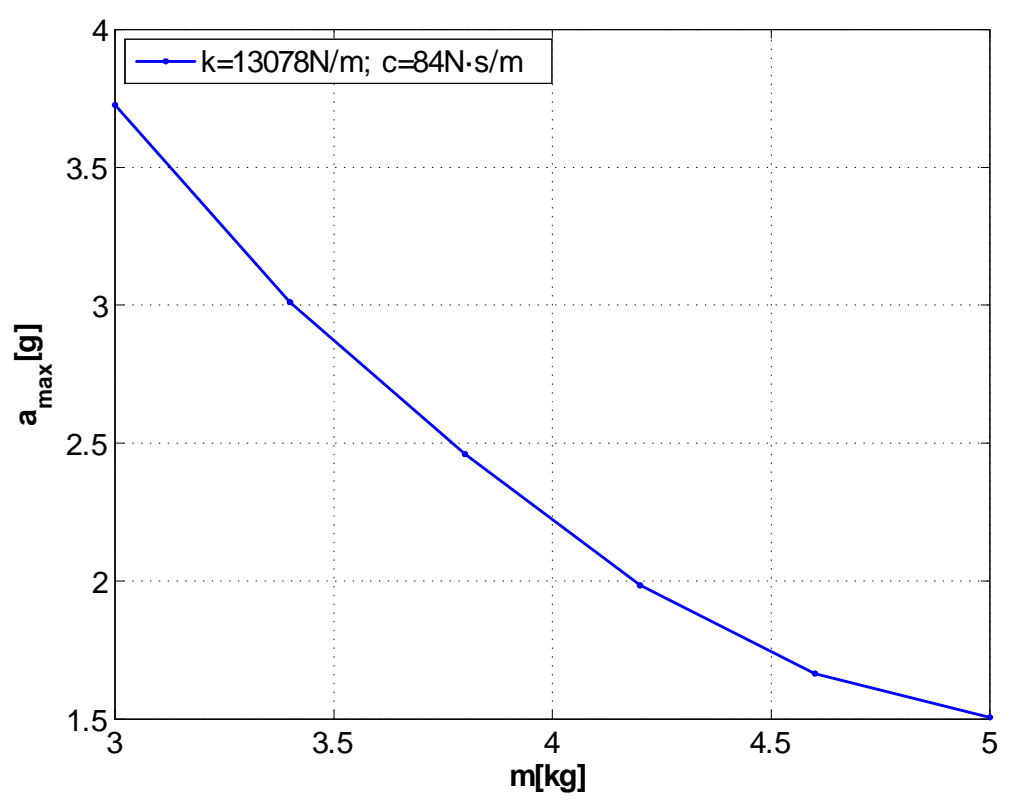

Figura 4-80: Análisis paramétrico en masa (barrido 55 frecuencias) 


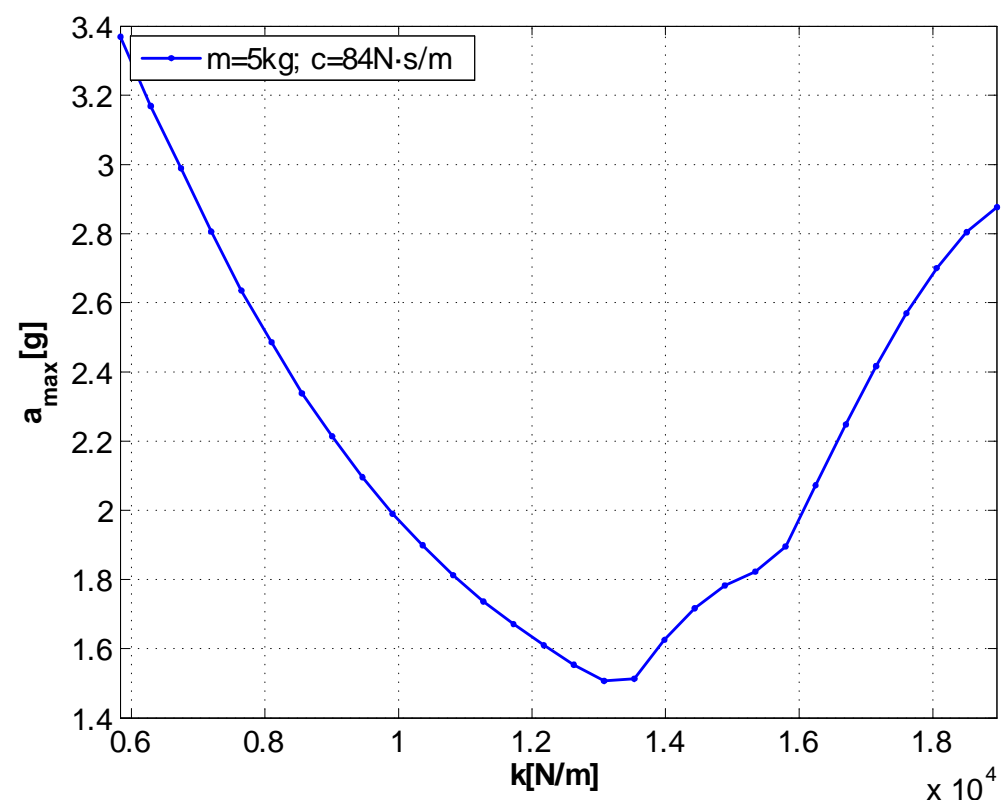

Figura 4-81: Análisis paramétrico en rigidez (barrido 55 frecuencias)

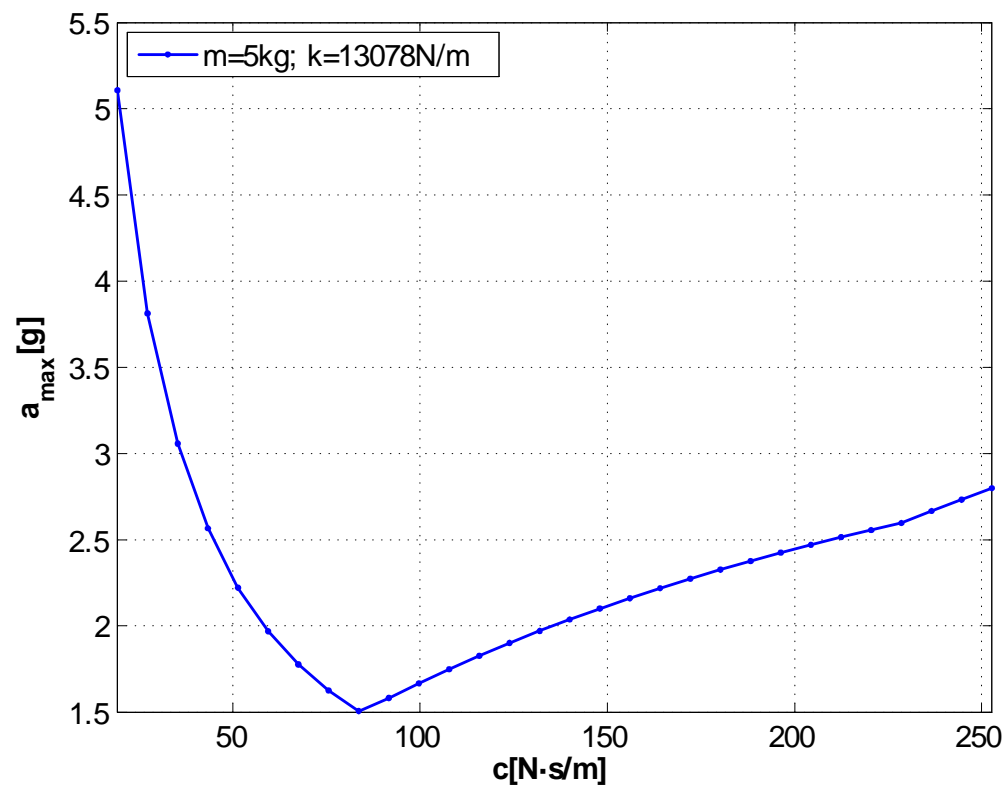

Figura 4-82: Análisis paramétrico en amortiguamiento (barrido 55 frecuencias) 


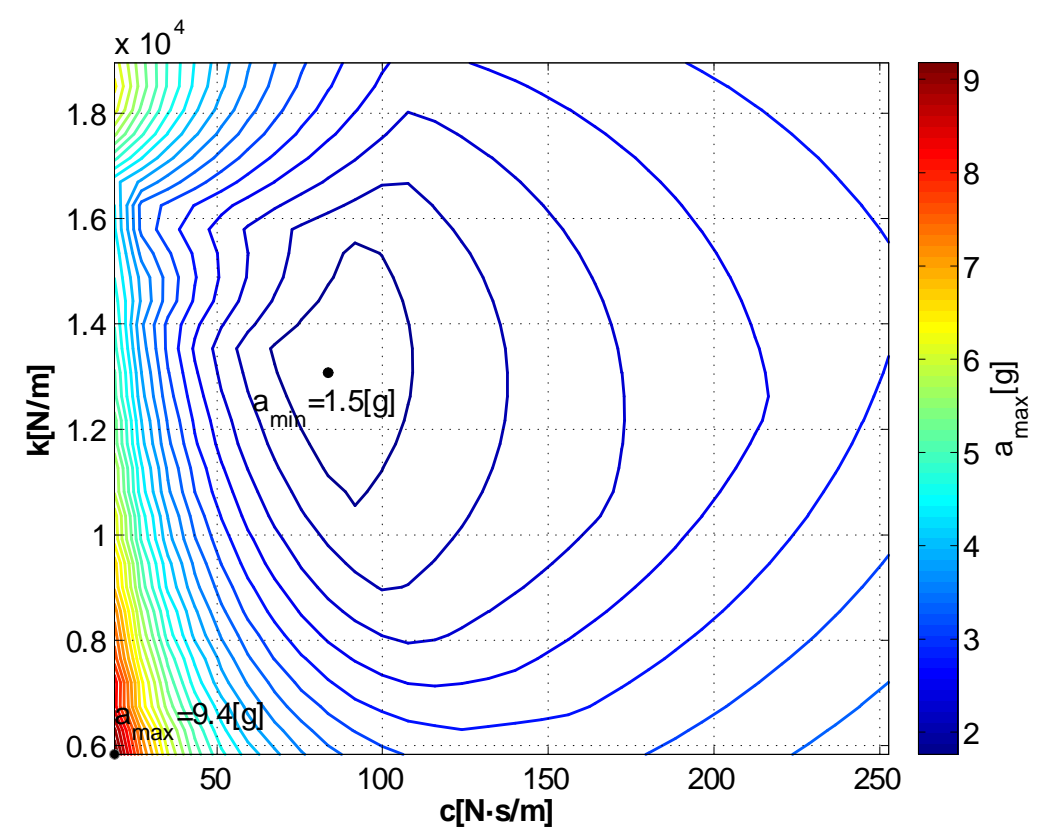

Figura 4-83: Gráfica de líneas isoparamétricas k-c (barrido 55 frecuencias) 
A continuación se incluyen los resultados obtenidos para un barrido de frecuencias superior al nominal, en concreto de 231 frecuencias en el rango entre $0.5 \mathrm{~Hz}$ y $12 \mathrm{~Hz}$. Puede comprobarse que la combinación óptima de parámetros del TMD en este caso se logra para:

- Masa del equipo incluyendo su anclaje: $5 \mathrm{~kg}$

- Rigidez del anclaje: $13.531 \mathrm{~N} / \mathrm{m}$

- Amortiguamiento de la unión: 84 Ns/m

Siendo dicha combinación semejante a la del caso de referencia ejecutado con 116 pasos en frecuencia.

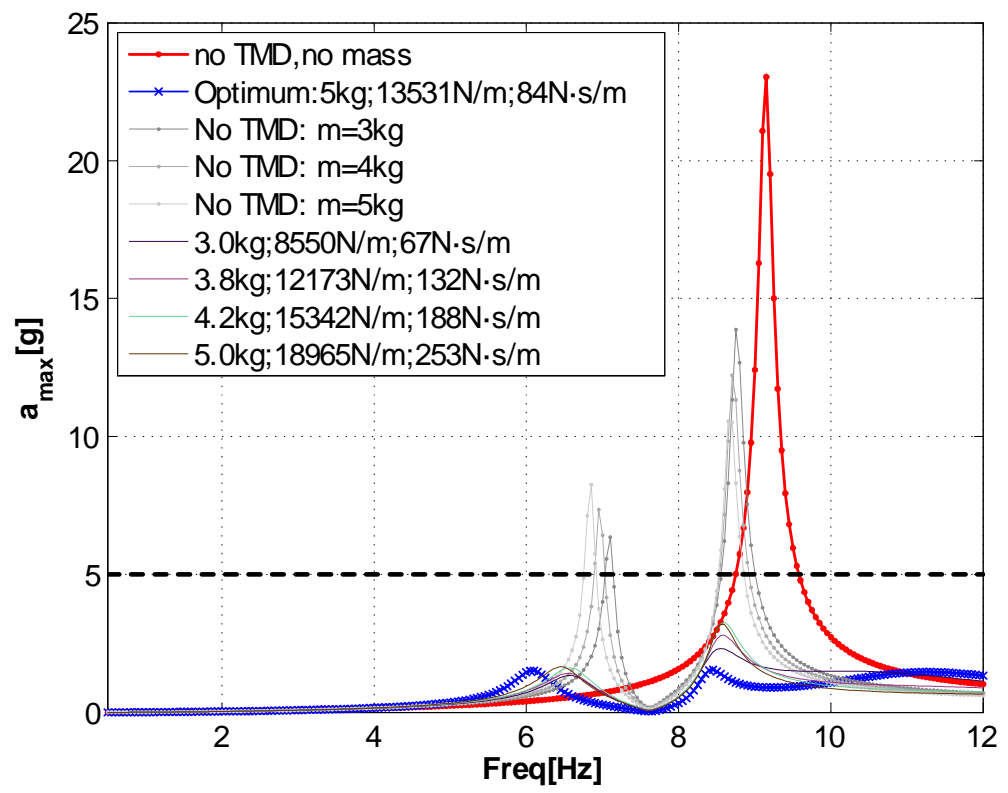

Figura 4-84: Análisis paramétrico. Resumen de respuesta en frecuencia de aceleraciones en punta de ala (barrido de frecuencias incrementado)

En las figuras 4-85, 4-86 y 4-87 se puede ver la respuesta en frecuencia de la aceleración vertical en tres puntos del ala en la que destaca la mayor cantidad de datos con los que se trazan las curvas que previsiblemente pudiera tener influencia en la detección de los extremos. 


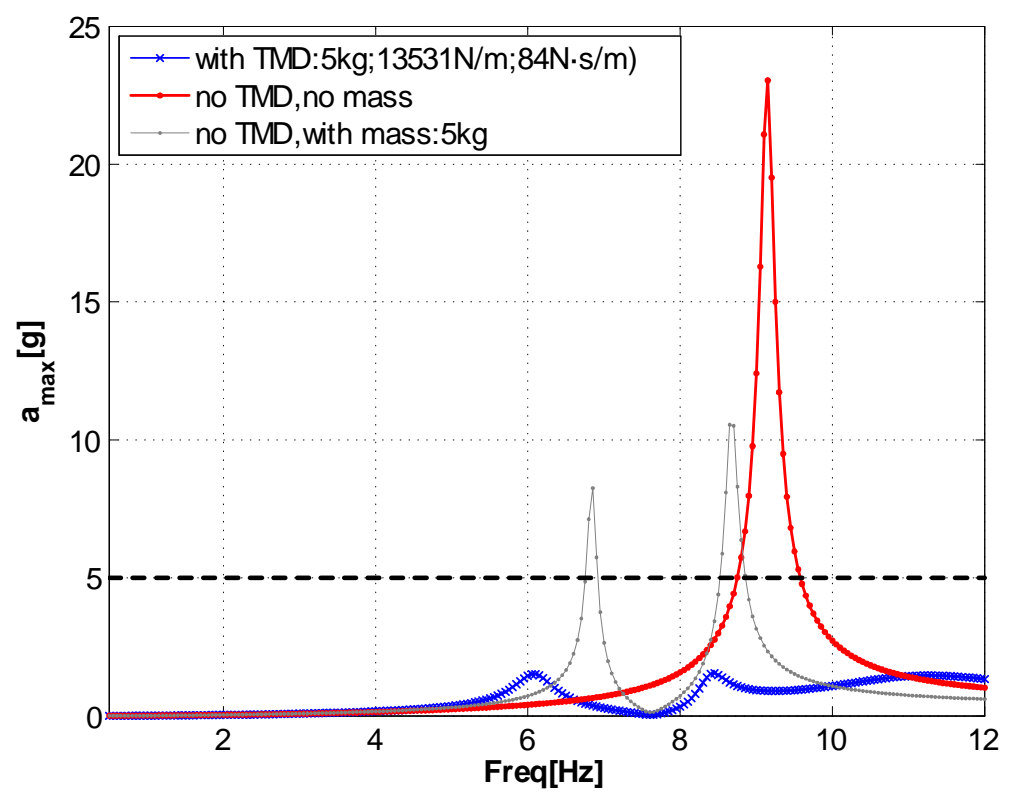

Figura 4-85: Respuesta en frecuencia de aceleraciones en punta de ala izquierda (barrido de frecuencias incrementado)

Como se verá posteriormente, las diferencias de aceleraciones en punta de ala izquierda son pequeñas, menores de un 3\% respecto a los valores que se obtienen con el paso de frecuencia de valor la mitad (caso de 116 frecuencias).

Puede comprobarse en las figuras 4-88, 4-89, 4-90 y 4-91 que la reducida variación de los valores obtenidos de aceleración respecto a los de referencia producen que las parametrizaciones de masa, rigidez y amortiguamiento tengan perfiles muy similares a los de referencia, así como que las gráficas de líneas isoparamétricas se asemejen entre sí. 


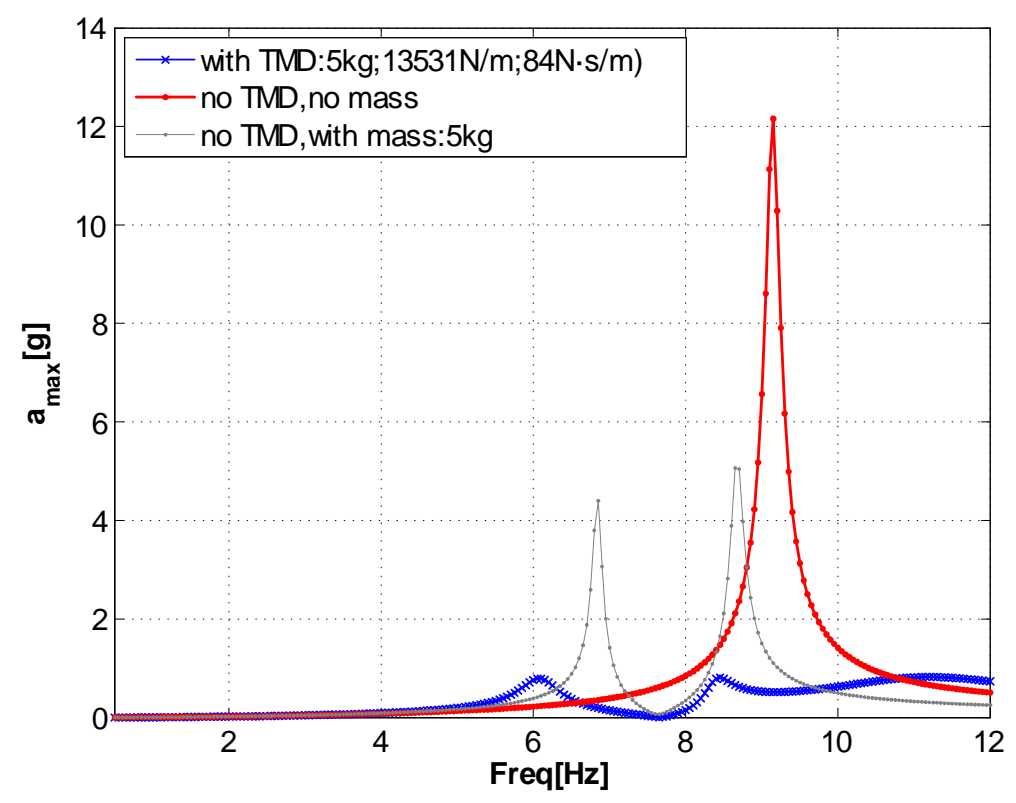

Figura 4-86: Respuesta en frecuencia de aceleraciones en inicio de estrechamiento de semiala izquierda (barrido de frecuencias incrementado)

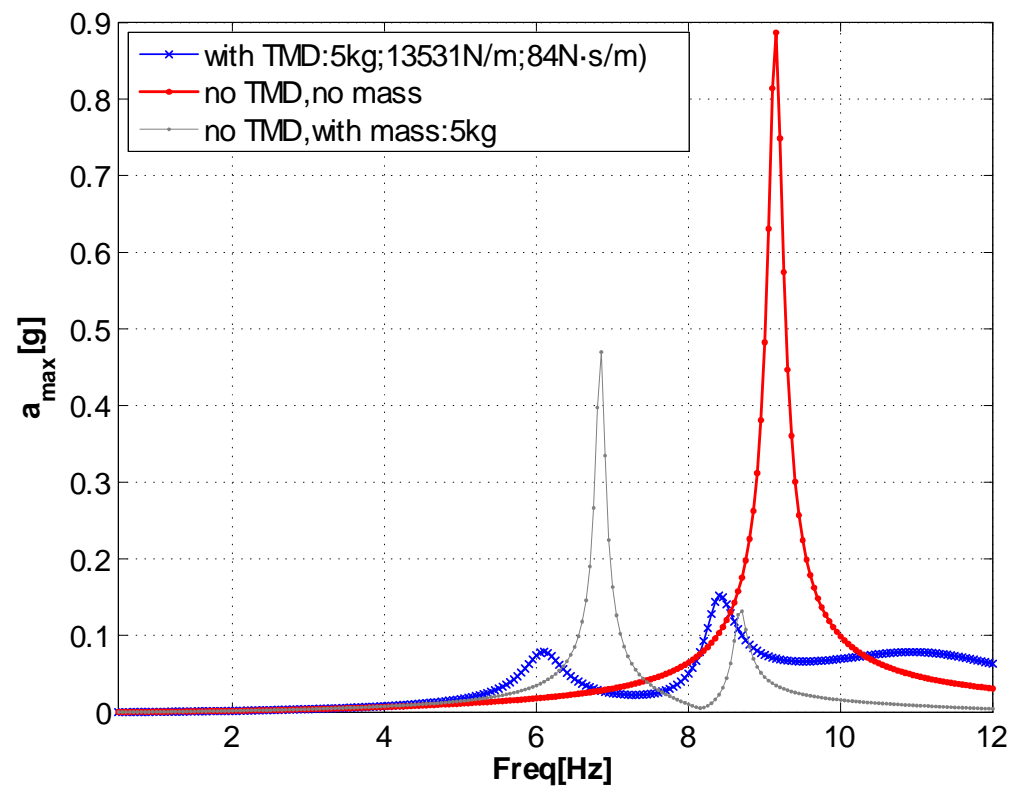

Figura 4-87: Respuesta en frecuencia de aceleración en encastre de semiala izquierda (barrido de frecuencias incrementado) 


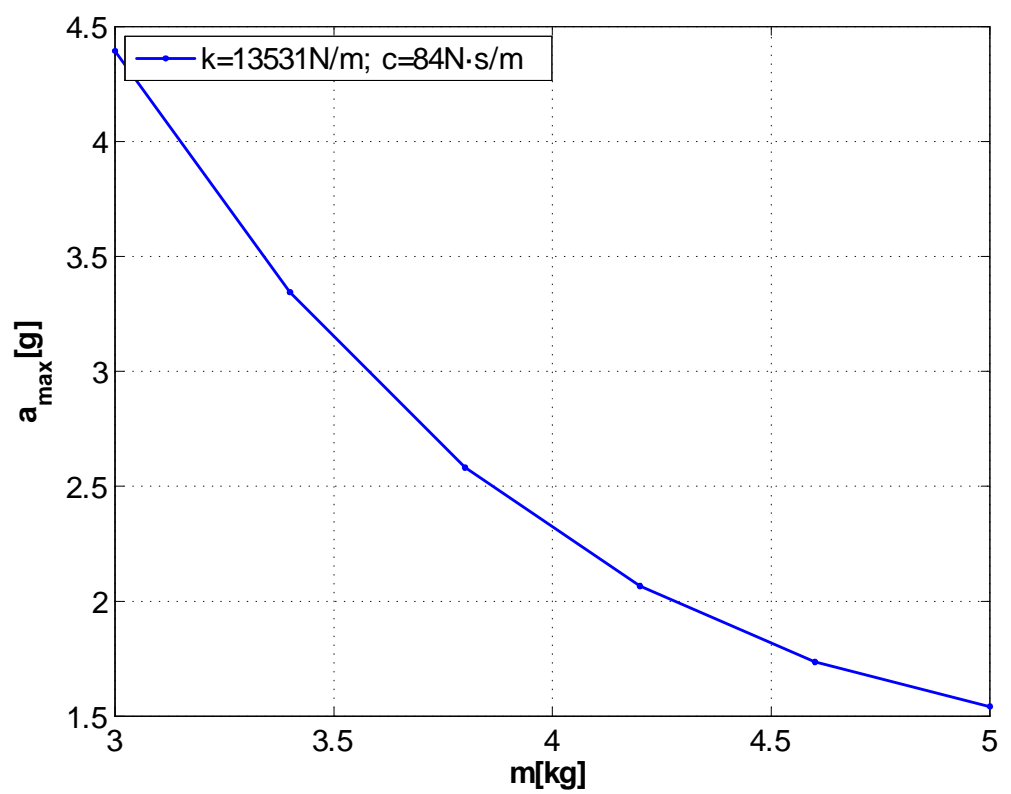

Figura 4-88: Análisis paramétrico en masa (barrido de frecuencias incrementado)

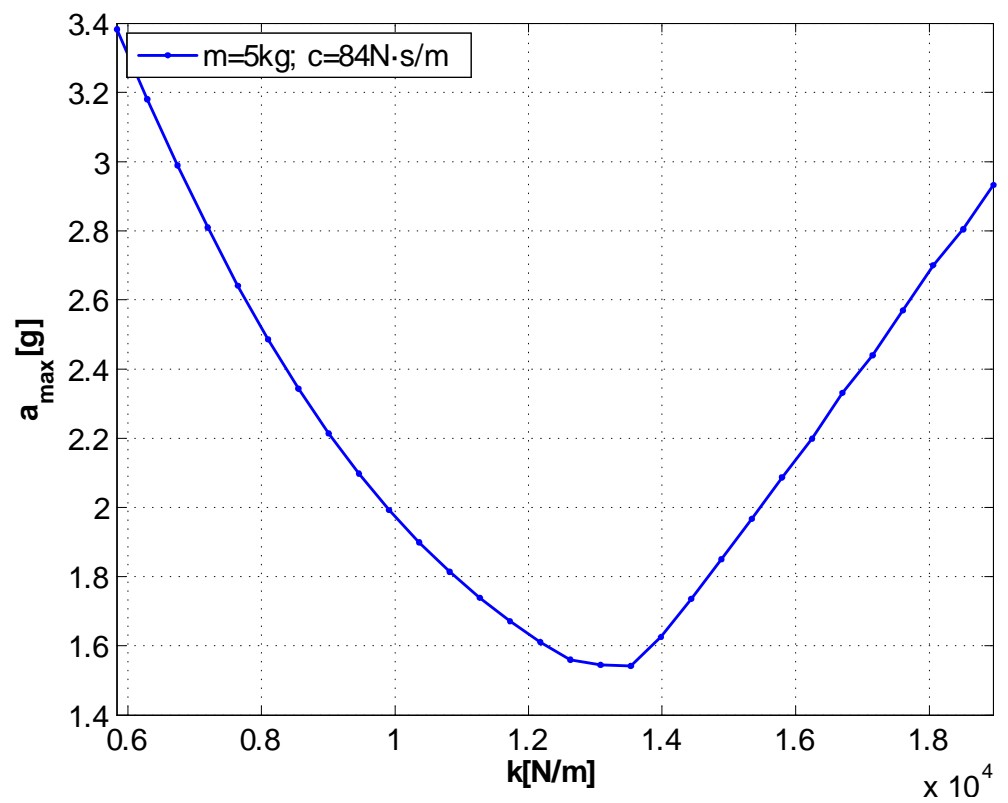

Figura 4-89: Análisis paramétrico en rigidez (barrido de frecuencias incrementado) 


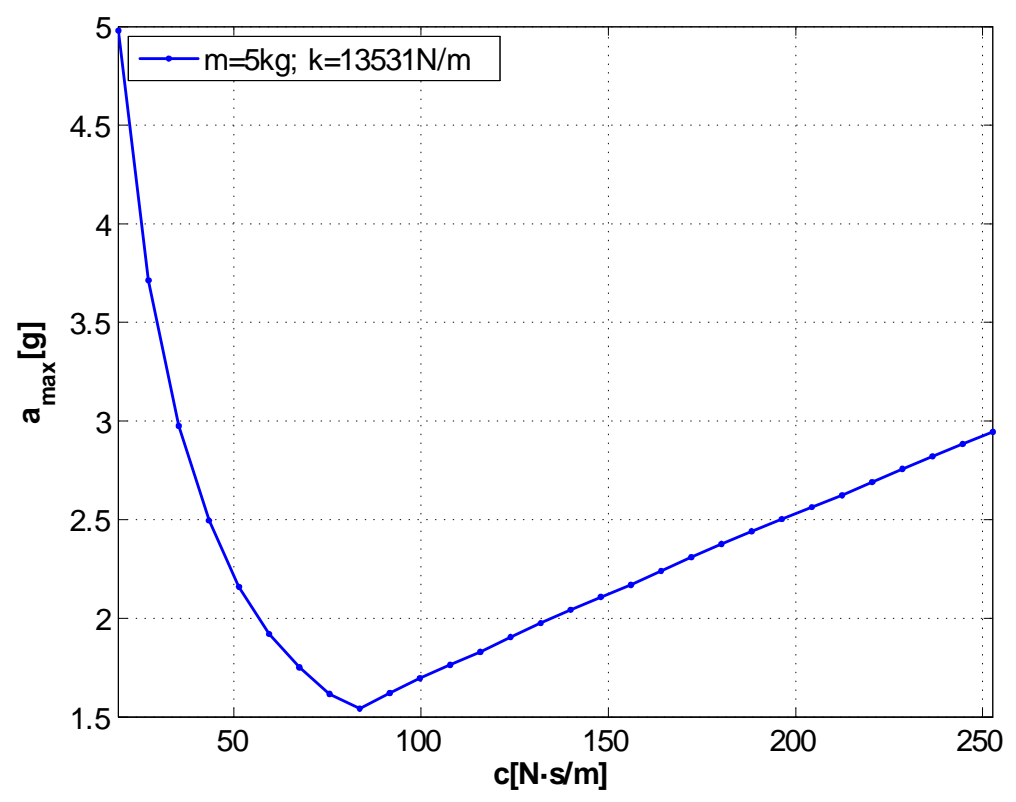

Figura 4-90: Análisis paramétrico en amortiguamiento (barrido de frecuencias incrementado)

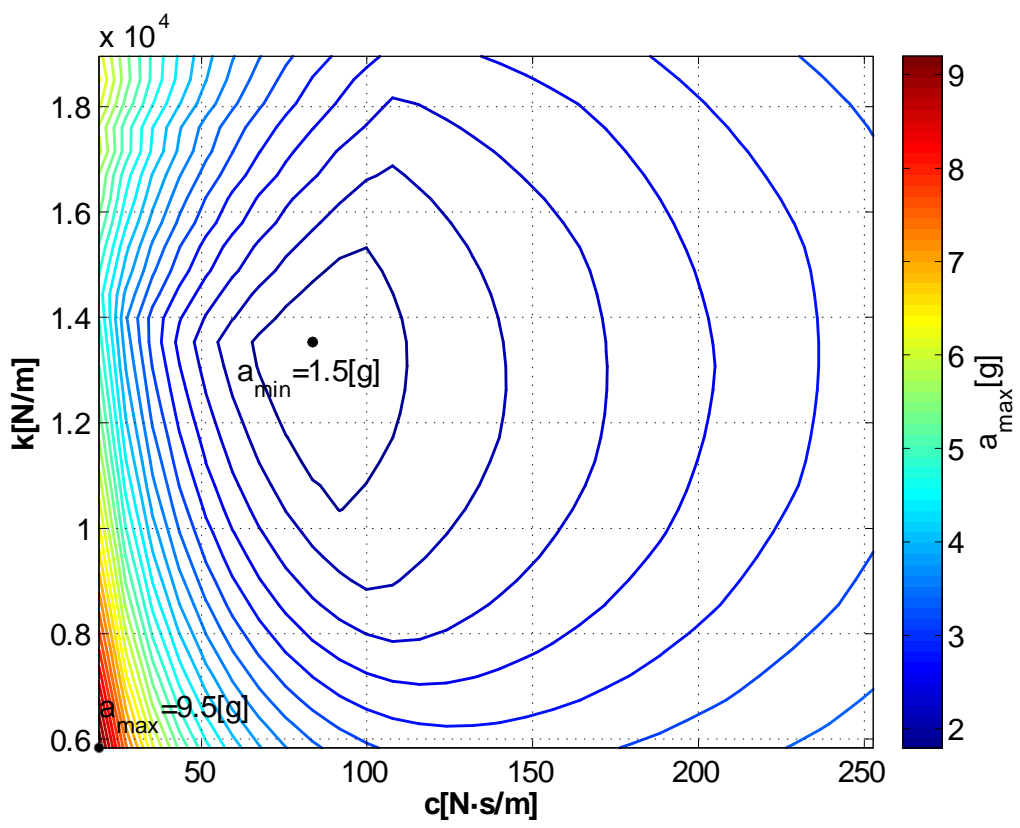

Figura 4-91: Gráfica de líneas isoparamétricas k- c (barrido de frecuencias incrementado) 
Los resultados comparativos con el método empleando 55, 116 y 231 frecuencias se muestran en la siguentes tablas, donde además se comparan con los resultados obtenidos en los análisis ejecutados mediante un programa comercial de elementos finitos. En la tabla 4.14 se comparan los resultados de la respuesta en frecuencia en punta de ala izquierda de aceleración vertical con el equipo añadido pero sin dispositivo de atenuación de la respuesta.

\begin{tabular}{|c|c|c|c|}
\hline Frecuencia $(\mathrm{Hz})$ & $6.8-6.9$ & 7.6-7.7 & 8.7 \\
\hline A-Aceleración con método tesis con 55 frecuencias $\left(\mathrm{m} / \mathrm{s}^{2}\right)$ & 56.3 & 1.9 & 103.1 \\
\hline B-Aceleración con método tesis con 116 frecuencias $\left(\mathrm{m} / \mathrm{s}^{2}\right)$ & 69.9 & 1.3 & 103.1 \\
\hline C-Aceleración con método tesis con 231 frecuencias $\left(\mathrm{m} / \mathrm{s}^{2}\right)$ & 69.9 & 1.3 & 103.5 \\
\hline D-Aceleración con programa comercial $\left(\mathrm{m} / \mathrm{s}^{2}\right)$ & 64.3 & 1.2 & 100.4 \\
\hline Diferencia A-D & $12 \%$ & $51 \%$ & $3 \%$ \\
\hline Diferencia B-D & $9 \%$ & $1 \%$ & $3 \%$ \\
\hline Diferencia C-D & $9 \%$ & $1 \%$ & $3 \%$ \\
\hline
\end{tabular}

Tabla 4.14: Comparación de máximos de aceleraciones con variación barrido frecuencias sin TMD

En la tabla 4.14 se identifica una variación mayor en el primer máximo que en el segundo. Con el primer barrido en frecuencias se localiza un máximo de $56.3 \mathrm{~m} / \mathrm{s}^{2}$ a $6.9 \mathrm{~Hz}$. Cuando se reduce el paso de la frecuencia el máximo se traslada a $6.8 \mathrm{~Hz}$ y a un valor $69.9 \mathrm{~m} / \mathrm{s}^{2}$. De forma análoga, el mínimo calculado con un barrido de 55 frecuencias se corresponde con una frecuencia de $7.7 \mathrm{~Hz}$. Se puede comprobar que las variaciones respecto a los resultados del programa comercial son mayores en el caso del barrido menos denso.

En la tabla 4.15 se comparan los resultados de la respuesta en frecuencia en punta de ala izquierda de aceleración vertical con el equipo añadido incluido el TMD. En este caso, las variaciones respecto a las aceleraciones obtenidas con el modelo FEM son menores para el máximo a $6.8 \mathrm{~Hz}$ pero no para los otros dos extremos del intervalo.

En la última tabla 4.16 se comparan los resultados de la respuesta en frecuencia en punta de ala izquierda de aceleración vertical con los resultados obtenidos en los análisis transitorios ejecutados sobre el modelo completo con el código de análisis comercial. 


\begin{tabular}{|c|c|c|c|}
\hline Frecuencia $(\mathrm{Hz})$ & 6.8 & 7.6 & 8.7 \\
\hline A-Aceleración con método tesis con 55 frecuencias $\left(\mathrm{m} / \mathrm{s}^{2}\right)$ & 13.6 & 0.6 & 14.6 \\
\hline B-Aceleración con método tesis con 116 frecuencias $\left(\mathrm{m} / \mathrm{s}^{2}\right)$ & 14.5 & 0.4 & 14.7 \\
\hline C-Aceleración con método tesis con 231 frecuencias $\left(\mathrm{m} / \mathrm{s}^{2}\right)$ & 14.8 & 0.4 & 15.1 \\
\hline D-Aceleración con programa comercial $\left(\mathrm{m} / \mathrm{s}^{2}\right)$ & 13.2 & 0.4 & 15.1 \\
\hline Diferencia A-D & $3 \%$ & $62 \%$ & $3 \%$ \\
\hline Diferencia B-D & $10 \%$ & $3 \%$ & $2 \%$ \\
\hline Diferencia C-D & $12 \%$ & $0 \%$ & $0 \%$ \\
\hline
\end{tabular}

Tabla 4.15: Comparación de máximos de aceleraciones con variación barrido frecuencias con TMD

\begin{tabular}{|c|c|c|c|}
\hline Frecuencia $(\mathrm{Hz})$ & 6.8 & 7.6 & 8.7 \\
\hline A-Aceleración con método tesis con 55 frecuencias $\left(\mathrm{m} / \mathrm{s}^{2}\right)$ & 13.6 & 0.6 & 14.6 \\
\hline B-Aceleración con método tesis con 116 frecuencias $\left(\mathrm{m} / \mathrm{s}^{2}\right)$ & 14.5 & 0.4 & 14.7 \\
\hline C-Aceleración con método tesis con 231 frecuencias $\left(\mathrm{m} / \mathrm{s}^{2}\right)$ & 14.8 & 0.4 & 15.1 \\
\hline D-Aceleración con programa comercial $\left(\mathrm{m} / \mathrm{s}^{2}\right)$ & 12.8 & 0.4 & 14.3 \\
\hline Diferencia A-D & $6 \%$ & $78 \%$ & $2 \%$ \\
\hline Diferencia B-D & $13 \%$ & $7 \%$ & $3 \%$ \\
\hline Diferencia C-D & $16 \%$ & $10 \%$ & $6 \%$ \\
\hline
\end{tabular}

Tabla 4.16: Comparación de máximos de aceleraciones con análisis transitorio con variación barrido frecuencias

Puede verse que en la mayoría de los casos las diferencias entre los resultados obtenidos con los barridos de 116 y 231 frecuencias son similares y se mantienen por debajo del $16 \%$ para los máximos. El resultado de comparación del mínimo para el caso de 55 frecuencias es superior. Hay que recordar que para el caso calculado con el paso de frecuencia reducido tanto la frecuencia a la que se producen los extremos como la combinación óptima de propiedades del TMD varían respecto a los otros dos casos. 


\section{Capítulo 5}

\section{CONCLUSIONES}

\subsection{Discusión sobre los resultados}

Se ha desarrollado un método que permite realizar análisis dinámicos de modificaciones locales con gran eficiencia computacional gracias a la reducción de los tamaños de las matrices involucradas al basarse en el subespacio modal de un grupo reducido de grados de libertad. Se ha implementado un código en MatLAB que, tras extraer los autovalores de los modos considerados del modelo de elementos finitos y los datos correspondientes a los grados de libertad de interés, construye las matrices de masa, rigidez y amortiguamiento modificadas y resuelve las ecuaciones del movimiento en el campo modal complejo. Los reducidos tiempos de ejecución de los análisis permiten que este procedimiento sea usado en análisis paramétricos de grandes estructuras.

Para mostrar su validez, se ha realizado un análisis paramétrico para seleccionar los parámetros de masa, rigidez y amortiguamiento de un sistema compuesto por un equipo y un elemento amortiguador.

En resumen, se considera un caso de aplicación a una estructura real, con un posible caso de carga y con el que se pretende validar diversos aspectos del método de aceleración de análisis dinámicos:

- Reducción del modelo por truncatura modal

- Reducción del modelo mediante la selección de grados de libertad significativos del modelo 
- Implementación de la adición de nuevos grados de libertad mediante la adición de un nuevo nodo para la simulación del equipo.

- Modificación local de la masa de la estructura mediante la adición de nuevas propiedades de masa

- Modificación local de la rigidez de la estructura mediante la adición de nuevas propiedades de rigidez

- Modificación local del amortiguamiento de la estructura mediante la adición de nuevas propiedades de amortiguamiento

- Realización de diversos análisis paramétricos para la verificación de los requisitos considerados

- Se han ejecutado numerosos análisis para lograr identificar la mejor combinación de dichos parámetros. Se ha validado que el conjunto de parámetros seleccionado cumple con los requisitos.

- También se ha comparado el procedimiento con los correspondientes análisis dinámicos realizados sobre el modelo de elementos finitos mediante un código comercial, tanto la respuesta en frecuencia como la respuesta temporal en un análisis transitorio.

En principio, según el desarrollo teórico expuesto, los errores de aproximación del método en estudio provienen de los debidos a la truncatura modal y a la extracción de los autovalores. En cambio, puede haber múltiples causas de las diferencias entre los resultados obtenidos empleando el procedimiento propuesto y los resultados provenientes del código comercial.

Una de ellas puede ser el método de cálculo de los autovalores, ya que los códigos empleados en cada caso, MatLAB y MSC.Nastran, pueden no usar los mismos métodos. Esto es aplicable no solo al problema de autovalores reales sino también al complejo. Por otro lado, al realizar los análisis paramétricos en los códigos generados con MatLAB solo se emplea una extracción de autovalores inicial procedente de Nastran. En cambio, si se hiciesen todos los análisis equivalentes en Nastran, se recalcularían los modos en cada análisis con el coste computacional que ello conllevaría. Esto es otro factor que agiliza el método de aceleración, de forma que se extrae 
inicialmente la información necesaria del modelo de elementos finitos una única vez, permitiendo gran autonomía y flexibilidad.

En segundo lugar hay que tener en cuenta que se emplean distintas maneras para la inclusión del amortiguamiento de la estructura del ala. En el procedimiento propuesto, se introduce como un amortiguamiento modal, una vez se han extraido los autovalores reales con los que se realiza la truncatura modal para la estructura sin el equipo. En cambio, en el código comercial se simula el amortiguamiento modal mediante un amortiguamiento estructural equivalente sobre los modos reales con la masa y rigidez que aporta el equipo. Esta es la causa principal de diferencias entre los resultados de los dos métodos tal y como se valida gracias a la comparación para el caso sin amortiguamiento. Una posible diferencia puede ser que los modos despreciados tienen una componente estática no incluida. Otra posible diferencia puede deberse a que esos modos tienen un amortiguamiento muy alto se se usa la aproximación de Rayleigh.

En la comparación con el análisis temporal mediante el esquema de integración implícito Newmark- $\beta$ hay dos factores que pueden resultar determinantes. En primer lugar, este análisis emplea el modelo completo de elementos finitos de la estructura a diferencia de los que emplean un número reducido de modos propios. Como se ha comentado, este, al igual que en la mayoría de métodos de reducción de modelos para análisis dinámicos, es una de las fuentes de pérdida de precisión por lo que evaluar este aspecto puede ser de importancia. En este trabajo se han realizado análisis con 21, 60, 100 y 151 modos aunque sería interesante ampliar el estudio de este factor y la posible mejora de la precisión empleando, por ejemplo, alguno de los métodos de optimización de los resultados gracias al residuo modal estático.

La segunda componente esencial a tener en cuenta en la comparación de los resultados con el análisis implícito con esquema Newmark- $\beta$ es el número de ciclos de estabilización de la respuesta temporal. Hay que tener en cuenta que, aunque el método estudiado permite el cálculo de respuestas transitorias, en el caso de aplicación se ha obtenido la respuesta permanente por lo que debe compararse los resultados sin tener en cuenta las variaciones de los primeros ciclos previos a la estabilización de la solución. Esta estabilización depende de factores como el amortiguamiento de la estructura.

Hay otros factores secundarios que también pueden condicionar la respuesta transitoria calculada y que intervienen en las diferencias entre la técnica propuesta y los resultados estándar 
del programa comercial como el método de introducción de la carga dependiente del paso de integración o la forma de introducción del amortiguamiento modal en el cálculo con el esquema Newmark- $\beta$.

Teniendo lo anterior en cuenta, se puede analizar cada uno de los resultados de los tipos de análisis estudiados.

Los análisis paramétricos con amortiguamiento modal de un $1 \%$ sirven como base comparativa para el resto de análisis. Además de servir de caso de aplicación frente a unos requisitos para la selección de los parámetros adecuados, se han comparado sus resultados con los obtenidos con el código comercial.

Las diferencias respecto a este último son menores de un 10\% en los máximos y mínimos que se encuentran dentro del intervalo de frecuencias estudiado tanto en el caso de adición de equipo como en el caso de adición de equipo con sistema de atenuación incorporado. La comparación con la estructura completa mediante el análisis transitorio permanece en valores muy similares (8\% y 16\%) excepto para el valor mínimo (57\%) cuya diferencia porcentual se estima que se debe a la influencia de la pequeña magnitud de los valores $\left(0.4 \mathrm{~m} / \mathrm{s}^{2}\right.$ frente a $\left.0.9 \mathrm{~m} / \mathrm{s}^{2}\right)$. Las diferencias del máximo del entorno de $8 \mathrm{~Hz}$ son menores que las del primer máximo a $6 \mathrm{~Hz}$ aproximadamente.

Cuando se incrementa el amortiguamiento modal de la estructura los resultados muestran más diferencias respecto a los valores obtenidos con el código comercial. Con un $5 \%$ de amortiguamiento modal las diferencias de los resultados de adición de equipo en la punta de ala se disparan hasta aproximadamente el $26 \%$, mientras que si se considera el equipo con amortiguador las diferencias se mantienen por debajo de un $29 \%$. La disparidad frente al análisis de respuesta temporal se mantiene por debajo del $20 \%$.

Dadas las diferencias al variar el amortiguamiento modal del $1 \%$ al $5 \%$ se han ejecutado unos análisis con amortiguamiento nulo para intentar detectar los efectos debidos a este factor. En este caso, la comparación realizada arroja una diferencia máxima de un $3 \%$ que puede considerarse despreciable. Esto valida el argumento expuesto anteriormente por el que se justificaban las diferencias entre los resultados del método de aceleración propuesto en la tesis y los resultados obtenidos con el programa comercial de elementos finitos basándose en la distinta forma de introducir el amortiguamiento. Esto implica que el método de aceleración estudiado 
ofrece semejantes resultados a los del código comercial por lo que se puede concluir que es igual de válido que él.

En cuanto a la comparación del caso con amortiguamiento nulo con el análisis transitorio de la estructura completa, las diferencias son menores de un 31\% salvo para el mínimo localizado a $7.6 \mathrm{~Hz}$ que sube hasta el $97 \%$ probablemente por los reducidos valores a comparar.

Con el fin de estudiar si las diferencias entre resultados podían deberse en gran parte a la truncatura modal realizada se ha comparado los análisis en los que se han retenido 21, 60, 100 y 151 modos. Las diferencias de los resultados obtenidos respecto a los análisis transitorios del modelo completo ofrecen diferencias máximas de un 16\%, siendo de un $13 \%$ para el mejor caso que es el correspondiente a 151 modos. Los resultados de los análisis ejecutados empleando el método de aceleración con diferente número de modos considerados son muy similares, con variaciones máximas de un $3 \%$ de unos respecto a otros.

Hay que recordar que dado que las comparaciones se realizan entre los valores máximos es importante su forma de identificación. De esta manera, factores como el paso de frecuencia con el que se recorre el intervalo de estudio puede producir que valores precisos de extremos agudos, de gran amplitud en un ancho de banda pequeño, sean difíciles de determinar adecuadamente. Este efecto puede combinarse con el desplazamiento de los extremos en la frecuencia haciendo que la comparación no sea sencilla. Cuando las condiciones del problema cambian pueden variar las relaciones entre los parámetros del problema, de forma que el conjunto óptimo de parámetros no sea el mismo y, por lo tanto, su curva también se vea modificada.

Uno de estos condicionantes se ha estudiado variando al doble y a la mitad el incremento de la frecuencia pasando de $0.1 \mathrm{~Hz}$ en los análisis de referencia a 0.2 y $0.05 \mathrm{~Hz}$ respectivamente entre $0.5 \mathrm{~Hz}$ y $12 \mathrm{~Hz}$. La idea es observar si este cambio produce una mejor detección de los extremos, como se ha dicho, tanto por la identificación más precisa de su valor como de la frecuencia a la que ocurren. De hecho, la curva óptima de atenuación de la señal cambia de parámetros pasando de una rigidez de $13313 \mathrm{~N} / \mathrm{m}$ y un amortiguamiento de $81 \mathrm{Ns} / \mathrm{m}$ a una rigidez de 13513 N/m y un amortiguamiento de 84 Ns/m. En la mayoría de los extremos sucede así en los casos de análisis de respuesta en frecuencia pero no cuando se comparan los resultados con los análisis transitorios de la estructura completa.

Para estudiar la eficiencia temporal del método se puede ver la tabla 5.1 en donde se reúnen 
los tiempos de cálculo comparables de análisis de respuesta en frecuencia para distintos números de modos propios considerados y paso de frecuencia y sus equivalentes ejecutados con un código comercial de elementos finitos. Puede comprobarse que el aumento de modos considerados o frecuencias incrementa el tiempo de cálculo.

\begin{tabular}{|c|c|c|c|}
\hline $\mathbf{N}^{o}$ modos & Paso frec. (Hz) & Tiempo cód. comercial (s) & Tiempo método (s) \\
\hline 21 & 0.1 & 579 & 0.4 \\
\hline 60 & 0.1 & 864 & 0.4 \\
\hline 100 & 0.1 & 1026 & 0.7 \\
\hline 151 & 0.1 & 1200 & 1.1 \\
\hline 151 & 0.2 & 1157 & 0.9 \\
\hline 151 & 0.05 & 1404 & 1.5 \\
\hline
\end{tabular}

Tabla 5.1: Tiempos de computación de análisis en frecuencia

En cualquiera de los casos, el tiempo empleado en la ejecución con el método es claramente inferior (aproximadamente dos órdenes de magnitud) respecto al mismo tipo de análisis realizado mediante el código comercial. Es importante destacar que para el caso de referencia ejecutado con 151 modos propios retenidos, amortiguamiento modal de un $1 \%$ y un paso de frecuencia de $0.1 \mathrm{~Hz}$, cada análisis de respuesta en frecuencia realizado con un código comercial empleó 1200 segundos mientras que cada análisis utilizando la técnica propuesta se ejecuta en 1.1 segundos, mostrándose por lo tanto una gran reducción de tiempo de computación (ver tabla 5.2). La implementación del método es sencilla y permite mantener un gran control y flexibilidad sobre todos los aspectos del análisis paramétrico. Esto permite proponer complejas parametrizaciones con un elevado número de parámetros involucrados. La ganancia en tiempo respecto al cálculo con la estructura completa es aún mayor si se tiene en cuenta que los análisis de integración numérica del modelo completo para intervalos de tiempos de 2.4 s emplean unos 4500 segundos en ofrecer resultados.

Si se emplean los anteriores tiempos por análisis como tiempos medios, se puede estimar que el tiempo necesario para ejecutar los cerca de 115000 análisis transitorios de los que se han 


\begin{tabular}{|c|c|c|}
\hline Análisis caso de referencia & Tiempo por análisis (s) & Tiempo total (h) \\
\hline Modelo completo con código comercial & 4500 & 96000 \\
\hline Modelo reducido con código comercial & 1200 & 25600 \\
\hline Modelo reducido con método propuesto & 1.1 & 23.4 \\
\hline
\end{tabular}

Tabla 5.2: Tiempos de computación del caso de análisis de referencia

mostrado resultados en esta tesis habrían consumido casi 6000 días (16 años) en vez de las 35 horas que aproximadamente se han empleado gracias al método de aceleración propuesto.

Adicionalmente, como el análisis de sensibilidad considera solo partes reducidas de las matrices de masas, amortiguamiento y rigidez los archivos electrónicos requieren mucho menor tiempo de computación y capacidad de almacenamiento que los análisis de la estructura completa. Los tamaños de los ficheros, KB frente a cientos de MB, además permiten un manejo más sencillo y menos potencia de cálculo en los ordenadores que los administren. 


\subsection{Conclusiones generales}

En este trabajo se ha investigado la aplicación de una técnica de reducción de modelos de elementos finitos para la aceleración de análisis paramétricos de modificaciones estructurales en grandes estructuras bajo cargas dinámicas. Se ha presentado su necesidad y la base teórica que lo sustenta y que permite entender el alcance de sus características.

La eficiencia del método se ha probado en el caso de estudio de un análisis paramétrico de una modificación de un modelo del ala del UAV MILANO consistente en la adición en punta de ala de un equipo dotado con un sistema de limitación de la respuesta dinámica. Tras la ejecución de diversos análisis paramétricos que han permitido cumplir con los requisitos del problema y la selección del conjunto óptimo de parámetros, los resultados obtenidos se han comparado a los ofrecidos por programas de elementos finitos habitualmente usados en la industria aeroespacial, tanto con la respuesta en frecuencia como con la respuesta temporal de un análisis transitorio.

Se han discutido las causas de las diferencias y se ha estudiado la influencia de algunos de los factores como el amortiguamiento modal de la estructura, el número de modos retenidos en la truncatura modal o el valor del incremento de frecuencia en el cálculo de la respuesta.

De acuerdo con los resultados obtenidos, se considera que la aplicación del método de aceleración de análisis dinámicos propuesto ofrece unos resultados adecuados en la precisión de la respuesta lograda ya que se ha verificado que ofrece resultados con diferencias despreciables frente a los obtenidos con el código comercial de elementos finitos para el mismo caso de análisis y diferencias reducidas respecto a análisis transitorios de la estructura completa.

Esto hace que, teniendo en cuenta los considerables ahorros de tiempo, potencia de cálculo y necesidad de almacenamiento de los archivos numéricos, dicho método sea una opción recomendable y particularmente útil para el análisis paramétrico de grandes estructuras con modificaciones locales. 


\subsection{Trabajos futuros}

Al realizar el presente trabajo han surgido posibles líneas de investigación en relación con el ámbito de aplicación del procedimiento propuesto tanto desde el punto de vista de su alcance como de su uso.

Primeramente podrían extenderse los estudios iniciados determinando las posibles relaciones con factores como el número de modos retenidos en la truncatura modal de forma que pueda determinar a priori los límites de la extracción de autovalores de la estructura completa. La precisión de la truncatura podría revisarse con algún tipo de corrección estática del residuo modal. La determinación de algoritmos de optimización de la selección del número de intervalos de frecuencia en el rango de estudio y de selección de extremos podría proporcionar mejoras en la precisión y eficiencia del método ya que en la tesis doctoral las respuestas en frecuencia se dan para valores equiespaciados.

El estudio de la comparación con los análisis transitorios mediante el uso de otros esquemas de cálculo o incluso con la comparación con la solución transitoria obtenida directamente con el método considerado puede resultar interesante.

Por otro lado, puede realizarse un estudio comparativo de diversos métodos de reducción de modelos de elementos finitos de forma que se pueda contrastar la eficiencia de la técnica propuesta e identificar sus límites prácticos de aplicación en relación con otros métodos como por ejemplo la selección de diversos rangos de frecuencias de estudio o la influencia en los resultados de los diversos tipos de amortiguamiento en la estructura no modificada. También puede analizarse el impacto en el uso del método de la implementación de diversas formas de cálculo de los autovalores complejos.

Otro de los posibles desarrollos relacionados con el método de aceleración de análisis paramétricos es su posible implementación en los programas comerciales más conocidos a través de aplicaciones que se incorporen con los lenguajes de programación de que dispone cada programa como por ejemplo DMAP para MSC.NASTRAN. Esto proporcionaría una herramienta muy potente en el mismo entorno del código comercial de análisis de elementos finitos. 


\section{Referencias}

[Acquaroli et al., 2005] Acquaroli, L., Morelli, B., \& Onorati, F. 2005. MITA MinisatellitePayload Damping System. ESMATS Symposium. ESA SP-581.

[Adhikari \& Phani, 2004] Adhikari, S., \& Phani, A. 2004. Rayleigh Classical Damping Revisited.

[ANSYS, 2013] ANSYS. 2013. ANSYS Mechanical User's Guide. Ansys, Inc.

[Armendariz et al. , 2015] Armendariz, I., Olarrea, J., \& Garcia-Martinez, J. 2015. Parametric Analysis of a Highly Dynamical Phenomena Caused by a Propeller Blade Loss. Engineering Failure Analysis, 57, 528-543.

[Aryana \& Bahai, 2003] Aryana, F., \& Bahai, H. 2003. Sensitivity analysis and modification of structural dynamic characteristics using second order approximation. Engineering Structures, 25(1279-1287).

[Avitable, 2003] Avitable, P. 2003. Twenty Years of Structural Dynamic Modification - A Review. Sound and Vibration.

[Bahai et al. , 2002] Bahai, H., Farahani, K., \& Djoudi, M-S. 2002. Eigenvalue inverse formulation for optimising vibratory behaviour of truss and continuous structures. Computers and Structures, 2397-2403.

[Baier \& Reindl, 2003] Baier, H., \& Reindl, M. 2003. Adaptative Structures and Mechatronic Components for Vibration and Shape Control of Satellite Precision Payloads. ESMATS Symposium. ESA SP-524. 
[Bathe, 1996] Bathe, K.J. 1996. Finite Element Procedures. Prentice Hall.

[Benner et al. , 2009] Benner, P., Bonin, T., Fasvender, H., Saak, J., Soppa, A., \& Zaek, M. 2009. Novel Model Reduction Techniques for Control and Machine Tools. ANSYS conference and 27th CADFEM users meeting.

[Besselink, 2013] Besselink, B. 2013. A comparison of model reduction techniques from structural dynamics, numerical mathematics and systems and control. Journal of Sound and Vibration, 332, 4403-4422.

[Brotons et al. , 1999] Brotons, P., Cabrera, E., García-Paredes, S., Gómez-Molinero, V., Luengo, P., Pascual, J., \& Uribarri, J. 1999. ENVISAT Shock Attenuation Device Developement. European Conference on Spacecraft Structures, Materials and Mechanical Testing. ESA SP-468.

[Camarasa et al. , 2005] Camarasa, P., Demerville, T., Plagne, A., \& Kiryenko, S. 2005. Shock Attenuation System for Spacecraft and Adaptor. European Conference on Spacecraft Structures, Materials and Mechanical Testing.

[Carpi et al. , 2005] Carpi, F., Sommer-Larsen, P., Rossi, D., Gaudenzi, P., Lampani, L., Campanile, F., Pfeiffer, E., Neri, G., \& Baldacci, S. 2005. Electroactive Polymers: New Materials for Spacecraft Structures. ESMATS. ESA SP-581.

[Chen et al. , 2011] Chen, X., Wang, H., \& Ding, Y. 2011. Multi-Domain Parallel Computing for Strength Analysis of Whole Aircraft Model. Journal of Software Engineering and Applications, 546-549.

[Choi \& Wereley, 2004] Choi, Y., \& Wereley, N. 2004. Shock Isolation Systems Using Magnetorheological Dampers. Smart Structures and Materials. Vol. 5386.

[Chopra, 1995] Chopra, A. K. 1995. Dynamic os Structures. Prentice Hall.

[Craig \& Bampton, 1968] Craig, R., \& Bampton, M. 1968. Coupling of Substructures for Dynamic Analyses. AIAA Journal, 6(7), 1313-1319.

[Cuschieri, 1990] Cuschieri, J. 1990. Parametric and Experimental Analysis Using a Power Flow Approach. Tech. rept. NASA Contractor Report 181990. 
[Dassault, 2007] Dassault. 2007. Abaqus Analysis User's Manual. Dassault Systemes.

[Doengi et al. , 1998] Doengi, F., Burnage, S., Cottard, H., \& Roumeas, R. 1998. Lander Shock-Alleviation Techniques. ESA Bulletin 93.

[EASA, 2007] EASA. 2007. Certification Specifications for Large Aeroplanes CS-25. Amendment 3 edn. European Aviation Safety Agency.

[Farstad \& Singh, 1996] Farstad, J., \& Singh, R. 1996. Effects of Modal Truncation Errors on Transmitted Dynamics Power Estimates in Discretely Joint Component Assemblies. Journal Acoustic Society of America, 100(5), 3144-3158.

[Fung, 1993] Fung, Y. 1993. An introduction to the Theory of Aeroelasticity. Dover Publications, Inc.

[Garatani, 1999] Garatani, K. 1999. Feasibility Study of GeoFEM for Solving 100 Millions DOF Problems. 1st ACES Workshop Proceedings.

[Gavin, 2014] Gavin, H. 2014. Classical Damping, Non-Classical Damping and Complex Modes. CEE 541. Structural Dynamics.

[Guyan, 1965] Guyan, R. J. 1965. Reduction of Stiffness and Mass Matrices. AIAA Journal, $3(2), 380$.

[Hanich et al. , 2001] Hanich, I., Preaumont, A., \& Loix, N. 2001. Piezoelectric Stewart Platform for General Purpose Active Damping Interface and Precision Control. ESMATS Symposium. ESA SP-480.

[Harris, 1961] Harris, C. 1961. Shock and Vibration Hanbook. McGraw-Hill.

[Hermans et al. , 2000] Hermans, L., Mas, P., Leurs, W., \& Boucart, N. 2000. Estimation and Use of Residual Modes in Modal Coupling Calculations: A Case Study. IMAC XVIII.

[Hoen, 2005] Hoen, C. 2005. An Engineering Interpretation of the Complex Eigensolution of Linear Dynamics Systems. IMAC XXIII.

[Hurty, 1965] Hurty, W. C. 1965. Dynamic Analysis of Structural Systems Using Component Modes. AIAA Journal, 3(4), 678-685. 
[Johson \& Wilke, 2000] Johson, C., \& Wilke, P. 2000. Whole-Spacecraft Shock Isolation System. SPIE 9th Annual International Symposium on Smart Structures and Materials, Damping and Isolation.

[Laan \& Tooren, 2004] Laan, A., \& Tooren, M. 2004. Parametric Modeling of Movables for Structural Analysis. AIAA/ASME/ASCE/AHS/ASC Structures, Structural Dynamics and Material Conference.

[Lázaro \& Pérez-Aparicio, 2013] Lázaro, M., \& Pérez-Aparicio, J. L. 2013. Multiparametric computation of eigenvalues for linear viscoelastic structures. Computers and Structures, $67-$ 81.

[Lefevre et al. , 2003] Lefevre, J., Gabbert, U., \& Koppe, H. 2003. Simulation of Piezoelectric Controlled Smart Structures by the Finite Element Method Including Vibroacoustic Effects. International Congress on sound and vibration.

[Leontiev, n.d.] Leontiev, M. Truncation Errors in the Modal Vibration Analysis of Rotor Systems.

[Li \& Gunter, 1981] Li, D., \& Gunter, E. 1981. A Study of the Modal Truncation Error in the Component Mode Analysis of a Dual-Rotor System. Journal of engineering for power.

[Li \& He, 1999] Li, T., \& He, J. 1999. Local Structural Modification using mass and stiffness changes. Engineering Structures, 21, 1028-1037.

[Liu et al. , 2000] Liu, D., Chung, H., \& Chang, W. 2000. The Errors Caused by Modal Truncation in Structure Dynamic Analysis. IMAC XVIII.

[Lusebrink \& Brinkspalink, 1994] Lusebrink, H., \& Brinkspalink, J. 1994. Aircraft Loads Due to Turbulence and their Impact on Design and Certification. Tech. rept. Advisory Group for Aerospace Research and Development.

[Majkut, 2010] Majkut, L. 2010. Eigenvalue based inverse model of beam for structural modification and diagnosis. Part I: Theoretical formulation. Latin American Journal of Solids and Structures, 423-436. 
[Martinez-Agirre \& Elejabarrieta, 2011] Martinez-Agirre, M., \& Elejabarrieta, M. J. 2011. Higher order eigensensitivities-based numerical method for the harmonic analysis of viscoelastically damped structures. International Journal for Numerical Methods in Engineering, 1280-1296.

[Masuda et al. , 2005] Masuda, A., Sone, A., Yamamura, T., Ni, Q., \& Zhang, R. 2005. Shock Absortion Capability of Woven SMA Skin. Smart Structures and Materials. SPIE Vol. 5757.

[Mathworks, 2013] Mathworks. 2013. MatLAB Manual. Mathworks.

[M.Lázaro et al. , 2012] M.Lázaro, Pérez-Aparicio, J.L., \& Epstein, M. 2012. Computation of eigenvalues in proportionally damped viscoelastic structures based on the fixed-point iteration. Applied Mathematics and Computation, 3511-3529.

[Mobley, 1999] Mobley, R. K. 1999. Vibration Fundamentals. Newnes.

[Montáns \& Muñoz, 2013] Montáns, F. J., \& Muñoz, I. 2013. Dynamic Analysis of Structures for the Finite Element Method. UNED.

[Mortzfel \& Ma, 2011] Mortzfel, M., \& Ma, F. 2011. The Decoupling of Linear Dynamical Systems. III ECOMAS thematic conference on Computational Methods in Structural Dymanics and Earthquake Engineering.

[Mourelatos et al. , 2012] Mourelatos, Z. P., Angelis, D., \& Skarakis, J. 2012. Advances in Vibration Engineering and Structural Dynamics. InTech.

[Mourllion \& Birouche, 2013] Mourllion, B., \& Birouche, A. 2013. Modal Truncation for Linear Hamiltonian Systems: A Physical Energy Approach. Taylor and Francis. Chap. 28, pages $187-202$.

[MSC.Software, 2004] MSC.Software. 2004. MSC.Nastran Version 70. Advanced Dynamics Analysis Users's Guide. MSC.Software Corporation.

[MSC.Software, 2012] MSC.Software. 2012. MSC.Nastran Superelements Users's Guide. MSC.Software Corporation.

[MSC.Sofware, 2012] MSC.Sofware. 2012. MSC Nastran 2012 Dynamic Analysis Userst's Guide. 
[Nabag et al. , 2010] Nabag, M., AlRadhawi, M., \& Bettayeb, M. 2010. Model Reduction of Flat-Plate Solar Collector Using Time-Space Discretization. IEEE.

[Nad, 2007] Nad, M. 2007. Structural dynamic modification of vibrating systems. Applied Mathematics and Computation, 1, 203-214.

[O'Callahan, 2003] O'Callahan, J. 2003. System Matrix Optimization Using a Complex Analitical Model Improvement (CAMI). SEM.

[P. Wallack, 1988] P. Wallack, P. Skoog, M. Richardson. 1988. Simultaneous Structural Dynamics Modification (S2DM). IMAC, VI.

[Papai et al. , 2012] Papai, F., Adhikari, S., \& Wang, B. 2012. Estimation of Modal Damping on Unmeasured Modes. Slovak Journal of Civil Engineering, XX(4), 17-27.

[Pereira, 2001] Pereira, C. 2001. Mechanisms of the ROSETTA High Gain Antenna. 9th ESMATS symposium, ESA SP-480.

[Pratt \& Walker, 1954] Pratt, K. G., \& Walker, W. G. 1954. A Revised Gust-Load Fornula and a Re-Evaluation of V-G Data Taken on Civil Transport Airplanes from 1933 to 1950. Tech. rept. NACA.

[Qu, 2004] Qu, Z. 2004. Model Order Reduction Techniques.

[R. J. Allemang, 1987] R. J. Allemang, D. L. Brown. 1987. Experimental Modal Analysis and Dynamic Component Synthesis. Tech. rept. AFWAL.

[Rayleigh, 1945] Rayleigh, J. W. S. 1945. The Theory of Sound. Dover Publications.

[Rey, 2013] Rey, J. 2013. Aplicación de Algoritmos Paramétricos de Generación Formal En el Diseño Estructural. La Ópera de Sidney Desde Una Perspectiva Contemporánea. International Congress on Mechanical Models in structural engineering.

[Rixen, 2001] Rixen, D. J. 2001. Generalized Mode Acceleration Methods and Modal Truncation Augmentation. Journal AIAA.

[Rixen, 2009] Rixen, D. J. 2009. Dual Craig-Bampton with Enrichment to Avoid Spurious ModesSociety for Experimental Mechanics Inc., for IMAC XXVII. 
[Roy \& Girard, 2005] Roy, N., \& Girard, A. 2005. Impact of Residual Modes in Structural Dynamics. Proceedings of the European Conference on Spacecrafts Structures, Materials and Mechanical Testing (ESA SP-581).

[Schawarz et al. , 2007] Schawarz, B., Richardson, M., \& Formenti, D. L. 2007. FEA Model Updating Using SDM. IMAC XXV.

[Schwarz \& Richardson, 1997] Schwarz, B. J., \& Richardson, M. H. 1997. Structural Modifications Using Higher Order Elements. IMAC XV.

[Sosnicki et al. , 2006] Sosnicki, O., Barillot, F., Leger, M., \& Claeyssen, F. 2006. Active Damping of Vibrations Applied on Ski Structures. International Conference on new actuator.

[Soulier et al. , 2003] Soulier, G., Gaechter, J., \& Dilhan, D. 2003. Very Low Shock Release Pyromechanisms. ESMATS Symposium. ESA SP-524.

[Sutter et al. , 1986] Sutter, T. R., Camarda, C. J., Walsh, J. L, \& Adelman, H. M. 1986. A Comparison of Several Methods for the Calculation of Vibration Mode Shape Derivatives. AIAA Journal, 256-265.

[Tham et al. , 2009] Tham, C., Christensen, E. R., Alldredge, D., Hahn, S., \& Howsman, T. 2009. The Use of Mode Truncation Augmentation in the Ares I Liftoff Analysis. AIAA.

[Tooren et al. , 2003] Tooren, M. Van, Rocca, G. La, Krakers, L., \& Beukers, A. 2003. Design and Technology in Aerospace. Parametric Modeling of Complex Structure Systems Including Active Components.

[Udwadia \& Esfandiari, 1990] Udwadia, F., \& Esfandiari, R. 1990. Nonclassicaly Damped Dynamic Systems: An Iterative Approach. Journal of Applied Dynamics, 57, 423-433.

[Vibrant, 2014] Vibrant. 2014. Using a Tuned Absorber to Suppress Vibration. Tech. rept. Application note 11. Vibrant Technology.

[Wallack et al. , 1989] Wallack, P., Skoog, P., \& M-Richardson. 1989. Comparison of Analytical and Experimental Rib Stiffener Modifications to a Structure. IMAC VII. 
[Wilson, 2000] Wilson, E. L. 2000. Three Dimensional Static and Dynamic Analysis of Structures. Computers and Structures, Inc.

[Wilson, 1985] Wilson, E.L. 1985. A New Method of Dynamic Analysis for Linear and Nonlinear Systems. Finite Elements for Analysis and Design, 21-23.

[Xie et al. , 2015] Xie, L., Baguet, S., Prabel, B., \& Dufour, R. 2015. Parametric Analysis of the Nonlinear Behavior of Rotating Structures. Journal of vibration and acoustics.

[Yang et al. , 2002] Yang, G., Spencer, B., \& Leban, F. 2002. Shock Isolation Using Smart Damping. Journal of structural control, 135-152.

[Zeng, 1995] Zeng, Q. 1995. Highly Accurate Modal Method for Calculating Eigenvector Derivatives in Viscous Damping Systems. AIAA, 33(4), 746-751. 